\title{
CALPHAD Based Cellular Model of Dendrite Growth in Welds
}

\author{
by
}

\section{Ghazi H. Alsoruji}

\begin{abstract}
A thesis submitted to the Faculty of Graduate and Postdoctoral Affairs
in partial fulfillment of the requirements for the degree of

Doctor of Philosophy

in

Mechanical Engineering

Department of Mechanical and Aerospace Engineering

Carleton University

Ottawa, Ontario
\end{abstract}

(C) 2020

Ghazi H. Alsoruji 


\section{Abstract}

A computational model of dendritic growth based on the CALPHAD (the CALculation of PHAse Diagrams) thermodynamic model was developed in this study. The dendrite growth was simulated using cellular automata (CA) with the equilibrium conditions in interfacial cells. The developed novel model (CA-CALPHAD) overcomes the current limitation of the published CA models, in which linearized phase diagrams are used, and allows for the investigation of some practical alloys such as stainless steels. To reduce the computational time, the study proposes a specific data structure to store the thermodynamic information and an efficient interpolation scheme to retrieve the information during the simulation.

The model takes into account the curvature effect of the evolving solid/liquid (S/L) interface by incorporating the capillarity undercooling into the thermodynamic information during the simulation. Two methods of calculating the $\mathrm{S} / \mathrm{L}$ interface curvature are investigated in the current study: the level-set and cell-count. It was found that the levelset method is more accurate and less sensitive to the mesh anisotropy. In addition, the levelset method was optimized to obtain the highest possible accuracy and to mitigate the effect of mesh anisotropy. It was demonstrated that the choice of different models for the curvature calculation could lead to significantly different simulation results, i.e., the dendrite morphology, segregation pattern, and grain size. The finite volume (FV) numerical scheme was used to solve the mass and heat diffusion equations. In addition, 
imposing a temperature profile with a constant cooling rate and temperature gradient instead of solving the heat equation was verified.

The developed CA-CALPHAD model can be used to investigate the free growth, constrained growth, and competitive growth of dendrites in response to different solidification parameters. The results of modeling include the dendrite morphology, dendrite size, solute segregation in the dendrite, dendrite growth rate, dendrite tip radius, and the spacing between primary and secondary dendritic arms. In addition, the model can be used to investigate the solidification of a duplex steel so that the fractions of ferrite and austenite can be estimated in the final microstructure. The investigations of a ternary stainless steel alloy (Fe-Cr-C) demonstrate that a higher cooling rate increases the solute segregation and the potential of sensitization, and produces a finer grain.

Finally, a procedure of linking the developed CA-CALPHAD model to a computational welding mechanics tool (CWM) was developed producing a holistic multiphysics model (CWM-CA-CALPHAD). Therefore, the model can be used to predict the microstructure of a weld in response to realistic welding parameters and weld joints design. 
I dedicate this work to my family. 


\section{Acknowledgments}

Primarily I would like to thank Allah for being able to complete this study. Then I would like to express my sincere gratitude to my supervisors, professor Andrei Artemev and professor John Goldak for their infinite support. I would also like to thank professor

Goldak's team for their corporation. Finally, I owe many thanks to Carleton University and the Department of Mechanical and Aerospace Engineering. 


\section{Table of Contents}

Abstract $\quad$ i

Acknowledgments $\quad$ iv

List of Figures $\quad$ ix

List of Symbols $\quad$ xvi

Chapter-1: Introduction 1

1.1 The Objective of the Study 1

1.2 Theses Content 2

1.3 Computational Welding Mechanics CWM 4

1.4 Welding Metallurgy

1.5 Fusion Zone Microstructure 9

1.5.1 The Morphology of the Solidification Microstructure 10

1.5.2 Weld Pool Microstructure 11

1.6 Welding Parameters 13

1.7 Solidification Microstructure 14

1.7.1 Solidification Driving Force 15

1.7.2 The Capillarity Effect 17

1.7.3 Analytical Dendrite Growth Models 18

1.7.4 Nucleation $\quad 21$

1.7.5 Surface Energy Anisotropy 23

1.8 Solidification Modeling 25

1.8.1 Macroscopic Solidification Modeling 28

1.8.2 Mesoscopic Solidification Modeling 30

1.8.3 Microscopic Solidification Modeling (Cellular Automata Modeling) 32

1.8.4 Nanoscopic Solidification Modeling (Phase Field Modeling) 33 
1.9 CALPHAD vs. The Linearization Approach of Phase Diagram 37

Chapter-2: The Computation of Phase Diagram (CALPHAD) 40

2.1 Thermodynamics Potentials $\quad 40$

2.2 Internal Energy (U) and Gibbs Energy (G) 41

2.3 Metallurgical Thermodynamics 43

2.4 General Thermodynamics Equilibrium 44

2.5 Phase Equilibria and Gibbs Energy $\quad 46$

2.5.1 Phase Stability of Metallic Alloys 47

2.6 Phases Stability and Chemical Potentials 50

2.7 Ternary Phase Diagram and Gibbs Energy 54

2.8 Thermodynamics Models for CALPHAD 57

2.8.1 Ideal Solution Model 58

2.8.2 Regular Solution Model $\quad 60$

2.8.3 Real Solution Model $\quad 62$

2.8.4 Sublattice Thermodynamics Model 64

2.8.4.1 An Example of a Sublattice Model 64

2.8.4.2 The Gibbs Energy in the Sublattice Model 66

2.9 Phase Diagram as an Optimization Problem 66

2.9.1 Global Optimization Formulation of Gibbs Energy Minimization 67

2.9.2 Phase Diagram from Chemical Potentials Equalities 69

2.10 Fe-Cr-C Thermodynamics Modeling 71

2.11 The Implementation of Fe-Cr-C Thermodynamics Model 75

2.12 Calculation Algorithm and Initial Guess 79

2.13 The Validation of The CALPHAD Solver 81

Chapter-3: Solidification Modeling by Cellular Automata CA 84

3.1 Cellular Automata (CA) 84 
3.1.1 Cellular Automata Computational Domain and Cell Neighborhood Definition 85

3.1.2 Cellular Automata Transition Rules and Mesh Anisotropy 87

3.1.3 Cellular Automata State Variable Calculations 89

3.1.4 The Computation of S/L Interface Curvature 92

3.2 Overview of Some Cellular Automata Models 95

$\begin{array}{ll}3.3 \text { Computational Tools } & 97\end{array}$

3.3.1 Mass Transport Computation Tool 98

3.3.2 Imposed Temperature Field Computation 102

3.3.3 Heat Transport Computation 102

$\begin{array}{ll}\text { 3.3.4 Curvature Computation } & 104\end{array}$

3.3.4.1 Curvature Computation by Cell Count Algorithm 104

3.3.4.2 Curvature Computation by the Level Set Method 105

$\begin{array}{ll}\text { 3.3.5 The Sphere Test } & 107\end{array}$

3.3.5 Solid Fraction Computation Tool 110

3.4 Coupling CALPHAD Data to Cellular Automata Modeling 113

3.5 Obtaining Information from the CALPHAD Data 115

3.5.2 Pointwise Interpolation Procedure for Reading Solid Fraction 119

3.5.3 Bezier Curves Representation of Phase Diagrams 120

3.6 Alloy Properties and Simulation Parameters 121

3.7 Computational Domain (CD) Initialization 122

3.8 The Algorithm of the Simulation $\quad 124$

3.9 Model Verification and Validation $\quad 126$

3.9.1 Mass Conservation $\quad 126$

3.9.2 Simulated Dendritic Structures 127

3.9.3 The Selection of Cell Size 129

Chapter-4: CALPHAD-Cellular Automata Coupling Results 132 
4.1 The Effect of Surface Energy Anisotropy on the Free Growth of Equiaxed Grain 134

4.2 The Effect of Undercooling on the Free Growth of Equiaxed Grain

4.3 The effect of cooling rate

4.4 Solidification of Duplex Steels

4.5 The Effect of Curvature Calculation Models on a Microstructure Evolution

4.6 Dendrite Growth Simulations Including Heat Diffusion Solver

Chapter-5: Predicting the Microstructure of the Fusion Zone

5.1 Coupling Macroscopic Welding Case to Microscopic Solidification Model

5.2 The Microstructure Evolution of the Weld Pool Boundary

5.3 The Microstructure Evolution with a Duplex Structure

Chapter-6: Conclusions

Contribution to Knowledge

Future Work

List of References 


\section{List of Figures}

Figure-1.1: Physical phenomena of the fusion welding process. 6

Figure-1.2: The microstructure of a weld joint of $\mathrm{Fe}-\mathrm{C}$ alloy in response to a thermal cycle. [7], page-102.

Figure-1.3: Grains competitive growth. [9], page-176.

Figure-1.4: The microstructure-process parameters map as predicted by the constitutional supercooling theory. [9], page-166.

Figure-1.5: The variation of solidification parameters according to the shape of the weld pool. Figure-b is from [9], page-202.

Figure-1.6: (a) The main microstructure features, displaying the model of the dendrite envelope as an ellipsoid. [11], page-323. (b) the size of equiaxed grain.

Figure-1.7: The components of undercooling.

Figure-1.8: The effect of curvature on the melting temperature. $T^{e q}$ is the equilibrium melting temperature of a flat interface, $T^{\text {convex }}$ is the melting temperature of a convex interface, and $T^{\text {concave }}$ is the melting temperature of a concaved interface.

Figure-1.9: (a) hemispherical model and (b) paraboloid of revolution model.

Figure-1.10: Critical size for nucleation.

Figure-1.11: Crystal growth with (a) anisotropic Surface energy (dendrite), and (b) isotropic Surface energy (coral-like). [11], page-317.

Figure-1.12: The angles that describe the anisotropy in 3D. 25

Figure-1.13: Solidification modeling length scales. [12], page-2. 27

Figure-1.14: (a) enthalpy function for pure materials, (b) alloys, (c) real alloys. [13]

Figure-1.15: Grain envelope geometry used in the mesoscale solidification modeling. 
Figure-1.16: An experimental and mesoscale CA simulated macrostructures of an $\mathrm{Al}-4.5 \% \mathrm{Cu}$ alloy in casting with various pouring temperatures: (a) $710{ }^{\circ} \mathrm{C}, \quad 31$ (b) $760^{\circ} \mathrm{C}$ and (c) $810^{\circ} \mathrm{C}$. [13], page- 231 .

Figure-1.17: (a) continuous interface, (b) sharp interface.

Figure-1.18: Linearization approach of (a) Al-Cu binary system and (b) Al-Si binary system. [30], pages-286, 316 .

Figure-1.19: Fe-Cr binary phase diagram.

Figure-2.1: The variation of Gibbs free energy with the arrangement of atoms. 47

Figure-2.2: Gibbs energy and the stability of a binary system. [35], page-33. 49

Figure-2.3: The Gibbs energy curves at eutectic reaction. [35], page-37. 49

Figure-2.4: Chemical potentials at constant temperature and pressure for (a) non-equilibrium state and (b) Equilibrium state.

Figure-2.5: The graphical interpretation of chemical potentials in a binary system.

Figure-2.6: The common tangent line and stability.

Figure-2.7: Phase stability with two common tangent lines. 53

Figure-2.8: The construction of a ternary phase diagram. 55

Figure-2.9: Reading equilibrium information from isotherms with (a) tie lines and (b) tie triangles.

Figure-2.10: The terms in Equation-2.23 for Al-Mg alloy.

Figure-2.11: The effect of $\Delta H_{\text {mix }}$ on the $\Delta G_{\text {mix }}$ at different temperatures $T$. [35], page-21.

Figure-2.12: An example of a sublattice model. 65

Figure-2.13: The total Gibbs energy of two phases in contact. 69

Figure-2.14: A comparesion between Andersson and Alexandra's thermodynamic assessments.

Figure-2.15: The mushy zone of L-BCC as calculated by the L-BCC solver. $\quad 78$

Figure-2.16: Different mushy zones in temperature range two. 78 
Figure-2.17: The algorithm of the solution. $\quad 79$

Figure-2.18: The verification of the triangle zone. 81

Figure-2.19: The isotherm on the left was calculated at $\mathrm{T}=1437^{\circ} \mathrm{C}$ by ThermoCalc $\AA$, and (b) the isotherm on the right was calculated by the developed model.

Figure-2.20: (a) The isotherm at $\mathrm{T}=1500^{\circ} \mathrm{C}$ calculated by Thermo-Calc $\AA$ and (b) the isotherm calculated by the developed model.

Figure-3.1: The definition of neighborhood of an interfacial cell. [47], page217.

Figure-3.2: Geometrical assumptions of the moving S/L interface inside a cell. $\quad 90$

Figure-3.3: (a) a dendrite with actual curvature, (b) the dendrite with overestimated curvature, and (c) the dendrite with underestimated curvature.

Figure-3.4: Calculating curvatures by cell count method. 93

Figure-3.5: Finite difference formulation of an interfacial cell. 99

Figure-3.6: The computational domain of mass diffusion computation. 101

Figure-3.7: Imposed temperature profile. 102

Figure-3.8: The neighbor cells definition for cell count method. The curvature is evaluated for the middle cell $\left(\right.$ cell $\left._{i j k}\right)$.

Figure-3.9: The neighbor weights for a centered cell Cell $_{i j k}$.

Figure-3.10: The estimation of solid fraction for the sphere test.

Figure-3.11: The error of curvature estimation by different curvature calculation methods.

Figure-3.12: Pseudo binary liquidus and solidus lines to illustrate OCSM.

Figure-3.13: Incorporation the effect of capillarity undercooling for reading from CALPHAD data.

Figure-3.14: Thermodynamics lookup table.

Figure-3.15: The interaction between the solidification and CALPHAD models.

Figure-3.16: Three different thermodynamic states: liquid (qP1), solid (qP3), and mushy (qP2). 
Figure-3.17: The procedure of calculating the information of a query point ( $\mathrm{qP}$ ) in the mushy phase.

Figure-3.18: The inQuad locating procedure. (a) the quadrilateral shape area $=\mathrm{TA} 1+\mathrm{TA} 2$, (b) $\mathrm{qP}$ is inside the shape if $\mathrm{A} 1+\mathrm{A} 2+\mathrm{A} 3+\mathrm{A} 4<=$ quad area, (c) $\mathrm{qP}$ is outside the shape if $\mathrm{A} 1+\mathrm{A} 2+\mathrm{A} 3+\mathrm{A} 4>$ quad area.

Figure-3.19: Interpolate data between two different temperature values.

Figure-3.20: Data points that store thermodynamic information at $\mathrm{T}=1500{ }^{\circ} \mathrm{C}$.

Figure-3.21: The inverse weighted distance scheme.

Figure-3.22: Representing the solidus and liquidus of Al-Mg by Bezier curve.

Figure-3.23: Reading the solid fractions from a Bezier curve. (a) liquid state, (b) mushy state, and (c) solid state.

Figure-3.24: The initialization of a computational domain.

Figure-3.25: Three different initial solid/liquid interface for simulations.

Figure-3.26: (a) undercooled and (b) non-undercooled initial alloy composition.

Figure-3.27: The resulting dendrite tip.

Figure-3.28: (a) a scanning-electron micrograph of dendrites in a weld [64], and (b) the solidified microstructure of Fe-C-Cr [the developed model].

Figure-3.29: (a) and (b) show equiaxed dendrites with low anisotropy, with (a) from [65] and (b) from the developed CA-CALPHAD model. (c) and (d) show equiaxed dendrites with high anisotropy, with (c) from [65] and (d) from the developed CA-CALPHAD model.

Figure-3.30: The relationship between the tip radius (TipR) and the cell size $(\mathrm{dx})$.

Figure-3.31: The developed dendrite with cell size equal to (a) $0.05 \mu \mathrm{m}$, (b) $0.25 \mu \mathrm{m}$, and (c) $0.5 \mu \mathrm{m}$.

Figure-4.1: Unconstrained dendrite growth with very low anisotropy coefficients (anis=0.02). (a) initial solid fraction, (b) solid fraction at $\mathrm{t}=0.0005$ $\mathrm{sec}$, (c) solid fraction at $\mathrm{t}=0.00175 \mathrm{sec}$, (d) solid fraction at $\mathrm{t}=0.0025 \mathrm{sec}$. [RUN411] 
Figure-4.2: (a) the solute (carbon) distribution in liquid at $t=0.0005 \mathrm{sec}$, (b) the solute (carbon) distribution in liquid at $\mathrm{t}=0.0025 \mathrm{sec}$.

Figure-4.3: (a) the initial grain nucleus, (b) the grain at $\mathrm{t}=0.00039 \mathrm{sec}$, (c) the grain at $\mathrm{t}=0.0027 \mathrm{sec},(\mathrm{d})$ the grain at $\mathrm{t}=0.0093 \mathrm{sec}$. [RUN412]

Figure-4.4: (a) the chromium distribution in liquid, and (b) the carbon distribution in liquid. [RUN412]

Figure-4.5: The solute layer rejected in liquid as obtained by a) [66], b) [67], and c) [65].

Figure-4.6: A cross section of the dendrite showing (a) the curvature of the S/L interface, and (b) the capillarity undercooling. [RUN412]

Figure-4.7: (a) the initial grain nucleus, (b) the grain at $t=0.00039 \mathrm{sec}$, (c) the grain at $\mathrm{t}=0.0039 \mathrm{sec},(\mathrm{d})$ the grain at $\mathrm{t}=0.0079 \mathrm{sec}$. [RUN413]

Figure-4.8: (a) a cross section of the solid fraction at $t=0.0079 \mathrm{sec}$, and (b) the carbon distribution in solid. [RUN413]

Figure-4.9: (a) the developed dendrite of RUN421, and (b) the developed dendrite of RUN422.

Figure-4.10: (a) a cross section of the developed microstructure of RUN421 (low undercooling), and (b) a cross section of the developed microstructure of RUN422 (high undercooling).

Figure-4.11: The microstructure morphology of (a) RUN431, (b) RUN432, (c) RUN433, and (d) RUN434.

Figure-4.12: A simplifying assumption for isotherms.

Figure-4.13: For (Cr 8\%wt - C 0.5\%wt) alloy, the thermodynamics state is (a) L-BCC, and (b) L-FCC.

Figure-4.14: The duplex microstructure cooling rate $=10{ }^{\circ} \mathrm{C} / \mathrm{s}$. [RUN441]

Figure-4.15: The duplex microstructure cooling rate $=100{ }^{\circ} \mathrm{C} / \mathrm{s}$. [RUN442]

Figure-4.16: The carbon distribution in solid for (a) $10{ }^{\circ} \mathrm{C} / \mathrm{s}$ cooling rate [RUN441] and (b) $100^{\circ} \mathrm{C} / \mathrm{s}$ cooling rate [RUN442].

Figure-4.17: The initial S/L interface for RUN451, RUN452, RUN453, and RUN454. 
Figure-4.18: (a) the developed microstructure of RUN451 that uses cell-count method, and (b) a cross section of the solid fraction through x-axis. [RUN451]

Figure-4.19: The developed microstructure of RUN452 that uses level-set method, and (b) a cross section of the solid fraction through x-axis. [RUN452]

Figure-4.20: The disturbance of the initial $\mathrm{S} / \mathrm{L}$ interface due to cell-count method at (a) $0.0011 \mathrm{sec}$, (b) $0.0022 \mathrm{sec}$, (c) $0.0033 \mathrm{sec}$, and (d) $0.0045 \mathrm{sec}$.

Figure-4.21: The developed microstructure of RUN453 that uses level-set method with optimum averaging, and (b) a cross section of the solid fraction through x-axis. [RUN453]

Figure-4.22: The developed microstructure of RUN454 that uses level-set method with optimum averaging and $\Gamma=0.5^{-7} \mathrm{~K} \cdot \mathrm{m}$, and (b) a cross section of the solid fraction through x-axis. [RUN454]

Figure-4.23: (a) the initial S/L interface of RUN461, and (b) the initial $\mathrm{S} / \mathrm{L}$ interface of RUN462.

Figure-4.24: For RUN461 at $t=0.0078 \mathrm{sec}$, (a) the growth dendrite, (b) the rejected heat, (c) the rejected solute (carbon), and (d) the capillarity undercooling at a cross section.

Figure-4.25: For RUN461 at $\mathrm{t}=0.019 \mathrm{sec}$, (a) the growth dendrite, (b) the rejected heat, (c) the rejected solute (carbon), and (d) the capillarity 159 undercooling at a cross section.

Figure-4.26: For RUN461 at $\mathrm{t}=0.027 \mathrm{sec}$, (a) the growth dendrite, (b) the rejected heat, (c) the rejected solute (carbon), and (d) the capillarity 160 undercooling at a cross section.

Figure-4.27: The results of RUN462 at $\mathrm{t}=0.03$ sec. (a) the developed microstructure, (b) a cross section of the solid fraction field, (c) the temperature distribution, (4) the rejected solute.

Figure-4.28: The developed microstructure at (a) $\mathrm{T}=0.0078 \mathrm{sec}$, (b) $\mathrm{T}=0.0156$ sec, (c) $\mathrm{T}=0.0234 \mathrm{sec}$, and (d) $\mathrm{T}=0.0234 \mathrm{sec}$. [RUN462]

Figure-4.29: The temperature profile at (a) 1000 time step $(0.0039 \mathrm{sec})$, (b) 2000 time step $(0.0078 \mathrm{sec})$, (c) 5000 time step $(0.019 \mathrm{sec})$, and (d) 8000 time step $(0.031 \mathrm{sec})$. 
Figure-5.1: The used welding joint for macroscopic temperature calculation. $\quad 167$

Figure-5.2: (a) the cooling curve at $\mathrm{P}_{0}$, (b) the cooling rate at $\mathrm{P}_{0} .168$

Figure-5.3: The variations of cooling rate and temperature gradient around the weld pool. [9], page-201.

Figure-5.4: (a) the developed microstructure of $\mathrm{P}_{0}($ RUN511) at $\mathrm{t}=0.01 \mathrm{sec}$, and (b) the resulting temperature gradient in ${ }^{\circ} \mathrm{C}$. [RUN511]

Figure-5.5: (a) the distribution of carbon in solid, and (b) the $\mathrm{C} / \mathrm{Cr}$ ratio. [RUN511]

Figure-5.6: (a) the microstructure of $\mathrm{P}_{2}(\mathrm{RUN512})$ at $\mathrm{t}=0.01 \mathrm{sec}$, and (b) the resulting temperature gradient in ${ }^{\circ} \mathrm{C}$. [RUN512]

Figure-5.7: (a) the distribution of carbon in solid, and (b) the $\mathrm{C} / \mathrm{Cr}$ ratio. [RUN512].

Figure-5.8: (a) the microstructure of RUN513 at $\mathrm{t}=0.011 \mathrm{sec}$, and (b) the carbon distribution in liquid. [RUN513]

Figure-5.9: (a) the distribution of carbon in solid, and (b) the $\mathrm{C} / \mathrm{Cr}$ ratio. [RUN513].

Figure-5.10: (a) the microstructure of RUN514 at $\mathrm{t}=0.012 \mathrm{sec}$, and (b) the carbon distribution in liquid. [RUN514]

Figure-5.11: (a) the distribution of carbon in solid, and (b) the $\mathrm{C} / \mathrm{Cr}$ ratio. [RUN514]

Figure-5.12: (a) the microstructure of RUN521, and (b) a cross section through the x-axis. [RUN521]

Figure-5.13: (a) the produced ferrite phase, (b) the produced austenite phase, and (c) the developed duplex microstructure. [RUN521] 


\section{List of Symbols}

$V \quad$ Dendrite growth rate.

TG Temperature gradient.

Rc Cooling rate.

S/L Solid-liquid interface.

$D_{L} \quad$ Diffusion coefficient of solute atoms in liquid

$\Delta T_{0} \quad$ Alloy freezing range.

FZ Fusion zone.

HAZ Heat affected zone.

$\lambda_{1} \quad$ Dendrite primary dendrite spacing.

$\lambda_{2} \quad$ Dendrite secondary arm spacing.

$\Delta T \quad$ Total undercooling.

$\Delta T_{t h} \quad$ Thermal undercooling

$\Delta T_{c} \quad$ Solutal undercooling or constitutional undercooling.

$\Delta T_{r} \quad$ Capillarity undercooling

$\Delta T_{k} \quad$ Kinetic undercooling

$T_{m}^{e} \quad$ Equilibrium melting temperature.

$C_{o} \quad$ Nominal or initial alloy composition.

$C_{C r}^{l} \quad$ The equilibrium weight fraction of chromium in liquid.

$C_{C}^{l} \quad$ The equilibrium weight fraction of carbon in liquid.

$C_{C r}^{S} \quad$ The equilibrium weight fraction of chromium in solid.

$C_{C}^{S} \quad$ The equilibrium weight fraction of carbon in solid.

$\Gamma \quad$ Gibb-Thomson coefficient

$\mathrm{Kr} \quad$ Solid-liquid interface curvature.

G Gibbs energy.

$P_{C} \quad$ Solutal Peclet number

$P_{T} \quad$ Thermal Peclet number 
$\Omega_{C} \quad$ Solutal dimensionless supersaturation

$C^{l} \quad$ Solute concentration in liquid.

$C^{s} \quad$ Solute concentration in solid.

Lh Latent heat of fusion.

$C p \quad$ Heat capacity.

$R_{\text {tip }} \quad$ Dendrite tip radius.

$\gamma \quad$ Surface energy.

$\gamma(\bar{n}) \quad$ Anisotropy function of surface energy.

$\bar{n} \quad$ The normal vector of any point on the Solid-liquid surface.

$\varepsilon \quad$ Anisotropy coefficient.

$\rho \quad$ Density.

$k_{t h} \quad$ Thermal conductivity.

$H \quad$ Enthalpy.

$f^{s} \quad$ Solid fraction.

$T^{l} \quad$ Liquidus temperature.

$T^{S} \quad$ Solidus temperature.

$\dot{n} \quad$ Grain nucleation rate.

$k_{o} \quad$ Alloy distribution coefficient.

$\mu_{k} \quad$ Kinetic coefficient.

$\varnothing \quad$ Phase-field order parameter.

$f \quad$ Free energy density function. 


\section{Chapter-1: Introduction}

\subsection{The Objective of the Study}

The main contribution of this study is coupling CALPHAD (the CALculation of PHAse Diagrams) with a Cellular Automata (CA) based solidification modeling. The developed model is integrated into $\operatorname{VrWeld}{ }^{\circledR}$ (a FE software developed by Goldaktec $($ ) ) to predict weld microstructure responses to specific weld parameters and weld joint designs. Solidification modeling of an alloy involves thermodynamics properties, specifically the relationship between equilibrium temperature and composition. The common approach is to assume a linear relationship between the two. This assumption excludes using the model for a wide range of practical alloys and superalloys. The main advantages of CAPHAD are to increase the ability of the CA solidification model to study any alloy. In addition, model uncertainty will be reduced, since the relationship between equilibrium temperature and composition is calculated rather than approximated.

The current solidification model is based on many previous models, the first of which was proposed by Artemev and Goldak [1]. They studied the dendritic growth of an Al-Si binary system in two dimensions. They assumed a constant undercooling temperature and verified the results by comparing the computed growth velocity and tip radius with an analytical model. In [2], Martinez and Artemev extended the model to study a ternary alloy, Al-Si- 
$\mathrm{Cu}$, in 3D at a constant undercooling temperature. In [3], Ghazi and Artemev developed the model to include a transient temperature field, by imposing a temperature profile and cooling rate. They also investigated the effect of the initial solid/liquid interface morphology on the evolved microstructure, and the results were compatible with constitutional undercooling theory. The model was further developed by Komeil, Goldak, and Artemev to allow coupling of the solidification model with a finite element heat transient solution of the welding process [4]. In addition, they used an implicit solver to solve the diffusion equation of solutes.

All of the mentioned models were based on the kinetics of the solid/liquid $(\mathrm{S} / \mathrm{L})$ interface, and the movement of the interface was calculated at each time step. If the kinetics is underestimated, the microstructure evolves too slowly taking more computational time. On the other hand, the solution becomes unstable if the kinetics is overestimated. Computationally, the calculation of the kinetics imposes some constrains on the time step. In contrast, coupling CA with CALPHAD (i.e. CA-CALPHAD) does not require interface kinetic calculation that imposes less restrictions on the time step, and the mass balance is perfectly conserved.

\subsection{Theses Content}

The first chapter introduces into the research topic and summarizes the objectives. The state of the art is described in the field of solidification theory, welding metallurgy, morphology of the weld pool, the effects of welding parameters (i.e. welding velocity and 
welding power) on the solidification microstructure, and, finally, multiscale algorithms for solidification modeling with emphasis on microscale modeling. The key topic of first chapter is to show the importance of using CALPHAD calculation in solidification modeling to overcome the limitation of phase diagrams linearization approach.

Chapter-2 describes the metallurgical thermodynamics, since this is the theoretical background of CALPHAD method. It explains the fundamentals of phases stability using Gibbs energies and chemical potentials and the construction of phase diagrams. The chapter explains CALPHAD models such as ideal solutions, regular solutions, real solutions using Redlich-Kister polynomial approximation, and the compound energy model with sublattices. In addition, the chapter describes the implementation of the thermodynamic optimization code for $\mathrm{Fe}-\mathrm{Cr}-\mathrm{C}$ system. The chapter also outlines the equations that are written down to solve the equilibrium problem for the different cases of stable phases in this ternary system. The solution procedure is solved using MatLab functions. Finally, the predictions of the implemented Fe-Cr-C model are compared to

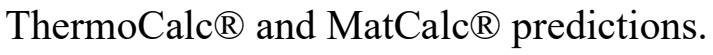

The following chapter 3 is related to Cellular Automata (CA) modeling which is subdivided into two parts. The first part presents an overview of existing techniques and CA models in relation to solidification modeling. In addition, the effect of physical and mesh anisotropy on microstructure morphology is explained, as is the impact of the $\mathrm{S} / \mathrm{L}$ interface curvature on the microstructure growth using kinetics and thermodynamics approaches. 
The second part of the chapter describes the details of the developed CA-CALPHAD model. The treatment starts with the computational tool for mass transport, i.e., diffusion of atoms through the simulation domain, then the temperature field calculation, curvature computation and solid fraction determination. In addition, the chapter describes the procedure to pre-evaluate the thermodynamic data of the system and then perform a table look-up to interpolate the values for the actual computation without necessity to compute thermodynamics in every finite volume.

Chapter-4 is focused on the result obtained with the present CA-CALPHAD model. First, the effect of surface energy anisotropy, undercooling degree, and cooling rate on a dendrite free growth are investigated. In addition, the solidification of a duplex microstructure with different cooling rate is investigated.

In the next chapter 5, the present model is applied to welding processes. The first section describes the evaluation of solidification parameters at specific points along the weld pool perimeter of a Bead-On-Plate weld joint. These data are subsequently used as input parameters for the microstructure simulation with CA-CALPHAD model. The dendrite morphology and chemical composition are analyzed. Finally, the conclusions are summarized and the contribution to knowledge is stated in Chapter-6.

\subsection{Computational Welding Mechanics CWM}

This study is intended to extend the field of computational welding mechanics (CWM) by incorporating thermodynamically accurate microstructure modeling. CWM is a tool that 
applies numerical methods (i.e. finite element FE and finite difference FD) to welding aid design, and can be used to calculate of important characteristics such as temperature distributions, distortions (i.e. shrinkage and buckling), thermal and residual stresses, and metallurgical changes due to the thermal cycle of the welding process. One might ask: why do we need CWM when professionally-verified welding design codes are available? This question can be addressed by considering three factors. The first is related to the conservative nature of design codes that produces conservative designs, which are costly and can limit the design due to geometrical considerations. The second factor is that most manufacturers must conduct trial-and-error experiments to build their in-house experience. CWM tools can reduce the number of such experiments. And the final factor is that new materials are frequently introduced into the industry and not included in the current design codes, which means that more trial-and-error experiments are required.

Using CWM can reduce the cost of welding design by minimizing the required number of trial-and-error experiments by up to fifty percent [5]. In addition, CWM can enhance our understanding of the welding process itself. For example, in [6] Goldak et al used the CWM tool, $\mathrm{VrWeld}{ }^{\circledR}$, to compute the transient temperature and evolution of the microstructure in a bead-on-plate weld. They demonstrated that characterizing welds only by power per unit length, as most researchers do, is not sufficient to specify a unique design. By using CWM, they demonstrated that welds should be characterized by either weld speed or weld power, as well as the power per unit length. The long-term objective of CWM is to predict the quality, reliability, properties, and manufacturing cost of welds and welded structures. 
Achieving the long-term objective requires a holistic simulation of all relevant welding phenomena, from the arc to the micro and macroscopic processes of the welded products. The simulation is inherently complex because it involves multi-physics and coupled phenomena. Figure-1.1 illustrates how three main groups of processes interact in a welding simulation.

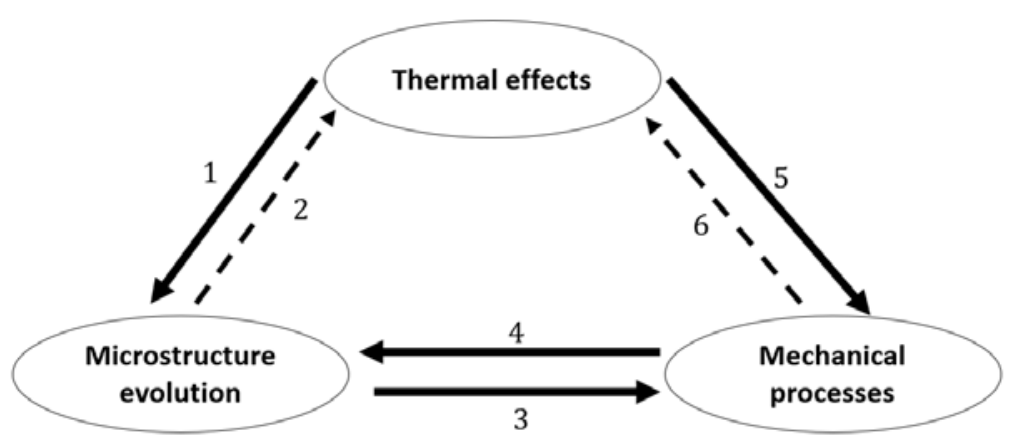

Figure-1.1: Physical phenomena of the fusion welding process.

Thermal analysis is at the top of the diagram because all aspects of welding simulation are driven by the transient temperature field. The bold arrows indicate strong coupling between processes, and the dashed arrows signify weak coupling. The thermal field during welding causes mechanical deformation and thermal stress, as indecated by the fifth arrow, and microstructural changes, as shown by the first arrow. Phase transformation processes release or absorb latent heat, which affects the thermal field by acting as a heat sink when heating and a heat source when cooling, as depicted by the second arrow. In addition, phase transformation processes cause volume changes which affect plastic and elastic material behavior, as shown by the third arrow. The sixth arrow shows the minor effect of mechanical elastic-plastic deformation on thermal analysis. 


\subsection{Welding Metallurgy}

A weld joint microstructure evolution is driven primarily by the thermal distribution created by the welding arc. Once the arc is applied the heat transfers to the weld joint and then diffuses into the weld joint and the material around it. The distribution of heat and temperature is mainly determined by the welding parameters, the weld joint material, the weld joint thickness and the design. For example, as the thermal conductivity of aluminum is high, heat diffuses rapidly in aluminum weld joints, corner fillet weld joints diffuse more heat than ordinary fillet joints, and thick weld joints can absorb more heat than thinner joints.

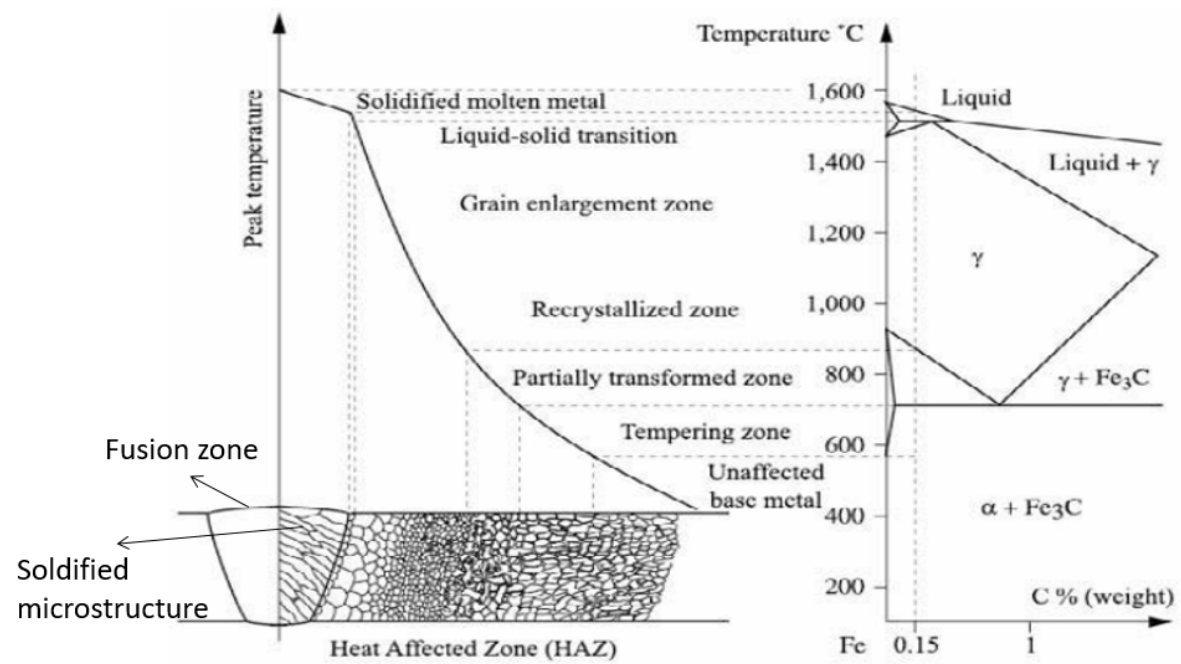

Figure-1.2: The microstructure of a weld joint of $\mathrm{Fe}-\mathrm{C}$ alloy in response to a thermal cycle. [7], page-102.

The distribution of temperature in a weld joint forms two distinct zones: the fusion zone (FZ) and the heat-affected zone (HAZ), while the rest of the weld joint microstructure is not significantly affected by the welding process. Each zone has different thermal cycles, 
peak temperatures, and cooling rates. Thus, the evolutions of microstructure and composition are different in FZ and HAZ. In HAZ, the base material endures solid-state transformations, grain growth, and grain refinement, as shown in Figure-1.2. The temperature is high enough in the FZ to melt the material, and the molten material solidifies as the FZ cools down. Due to the solidification process, the FZ acquires an entirely different microstructure than the base material and different composition if filler metal is used.

The quality of a weld is determined by its mechanical properties which in turn depend on the weld microstructure. For example, the grain size strongly affects the mechanical strength and fatigue resistance, as small grains have more grain boundary area that impedes the dislocation movement and increases the low temperature strength. Similarly, small grains increase the fracture toughness and, consequently, fatigue life. Thus, grain refinement is the only strengthening mechanism that increases both the strength and the fracture toughness of alloys [8]. During welding, grain size can be tailored by controlling the solidification process and adjusting the welding parameters (i.e. welding speed and welding power). As well as grain size, grain composition also determines weld quality. Ideally, the composition should be homogenous to ensure the weld also has homogenous properties. Solidification processes can be controlled to create microstructures that are as highly homogenous as possible. Non-homogenous composition can produce a cored microstructure in which alloying components with low melting temperatures segregate at the grain boundaries, and this segregation can cause hot cracks and in-service cracks. The prediction of microstructure responses to welding parameters requires a holistic 
multiphysics model that links welding parameters with microstructure evolution. This can be achieved by CWM and is the main goal of this study.

\subsection{Fusion Zone Microstructure}

The microstructure of the fusion zone in a weld is a solidified microstructure in which columnar grains, dendritic grains, and in some cases equiaxed grains are formed. The nucleation of a solid phase in the weld is mainly heterogeneous, which has a low energy barrier due to the presence of an existing solid interface at a fusion boundary. However, equiaxed grains can form with high undercoolings. The growth of grains (away from the fusion boundary) is governed by a competitive growth mechanism, as shown in Figure-1.3. With competitive growth, the dendrites that grow quickly will impede the growth of others. If a dendrite is growing in its easy growth direction (i.e. $<100>$ for both FCC and BCC materials) and/or in the direction of the maximum temperature gradient, the growth will be relatively fast [9]. The final microstructure of a weld is typically composed of many columnar/dendritic grains in the fusion zone [10].

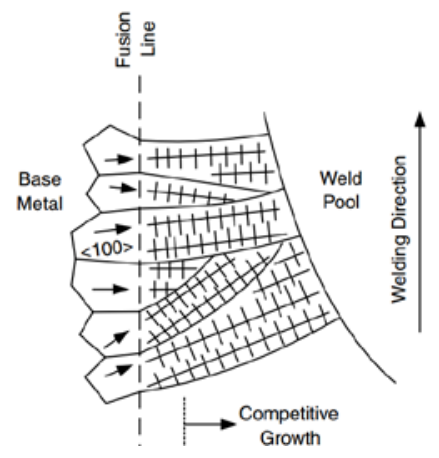

Figure-1.3: Grains competitive growth. [9], page-176. 
The resulting microstructure of welds always has nonhomogeneous compositions. Microsegregation is a chemical inhomogeneity that decreases weld quality by causing hot cracking and cold cracking. It occurs due to the redistribution of atoms during the solidification process, which depends on both thermodynamics and kinetics, or thermal fluctuations during the welding process, known as banding. Solidification parameters, growth rate $(V)$ and temperature gradient $\left(T_{G}\right)$, as well as the phase diagram of a material, determine both the microsegregation and the morphology of a solidified structure.

\subsubsection{The Morphology of the Solidification Microstructure}

The shape of a $\mathrm{S} / \mathrm{L}$ interface during the solidification of a melt determines the morphology of the final microstructure. Typically, an S/L interface can be planar, columnar, dendritic, equiaxed, or a combination of these. In the case of welding, the mode of solidification is usually cellular, dendritic or a combination of the two, depending on the solidification parameters [10]. Chalmer et al [9] proposed the constitutional supercooling theory, an experimentally validated theory, that describes the effect of solidification parameters on microstructure morphology. The theory mathematically defines when a planar S/L interface becomes unstable and develops a more complex morphology, and it also qualitatively describes when the solid phase morphology is cellular, dendritic, or equiaxed. Essentially, the constitutional supercooling theory proposes that the S/L interface could be flat if the initial solute concentration of the alloy and the growth rate are small and the thermal gradient is high. Mathematically, constitutional supercooling proposes that the $S / L$ 
interface will be flat if $\frac{T_{G}}{V} \geq \frac{\Delta T_{0}}{D_{L}}$ [9], where $T_{G}$ is the thermal gradient, $V$ is the growth rate, $\Delta T_{0}$ is the equilibrium freezing range, and $D_{L}$ is the diffusion coefficient of solute atoms in liquid. The main predictions of the constitutional supercooling theory are that $T_{G} / V$ determines the morphology of the solidification structure, and $T_{G} \times V$ (i.e. cooling rate) determines grain size, as shown in Figure-1.4.

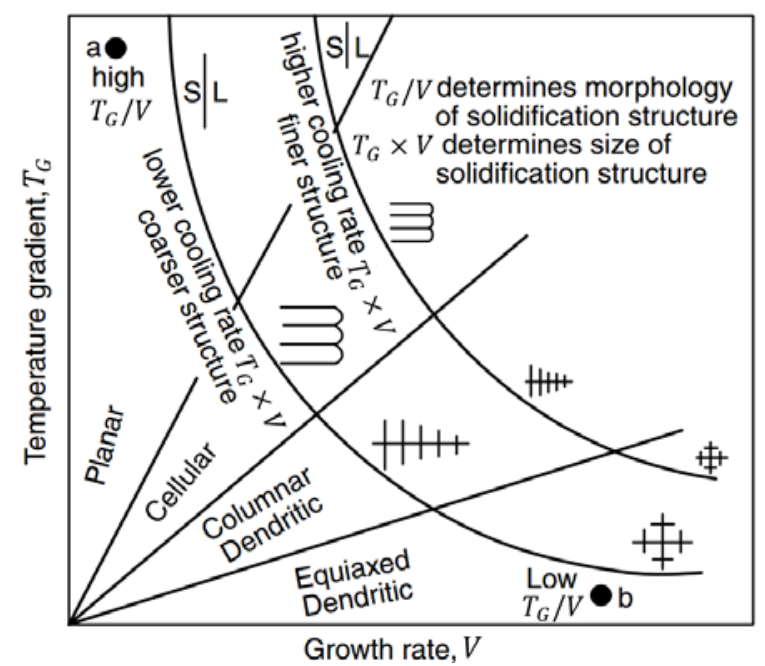

Figure-1.4: The microstructure-process parameters map as predicted by the constitutional supercooling theory. [9], page-166.

\subsubsection{Weld Pool Microstructure}

Solidification parameters vary in a single weld pool from the weld fusion line boundary (FL) to the weld fusion centerline (CL), as shown in Figure-1.5. These variations, and the shape of the weld pool, determine the macrostructure of the weld fusion zone. Figure-1.5a indicates how solidification parameters vary around a weld pool, and Figure-1.5b depicts the resulting microstructure. 


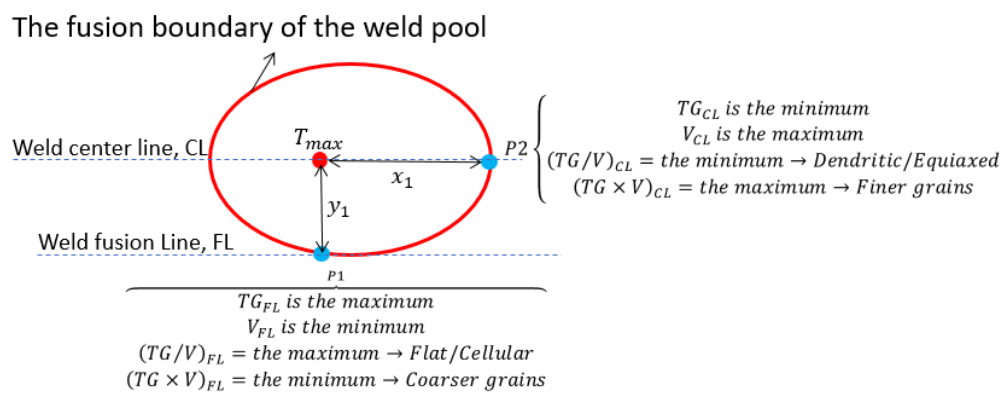

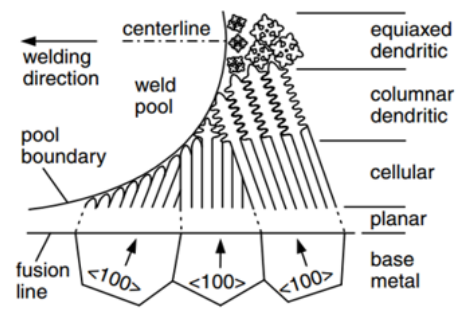

(b)

Figure-1.5: The variation of solidification parameters according to the shape of the weld pool. Figure-b is from [9], page-202.

According to the presented description in [9] and illustrated in Figure-1.5a, the temperature gradient at point $\mathrm{P} 1$ is maximum, since the distance $y_{1}$ is the shortest distance between the source of heat and the fusion line boundary. Since the thermal gradient is the steepest along $y_{1}$, the growth rate is minimum at $\mathrm{P} 1$. In contrast, the temperature gradient at point $\mathrm{P} 2$ is minimum since the distance $x_{1}$ is the longest, and the growth rate is the maximum. Therefore, the ratio of $T G / V$ at $\mathrm{P} 2$ is the minimum, and the ratio $T G / V$ at $\mathrm{P} 1$ is the maximum. Mathematically, $(T G / V)_{C L} \ll(T G / V)_{F L}$ which suggests that the solidification mode at P2 could be dendritic or equiaxed, and the solidification mode at P1 could be cellular or flat, according to the constitutional supercooling theory. Moreover, the ratio $(T G \times V)$ at $\mathrm{P} 2$ is the maximum since it is located where the cooling rate is maximum, and the ratio $(T G \times V)$ at $\mathrm{P} 1$ is the minimum. Mathematically, $(T G \times V)_{C L}>(T G \times V)_{F L}$, suggesting that the grain size at $\mathrm{P} 2$ is finer than that at $\mathrm{P} 1$.

As a welding speed increases, the weld pool becomes longer and narrower (teardrop shape), and in contrast, the weld pool becomes elliptical as the welding speed decreases [9]. A 
teardrop weld pool geometry produces smaller $(T G / V)_{C L}$ and $(T G \times V)_{C L}$ than an elliptical weld pool. Therefore, the microstructure of the teardrop weld pool is more dendritic/equiaxed and finer at the fusion line than the microstructure of the elliptical weld pool.

\subsection{Welding Parameters}

The welding power density distribution and the welding speed (welding parameters), as well as the configuration of the weld joint and the thermal properties of the material, determine the shape of a weld pool. The weld joint configuration and material can be controlled during the design stage, and the welding power and speed can be controlled during the welding process. However, in practice solidification parameters cannot be controlled directly, though they can be indirectly controlled by the welding parameters. Controlling welding parameters influences the shape of the weld pool and determines the solidification parameters. Finally, the solidification parameters control the microstructure chemical composition and grain size and shape.

The cooling rate is critical to controlling weld microstructure. Mathematically, the cooling rate is the thermal gradient multiplied by the growth rate in differential form (i.e. $\frac{d T}{d t}=\frac{d T}{d x}$.

$\frac{d x}{d t}$. As the cooling rate increases, the microstructure becomes finer and more dendritic for the particular welding job. In practice, the cooling rate can be controlled by the welding speed, heat input, preheating, and weldment transverse thickness (heat sink). 


\subsection{Solidification Microstructure}

Many industrial products are the result of the solidification process, directly or indirectly. As the properties of the products are determined by their microstructure, controlling the microstructure controls the products properties. The simulation of microstructure evolution in solidification is a key factor to understanding, controlling, and improving solidification microstructures. The most common solidification morphologies in practical alloys are dendrites and equiaxed. Important solidification microstructure features include the tip radius of the dendrites $\left(R_{t i p}\right)$, the arm spacing of the dendrites $\left(\lambda_{1}\right)$, the equiaxed grain size, and the secondary arm spacing $\left(\lambda_{2}\right)$, as depicted in Figure-1.6a. The size and component segregation of these microstructure features determine the properties.

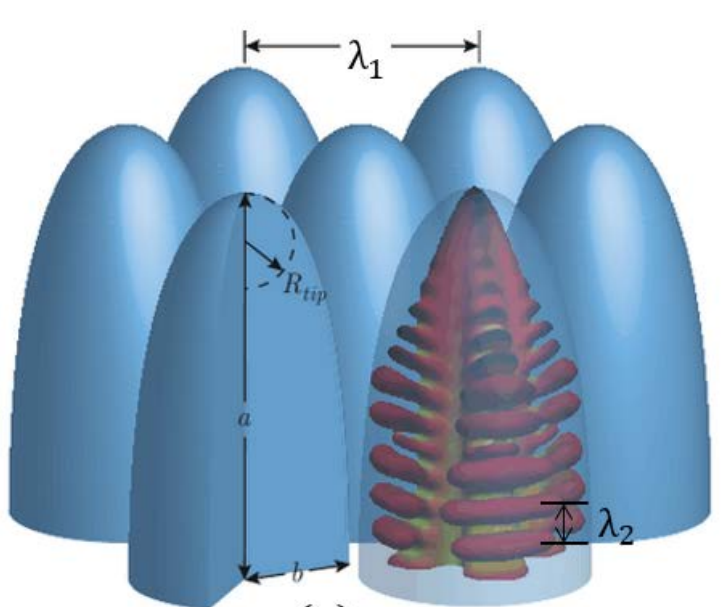

(a)

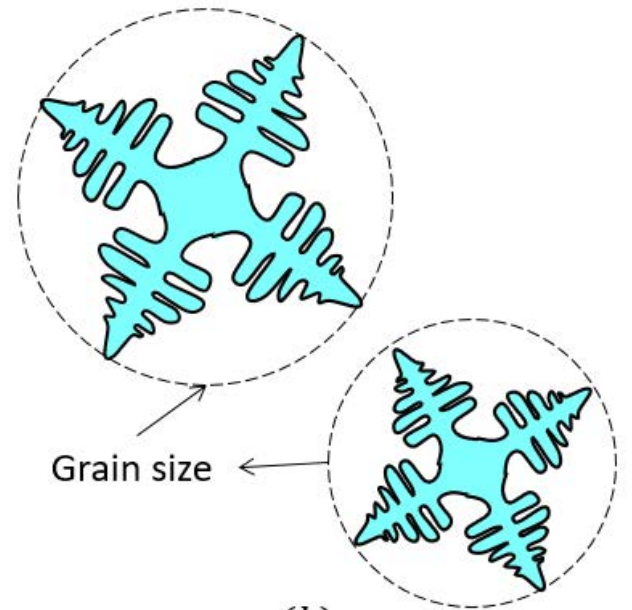

(b)

Figure-1.6: (a) The main microstructure features, displaying the model of the dendrite envelope as an ellipsoid. [11], page-323. (b) the size of equiaxed grain.

The dendritic arm spacing is related to the mechanical properties of the alloy; for example, smaller arm spacing increases mechanical properties. The primary spacing depends on the 
solidification velocity and temperature gradient, $\lambda_{1}=\operatorname{const} \cdot\left(V \cdot T_{G}\right)^{-n}$ [12]. Since the product $V \cdot T_{G}$ is the cooling rate, an increased cooling rate results in finer microstructure. In addition, the secondary arm spacing $\left(\lambda_{2}\right)$ depends on the solidification time $\left(t_{f}\right)$, and $\lambda_{2}=\left(\mu_{o} \cdot t_{f}\right)^{n}$, where $n=0.33$ to $0.5, \mu_{o}$ is a function of alloy constituents and $t_{f}$ is the solidification time [12]. As the solidification proceeds, some dendrites grow, and others stop to grow because of the competitive growth. In addition, the larger arms grow at the expense of the smaller ones, and this is known as the dynamic coarsening or ripening of dendrites. Consequently, solidification time, $\lambda_{1}$, and $\lambda_{2}$ determine the grain structure that, in return, establishes the mechanical properties of the solidified microstructure or weld.

\subsubsection{Solidification Driving Force}

Thermodynamically, the driving force of the solidification process is the change in free energy. During solidification, free energy change can be achieved by cooling a liquid phase and introducing some degree of undercooling, $\Delta T$. Thus, the undercooling is the driving force of microstructure evolution. The total undercooling of a solidifying cell can be split into four parts [14]: solutal undercooling $\Delta T_{c}$, thermal undercooling $\Delta T_{t h}$, capillarity undercooling $\Delta T_{r}$, and attachment kinetics undercooling $\Delta T_{k}$ (see Figure-1.7). Kinetic undercooling can be neglected under normal solidification conditions for metals and alloys.

$$
\Delta T=\Delta T_{t h}+\Delta T_{c}+\Delta T_{r}+\Delta T_{k} \quad E q 1.1
$$




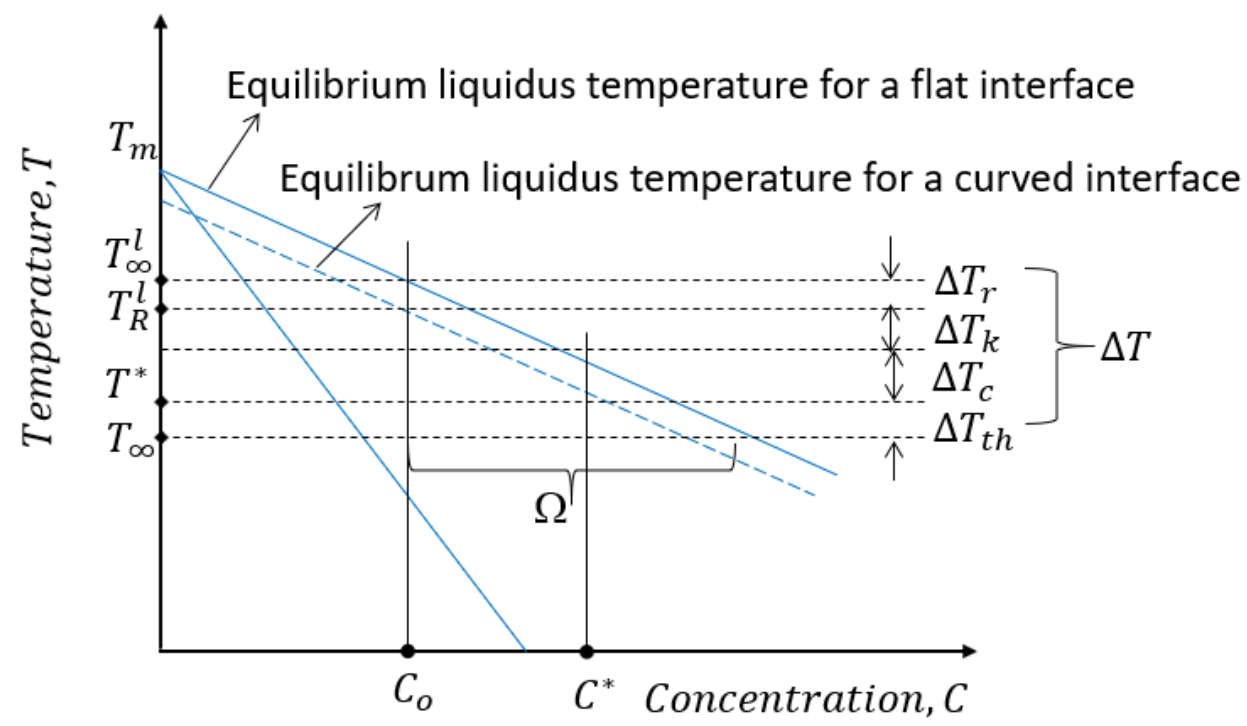

Figure-1.7: The components of undercooling.

In Figure-1.7, $T_{m}$ is the melting temperature of the host component at a flat interface, $T_{m}^{R}$ is the melting temperature at a curved interface, $T_{m}^{K}$ is the melting temperature because of the kinetics, $T^{*}$ is the interface temperature, $T^{\infty}$ is the temperatures of the far field liquid, $C_{o}$ is the nominal alloy composition, and $\Delta C_{0}$ is the supersaturation. During the solidification of an alloy, the heat and solute atoms are rejected at the S/L interface and form thermal and solute layers. The extent and gradient of the two layers determine the morphology and the segregation of the microstructure. In addition, due to the capillarity effect the curvature of the $\mathrm{S} / \mathrm{L}$ interface affects the microstructure evolution. Therefore, the interplay of thermal, solute, and capillary is the solidification mechanism that controls the final microstructure. While, the capillarity effect tends to flatten the S/L interface (stabilize the interface) to reduce the total interfacial energy, the solutal layer tends to amplify any perturbation on the interface (destabilize the interface), thereby producing a complex 
structure. The thermal layer can stabilize the interface (flatten it) if its gradient is positive and destabilizes the interface if its gradient is negative.

\subsubsection{The Capillarity Effect}

The curvature of an $\mathrm{S} / \mathrm{L}$ interface has a significant effect on solidified microstructure morphology, since the curvature increases or decreases the melting temperature of the interface locally. These changes in the equilibrium melting temperature affect the total undercooling which, in subsequently, affects the driving force of the solidification. This curvature effect can be quantified using the Gibbs-Thomson relation, which states that the melting temperature of a curved interface is equal to the equilibrium melting temperature minus the Gibb-Thomson coefficient, multiplied by the curvature of the interface, as in:

$$
T_{m}^{k}=T_{m}^{e}-\Gamma \cdot K \quad E q 1.2
$$

where the Gibb-Thomson coefficient $(\Gamma)$ is approximately equal to $\frac{\text { surface energy }}{\text { entropy of fusion }} \approx 10^{-7}$ for most metals [12]. Conventionally, a convex interface (i.e. a bump of solid phase) has a positive curvature, and a concave interface has a negative one. Therefore, the melting temperature is suppressed at the tip of any perturbation (convex curvature), and the equilibrium melting temperature is increased at any valley (concave curvature), as shown in Figure-1.8a. The effect of curvature on equilibrium melting temperatures can be explained by thermodynamics. As Gibbs free energy is proportional to the interfacial energy, if an interface area increases (e.g. the area of a convex perturbation), the Gibbs free energy curve moves up as shown in Figure-1.8b. This shift in the Gibbs energy of solid 
decreases the equilibrium melting temperature, and the opposite occurs in the case of concave curvature. The change in curvature values is complex when a curved interface advances during solidification. Sharp curvatures tend to flatten the $\mathrm{S} / \mathrm{L}$ interface because perturbations tend to melt, and depressions tend to solidify and flatten.

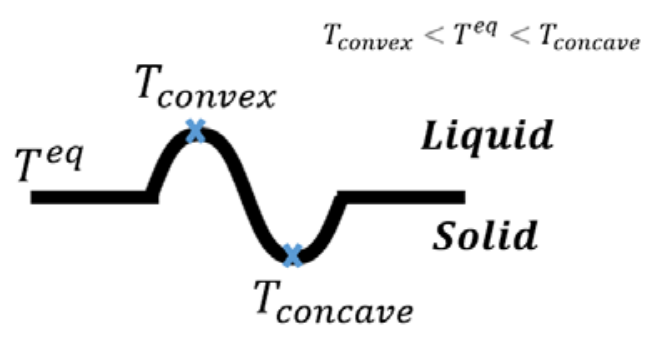

(a)

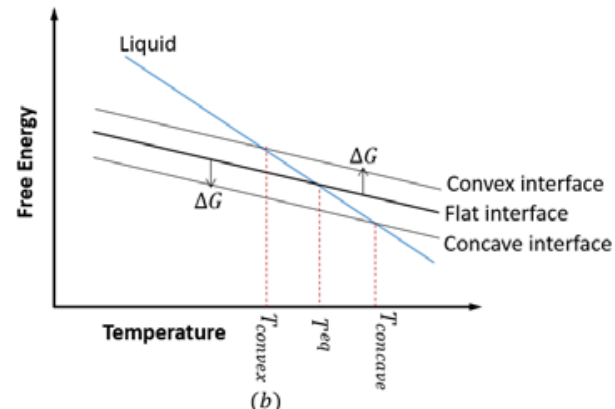

(b)

Figure-1.8: The effect of curvature on the melting temperature. $T^{e q}$ is the equilibrium melting temperature of a flat interface, $T^{\text {convex }}$ is the melting temperature of a convex interface, and $T^{\text {concave }}$ is the melting temperature of a concaved interface.

\subsubsection{Analytical Dendrite Growth Models}

A full-scale dendrite is a complex structure with a non-steady state evolution and developing analytical models for this problem is unlikely. However, some proposed analytical models attempt to describe the tip growth kinetics and the tip size, as determined by the thermal and solutal fields as well as capillarity. Most of the analytical models investigate the growth of only the dendrite tip which is considered to be spherical or paraboloid, see Figure-1.9.

One of the first models regards a dendrite as needle-like crystal with a hemispherical cap (hemispherical approximation), as shown in Figure-1.11a. This model solves the steady 
state diffusion equation in radial coordinates and the solution yields the following relationship [12]:

$$
\frac{V \cdot r}{2 D}=\frac{C^{l}-C_{o}}{C^{l}-C^{s}} \quad \text { or } P_{C}=\Omega_{C} \quad \text { Eq1.3 }
$$

where, $P_{C}$ is the solutal Peclet number, $\Omega_{C}$ is the solutal dimensionless supersaturation, $V$ is the tip growth velocity, $r$ is the tip radius, $C_{o}$ is the initial alloy composition, $C^{l}$ is the solute concentration in liquid, $C^{S}$ is the solute concentration in solid, and $D$ is the diffusivity coefficient. Similar relationship can also be obtained for a thermal dendrite. Equation-1.3 indicates that the growth velocity depends on the tip radius and supersaturation. Hemispherical model assumes spherical symmetry of the concentration field around the dendrite tip. This assumption leads to the equal radial growth rate at all points at the hemispherical tip and should produce continuously increasing tip radius during the dendrite growth. This makes a steady state growth with constant tip radius impossible contrary to experimental observation [12].

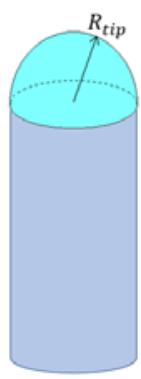

(a)

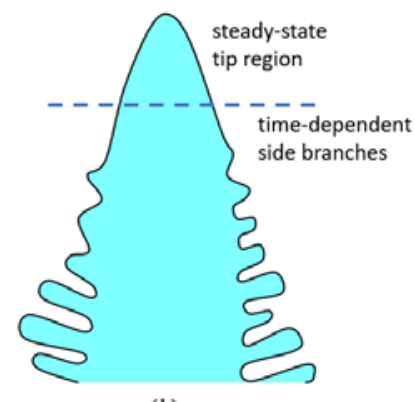

(b)

Figure-1.9: (a) hemispherical model and (b) paraboloid of revolution model. 
The growth of a dendrite (see Figure-1.9b) produces dendrite tip region with a steady-state shape. In contrast, the remainder of the dendritic interface grows in an unstable and timedependent manner, producing quasi-periodic waves that become side arms, or branches.

Papapetrou [15] studied the solidification of salt crystals experimentally. The main outcomes of his theses include: dendrites evolve from small spherical crystals, a dendrite tip has the form of a paraboloid of revolution, and surface tension plays an important role in determining the tip size and arm spacing. Motivated by Papapetrou's results, Ivantsov [15] assumed the dendrite tip has the shape of a paraboloid of revolution, which is selfpreserving shape. Ivantsov's solution of the steady state diffusion equation considering a parabolic shape is given by:

$$
I v\left(P_{C}\right)=\Omega_{C} \quad I v\left(P_{C}\right)=P_{C} \cdot \exp \left(P_{C}\right) \int_{P}^{\infty} \frac{\exp (-x)}{x} d x \quad E q 1.4
$$

Ivantsov's solution is valid for both the solutal diffusion $\left(P_{C}\right.$ and $\left.\Omega_{C}\right)$ and the thermal $\operatorname{diffusion}\left(P_{T}\right.$ and $\left.\Omega_{T}\right)$ in which $P_{T}=\frac{V \cdot r}{2 \alpha}$ and $\Omega_{T}=\frac{\Delta T_{T}}{H_{f} / C p}$.

Both the hemispherical model and Ivantsov's solution conclude that a steady state predicts the tip kinetics will follow a simple scaling law $V \cdot r=$ constant. This states that the tip radius is inversely proportional to its growth velocity. Thus, a dendrite with a small tip radius has higher growth velocity than one with a larger tip radius. However, this prediction has two problems. First, as the tip radius approaches zero the growth velocity increases to infinity. Second, there is no unique solution for $V$ and $r$, because, mathematically, there 
are two unknowns and one equation. Temkin [15] solved the first problem by including the capillarity effect in Ivantsov's model, so the tip radius cannot reach zero because the capillarity will blunt a too sharp tip. The most common solution to the second problem is the marginal stability assumption.

The marginal stability hypothesis was introduced in [16] by performing a linear stability analysis of a parabolic dendrite tip region. If a smooth $\mathrm{S} / \mathrm{L}$ interface is distributed by periodic solid bumps with a certain wavenumber $\lambda_{W}$, the interface will become smooth if its wavenumber is less than the critical wavenumber $\lambda_{c r}: \lambda_{W}<\lambda_{c r}$. In contrast, the solid bumps will grow forming side branches if its wave number is greater than the critical wavenumber: $\lambda_{W}>\lambda_{c r}$. However, the interface will be marginally stable if $\lambda_{W}=\lambda_{c r}$. The marginal stability hypothesis assumes that the tip radius is equal to $\lambda_{c r}$, and this condition can be applied to Ivantsov's solution to predict a unique solution for the tip radius $R_{t i p}$ and velocity $V$. Lipton-Glicksman-Kurz model (LGK model) [17] used the marginal stability hypothesis and produced two equations that can be solved simultaneously to estimate $R_{\text {tip }}$ and $V$. The application of marginal stability predicts that a growing dendrite with $R_{\text {tip }}<\lambda_{\text {cr }}$ will become slower and blunt its tip. On the other hand, a growing dendrite with $R_{\text {tip }}>\lambda_{c r}$ will grow faster and sharpen/split its tip.

\subsubsection{Nucleation}

Solidification consists of two stages: nucleation and growth. Nucleation involves small nuclei of the solid phase that grow or melt depending on its volume and surface energy. 
The volume free energy change $\Delta G_{v}$ is the difference between the Gibbs energy of the solid phase and the liquid phase, and the value of $\Delta G_{v}$ is negative when the temperature is below the equilibrium melting temperature. The surface energy contribution $G_{\gamma}$ is associated with the forming solid surface, and it is always positive. The two energy contributions, $\Delta G_{v}$ and $G_{\gamma}$, compete with each other as follows:

$$
\Delta G=N_{v} \cdot \Delta G_{v}+N_{s} \cdot G_{\gamma} \quad E q 1.5
$$

where, $N_{v}$ is the volume of the forming nucleus and $N_{S}$ is its surface. Thus, if $\Delta G$ is negative, the negative contribution of $N_{v} \cdot \Delta G_{v}$ is greater than $N_{S} \cdot G_{\gamma}$, and the forming solid nucleus will grow. In contrast, if $\left|N_{s} \cdot G_{\gamma}\right|>\left|N_{v} \cdot \Delta G_{v}\right|$, the forming solid nucleus will melt. Therefore, there is a critical nucleus size above which the forming nucleus will grow. The competition between $\Delta G_{v}$ and $G_{\gamma}$ can be illustrated graphically in Figure-1.10.

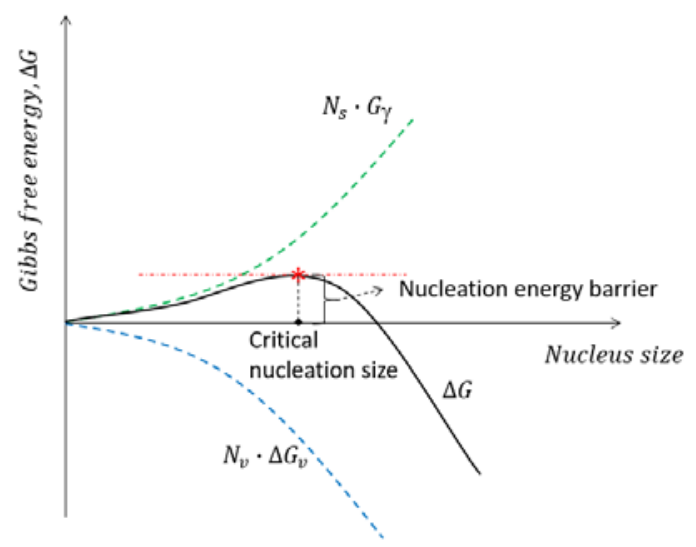

Figure-1.10: Critical size for nucleation.

There are two types of nucleation mechanisms: homogeneous and heterogeneous. With heterogeneous nucleation, the nuclei form at a pre-existing solid surface such as the weld 
substrate. Conversely, with homogeneous nucleation the nuclei of the new phase form in its melt bulk. Both these nucleation mechanisms depend on the degree of undercooling. Heterogeneous nucleation requires less undercooling than homogeneous, because the contribution of surface energy is smaller. Therefore, heterogeneous nucleation is the major nucleation mechanism in welding. However, some weld center line grains (i.e. homogenously nucleated grains) can form under certain welding conditions, including low welding speed and an elliptical weld pool. The degree of undercooling determines the nucleation rate and grain size. Although stable nuclei form at a higher undercooling, the diffusivity of atoms in liquid decreases, resulting in low growth rate. Consequently, the solidification microstructure is finer at a higher undercooling since the nucleation rate is high and the growth rate is low.

\subsubsection{Surface Energy Anisotropy}

The propagation of an $\mathrm{S} / \mathrm{L}$ interface requires a net flow of atoms attached to the interface, and in metals, this attachment produces a rough $\mathrm{S} / \mathrm{L}$ interface. This atomistic interface roughness produces a high surface energy which is orientation dependant, $\gamma(\bar{n})$, and causes a variation in the growth rate of the dendrite according to the crystallographic direction. For BCC and FCC crystals, the higher surface energy is associated with $<100>$ directions [9]. On the other hand, if the surface energy is equal in all crystallographic directions (i.e. isotropy), the resulting dendrites will resemble coral-like structures, see Figure-1.11b. 


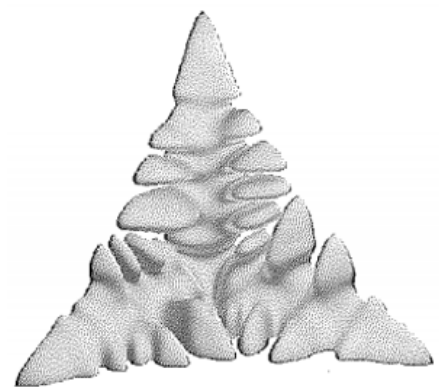

(a)

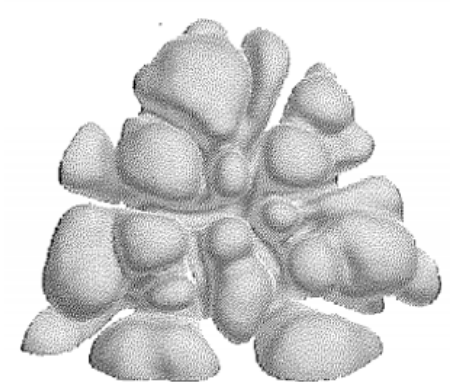

(b)

Figure-1.11: Crystal growth with (a) anisotropic Surface energy (dendrite), and (b) isotropic Surface energy (coral-like). [11], page-317.

Since anisotropy influences crystal morphology during solidification, its effect should be included in the microstructure modeling, and the capillarity undercooling should be modified to incorporate the anisotropy effect. Including the effect of anisotropy in the the capillarity undercooling calculation is given by Gibbs-Thomson-Herring relationships [18]:

$$
\Delta T_{r}=\Gamma \cdot\left(1-15 \epsilon_{4} \cdot \cos 4 \theta\right) \cdot K \quad[\text { Gibbs }- \text { Thomson - Herring }] \quad E q 1.6
$$

where $\Gamma$ is the Gibbs-Thomson coefficient, $K$ is the $\mathrm{S} / \mathrm{L}$ curvature, $A(\bar{n})=$ $\left(1-15 \epsilon_{4} \cdot \cos 4 \theta\right)$ is the anisotropy function, $\epsilon_{4}$ is the four fold anisotropy coefficient, $\bar{n}$ is the local surface normal vector, and $\theta$ is the angle between $\bar{n}$ and the direction of maximum surface energy. Regarding to Equation-1.6, if the anisotropy is weak $\left(\epsilon_{4}<\right.$ $1 / 15)$, the growth is dendritic. In contrast, if the anisotropy is strong $\left(\epsilon_{4}>1 / 15\right)$, the growth is facet [11]. Therefore, $\epsilon_{4}$ can be any value between zero and 1/15 for metals. During the solidification simulation, if an interfacial cell is located along an easy growth 
direction, it will accumulate more solid fraction in a time step. Therefore, the growth will be faster along the easy growth directions.

In the case of $3 \mathrm{D}$, the anisotropy of surface energy is described using two angles as shown in Figure-1.12. Therefore, Gibbs-Thomson-Herring relationship is more complicated, and according to [18] the capillarity undercooling is:

$$
\Delta T_{r}=\Gamma \cdot\left[\left(\gamma(\hat{n})+\frac{\gamma^{2}(\hat{n})}{\partial \theta_{z}^{2}}\right) \cdot K+\left(\gamma(\hat{n})+\frac{\gamma^{2}(\hat{n})}{\partial \theta_{x y}^{2}}\right) \cdot K\right] \quad E q 1.7
$$

where, the anisotropy function is:

$\gamma(\hat{n})=(1-3 \varepsilon) \cdot\left[1+\frac{4 \varepsilon}{1-3 \varepsilon}\left(\cos ^{4} \theta_{z}+\sin ^{4} \theta_{z} \cdot\left(1-2 \sin ^{2} \theta_{x y} \cdot 2 \cos ^{2} \theta_{x y}\right)\right)\right] \quad E q 1.8$

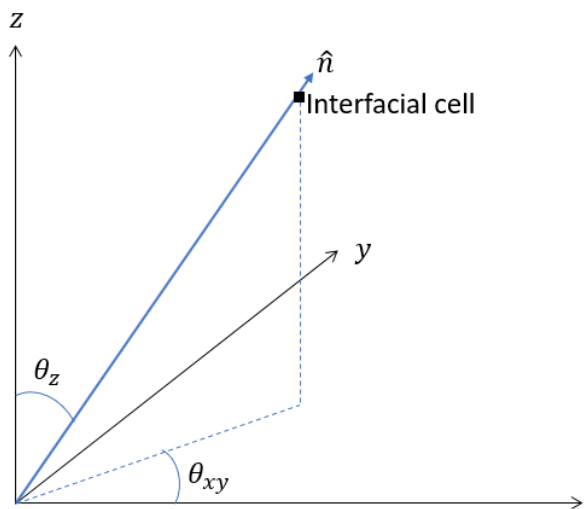

Figure-1.12: The angles that describe the anisotropy in 3D.

\subsection{Solidification Modeling}

Physical solidification problems occur when phase changes transform a liquid phase into a solid phase. Mathematically, this is known as a moving boundary or Stefan problem, after the pioneer work of Stefan. Phase change problems are nonlinear and difficult to solve due 
to discontinuity at the interface between phases. Considering mathematics, the discontinuity indicates that the solution of the underlying partial differential equation (PDE) is not continuous through the entire computational domain. Physically, the interface indicates that the material properties (e.g. density) behind the interface is different than those ahead of interface. Moreover, as the interface is moving with time, its position is not known a priori and depends on the solution. Stefan worked with the solidification of ice [19], and he solved the heat transfer equation in both phases (ice and water) separately, then combined the two solutions by the heat balance condition at the interface, or Stefan condition. Stefan condition assumes that the temperature at the interface is constant and equal to the equilibrium freezing temperature. Thus, the difference of heat flux from the solid phase and the liquid phase does not change the temperature of the interface; rather, it moves the interface with a certain velocity.

Solidification of a material depends on whether it is pure metal or an alloy. In both cases the latent heat is generated at the $\mathrm{S} / \mathrm{L}$ interface and alter the thermal field around the interface. With alloy solidification, there is a compositional field or a compositional difference in solid and liquid phases due to solute rejection or absorption. The compositional difference is indicated in the alloy phase diagram. Both fields (thermal and compositional) drive the kinetics advance of the $\mathrm{S} / \mathrm{L}$ interface, and ultimately determine the interface morphology and hence the entire microstructure. Mathematically, the composition difference should be applied as a boundary condition at the moving interface. Since the concentration field at the boundary influences the boundary movement, the 
problem becomes complex. In addition, more complexity exists because the thermal properties such as thermal conductivity, density, and specific heat are temperature dependant. Thus, due to these complexities, solidification problems are nonlinear and difficult to solve. However, some analytical solutions for one-dimensional pure metals solidification problems have been proposed [11]. All analytical solutions assume a definite shape of the $\mathrm{S} / \mathrm{L}$ interface such as flat, spherical, or paraboloid. For practical solidification modeling, i.e. modeling 3D alloys solidification, numerical schemes should be applied. The modeling can be conducted on different length scales as shown in Figure-1.12.

Referring to Figure-1.13, on a macroscopic scale, the grain structures and macrosegregation can be predicted for a scale of $\mathrm{cm}$ to $\mathrm{m}$. On a mesoscopic scale, grain structures, cellular-to-equiaxed (CET) structure transition can be predicted for a length scale of $\mathrm{mm}$. The dendritic growth morphology and microsegregation can be predicted on a microscopic scale. Finally, the $\mathrm{S} / \mathrm{L}$ interface kinetics and morphology can be predicted on a nanoscopic scale.

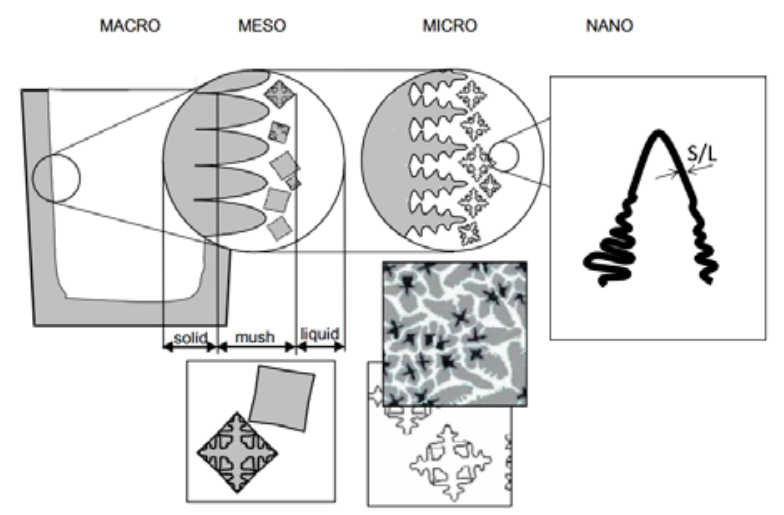

Figure-1.13: Solidification modeling length scales. [12], page-2. 


\subsubsection{Macroscopic Solidification Modeling}

On a macroscopic scale the solidification of a material can be computed by the enthalpy method, in which the phase change and the evolution of the latent heat is accounted for by solving the enthalpy form of heat transfer equation:

$$
\rho \frac{\partial H}{\partial t}=\nabla \cdot\left(k_{t h} \cdot \nabla T\right) \quad[\text { enthalpy formulation] } \quad E q 1.9
$$

where, $\rho$ and $k_{t h}$ are the density and thermal conductivity. With the enthalpy method, the relationship between the enthalpy and temperature $(\mathrm{H}-\mathrm{T})$ should be determined a priori (Figure-1.14). The main advantage of this method is that the solution is valid for the entire computational domain (i.e. both the solid and the liquid phases, and the mushy region). Therefore, the discontinuity at the $\mathrm{S} / \mathrm{L}$ interface is resolved mathematically.

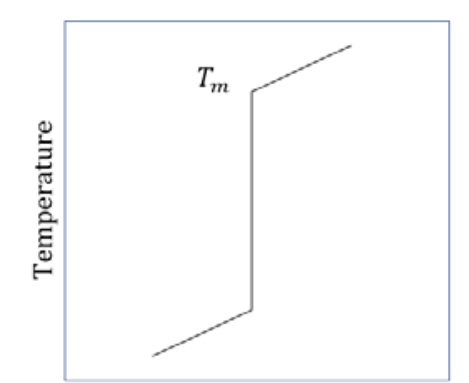

Enthalpy

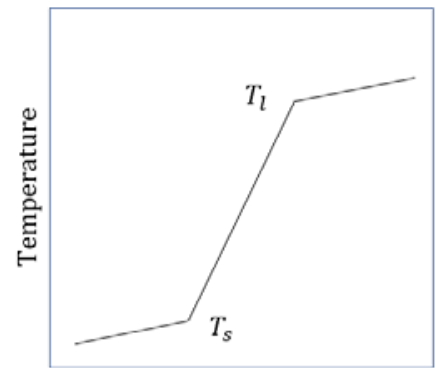

Enthalpy

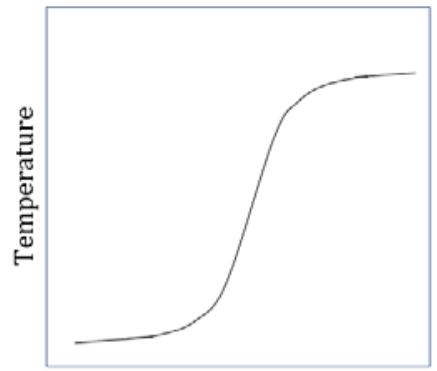

Enthalpy

$$
T(H)=\left\{\begin{array}{cccc}
\frac{H}{C_{p}} & \text { if solid } & H<C_{P} \cdot T_{m} \\
T_{m} & \text { if mushy } & C_{P} \cdot T_{m} \leq H \leq C_{P} \cdot T_{m}+L_{h} \\
\frac{H-L_{h}}{C_{P}} & \text { if liquid } & H>C_{P} \cdot T_{m}+L_{h}
\end{array} \quad T(H)=\left\{\begin{array}{ccc}
\frac{H}{C_{P}} & \text { if solid } & H<C_{p} \cdot T_{s} \\
\frac{H-f^{s} \cdot L_{h}}{C_{P}} & \text { if mushy } & C_{p} \cdot T_{s} \leq H \leq C_{p} \cdot T_{t}+L_{h} \\
\frac{H-L_{h}}{C_{p}} & \text { if liquid } & H>C_{p} \cdot T_{l}+L_{h}
\end{array}\right.\right.
$$

(a)

(b) (c)

Figure-1.14: (a) enthalpy function for pure materials, (b) alloys, (c) real alloys. [13]

Initially, temperature $T^{t}$ and enthalpy $H^{t}$ are assigned to the entire computational domain at the first time step. By applying cooling boundary conditions, at the next time step $t+$ 
$\Delta t$, the new enthalpy $H^{t+\Delta t}$ is calculated using Equation-1.9 for the entire domain. Thus, the values of the new temperature $T^{t+\Delta t}$ can be determined by using the relationship between enthalpy-temperature, as shown in Figure-1.14. The enthalpy functions for the solidification of alloys can be obtained using several models describing fraction of solid as function of temperature, as listed in Table-1.

Table-1: Solidification models [13], where, $f^{s}$ is the fraction of solid, $T^{l}$ is the equilibrium liquidus temperature, $T^{m}$ is the melting temperature of the host alloy, $K_{O}$ is the distribution coefficient, $T^{S}$ is the equilibrium solidus temperature, and $T$ is the local temperature.

\begin{tabular}{|c|l|}
\hline Solidification model & \multicolumn{1}{c|}{ Description } \\
\hline$f^{s}=\frac{T^{l}-T}{\left(1-K_{o}\right)\left(T^{m}-T\right)}$ & $\begin{array}{l}\text { Equilibrium solidification model considering the lever } \\
\text { rule in with complete mixing of solute in both liquid } \\
\text { and solid. }\end{array}$ \\
\hline$f^{s}=1-\left(\frac{T^{m}-T}{T^{m}-T^{l}}\right)^{\frac{1}{K_{o}-1}}$ & $\begin{array}{l}\text { Scheil solidification model in which a complete mixing } \\
\text { of solute in liquid and no mixing in solid are assumed. }\end{array}$ \\
\hline$f^{s}=\frac{T^{l}-T}{T^{l}-T^{s}}$ & $\begin{array}{l}\text { Linear distribution of latent heat of freezing. This } \\
\text { model is assumed if } f^{s} \text { cannot easily be evaluated as a } \\
\text { function of temperature. }\end{array}$ \\
\hline
\end{tabular}

The models in Table-1 consider two approximations that are a constant distribution coefficient $\left(K_{0}\right)$ and a linear liquidus line. The linearization approach increases the uncertainty in solidification modeling, particularly for higher component alloys. To avoid such simplifications, more accurate thermodynamics model is necessary. This study suggests that more accurate model can be achieved by coupling CALPHAD with solidification modeling. In this case CALPHAD is used to obtain the equilibrium 
information for the $\mathrm{S} / \mathrm{L}$ interface. The main advantage of this is the ability to model alloys with non-linearized phase diagrams, since the data of the phase diagram can be obtained for any temperature and composition from the CALPHAD calculations. In addition, multicomponents alloys can be modeled in the same manner as binary alloys, and the benefits of coupling CALPHAD can be achieved at any scale of solidification modeling.

\subsubsection{Mesoscopic Solidification Modeling}

At the mesoscale, a boundary envelope is considered for each grain in the computational domain, and the growth kinetics of each grain envelope is computed for the envelope, regardless of the internal features of the grain, as shown in Figure-1.15.

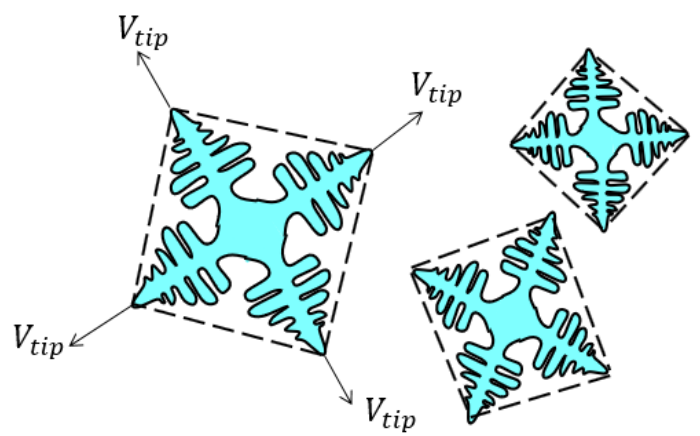

Figure-1.15: Grain envelope geometry used in the mesoscale solidification modeling.

The model in Figure-1.15 has two steps: nucleation and growth. In the nucleation step, the number of grains is determined by a nucleation model that relates the grain density and the undercooling, $\dot{n} \propto \Delta T$, with a specific distribution. Once grains are nucleated, they grow according to the growth kinetics model. The kinetics describes the movement of the envelope tips, and the cellular-to-equiaxed transition can be predicted, which is very important for solidification microstructure properties. Figure-1.16 shows the prediction 
ability of the mesoscale CA for microstructure. Typically, if a parallel computation is used the size of the computational domain in mesoscale modeling can be from millimeters up to a few centimeters [20]. Some references describe using CA at this scale as classical CA modeling [13].

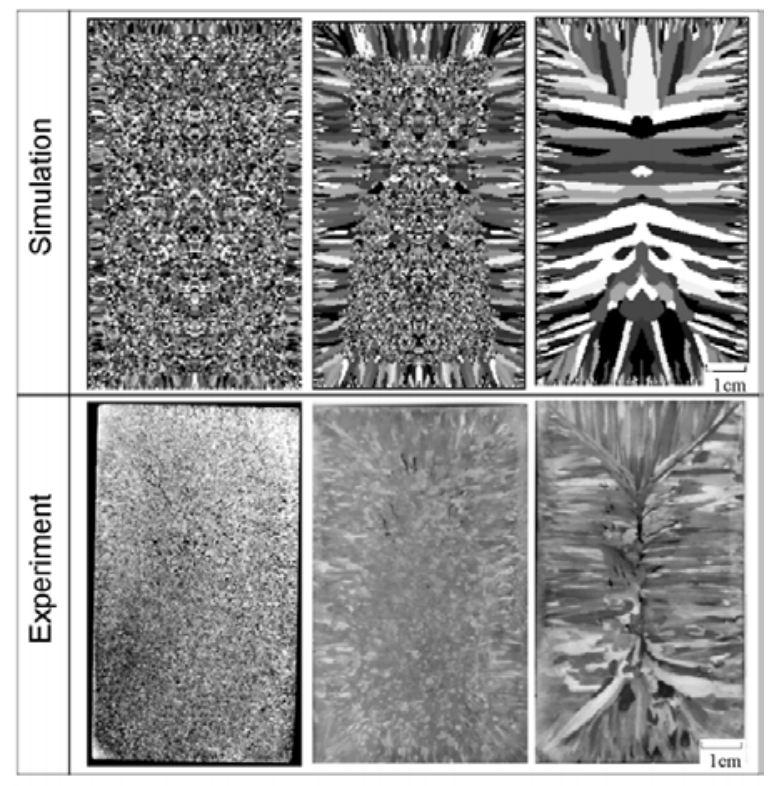

(a)

(b)

(c)

Figure-1.16: An experimental and mesoscale CA simulated macrostructures of an Al$4.5 \% \mathrm{Cu}$ alloy in casting with various pouring temperatures: (a) $710^{\circ} \mathrm{C}$, (b) $760{ }^{\circ} \mathrm{C}$ and (c) $810^{\circ} \mathrm{C} .[13]$, page- 231.

Originally, the cellular automaton (CA) algorithm was developed by John von Neumann to model complex physical phenomena using simple laws [21]. The first numerical modeling of a dendritic structure evolution was performed by Umantsev et al in 1986 [15]. In the nineties, Rappaz et al applied the CA algorithm to simulate the solidification of a cast microstructure [22]. The general procedure of the CA algorithm consists of four steps: (1) subdivide the computational domain into cells and predetermine the initial state; (2) 
specify the state variables to be calculated for each cell (e.g. temperature, crystallographic orientation, solid, liquid); (3) specify the definition of the neighborhood cells; and (4) specify the transition or capturing rules that determine whether a cell will change its state or not.

\subsubsection{Microscopic Solidification Modeling (Cellular Automata Modeling)}

Microscopic CA modeling has the same four basic steps as mesoscale modeling. Unlike mesoscale CA, the main interest with microscopic modeling is the internal microstructure features such as side branching and microsegregation. Therefore, the effect of curvature should be included into the microscopic model. In this case, the values of the curvature of every interfacial cell are considered to be state variables, which means, the effect of capillarity undercooling should be incorporated in the CA model. In addition, the microsegregation within every cell can be computed, which is helpful when studying the microstructure quality as a homogenous microstructure is advantageous. The location of the $\mathrm{S} / \mathrm{L}$ interface is not explicitly defined in CA modeling, but it is implicitly defined by the solid fraction values in all cells. Using today's computational capabilities, the size domain of the microscopic CA modeling has been defined as between 0.1 to $1 \mathrm{~mm}^{3}$.

In microscopic CA modeling, changes in the solid fraction of an interfacial cell can be estimated using the kinetics of the $\mathrm{S} / \mathrm{L}$ interface. The kinetics of the interface, or the velocity of the advanced solid $\left(V^{s / l}\right)$, can be calculated using the Stefan condition [23] or kinetics equation [3]. 


$$
V^{s / l} \cdot C_{l}^{*}\left(k_{o}-1\right)=\left(-D_{l}\left(\frac{\partial C_{l}}{\partial x}+\frac{\partial C_{l}}{\partial y}+\frac{\partial C_{l}}{\partial z}\right)+D_{s}\left(\frac{\partial C_{s}}{\partial x}+\frac{\partial C_{s}}{\partial y}+\frac{\partial C_{s}}{\partial z}\right)\right) \cdot \bar{n} \quad E q 1.10 a
$$

[solute conservation at the interface]

$$
\begin{gathered}
\left.V_{x}^{s / l}=\frac{k_{s}\left(\frac{\partial T}{\partial x}\right)_{s}-k_{l}\left(\frac{\partial T}{\partial x}\right)_{l}}{L_{h}} \quad \text { [heat balance at the interface }\right] \quad E q 1.10 b \\
V^{s / l}=\mu_{k} \cdot \Delta T_{k} \quad[\text { Kinetics equation }] \quad E q 1.11
\end{gathered}
$$

Both models are applied by maintaining the equilibrium at the interfacial cells and using the linearized approach of the phase diagrams. This study uses CALPHAD calculation to impose the equilibrium on the interfacial cells, without computing the kinetics of the $\mathrm{S} / \mathrm{L}$ interface. Thus, for any alloy (binary or $n$-order) CALPHAD can estimate the solid fraction as a function of cell temperature and compositions: $f^{s}=F\left(T^{s / l}, C_{1}^{s / l}, C_{2}^{s / l}, \cdots C_{n}^{s / l}\right)$.

\subsubsection{Nanoscopic Solidification Modeling (Phase Field Modeling)}

At this small length scale, the resolution should be small enough to capture the thickness of $\mathrm{S} / \mathrm{L}$ interfaces $1-10 \mathrm{~nm}$. Although phase-field modeling of the $\mathrm{S} / \mathrm{L}$ interface can produce more accurate microstructure predictions, it is computationally expensive. The phase-field method is used to model solidification and evolving microstructures in materials. The approach is based on the Gibbs free energy minimization principle. The first component of the phase field modeling is to describe the state of a system by the $\operatorname{order}$ parameter $\emptyset(x, t)$, which is a function of position and time. The value of the order parameter defines the state of any point in the system. For example, if a system has two states (i.e. solid and liquid in 
the case of solidification), any point with an order parameter of - 1 is in the solid phase, and any point with an order parameter of +1 is in the liquid phase. The next component is to define a free energy density function $(f)$ for the system. Free energy density is a local variable that represents the free energy of a phase under current thermodynamic conditions, and it is dependent on the state of the system at each point, $f[\varnothing(x, t)]$. The free energy of a two-phase system is comprised of three energy terms that represent the energy that corresponds to the volume of the first phase, the second phase, and the interface. If we ignore the free energy of the interface, the total free energy of a system is the sum of the volume of each phase multiplied by the free energy density of the phase. The final component in phase field modeling is to define the total free energy functional $(G)$ for the entire system domain $(\Omega)$ as follows:

$$
G=\int_{\Omega} f[\varnothing(x, t)] d x \quad E q 1.12
$$

In fact, the free energy density depends on the values of its order parameter $(\varnothing)$ and gradient $(\nabla \emptyset)$, and the width and energy of an interface depends on the gradient of the order parameter. Every point has an order parameter value between -1 and $+1(-1<\emptyset<+1)$, that corresponds to the interface. Thus, the interface can be included in Equation-1.12 as shown in Equation-1.13.

$$
G=\int_{\Omega} f[\varnothing(x, t), \nabla \emptyset(x, t)] d x \quad E q 1.13
$$


Considering that the free energy decreases as $\varnothing$ changes with time, and expanding Equation-1.13 by Taylor series with a second order truncation yields the functional free energy as:

$$
G=\int_{\Omega}\left[f(\varnothing)+\frac{\epsilon}{2}|\nabla \emptyset|^{2}\right] d x \quad E q 1.14
$$

where $\epsilon$ is a function of the interface orientation. If we consider a variation of the functional free energy, a partial differential equation for the evolution of $\emptyset$ can be obtained as:

$$
\frac{\partial \emptyset}{\partial t}=-M \frac{\delta G}{\delta \emptyset} \quad E q 1.15
$$

where $M$ is a kinetics coefficient. The free energy density should be defined as $\emptyset=+1$ for liquids and $\emptyset=-1$ for solids. Caginalp [24] proposed the following expression for the free energy density function:

$$
f(\varnothing)=\frac{1}{8}\left(\varnothing^{2}-1\right)^{2}-2 T \varnothing \quad E q 1.16
$$

where $\varnothing$ of the solid is equal to -1 and $\varnothing$ of the liquid is equal to +1 at $T=0$. Another model of the free energy density function suggested by Karma and Rappel [25] has the following form:

$$
f(\varnothing)=\frac{\emptyset^{4}}{4}-\frac{\emptyset^{2}}{2}+\lambda \cdot T \cdot \varnothing\left(1-2 \frac{\emptyset^{2}}{3}+\frac{\emptyset^{4}}{5}\right) \quad E q 1.17
$$


Kobayashi [26] introduced another form of the free energy density function, and many other researchers also proposed models of the function. Although these models were attempts to mimic the behavior of actual thermodynamic systems, more accurate free energy expressions can be determined using CALPHAD.

Unlike cellular automata, using phase field method precisely defines the interface between solid- and liquid phases and its finite thickness, which resolves the discontinuity problem of the diffusion equation at a sharp interface, as shown in Figure-1.17. However, since the interface is finite and thin, the mesh size must be too small in order to represent the interface. If too fine mesh is used, a costly computationally solution or complex meshadaptive technique is required.

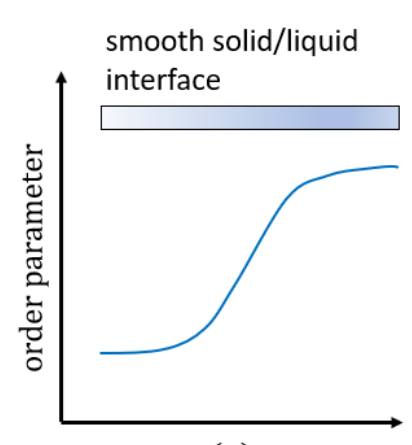

(a)

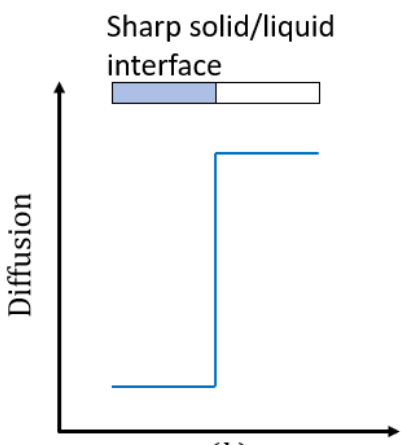

(b)

Figure-1.17: (a) continuous interface, (b) sharp interface.

To summarize, phase field modeling is a successful tool to reproduce dendritic growth features including tip growth behavior, coarsening, crystallographic orientations, and the motion of grain boundaries during impingement. The method describes the $\mathrm{S} / \mathrm{L}$ interface as a continuous function, thereby avoiding the discontinuity and tracking of the interface. 
The PF method requires a very fine grid definition to smoothly define the $\mathrm{S} / \mathrm{L}$ interface, leading to very high computational cost. This limits the simulation to a single dendrite or a very small computational domain. In contrast CA model can use a coarser grid which is more efficient computationally than the phase field model. In [27] a comparison study was conducted to compare 2D CA and $\mathrm{PF}$, using the same simulation parameters to predict a 2D dendrite growth with a single nucleus and multiple arbitrary-oriented nuclei. The study found that both PF and CA were capable to predict the growth with good agreement. A. Choudhury et al [28] evaluated the performance of PF and CA, and concluded that a hybrid method of CA and PF could be developed that combines the efficiency of CA and the accuracy of PF. This method is expected to be useful for the investigation of remelting and fragmentation in the late stages of solidification.

\subsection{CALPHAD vs. The Linearization Approach of Phase Diagram}

According to the literature, cellular automata-based solidification models apply linearized approximation to describe the solidus and liquidus lines of an alloy [23, 13, 29]. This means that a linear equation can be used to determine the relationship between the equilibrium temperature and the concentration. The linearization approach is accepted for any alloy that exhibits slightly curved S/L boundaries. Figure-1.18 shows the aluminum rich corner of Al-Cu and Al-Si binary phase diagrams. As both these systems have a near-linear liquidus line, knowing the concentration of the alloy and slope of liquidus line, allows the temperature to be calculated by the linear relation. The resulting temperature is used to 
calculate the kinetics of a moving $\mathrm{S} / \mathrm{L}$ interface. If a system has more than two chemical components, such as a Al-Cu-Si ternary system, we assume that the liquidus slope of Al$\mathrm{Cu}$ in the binary system is equal to the liquidus of $\mathrm{Al}-\mathrm{Cu}$ in the ternary system, and that the liquidus slope of $\mathrm{Al}-\mathrm{Si}$ in the binary system is equal to the liquidus slope of Al-Si in the ternary system.

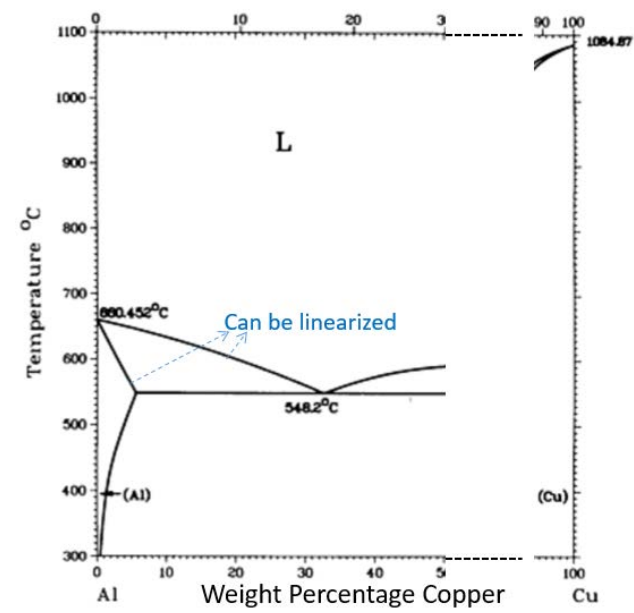

(a)

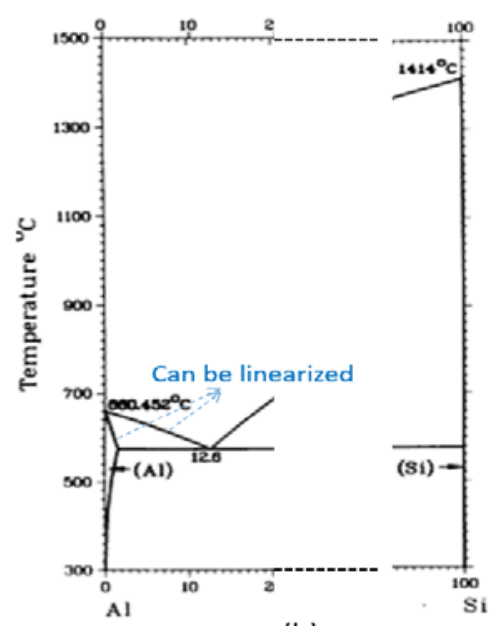

(b)

Figure-1.18: Linearization approach of (a) Al-Cu binary system and (b) Al-Si binary system. [30], pages-286, 316.

This linearization approach involves two uncertainties. The first is approximating the $\mathrm{S} / \mathrm{L}$ as straight lines, even if they are slightly curved. The second uncertainty is considering the behavior of a ternary system (e.g. Al-Cu-Si ternary) as two independent binary systems Al$\mathrm{Cu}$ and $\mathrm{Al}-\mathrm{Si}$, which does not consider any interaction between $\mathrm{Cu}$ and $\mathrm{Si}$. With higher order alloys we consider the equilibrium temperatures and compositions of any two of the components equivalent to those of their binary systems. This adds uncertainty to the solidification model, since the behavior of a single component in a binary system is 
different than its behavior in a higher order alloy. Consequently, as the number of components in an alloy increases the uncertainty of the solidification model also increases.

Linearization approximation of some important binary systems is invalid, because of the high curvature of the S/L lines. For example, in the Fe-Cr binary system shown in Figure1.19, both solidus and liquidus curves cannot be approximated by straight lines. Therefore, the solidification of this binary alloy cannot be investigated using the linearization approach. Using CALPHAD overcomes this limitation and increases the accuracy of the microstructure prediction, and it also has the potential to study any practical alloy. This study couples CALPHAD with CA to study Fe-Cr-C stainless steel alloy.

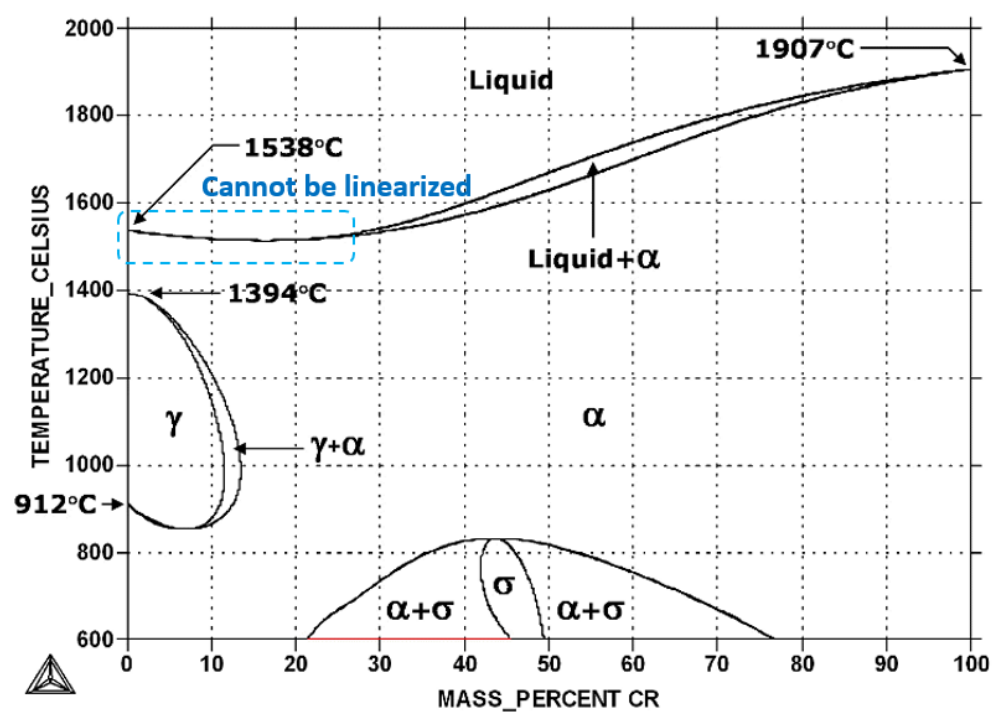

Figure-1.19: Fe-Cr binary phase diagram. 


\section{Chapter-2: The Computation of Phase Diagram (CALPHAD)}

\subsection{Thermodynamics Potentials}

In thermodynamics, the term 'system' is used to identify the subject of the analysis. The system is distinguished from its surroundings by a fixed or moving boundary, and it interacts with its surrounding through the boundary by exchanging energy and/or matter. If the system can exchange both energy and matter with its surroundings, it is known as an open system. The system is called a closed system if it can only exchange energy, and it is known as an isolated system if it cannot exchange energy or matter. The properties of a given system can be extensive, intensive, or partial [31]. Extensive properties depend on system size such as volume (V) or composition (n), intensive properties do not depend on system size such as temperature $(\mathrm{T})$ and pressure $(\mathrm{P})$, and partial properties, such as chemical potentials $(\mu)$, are molar properties. Natural variables, or independent variables, are selected to describe the macrostate of a system, and a given macrostate can be represented by a number of different microstates. Essentially, the microstate of a system is the specific arrangement of the energy of each constituent in the system.

The thermodynamic state of a system can be described by thermodynamic potentials or fundamental functions. Internal energy (U), Gibbs energy (G), Enthalpy (H), and Helmholtz energy (F) are four different potential energies. Each of which is defined by its own natural variables. For example, temperature, pressure, and composition are the natural 
variables for Gibbs energy, $G(T, P, n)$. All thermodynamic properties of a system can be calculated if its energy potential is expressed as a function of its natural variables, which can be done by taking partial derivatives of the energy potential with respect to one of its natural variables. Chemical potentials, for example, can be calculated from Gibbs energy as $\mu=\left(\frac{\partial G}{\partial n}\right)_{T, P}$.

\subsection{Internal Energy (U) and Gibbs Energy (G)}

The internal energy of a system defined in respect to some reference state is equal to the sum of energy added or removed by heat, work, and matter.

$$
U=Q-W+G \quad[\text { internal energy }] \quad E q 2.1
$$

where, $\mathrm{Q}, \mathrm{W}$, and $\mathrm{G}$ are the energy added or removed by heat, mechanical work, and matter. Each energy term can be described by a conjugate pair (i.e. $Q=T \cdot S, W=P \cdot V, G=\mu$. $n)$ as in Eq-2.2.

$$
U=T \cdot S-P \cdot V+\sum_{i}^{\text {no.C }} \mu_{i} \cdot n_{i} \quad[\text { internal energy with conjugate pairs }] \quad E q 2.2
$$

where, $S$ is the system entropy, $V$ is the system volume, no. $C$ is the number of components in the system, $\mu_{i}$ is the chemical potential of a component $i, n_{i}$ is the quantity of moles of component $i$. By taking the total derivative of $\mathrm{U}$ and eliminating the zero terms, the differential form of Eq-2.2 is as follows: 
$d U=T d S-P d V+\sum_{i} \mu_{i} \cdot d n_{i} \quad$ [the differential form of internal energy $] \quad E q 2.3$

Eq-2.3 is the energy scheme of the combined law of the first and second laws of thermodynamics, with $S, V, n_{i}$ the natural variables for $\mathrm{U}$.

Thermodynamics potentials can be mathematically deduced from each other using the Legendre transformation. For example, using the transformation, allows the internal energy function to be reformulated by replacing $\mathrm{S}$ and $\mathrm{V}$ with $\mathrm{T}$ and $\mathrm{P}$ to introduce Gibbs energy. Mathematically, the terms d(TS) and d(-PV) are subtracted from both sides of Eq-2.3.

$d G=d(U-T S+P V)=-S d T+V d P+\sum_{i} \mu_{i} \cdot d n_{i} \quad[$ Legendre transformation $] \quad$ Eq2.4

This transformation is useful since the variables $\mathrm{T}$ and $\mathrm{P}$ are readily controlled experimentally. According to Eq-2.4, under a constant temperature and pressure, the differential Gibbs energy is equal to

$$
d G=\sum_{i} \mu_{i} \cdot d n_{i} \quad[\text { Gibbs energy }] \quad E q 2.5
$$

From Eq-2.5, the chemical potential of component $i$ is equal to

$$
\mu_{i}=\frac{d G}{d n_{i}} \quad[\text { the chemical potential of component } i] \quad E q 2.6
$$

A thermodynamics system is typically comprised of many subsystems, and if all the subsystems are under the same potential values, the energy potentials of the system are 
subject to the law of additivity. For example, the internal energy of a composite system is equal to the sum of all subsystems' internal energy, if the the subsystems have the same values of potentials $\mathrm{P}$ and $\mathrm{T}$.

\subsection{Metallurgical Thermodynamics}

Metallurgical thermodynamics studies the equilibrium states of materials and maps them in phase diagrams; a piece of material or alloy can be considered to be a closed thermodynamics system. An alloy is a heterogeneous system comprised of several open homogenous subsystems, such as phases or grains, and in this context, grain boundaries and phase boundaries are the system boundaries. According to the law of additivity, extensive properties of an alloy are equal to the sum of the properties in all phases. For example, if a material is composed of many phases $(\emptyset=1,2, \cdots)$ with many components $(i=1,2, \cdots)$, and each phase has its own internal energy $U^{\varnothing}$, entropy $S^{\varnothing}$, and composition $n^{\emptyset}$, the properties of the alloy can be described as

$$
U^{\text {Alloy }}=\sum_{\varnothing} U^{\varnothing} \quad S^{\text {Alloy }}=\sum_{\varnothing} S^{\varnothing} \quad n^{\text {Alloy }}=\sum_{\emptyset} \sum_{i} n_{i}^{\varnothing} \quad E q 2.7
$$

The change in any phase internal energy can be described by Eq-2.3 as:

$d U^{\varnothing}=T^{\varnothing} \cdot d S^{\varnothing}-P^{\varnothing} \cdot d V^{\varnothing}+\sum_{i} \mu_{i}^{\varnothing} \cdot d n_{i}^{\emptyset} \quad[$ the combined law for any phase $] \quad E q 2.8$ 
The Gibbs energy of a phase is described as

$$
G^{\varnothing}=\sum_{i} \mu_{i}^{\emptyset} \cdot n_{i}^{\emptyset} \quad E q 2.9
$$

Accordingly, the Gibbs energy of the system is

$$
G^{\text {Alloy }}=\sum_{\varnothing} \sum_{i} \mu_{i}^{\emptyset} \cdot n_{i}^{\emptyset} \quad E q 2.10
$$

\subsection{General Thermodynamics Equilibrium}

Equilibrium indicates that the properties of a system are time independent, and the system will revert to the equilibrium state if it is disturbed. The thermodynamics extremum principle states that for an isolated system, the entropy has a maximum value at equilibrium, because the entropy can only increase. Alternatively, the equilibrium criteria can be obtained by minimizing any energy function (U, H, F, or $\mathrm{G})$. For example, if a system has constant entropy, volume, and number of moles, the internal energy is minimum at equilibrium. If a two-phase system with $\alpha$-phase and $\beta$-phase is considered, and the combined statement of the first and second law of thermodynamics for the two phases are

$$
\begin{array}{ll}
d U^{\alpha}=T^{\alpha} d S^{\alpha}-P^{\alpha} d V^{\alpha}+\mu^{\alpha} d n^{\alpha} & \text { Eq2.11a } \\
d U^{\beta}=T^{\beta} d S^{\beta}-P^{\beta} d V^{\beta}+\mu^{\beta} d n^{\beta} & E q 2.11 b
\end{array}
$$

The change in internal energy of the entire system can be obtained by using the law of additivity: 


$$
d U^{s y s}=T^{\alpha} d S^{\alpha}-P^{\alpha} d V^{\alpha}+\mu^{\alpha} d n^{\alpha}+T^{\beta} d S^{\beta}-P^{\beta} d V^{\beta}+\mu^{\beta} d n^{\beta}
$$

Substituting the constrains $d S^{\alpha}=d S^{\beta}, d V^{\alpha}=d V^{\beta}$, and $d n^{\alpha}=d n^{\beta}$ into Equation-2.12 gives:

$$
d U^{\text {sys }}=\left(T^{\alpha}-T^{\beta}\right) d S^{\alpha}-\left(P^{\alpha}-P^{\beta}\right) d V^{\alpha}+\left(\mu^{\alpha}-\mu^{\beta}\right) d n^{\alpha} \quad E q 2.13
$$

The minimum internal energy (Equation-2.13) is achieved when all coefficients are equal to zero. This yields to the thermodynamics equilibrium criteria: $T^{\alpha}=T^{\beta}, P^{\alpha}=P^{\beta}$, and $\mu^{\alpha}=\mu^{\beta}$. Therefore, a thermodynamics system is at equilibrium when the temperature, pressure-, and chemical potentials among the system parts are equal.

As constraining entropy and volume is not experimentally practical, using internal energy in developing phase diagrams is difficult, so in this case, Gibbs free energy, in which temperature and pressure are controlled parameters, is used instead. Gibbs energy can be applied as a tool to recognize if a thermodynamics process can occur spontaneously. For spontaneous change between two states, the difference in Gibbs energy should be negative, $\Delta G<0$. The Gibbs energy function can also determine the equilibrium, because its value is minimum at equilibrium. In Gibbs energy formulation, equilibrium is reached if the chemical potentials of all parts in the system are equal, since the temperature and the pressure are constants. 


\subsection{Phase Equilibria and Gibbs Energy}

Metallurgical thermodynamics is a powerful tool for predicting whether an alloy is in equilibrium or not. Phase transformation occurs because the initial state of the alloy is unstable relative to the final state, and phase stability is measured by Gibbs free energy of the alloy. The definition of Gibbs free energy of a system is:

$$
G=U+P V-T S \quad \rightarrow G=H-T S \quad \text { [Gibbs free energy }] \quad E q 2.14
$$

where $\mathrm{H}$ is the enthalpy, $\mathrm{T}$ is the temperature, and $\mathrm{S}$ is the entropy of the system. The term PV in Equation-2.14 can be dropped, as the change in volume is very small when dealing with condensed phases. A system is in equilibrium when it has no need to change, or mathematically when $d G=0$. Therefore, solid phases are stable at low temperatures because the term $\mathrm{H}$ is higher than the term TS. In contrast, liquids become more stable at high temperatures because the term TS dominates.

The relationship between equilibrium and Gibbs energy is shown in Figure-2.1, which shows a schematic variation of Gibbs free energy with atoms arrangement. At configurations A and B the system is in equilibrium, since the change in Gibbs energy is zero. Configuration B is a metastable equilibrium (local minimum) and configuration $\mathrm{A}$ is the stable phase (global minimum). However, at any intermediate configuration, in which $d G \neq 0$, the state is unstable, and the system will move toward configuration $\mathrm{A}$ or $\mathrm{B}$ depending on the available driving force. The necessary criterion for any phase 
transformation is $\Delta G=G_{2}-G_{1}<0$, because the transformation should result in decreased Gibbs energy.

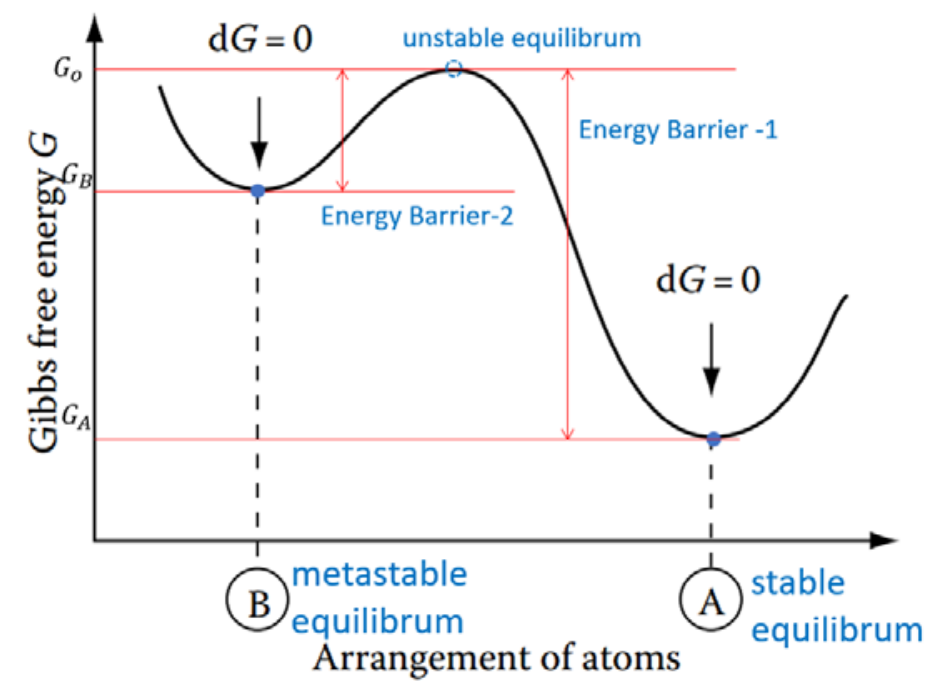

Figure-2.1: The variation of Gibbs free energy with the arrangement of atoms.

\subsubsection{Phase Stability of Metallic Alloys}

A pure material is a single component system consisting of one element or molecule that does not dissociate. Stable phases can be predicted by plotting the variation of Gibbs energy with temperature (G-T). Enthalpy and thermal entropy, the components of Gibbs energy, can be calculated from the specific heat at a constant pressure $(\mathrm{Cp})$, as described in Equation-2.15 and 2.16.

$$
\begin{array}{ll}
H=H_{0}+\int_{298}^{T} C_{p} d T & E q 2.15 \\
S=S_{0}+\int_{0}^{T} \frac{C_{p}}{T} d T & E q 2.16
\end{array}
$$


where, $S_{0}$ is the entropy reference state and $H_{0}$ is the enthalpy reference state. The variation of Gibbs energy with temperature can be illustrated by substituting Equation-2.15 and Equation-2.16 into Equation-2.14.

In alloys, equilibrium involves variables of pressure, temperature-, and composition, and depends on how the Gibbs energy varies according to these variables. In a two-component (binary) alloy at a constant pressure, phase stability can be determined from the variations in Gibbs free energy of the all possible phases, as well as temperature and composition. Gibbs energy variations with composition at a constant temperature can be depicted in energy-composition diagrams.

Figure-2.2d shows a pseudo-isomorphous binary system with liquid and solid phases. If the Gibbs energy of the liquid phase is lower than that of the solid phase for all variations of B, the liquid phase is stable, as illustrated in Figure-2.2a. In reverse, the solid phase will be stable if it has a lower Gibbs energy, as shown in Figure-2.2b. However, when two Gibbs energy curves intersect, the liquid and solid phases coexist in equilibrium with one another and produce a 'mushy zone', as shown in Figure-2.2c. For two phases in equilibrium, there is a common tangent line that touches the two Gibbs curves at two points. These points present the liquidus and the solidus equilibrium composition as in Figure2.2d. Another example is the stability of eutectic systems is shown in Figure-2.3a. At the eutectic temperature $\left(\mathrm{T}_{\mathrm{E}}\right)$, there are three phases in equilibrium: the liquid phase $l$ and two solid phases $\alpha$ and $\beta$. Thus, there is a common tangent line that touches the three curves at 
three distinct points, as in Figure-2.3b. These points represent the equilibrium composition of each phase.

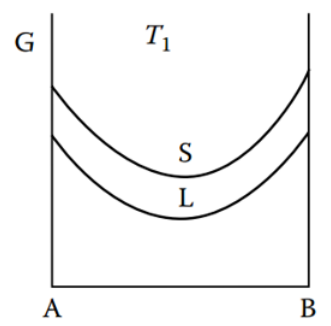

(a)

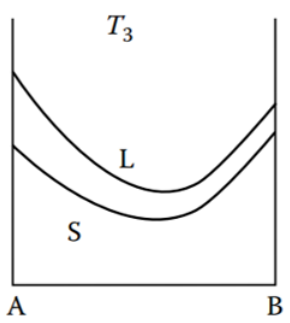

(b)

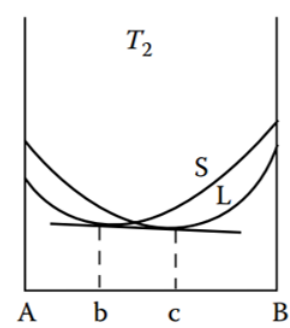

(c)

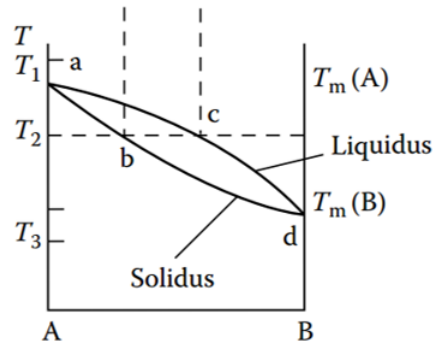

(d)

Figure-2.2: Gibbs energy and the stability of a binary system. [35], page-33.

Another example is the stability of eutectic systems is shown in Figure-2.3a. At the eutectic temperature $\left(\mathrm{T}_{\mathrm{E}}\right)$, there are three phases in equilibrium: the liquid phase $l$ and two solid phases $\alpha$ and $\beta$. Thus, there is a common tangent line that touches the three curves at three distinct points, as in Figure-2.3b. These points represent the equilibrium composition of each phase.

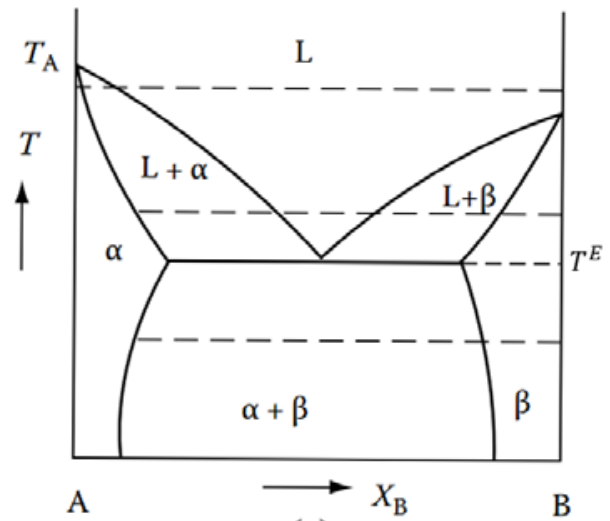

(a)

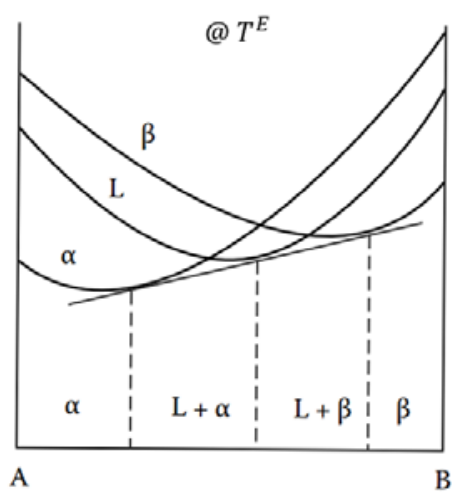

(b)

Figure-2.3: The Gibbs energy curves at eutectic reaction. [35], page-37. 


\subsection{Phases Stability and Chemical Potentials}

Chemical potential determines the inclination of a chemical component to diffuse from one phase to another. If the chemical potential is equal in both phases there is equilibrium and no tendency for the diffusion. For a metallurgical system with two components (A and B), and three phases $(l, \alpha-$, and $\beta)$, there is chemical potential for each component in each phase, denoted as $\mu_{A}^{l}, \mu_{B}^{l}, \mu_{A}^{\alpha}, \mu_{B}^{\alpha}, \mu_{A}^{\beta}, \mu_{B}^{\beta}$. For equilibrium, under constant pressure and temperature, all atoms in the system must not tend to move. Figure-2.4, shows that if the equilibrium state is not achicved, the chemical potentials of atoms A and B in the $\alpha$-phase are not equal to those of the atoms in the $\beta$-phase, as in Figure-2.4a. In this case, atoms $\mathrm{A}$ and B will transfer from one phase to another until the equilibrium state is reached, as shown in Figure-2.4b.
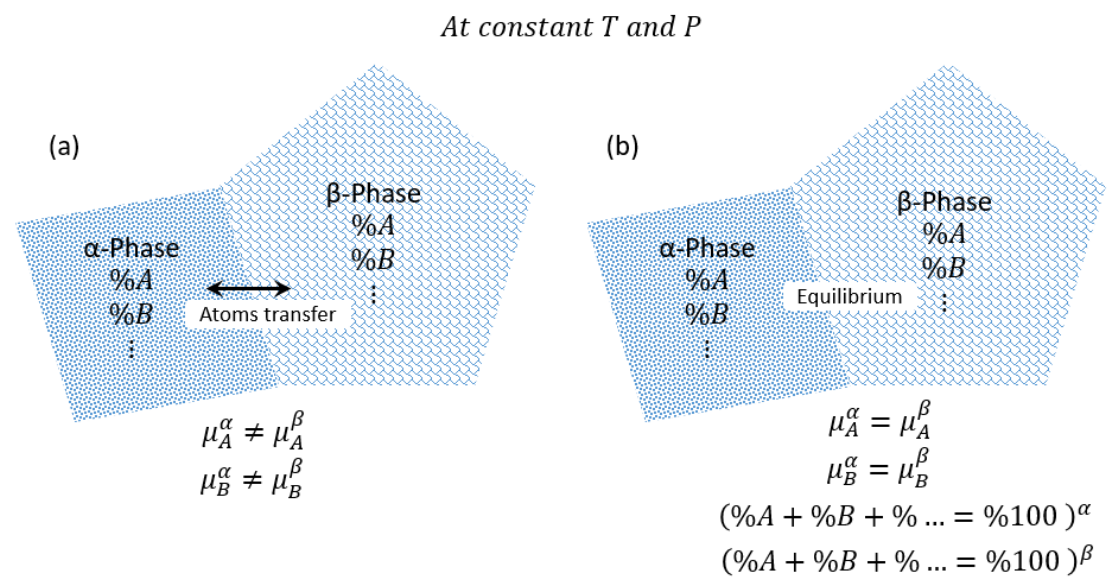

Figure-2.4: Chemical potentials at constant temperature and pressure for (a) nonequilibrium state and (b) Equilibrium state. 
If the temperature or pressure changes, the equilibrium state is altered, and the atoms will transfer between the two phases leading to a new equilibrium state that could produce a different phase.

Chemical potentials can be calculated mathematically from the Gibbs free energy curves. Since Gibbs energy changes when atoms are added or removed, its value is proportional to the amount of the added/removed atoms $(G \propto n)$. For instant, if a small quantity of Aatoms $\left(d n_{A}\right)$ is added at a constant temperature, pressure, and constant number of B-atoms to the system, the proportionality constant is equal to the chemical potential, $d G=\mu_{A}$. $d n_{A}$. Thus, the value of chemical potential can be calculated as:

$$
\mu_{A}=\left(\frac{\partial G}{d n_{A}}\right)_{T, P, n_{B}}
$$

In Equation-2.17, the chemical potential is known as the partial molar free energy. The same procedure is valid if we add B-atoms to the system, and the next expression for the differential Gibbs energy can be written as:

$$
d G=\mu_{A} \cdot d n_{A}+\mu_{B} \cdot d n_{B} \quad E q 2.18
$$

If molar fractions $\left(X_{A}, X_{B}\right)$ are used instead of the quantity of components $\left(n_{A}, n_{B}\right)$, the value of Gibbs energy per mol can be written as:

$$
G=\mu_{A} \cdot X_{A}+\mu_{B} \cdot X_{B} \quad E q 2.19
$$


Therefore, the relationship between Gibbs energy and chemical potentials yields to a relationship illustrated in Figure-2.5. The chemical potential of $\alpha$ solution of a multicomponents system can be expressed as follows [32]:

$$
\mu_{i}^{\alpha}=G^{\alpha}+\frac{\partial G^{\alpha}}{\partial X_{i}}-\sum_{i}^{n} \frac{\partial G^{\alpha}}{\partial X_{i}} \quad \text { [general chemical potential] } \quad E q 2.20
$$

where, $i$ is the number of the component, $X$ is the molar fraction of the component, $G^{\alpha}$ is the molar Gibbs energy of the phase.

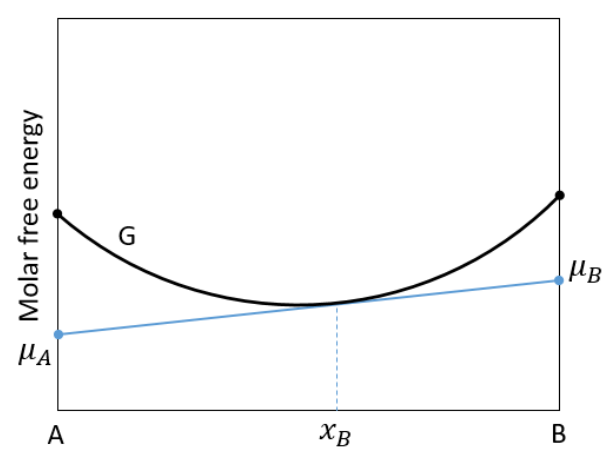

Figure-2.5: The graphical interpretation of chemical potentials in a binary system.

As depicted by the figure, the values of the chemical potentials of elements $\mathrm{A}$ and $\mathrm{B}$ in solution with composition $x_{B}$ are the intersect points of a tangent line, with the Gibbs energy vertical axis at pure A and pure B compositions.

Common tangent lines can be used to predict phases stability, as well as to construct phase diagrams. Figure-2.6 shows two Gibbs energy curves intersecting with. Common tangent intersects of the molar Gibbs energy vertical axis at pure A and pure B atoms at two points, $P 1$ and $P 2$. These intersection points are the values of the chemical potential of each 
component in each phase. The equilibrium is satisfied when the chemical potentials of each component are equal in all phases, $\mu_{A}^{l}=\mu_{A}^{S}$ and $\mu_{B}^{l}=\mu_{B}^{S}$. Figure-2.7 shows the relationships between the common tangent line and stability. At temperature $T_{2}$, the solidus and liquidus lines are defined by points a, b, c, and d. As there are two phases and two Gibbs energy curves, two common tangent lines are required. Since each line touches the energy curves at two points, the four points on the solidus and liquidus lines can be calculated.

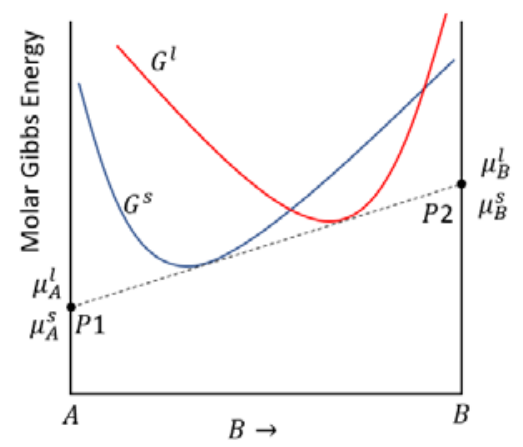

Figure-2.6: The common tangent line and stability.
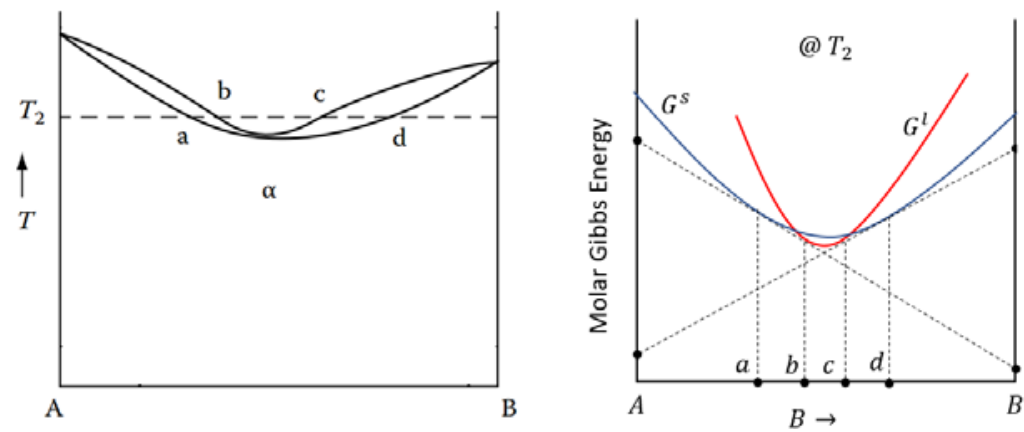

Figure-2.7: Phase stability with two common tangent lines. 


\subsection{Ternary Phase Diagram and Gibbs Energy}

The Gibbs phase rule states that

$$
D_{f}=i-\emptyset+2 \quad[\text { Gibbs phase rule }] \quad E q 2.21
$$

where $D_{f}$ is the degree of freedom or number of independent variables that can be arbitrarily specified to fix a system at equilibrium, $i$ is the number of components, and $\emptyset$ is the number of phases at equilibrium.

Ternary systems are comprised of three components, so they have four independent variables according to the Gibbs phase rule: pressure, temperature-, and two components. The third component is a dependent variable that conserves the mass. Under constant pressure, a ternary phase diagram stores the metallurgical equilibrium information of any alloy that is composed of a given three components and given temperature. The phase diagram information that should be stored in a database are stable phases, the fraction of all phases, and the amount of each component in all phases. The ternary phase diagram can be constructed using the Gibbs triangle or the compositional triangle. By stacking many Gibbs triangles, the entire ternary phase diagram resembles a triangular prism, as shown in Figure-2.8. Each Gibbs triangle (i.e. a "slice" of the prism) is known as isotherm because it stores the information at a specific temperature. The vertices of a given Gibbs triangle represent three pure component metals. In addition, the edges represent binary metals, and each point inside the triangle represents a ternary metal comprised of a mixture of all three components. 


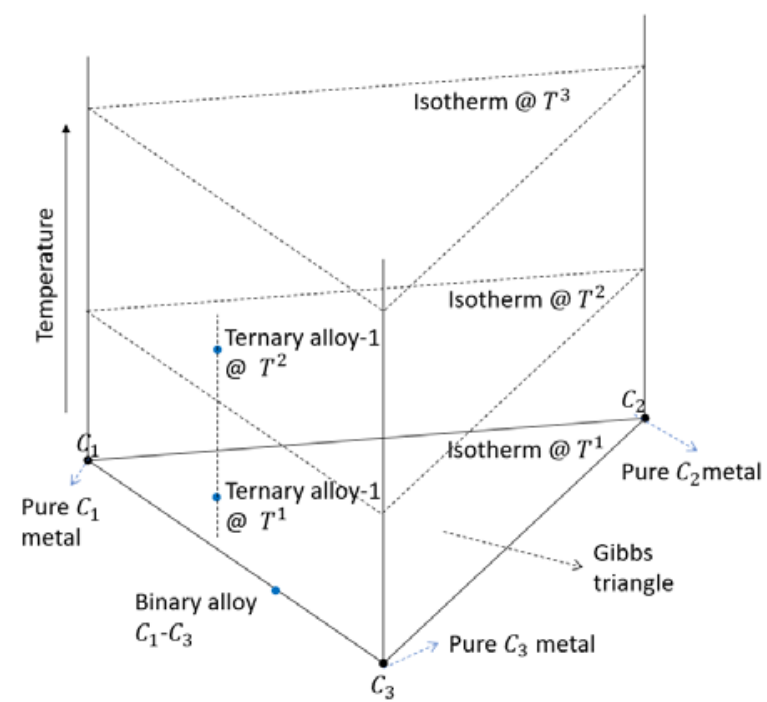

Figure-2.8: The construction of a ternary phase diagram.

Isotherms can be used to read the equilibrium information of any specific alloy composition and temperature, and the two-phase regions appear as a collection of tie lines on the isotherms. For example, Figure-2.9a shows an isotherm with two three distinct zones: liquid single phase $(L)$, solid single phase $(S)$, and two-phase zone $(L+S)$. Any alloy composition located within a single-phase zone has thermodynamic properties of this single phase. In Figure-2.9, for example, the alloy with composition $C_{1}$ is completely liquid as it has a liquid fraction equal to one, and the alloy with composition $C_{2}$ is completely solid as it has a solid fraction equal to one. In the two-phase zone (mushy zone), any alloy composition is described by a tieline belongs to the zone. For example, the alloy composition $C_{0}$ is partially liquid and partially solid and the fraction of solid $f^{s}$ can be obtained by the lever rule. The corresponding liquid fraction $f^{l}$ and solid fraction $f^{s}$ of alloy with composition $C_{0}$ can be calculated as shown in the figure. In addition, the 
intersection point $\left(C_{1}^{l}, C_{2}^{l}\right)$ of the tieline with the liquidus curve describes the concentration of component one and component two in liquid. Similarly, the intersection point $\left(C_{1}^{S}, C_{2}^{S}\right)$ describes the concentration of component one and component two in solid. Thus, any alloy inside the two-phase zone can be described by a different tieline. Unlike binary systems, the equilibrium of three-phase in ternary systems occur over a range of temperatures. The equilibrium of the three phases can be considered a tie triangle, as shown in Figure-2.9b. Each side of the tie triangle is a tie line for a different two-phase region; thus, two-phase regions branch out from the tie triangles.
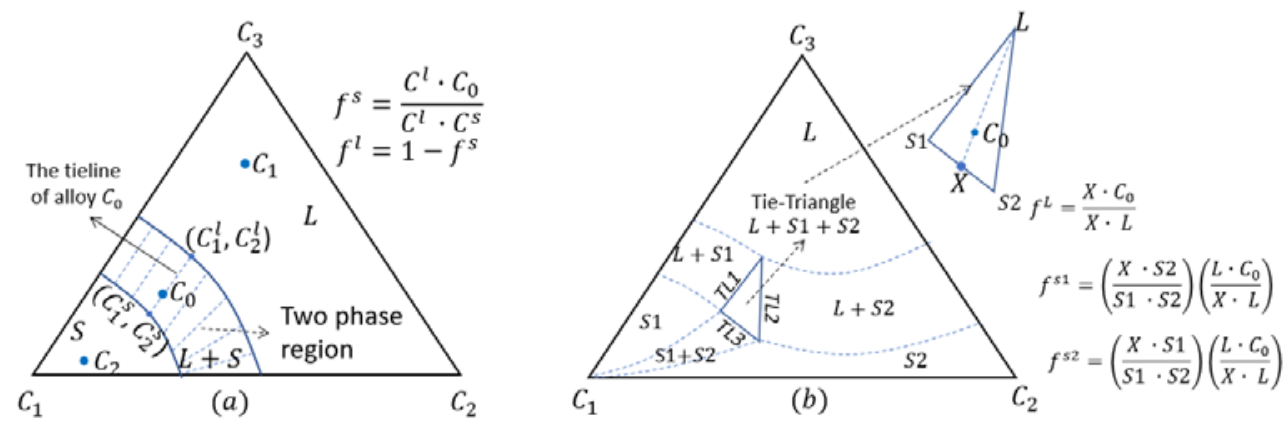

Figure-2.9: Reading equilibrium information from isotherms with (a) tie lines and (b) tie triangles.

For example, side TL1 is the first tie line of the $\mathrm{L}+\mathrm{S} 1$ two-phase region. As shown in the figure, the lever rule can be extended to calculate the equilibrium fraction of each phase. In practice, a full 3D presentation of a ternary system is very complicated to conceive and construct. However, the tie lines and tie triangles together are the basic building blocks from which equilibrium information can be obtained. 


\subsection{Thermodynamics Models for CALPHAD}

The phase diagram of a given alloy system is based on the minimization of its total Gibbs energy $\left(G^{s y s}\right)$, which is equal to the summation of the Gibbs energy of each phase:

$$
G^{s y s}=\sum_{\varnothing=1}^{n o . \varnothing} n^{\emptyset} \cdot G^{\varnothing} \quad[\text { the total Gibbs energy }] \quad E q 2.22
$$

where, $G^{\emptyset}$ is the molar Gibbs energy of the phase $\emptyset$ and $n^{\emptyset}$ is the number of moles.

The Gibbs energy of each phase can be assessed experimentally. The thermodynamic assignment describes the temperature, pressure, magnetic influence, and any other contributions to the Gibbs energy of the assessed phase. Mathematically, the Gibbs energy of any phase $\emptyset$ is described as

$$
G_{T}^{\emptyset}=G^{0}(T, P)+G^{\text {ideal }}+G^{e x}+G_{m a g}^{\emptyset} \quad[\text { the Gibbs energy of } \emptyset \text { phase }] \quad \text { Eq2.23 }
$$

where, $G^{0}$ describes the Gibbs energy of the mechanical mixture of the phase components, $G^{\text {ideal }}$ describes the entropy of mixing, $G^{\text {ex }}$ (the excess Gibbs energy) describes the energy due to the chemical interaction of the components, and $G_{m a g}^{\emptyset}$ is the contribution of the magnetic effect.

Figure-2.10 illustrates the terms in Equation-2.23 for Al-Mg binary system [33]. The first term $G^{0}$ describes the Gibbs energy of the mechanical mixture of the phase components, the second term $G^{\text {ideal }}$ describes the entropy of mixing, and the last term $G^{e x}$, or the excess 
Gibbs energy, describes the energy due to the chemical interaction of the components. The entropy of mixing $\Delta G_{m i x}$ is equal to $G^{i d e a l}+G^{e x}$.

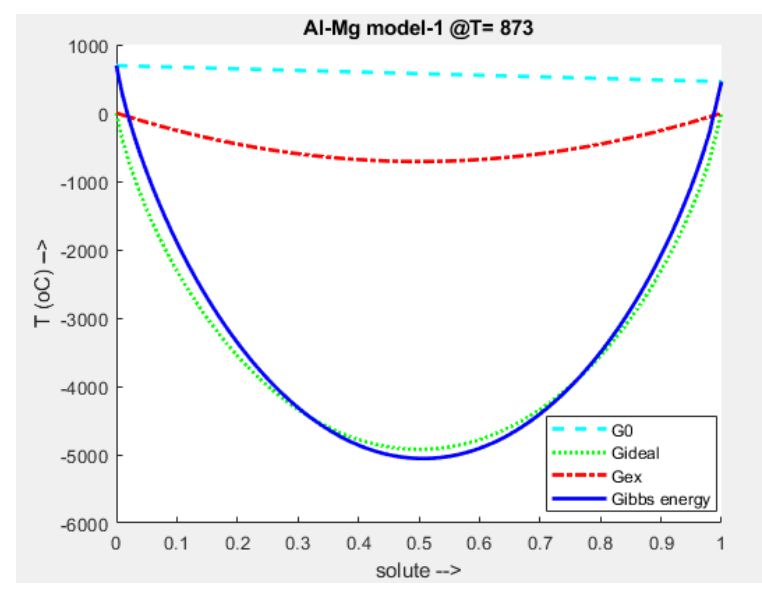

Figure-2.10: The terms in Equation-2.23 for Al-Mg alloy.

For metals, the types of thermodynamics models depend on $G^{e x}$, and in ideal models, $G^{e x}$ is equal to zero. Ideal models can be used to describe gaseous phases. Conversely, for real solution models, $G^{e x}$ can be described by interaction parameters. A real solution model yields to a regular model if $G^{e x}$ is described by one interaction parameters. Regular models can be used to describe liquid metal phases, as well as some solid metal phases. Finally, for complex alloys, the excess Gibbs energy $G^{e x}$ needs more sophisticated descriptions such as the Redlich and Kister polynomial (RK-polynomial) [34].

\subsubsection{Ideal Solution Model}

The ideal solution is the simplest mixing model in which no chemical interaction between atoms is considered, so $\Delta H_{m i x}=0$. Therefore, the Gibbs energy of ideal mixing is due to entropy and mechanical mixing, as follows: 


$$
G=\sum_{i} X_{i} \cdot G_{i}^{o}+R T \sum_{i} X_{i} \cdot \ln X_{i} \cdot \quad \text { [Gibbs energy of ideal solution] Eq2.24 }
$$

where $i$ is the number of components, $X_{i}$ is the molar quantity of component $i$, and $G_{i}^{o}$ is the Gibbs energy of pure component $i$. The term $\left(R \sum_{i} X_{i} \cdot \ln X_{i}\right)$ is the entropy of mixing or $\Delta S_{\text {mix }}$, and it introduces the effect of configurational entropy in equilibrium. For a binary alloy system with two components, A and B, Equation-2.24 reduced to

$$
G=X_{A} \cdot G_{A}^{o}+X_{B} \cdot G_{B}^{o}+R T\left(X_{A} \cdot \ln X_{A}+X_{B} \cdot \ln X_{B}\right) \quad[\text { ideal binary system }] \quad E q 2.25
$$

And the chemical potential of components A and B in the ideal solution can be calculated using the next equation [35].

$$
\begin{aligned}
& \mu_{A}=G_{A}^{o}+R T \cdot \ln X_{A} \quad[\text { chemical potentials of ideal solution] } E q 2.26 \\
& \mu_{B}=G_{B}^{o}+R T \cdot \ln X_{B}
\end{aligned}
$$

As the main assumption of the ideal solution model is that there is no chemical interaction between atoms, it can be used to efficiently model gaseous phases. And, with some modification, introducing activity $(a)$, into the ideal solution model can model dilute solid solutions, since the activities can account for some chemical interactions among atoms.

Activities quantify the tendency of atoms to leave solutions, and measure how the solutions deviate from the ideal behavior. They define the activity coefficient $\gamma$ as the activity divided by the amount of the component, as in $\gamma_{i}=a_{i} / X_{i}$, where $a_{i}$ and $X_{i}$ are the activity and the molar fraction of component $i$, respectively. Thus, the chemical potentials can be defined using activities as: 
$\mu_{A}=G_{A}^{o}+R T \cdot \ln a_{A}$

$\mu_{B}=G_{B}^{o}+R T \cdot \ln a_{B}$

[chemical potentials of ideal solution with activities] Eq2.27

With suitable dilute solutions Henry's law $\left(\gamma_{B}=\right.$ cons $)$ and Raoult's law $\left(\gamma_{B}=1\right)$ can be used to relate the activity to the amount of the component, though regular solution model is more efficient for modeling solid and liquid solutions.

\subsubsection{Regular Solution Model}

An ideal solution model assumes there is no chemical interaction (bonding) between atoms, and the mixing is due to the entropy. As this is unrealistic for liquids and solids, a regular solution model based on quasi-chemical assumptions was introduced. Quasi-chemical model accounts for the bonding energy of the adjacent atoms only so that bond energy is independent of composition. A quasi-chemical assumption can be applied to liquid phases of metals more than solid phases, since the interatomic distances are relatively larger in liquid phases. The enthalpy of mixing $\left(\Delta H_{\text {mix }}\right)$ in a regular solution model can be described using bonding energy as:

$$
\Delta H_{\text {mix }}=Z_{A B} \cdot \epsilon_{\text {mix }} \quad \epsilon_{\text {mix }}=\epsilon_{A B}-0.5\left(\epsilon_{A A}+\epsilon_{B B}\right) \quad \text { [enthalpy of mixing ] } \quad E q 2.28
$$

where, $Z_{A B}$ is the number of A-B bonds in the mixture, $\epsilon_{m i x}$ is the total bonding energy of mixing, $\epsilon_{A B}$ is the energy of A-B bonds, $\epsilon_{A A}$ is the energy of A-A bonds, and $\epsilon_{B B}$ is the energy of B-B bonds. If $\epsilon_{m i x}$ is equal to zero, the regular solution becomes an ideal solution. In the case of a negative $\Delta H_{m i x}$ (i.e. endothermic reaction), the bonding energy of unlike atoms (A-B bonds) is less than the bonding energy of similar atoms (A-A and B- 
B), so $\epsilon_{m i x}<0$. Thus, A-B bonding is more favorable and abundant in the solution. In contrast, for a positive $\Delta H_{m i x}$ (i.e. exothermic reaction), $\epsilon_{m i x}>0$ and similar bonding types are more abundant in the solution. The effect of $\Delta H_{\text {mix }}$ on the $\Delta G_{\text {mix }}$ at high and low temperature is shown in Figure-2.11. At a negative $\Delta H_{m i x}$ and a high temperature (Figure2.11a), there is more mixing between unlike atoms in the solution and so more A-B bonding. In contrast, at a lower temperature (Figure-2.11b) the mixing of A-B atoms is less than the mixing at a higher temperature. On the other hand, at a positive $\Delta H_{m i x}$ and a high temperature (Figure-2.11c), the mixing of different atoms is less favorable than the bonding of similar atoms A-A and B-B. In addition, a positive $\Delta H_{\text {mix }}$ at a low temperature could lead to spinodal decomposition, as shown in Figure-2.11d.
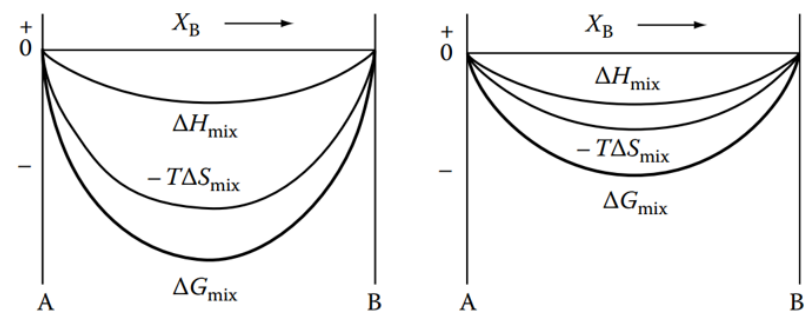

(a) $\Delta H_{m i x}<0, \operatorname{high} T$

(b) $\Delta H_{\text {mix }}<0$, low $T$
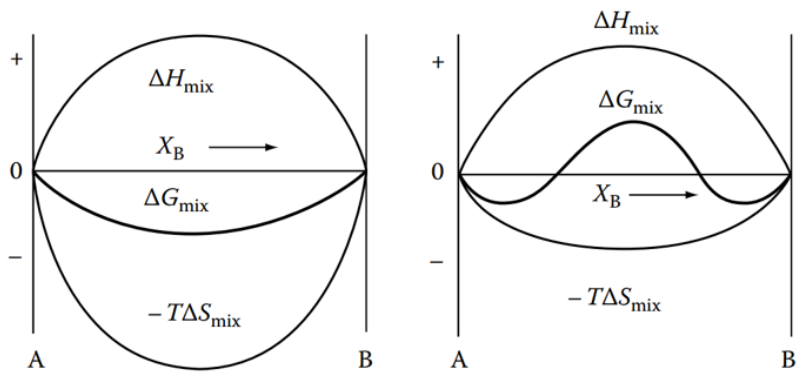

(c) $\Delta H_{m i x}>0$, high $T$

(d) $\Delta H_{\text {mix }}>0$, low $T$

Figure-2.11: The effect of $\Delta H_{\text {mix }}$ on the $\Delta G_{m i x}$ at different temperatures $T$. [35], page21. 
For practical purpose, enthalpy of mixing $\Delta H_{m i x}$ is described by an interaction parameter $(L)$. For an alloy with multicomponents, the excess Gibbs energy is described by:

$$
G^{e x}=\Delta H_{\text {mix }}=\sum_{\substack{i=1 \\ i \neq j}}^{\text {no.C }} X_{i} \cdot X_{j} \cdot L_{i j} \quad[\text { regular solution's excess Gibbs energy }] \quad E q 2.29
$$

The interaction parameter is a function of temperature which often has the following form:

$$
L(T)=a+b \cdot T+c \cdot T \cdot \ln T+d \cdot T^{2}+\cdots \quad E q 2.30
$$

The Gibbs energy of mixing of a regular solution model is defined as:

$$
\Delta G_{\text {mix }}=\underbrace{L_{A B} \cdot X_{A} \cdot X_{B}}_{\Delta H_{\text {mix }}}+\underbrace{R T\left(X_{A} \cdot \ln X_{A}+X_{B} \cdot \ln X_{B}\right)}_{T \Delta S_{\text {mix }}} \quad \text { [for Reular solution ] Eq2.31 }
$$

The chemical potentials of a binary regular solution can be obtained using Equation-2.17 as follows:

$\mu_{A}=G_{A}^{o}+L_{A B}\left(1-X_{A}\right)^{2}+R T \ln X_{A}$

$\mu_{B}=G_{B}^{o}+L_{A B}\left(1-X_{B}\right)^{2}+R T \ln X_{B}$

[regular solution's chemical potentials] $\quad E q 2.32$

\subsubsection{Real Solution Model}

The regular model considers chemical interactions between all atoms and their neighboring atoms only. This assumption does not predict the dependence of $G^{e x}$ on the composition correctly, which limits the feasibility of this model for many practical alloys. To overcome this limitation, Redlich and Kister proposed a polynomial expression to describe the composition dependence of $G^{e x}$ as follows: 


$$
G^{e x}=\sum_{\substack{i=1 \\ j>i}}^{n o . c} X_{i} X_{j} \cdot\left(\sum_{n=1}^{2} L_{i j}\left(X_{i}-X_{j}\right)^{n}\right)
$$

Equation-2.32 suggests that the excess Gibbs energy of a higher order alloy, e.g. A-B-C atoms, can be described as a set of binary alloys (A-B, A-C, B-C), and an interaction parameter for the effect of the three components $L_{A B C}$ can be added to the RK-polynomial if it is assessed experimentally. For a binary system, Equation-2.33 yields to:

$$
G^{e x}=X_{A} X_{B} \sum_{n=1}^{2} L_{A B}\left(X_{A}-X_{B}\right)^{n} \quad\left[G^{e x} \text { for a binary alloy }\right] \quad \text { Eq2.34 }
$$

Clearly, if $n$ equals to 1 , the real model yields a regular model, and describing the Gibbs energy of a phase (as in Equation-2.23) using a real solution model requires a complex mathematical expression. For example, for a ternary system with $\mathrm{A}, \mathrm{B}$, and $\mathrm{C}$ types of atoms, Equation-2.23 yields to:

$$
\begin{aligned}
& G^{\varnothing}(T, X) \\
& =\overbrace{X_{A} \cdot G_{A}^{o}+X_{B} \cdot G_{B}^{o}+X_{C} \cdot G_{C}^{o}}^{=G^{0}}+\overbrace{R T\left(X_{A} \cdot \ln X_{A}+X_{B} \cdot \ln X_{B}+X_{C} \cdot \ln X_{C}\right)}^{=G^{\text {ideal }}} \\
& +\underbrace{X_{A} X_{B} \sum_{n=1}^{2} L_{A B}\left(X_{A}-X_{B}\right)^{n}+X_{A} X_{C} \sum_{n=1}^{2} L_{A C}\left(X_{A}-X_{C}\right)^{n}+X_{B} X_{C} \sum_{n=1}^{2} L_{B C}\left(X_{B}-X_{C}\right)^{n}}_{=G^{e x}} E q 2.35
\end{aligned}
$$

However, Equation-2.35 can simply be considered as a mathematical function that depends on temperature ( $T)$ and composition $\left(X_{A}, X_{B}, X_{C}\right)$. 


\subsubsection{Sublattice Thermodynamics Model}

The real solution model is efficient to describe high order alloys in substitutional solutions. In substitutional solution, all atoms occupy the main lattice sites interchangeably. This would be accurate in systems with small differences in atom size, but if there are larger size differences in atoms, the interstitial solid solutions are energetically favorable. As the real solution does not account for the interstitial and intermetallic bonds accurately, sublattice modeling was developed.

In sublattice models, the lattice of a phase crystal is composed of many sublattices, and the number of sublattices used depends on the crystal complexity and the thermodynamics assessment. Each sublattice has a specific number of sites that can be occupied by specific atom types. This allows the interaction parameters between different atoms in different sublattices to be evaluated and assigned to the Gibbs energy model. The following assumptions are made of sublattice modeling: (1) the amount of components is described by site fractions; (2) there is random mixing on each sublattice, (and no mixing across sublattices); and (3) the total site fractions on each sublattice is equal to one. An illustrative example of sublattice modeling follows.

\subsubsection{An Example of a Sublattice Model}

Assume a ternary alloy with A, B, and C atoms and a vacancy Va, and all constituents distributed over two sublattices $s 1$ and $s 2$, as shown in Figure-2.12. The number of sites available in the first sublattice is nine for atoms A and B. Similarly, the sites available in 
the second sublattice is three for atoms $\mathrm{C}$ and the vacancies. This information can be lumped in the notation: $(A, B)_{9}^{S 1}(C, V a)_{3}^{S 2}$ or equivalently $(A, B)_{3}^{S 1}(C, V a)_{1}^{S 2}$.

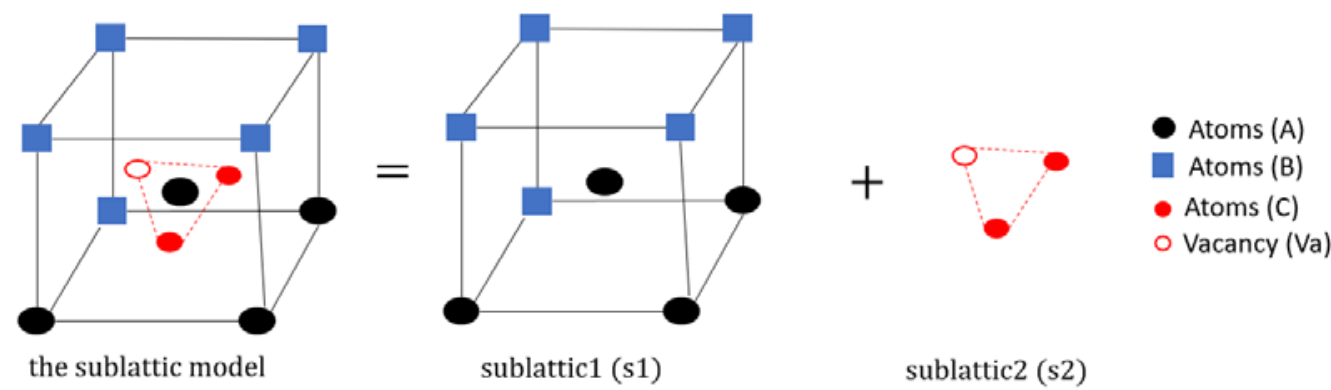

Figure-2.12: An example of a sublattice model.

The site fraction is defined as $y_{i}^{S}=\frac{n_{i}^{S}}{N^{s}}$, where $y_{i}^{S}$ is the site fraction of component $i$ on sublattice $s, n_{i}^{s}$ is the number of components $i$ in sublattice $s$, and $N^{s}$ is the total number of sites on the sublattice $s$. In Figure-2.12, for example, $y_{A}^{S 1}=\frac{4}{9}, y_{B}^{S 1}=\frac{5}{9}, y_{A}^{S}=0$ and $y_{B}^{s 2}=0$. In this case if the sublattice has a vacancy such as s2 in Figure-2.12, the site fraction is defined as:

$$
y_{i}^{S}=\frac{n_{i}^{S}}{n_{V a}^{S}+\sum_{i} n_{i}^{s}} \quad E q 2.36
$$

And for atoms $C$ the site fraction is $y_{C}^{S 2}=\frac{2}{1+2}=\frac{2}{3}, y_{C}^{S 1}=0, y_{V a}^{S 2}=\frac{1}{1+2}=\frac{1}{3}$. The total site fraction in each sublattice should equal 1 to ensure mass conservation. For example, the total site fractions on s1 is $\sum y=\frac{4}{9}+\frac{5}{9}=1$ and on s 2 is $\sum y=\frac{2}{3}+\frac{1}{3}=1$. The relationship between mole fractions and site fractions follows [36]:

$$
x_{i}=\frac{\sum_{s} N^{s} y_{i}^{s}}{\sum_{s} N^{s}\left(1-y_{V a}^{S}\right)} \quad[\text { the relation btween mole and site fractions }] \quad E q 2.37
$$




\subsubsection{The Gibbs Energy in the Sublattice Model}

The Gibbs energy model of an interstitial solution phase with the sublattice model is similar to the model of substitutional model, except the mole fractions are replaced by the site fraction. For example, the Gibbs energy of the sublattice model mentioned in 8.4.1 is as follows [37]:

$$
\begin{aligned}
G^{\varnothing}=y_{A}^{s 1} y_{C}^{s 2 \circ} G_{A: C}+y_{A}^{s 1} y_{V a}^{s 2 \circ} G_{A: V a}+y_{B}^{s 1} y_{V a}^{s 2}{ }^{\circ} G_{B: V a}+y_{B}^{s 1} y_{C}^{s 2 \circ} G_{B: C} \\
+R T\left[a\left(y_{A}^{s 1} \cdot \ln y_{A}^{s 1}+y_{B}^{s 1} \cdot \ln y_{B}^{s 1}\right)+b\left(y_{C}^{s 2} \cdot \ln y_{C}^{s 2}+y_{V a}^{s 2} \cdot \ln y_{V a}^{s 2}\right)\right]+G^{e x}
\end{aligned}
$$

where,

$$
G^{e x}=\sum_{s} \sum_{i} \sum_{j} y_{i}^{s} y_{j}^{s} \cdot \sum_{r \neq s} \sum_{k} y_{k}^{r} L_{i, j, \cdots k} \quad \text { [Gibbs energy of a Sublattice model] } \quad \text { Eq2.38 }
$$

\subsection{Phase Diagram as an Optimization Problem}

Once the thermodynamics assessment is complete for a particular alloy, the construction of its phase diagram can be formulated as a mathematical optimization problem. Specifically, the assessments include the Gibbs energies of all possible phases, the interaction parameters between different components, the definition of sublattices, and the equilibrium range of different phases. The solution of the optimization problem defines the phases boundaries. However, the optimization problem is a non-convex and multiobjective function with linear constraints minimization problem. This is a complex problem especially for multicomponents systems with sublattice modeling. The difficulty of solving these minimization problems arises from the uncertainty in choosing feasible 
initial guesses, as well as from the wide range of decision variables. It is commonly assumed that the correct phases at equilibrium is known a priori, otherwise, the optimization problem would be more difficult. A comprehensive discussion and mathematical formulation of the CALPHAD optimization is in $[38,39]$.

\subsubsection{Global Optimization Formulation of Gibbs Energy Minimization}

Consider a metallurgical system with three components and two phases. The first phase is liquid $l$ with a phase fraction $f^{l}$, and the second phase is solid $s$ with a phase fraction $f^{s}$. The total Gibbs energy of this system is:

$$
G^{\text {sys }}=\sum_{\varnothing}^{l, s} f^{\varnothing} \cdot G^{\varnothing} \rightarrow f^{l} \cdot G^{l}+f^{s} \cdot G^{s} \quad[\text { the total Gibbs energy }] \quad \text { Eq2.39 }
$$

The Gibbs energy of phases can be described by molar fractions $x$ or site fraction $y$, depending on the thermodynamics assessment. The lever rule and the mass balance impose the following constrain on all components in the system:

$$
\sum_{\varnothing}^{l, s} x_{i}^{\varnothing} \cdot f^{\emptyset}=x_{i}^{o} \rightarrow \begin{gathered}
x_{1}^{l} \cdot f^{l}+x_{1}^{s} \cdot f^{s}-x_{1}^{o}=0 \\
x_{2}^{l} \cdot f^{l}+x_{2}^{s} \cdot f^{s}-x_{2}^{o}=0 \\
x_{3}^{l} \cdot f^{l}+x_{3}^{s} \cdot f^{s}-x_{3}^{o}=0
\end{gathered}
$$

where, $x_{i}^{o}$ is the nominal concentration of the component $i$. In addition, the global conservation of phase fractions imposes a constrain:

$$
\sum_{\emptyset}^{l, s} f^{\varnothing}=1 \rightarrow f^{l}+f^{s}-1=0 \quad \text { Eq2.41 }
$$


With regard to the sublattice model, the site fractions balance imposes the next constrain on each sublattice $s l$ :

$$
\sum_{i} y_{i}^{s l}=1 \quad \text { Eq2.42 }
$$

Finally, all system variables $y_{i}^{s l}$ and $f^{\varnothing}$ are in the range between 0 and 1:

$$
\begin{aligned}
& 0 \leq f^{\varnothing} \leq 1 \\
& 0 \leq y_{i}^{\varnothing} \leq 1
\end{aligned}
$$

This mathematical formulation can be solved by applying the Lagrange multiplier method, and thereby transforming the minimization problem of a constraint multivariable function into an unconstrained one objective function optimization problem as follows:

$L\left(x_{i}^{\emptyset}, y_{i}^{s l}, f^{\emptyset}, L_{1}, L_{2}, L_{3}\right)=\sum_{\varnothing}^{l, s} f^{\emptyset} \cdot G^{\emptyset}+L_{1}\left(\sum_{i}^{n o . c} x_{i}^{\emptyset} \cdot f^{\emptyset}-x_{i}^{o}\right)+L_{2}\left(\sum_{\emptyset}^{l, s} f^{\emptyset}-1\right)+$

$L_{3}\left(\sum_{i} y_{i}^{s l}-1\right) \quad$ [Lagrangian function] $\quad$ Eq2.44

where, $L_{1}, L_{2}, L_{3}$ are the Lagrangian multipliers. A set of nonlinear equations is determined by differentiating the Lagrangian function with respect to its variables:

$\frac{\partial L}{\partial x_{i}}, \frac{\partial L}{\partial y_{i}^{S l}}, \frac{\partial L}{\partial f^{\varnothing}}, \frac{\partial L}{\partial L_{1}}, \frac{\partial L}{\partial L_{2}}, \frac{\partial L}{\partial L_{3}}$. Finally, this system of nonlinear equations can be solved by the Newton-Raphson method. Note that the previous procedure should be solved at every temperature value. 


\subsubsection{Phase Diagram from Chemical Potentials Equalities}

Consider two metallurgical systems with two phases (liquid and solid), as shown in Figure2.13. The liquid phase has Gibbs energy diminished by $d G^{l}$, and the solid phase has Gibbs energy diminished by $d G^{s}$. Both systems are composed of components $i$.

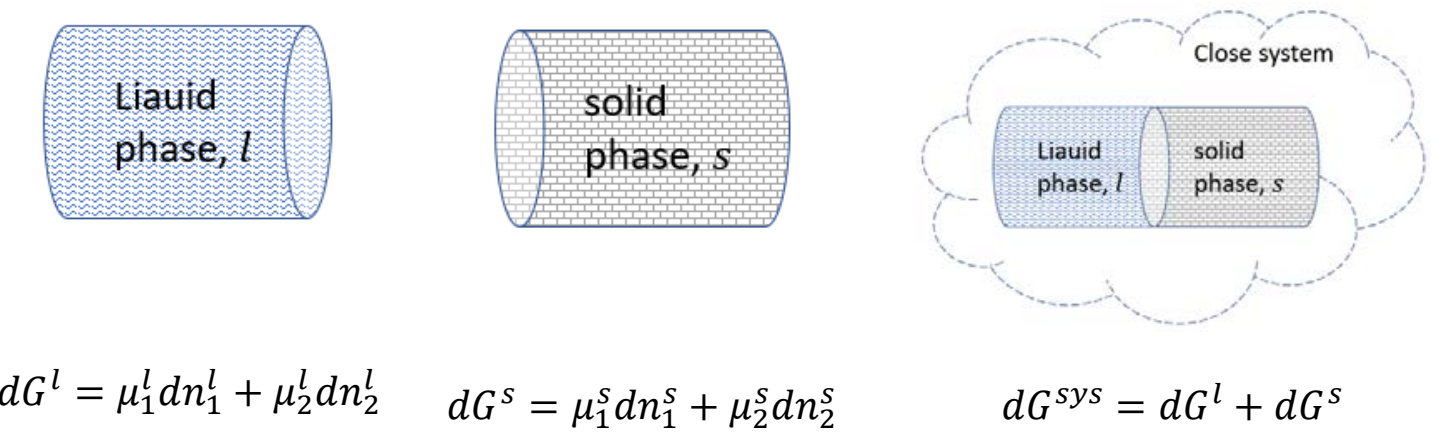

Figure-2.13: The total Gibbs energy of two phases in contact.

If both systems are brought into contact at constant pressure and temperature, the components will transfer between them to reach a new equilibrium state. Since the total system (liquid + solid) is closed, the total amounts of components will remain constant. Thus, an increase of any component in one system is compensated by an equivalent decrease of the component amount in the other phase. Mathematically, this is equal to $d_{n_{A}}^{l}=-d_{n_{A}}^{s}$ and $d_{n_{B}}^{l}=-d_{n_{B}}^{s}$. By substituting these two equations in $d G^{l}$ and $d G^{s}$, the total Gibbs energy at equilibrium is equal to:

$$
d G^{s y s}=d G^{l}+d G^{s}=\left(\mu_{1}^{l}-\mu_{1}^{s}\right) d n_{1}^{l}+\left(\mu_{2}^{l}-\mu_{2}^{s}\right) d n_{2}^{l} \quad E q 2.45
$$


Since at equilibrium $d G^{\text {sys }}=0$, each term $\left(\mu_{1}^{l}-\mu_{1}^{s}\right)$ and $\left(\mu_{2}^{l}-\mu_{2}^{s}\right)$ must also be equal to zero. Eventually, this leads to equality of the chemical potentials at equilibrium: $\mu_{1}^{l}=\mu_{1}^{s}$ and $\mu_{2}^{l}=\mu_{2}^{S}$.

This procedure can be tailored for many phases with many components. An array of chemical potential equalities can be constructed as following:

$$
\begin{aligned}
& \mu_{1}^{1}=\mu_{1}^{2}=\mu_{1}^{3}=\cdots=\mu_{1}^{\emptyset} \\
& \mu_{2}^{1}=\mu_{2}^{2}=\mu_{2}^{3}=\cdots=\mu_{2}^{\emptyset} \\
& \mu_{3}^{1}=\mu_{3}^{2}=\mu_{3}^{3}=\cdots=\mu_{3}^{\emptyset} \quad \text { [chemical potential equality equations] Eq2.46 } \\
& \vdots=\mu_{i}^{2}=\cdots=\mu_{i}^{\varnothing}
\end{aligned}
$$

Each row has $(\varnothing-1)$ equations and since there are $i$ rows, the total numbers of equations is equal to $i \cdot(\varnothing-1)$. Due to mass conservation, not all of the equations are independent. In each column, there are $(i-1)$ independent equations, and $\emptyset \cdot(i-1)$ independent equations in the entire array. Therefore, the number of chemical potential equality equations that describe the equilibrium are equal to

$$
F=\emptyset \cdot(i-1)-i \cdot(\varnothing-1)=i-\emptyset \quad E q 2.47
$$

If $F$ is the degree of freedom and the temperature is another variable, Equation-2.21 yields $F=i-\emptyset+1$, which is the reduced Gibbs phase rule. In addition, there are $\emptyset$ number of equations from the mass conservation in each phase. For example, if a system has of two phases $(s, l)$ and two components $(i=2, \varnothing=2)$, the number of chemical potential equality 
equations is two, $2 \cdot(2-1)$, and the number of mass conservation equations is also two, since $\varnothing=2$. The equations are:

$$
\begin{gathered}
\mu_{1}^{l}=\mu_{1}^{s} \quad \mu_{2}^{l}=\mu_{2}^{s} \\
x_{1}^{l}+x_{2}^{l}=1 \quad x_{1}^{s}+x_{2}^{s}=1
\end{gathered} \quad \text { [Equilibrium equations for a binary system] } E q 2.48
$$

Solving these equilibrium equations defines the boundary between the two phases, and by calculating all the boundaries between the phases, the entire phase diagram can be constructed.

\subsection{Fe-Cr-C Thermodynamics Modeling}

The thermodynamics assessment of the $\mathrm{Fe}-\mathrm{Cr}-\mathrm{C}$ alloy describes phase relations and equilibrium compositions over a wide temperature and composition ranges, and the $\mathrm{Fe}-\mathrm{Cr}$ C system is comprised of many phases according to temperature and composition. The phases are liquid, $\mathrm{BCC}$ ferrite, $\mathrm{FCC}$ austenite, and some carbide phases such as $\mathrm{Cr}_{7} \mathrm{C}_{3}$ and $\mathrm{Cr}_{3} \mathrm{C}_{2}$. An assessment of the $\mathrm{Fe}-\mathrm{Cr}-\mathrm{C}$ system was given by Andersson [40], and it determined that the liquid phase is treated as a substitutional real solution, since it does not have any regular lattices.

$$
\begin{aligned}
G_{m}= & y_{\mathrm{C}}{ }^{\circ} G_{\mathrm{C}}+y_{\mathrm{Cr}}{ }^{\circ} G_{\mathrm{Cr}}+y_{\mathrm{Fe}}{ }^{\circ} G_{\mathrm{Fe}} \\
& +\mathrm{R} T\left(y_{\mathrm{C}} \ln y_{\mathrm{C}}+y_{\mathrm{Cr}} \ln y_{\mathrm{Cr}}+y_{\mathrm{Fe}} \ln y_{\mathrm{Fe}}\right) \\
& +y_{\mathrm{C}} y_{\mathrm{Cr}} L_{\mathrm{C}, \mathrm{Cr}}+y_{\mathrm{C}} y_{\mathrm{Fe}} L_{\mathrm{C}, \mathrm{Fe}}+y_{\mathrm{Cr}} y_{\mathrm{Fe}} L_{\mathrm{Cr}, \mathrm{Fe}} \\
& +y_{\mathrm{C}} y_{\mathrm{Cr}} y_{\mathrm{Fe}}\left(y_{\mathrm{C}}{ }^{1} L_{\mathrm{C}, \mathrm{Cr}, \mathrm{Fe}}+y_{\mathrm{Cr}}{ }^{2} L_{\mathrm{C}, \mathrm{Cr}, \mathrm{Fe}}+y_{\mathrm{Fe}}{ }^{3} L_{\mathrm{C}, \mathrm{Cr}, \mathrm{Fe}}\right)
\end{aligned}
$$


With respect to Equation-2.49, yfe, ycr, yc are the molar fractions of iron, chromium, and carbon atoms, where $\mathrm{R}$ is the gas constant, $\mathrm{T}$ is the temperature, and $L$ is an interaction parameter of the corresponding elements. For example, $L_{C r, C}$ is the interaction parameter of chromium and carbon atoms. It is worth mentioning here that there is no difference between the $L_{C r, C}$ and $L_{C, C r}$ notations. Moreover, all interaction parameters, including $L_{C, F e}, L_{C, C r, F e}^{1}$ are given as functions of temperature. The first line in Equation-2.49 describes the molar Gibbs energy of the mechanical mix, the second line describes the molar Gibbs energy contribution from the configurational entropy, and the third and the fourth lines describe the excess molar Gibbs energy.

Both solid phases (BCC ferrite and FCC austenite) are modeled using sublattice models, since $\mathrm{Fe}$ and $\mathrm{Cr}$ atoms are substitutional elements and carbon atoms are interstitial. Therefore, the notation of $y$ addresses the site fractions rather than the molar fractions. The Gibbs energy of BCC and FCC is given by Equation-2.50.

$$
\begin{aligned}
G_{m}= & y_{\mathrm{Cr}}^{s} y_{\mathrm{Va}}^{t}{ }^{\circ} G_{\mathrm{Cr}: \mathrm{Va}}^{h}+y_{\mathrm{Cr}}^{s} y_{\mathrm{C}}^{t}{ }^{\circ} G_{\mathrm{Cr}: \mathrm{C}}^{h}+y_{\mathrm{Fe}}^{s} y_{\mathrm{Va}}^{t}{ }^{\circ} G_{\mathrm{Fe}: \mathrm{Va}}^{h} \\
& +y_{\mathrm{Fe}}^{s} y_{\mathrm{C}}^{t}{ }^{\circ} G_{\mathrm{Fe}: \mathrm{C}}^{h} \\
& +\mathrm{R} T\left[a\left(y_{\mathrm{Cr}}^{s} \ln y_{\mathrm{Cr}}^{s}+y_{\mathrm{Fe}}^{s} \ln y_{\mathrm{Fe}}^{s}\right)\right. \\
& \left.\quad+c\left(y_{\mathrm{C}}^{t} \ln y_{\mathrm{C}}^{t}+y_{\mathrm{Va}}^{t} \ln y_{\mathrm{Va}}^{t}\right)\right] \\
& +G_{m}^{E}+G_{m}^{m o}
\end{aligned}
$$

where, Va represents the vacancies, and a and c denote the number of sites on each sublattice in a formula unit: $(\mathrm{Fe}, \mathrm{Cr})_{a}(\mathrm{Va}, \mathrm{C})_{b}$. The parameters $a=1, c=3$ are for the BCC phase, and $a=c=1$ are for FCC phase. With respect to Equation-2.50, the first and 
second lines describe the Gibbs energy of the mechanical mix, the third and fourth lines describe the configurational entropy, $G_{m}^{E}$ describes the excess Gibbs energy, and $G_{m}^{m o}$ describes the Gibbs energy of the magnetic effect. There is no magnetic term of Gibbs energy for the FCC phase. With the BCC phase, the free energy due to the magnatic effect $\left(G_{m}^{m o}\right)$ is a function of temperature and compositon, and defined as:

$$
G_{m}^{m o}=R T \ln (\beta+1) f(\tau) \quad[\text { Gibbs energy of magnetic }] \quad \text { Eq2.51 }
$$

The excess molar free energy $\left(G_{m}^{e x}\right)$ for both BCC and FCC is:

$$
\begin{aligned}
G_{m}^{E}= & y_{\mathrm{Cr}}^{s} y_{\mathrm{Fe}}^{s}\left(y_{\mathrm{C}}^{t} L_{\mathrm{Cr}, \mathrm{Fe}: \mathrm{C}}+y_{\mathrm{Va}}^{t} L_{\mathrm{Cr}, \mathrm{Fe}: \mathrm{Va}}\right) \\
& +y_{\mathrm{Va}}^{t} y_{\mathrm{C}}^{t}\left(y_{\mathrm{Cr}}^{s} L_{\mathrm{Cr}: \mathrm{Va}_{,} \mathrm{C}}+y_{\mathrm{Fe}}^{s} L_{\mathrm{Fe}: \mathrm{Va}, \mathrm{C}}\right)
\end{aligned}
$$

where, the interaction parameters $(L)$ are denoted by ' $:$ ' to indicate that the corresponding atoms are in different sublattices. For example, $L_{F e: V a, C}$ indicates the interaction parameter between iron atoms (which are in the first sublattice $s$ ) and vacancies, and the carbon atoms are in the second sublattice $t$. Equations 2.49 and 2.50 define the thermodynamics model of the three phases of Fe-Cr-C alloy in a temperature range of $25-1538^{\circ} \mathrm{C}$. In our solidification model we consider only ferrite $\alpha$ and austenite $\gamma$ phases and do not include carbides.

Another thermodynamics assessment of $\mathrm{Fe}-\mathrm{Cr}-\mathrm{C}$ was proposed by Alexandra et al [41], and they used the same Gibbs energy models for the liquid, BCC, and FCC phases. However, some interaction parameters were modified. For example, a comparison of the 
interaction parameters of the liquid phase of Andersson and Alexandra assessments follows:

Table-2: Interaction parameters of two differnrt Fe-Cr-C thermodynamic model.

\begin{tabular}{|l|l|}
\hline \multicolumn{1}{|c|}{ Andersson's assessment } & \multicolumn{1}{c|}{ Alexandra's assessment } \\
\hline$L_{C r, C}^{0}=-90526-25.9116 T$ & $L_{C r, C}^{0}=-69245-35 T$ \\
\hline$L_{C r, C}^{1}=80000$ & $L_{C r, C}^{1}=83242$ \\
\hline$L_{C r, C}^{2}=80000$ & $L_{C r, C}^{2}=88000$ \\
\hline
\end{tabular}

The important point here is that the difference between two thermodynamics assessments is the definition of interaction parameters as functions of temperature. A thermodynamics computational code is developed in this study to calculate the phase diagram of Fe-Cr-C, as described in the next section. The developed code is used to compare Andersson and Alexandra's thermodynamic assessments, as shown in Figure-2.14.

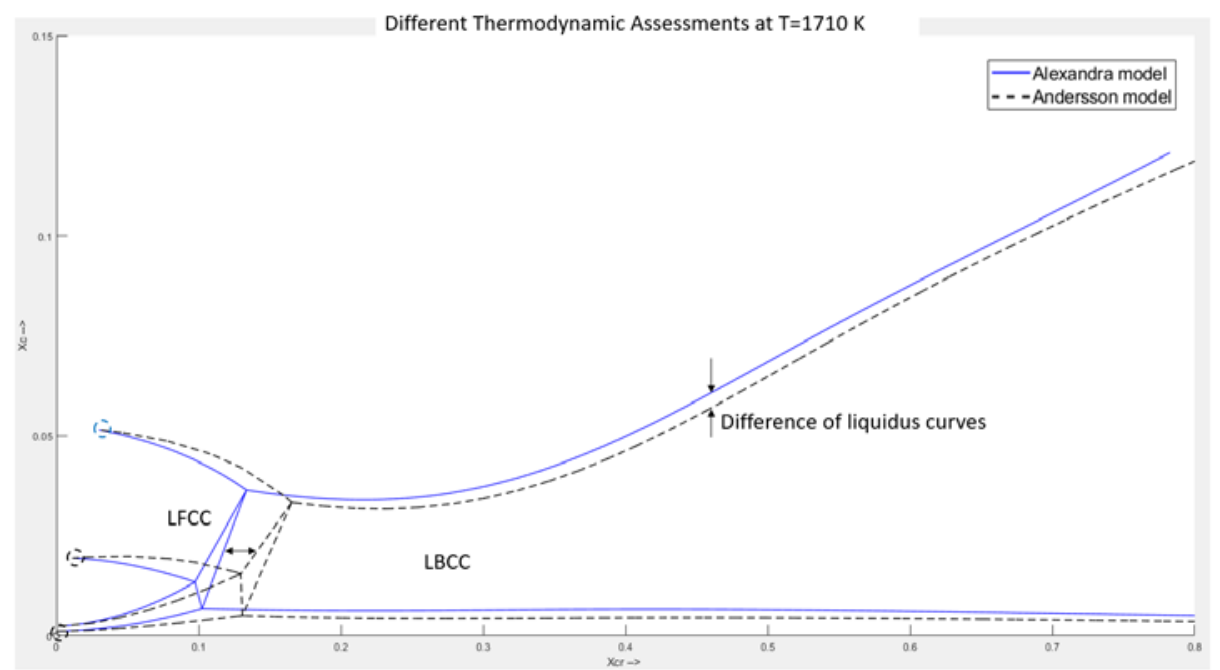

Figure-2.14: A comparesion between Andersson and Alexandra's thermodynamic assessments. 
Figure-2.14 shows that the tie-triangle calculated by Andersson's assessment is shifted to the right that means the stability range of L-FCC zone is extended compared to Alexandra's assessment. The leftmost points (the ones surrounded by the dashed circles) of both assessments are equal that indicates the assessment of the binary $\mathrm{Fe}-\mathrm{C}$ are equal in both models. Finally, the liquidus curve of Andersson's assessment are shifted down which will predict less solid fractions for any thermodynamic state.

Total Gibbs energy comprises all interaction terms that affect phase equilibrium, and the magnetic effect in $\mathrm{BCC}$ is one of the important reaction terms. Though the value of magnetic Gibbs $G^{m a g}$ energy is small at high temperatures, it does affect the stability of the phases. For example, at $\mathrm{T}=1436^{\circ} \mathrm{C}$, the total Gibbs energy of the $\mathrm{BCC}$ phase is equal to $-102,587 \mathrm{~J} / \mathrm{mol}$ and its $G^{m a g}=-68 \mathrm{~J} / \mathrm{mol}$. However, even though, the contribution of $G^{m a g}$ is $0.06 \%$ of the total Gibbs energy of the BCC phase, it affects the phase stability.

\subsection{The Implementation of Fe-Cr-C Thermodynamics Model}

Andersson's thermodynamics assessment is used in this work to compute the data of the Fe-Cr-C phase diagram. In current computations, the range of chromium concentration in alloy is limited so that carbide phases cannot be formed. In addition, the range of temperature at which the liquid phase is in equilibrium with solid phases is 1330 to 1538 ${ }^{\circ} \mathrm{C}$. According to the thermodynamics model used, liquid and $\mathrm{BCC}$ are in equilibrium in a temperature range of 1494 to $1538{ }^{\circ} \mathrm{C}$, and L-BCC-FCC exists in equilibrum within a temperature range of 1330 to $1494{ }^{\circ} \mathrm{C}$. To calculate the phase diagram within these 
temperature ranges, the corresponding system of equations must be solved, and three computational codes have been developed to do this: $L_{-} B C C$ solver, L_FCC solver, and L_BCC_FCC solver. The solvers use the fsolve Matlab command [42].

The first solver resolves the 2.53 set of equations in which liquid and $\mathrm{BCC}$ are in equilibrum. This set of equations has the following eight unknowns: $\left\{x_{F e}^{l}, x_{C r}^{l}, x_{C}^{l}, x_{F e}^{B C C}, x_{C r}^{B C C}, x_{C}^{B C C}, f^{l}, f^{B C C}\right\}$, where $x_{F e}^{l}, x_{C r}^{l}, x_{C}^{l}$ are the molar fraction of iron, chromium, and carbon in liquid phase, and $x_{F e}^{B C C}, x_{C r}^{B C C}, x_{C}^{B C C}$ are the molar fractions of iron, chromium, and carbon in BCC phase. Finally, $f^{l}, f^{B C C}$ are the fraction of liquid and BCC phases.

$$
\begin{array}{cc}
\mu_{f e}^{l}=\mu_{F e}^{B C C} & \\
\mu_{C r}^{l}=\mu_{C r}^{B C C} & {\left[L_{-} B C C \text { solver }\right]} \\
\mu_{C}^{l}=\mu_{C}^{B C C} & \\
x_{F e}^{l}+x_{C r}^{l}+x_{C}^{l}=1 & \\
x_{f e}^{B C C}+x_{C r}^{B C C}+x_{C}^{B C C}=1 & \text { [mass conservation constraints } \quad \text { (Eq2.53) } \\
x_{F e}^{l} \cdot f^{l}+x_{F e}^{B C C} \cdot f^{B C C}=x_{F e}^{0} & \text { [lever rule constraints] } \\
x_{C r}^{l} \cdot f^{l}+x_{C r}^{B C C} \cdot f^{B C C}=x_{C r}^{0} & \\
f^{l}+f^{B C C}=1 &
\end{array}
$$

The second solver resolves the 2.54 set of equations, in which liquid and FCC are in equilibrum. 


$$
\begin{array}{cc}
\mu_{F e}^{l}=\mu_{F e}^{F C C} & \\
\mu_{C r}^{l}=\mu_{C r}^{F C C} & {\left[L_{-} F C C \text { solver }\right]} \\
\mu_{C}^{l}=\mu_{C}^{F C C} & \\
x_{F e}^{l}+x_{C r}^{l}+x_{C}^{l}=1 & \\
x_{F e}^{F C C}+x_{C r}^{F C C}+x_{C}^{F C C}=1 & \text { [mass conservation constraints } \quad \text { (Eq2.54) } \\
x_{F e}^{l} \cdot f^{l}+x_{F e}^{F C C} \cdot f^{F C C}=x_{f e}^{0} & \\
x_{C r}^{l} \cdot f^{l}+x_{C r}^{F C C} \cdot f^{F C C}=x_{C r}^{0} & \\
f^{l}+f^{F C C}=1 &
\end{array}
$$

This set of equations has eight unknowns as follows: $\left\{x_{F e}^{l}, x_{C r}^{l}, x_{C}^{l}, x_{F e}^{F C C}, x_{C r}^{F C C}, x_{C}^{F C C}, f^{l}\right.$, $\left.f^{F C C}\right\}$. The third solver resolves 2.55 set of equations, in which liquid, BCC, and FCC are in equilibrum. This set of equations has twelve unknowns:

$\left\{x_{F e}^{l}, x_{C r}^{l}, x_{C}^{l}, x_{F e}^{B C C}, x_{C r}^{B C C}, x_{C}^{B C C}, x_{F e}^{F C C}, x_{C r}^{F C C}, x_{C}^{F C C}, f^{l}, f^{B C C}, f^{F C C}\right\}$.

The results of each solver define the boundry between the coressponding phases. For example, Figure- 2.15 shows the calculated isotherm at $\mathrm{T}=1514^{\circ} \mathrm{C}$, and the boundary of the mushy zone is calculated based on the $L_{-} B C C$ solver.

$$
\begin{array}{cc}
\mu_{F e}^{l}=\mu_{F e}^{B C C} & \mu_{F e}^{l}=\mu_{F e}^{F C C} \\
\mu_{C r}^{l}=\mu_{C r}^{B C C} & \mu_{C r}^{l}=\mu_{C r}^{F C C} \\
\mu_{C}^{l}=\mu_{C}^{B C C} & \mu_{C}^{l}=\mu_{C}^{F C C} \\
x_{F e}^{l}+x_{C r}^{l}+x_{C}^{l}=1 & \\
x_{F e}^{B C C}+x_{C r}^{B C C}+x_{C}^{B C C}=1 & \\
x_{F e}^{F C C}+x_{C r}^{F C C}+x_{C}^{F C C}=1 & \\
x_{F e}^{l} \cdot f^{l}+x_{F e}^{B C C} \cdot f^{B C C}+x_{F e}^{F C C} \cdot f^{F C C}=x_{F e}^{0} & \text { [mass conservation constraints] } \quad \text { (Eq2.55) } \\
x_{C r}^{l} \cdot f^{l}+x_{C r}^{B C C} \cdot f^{B C C}+x_{F e}^{F C C} \cdot f^{F C C}=x_{C r}^{0} & \\
f^{l}+f^{B C C}+f^{F C C}=1 &
\end{array}
$$




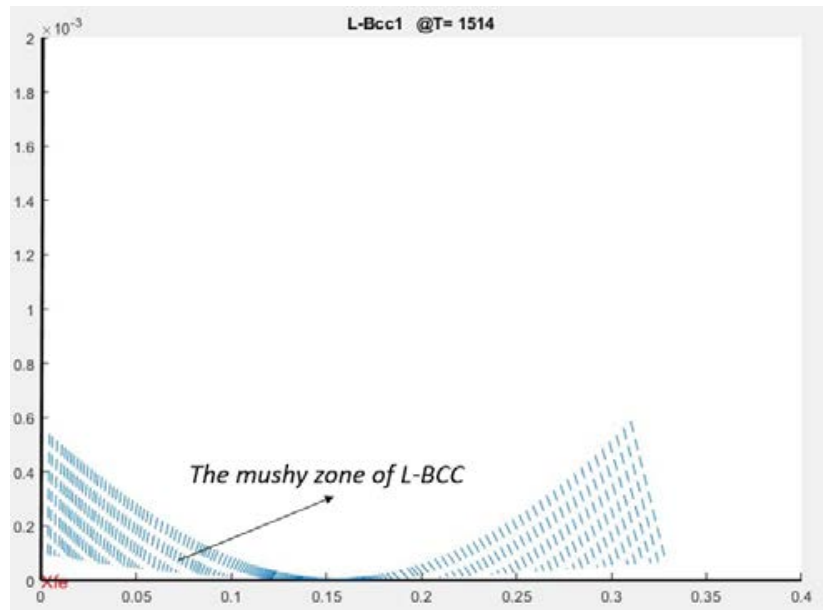

Figure-2.15: The mushy zone of L-BCC as calculated by the L-BCC solver.

Figure-2.16 shows a calculated isotherm at $1437{ }^{\circ} \mathrm{C}$ with three distinct mushy zones and their boundaries. The first mushy zone consists of L-BCC, the second of L-FCC, and the third of L-BCC-FCC. A two phases region consist of BCC-FCC is shown in the figure too. Each zone boundary is calculated by the corresponding solver.

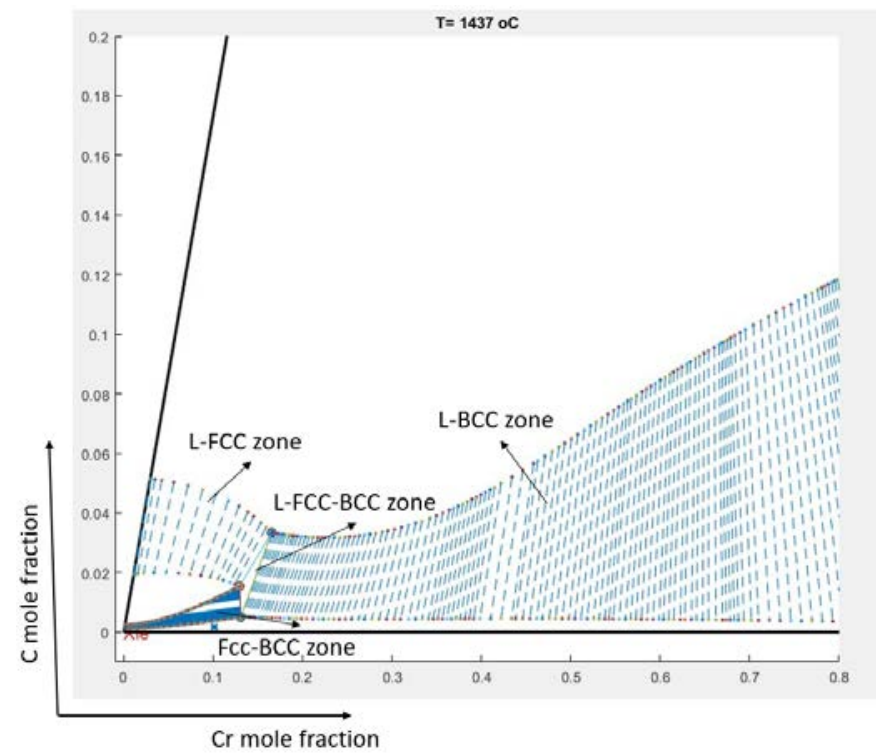

Figure-2.16: Different mushy zones in temperature range two. 


\subsection{Calculation Algorithm and Initial Guess}

For a temperature range of 1330 to $1494{ }^{\circ} \mathrm{C}$ in which L-BCC-FCC is in equilibrum, tietriangles can be calculted as follows. First, the L_FCC_BCC_Solver is provided with an appropriate initial guess and the initial alloy composition $x_{0}$ to find the L-BCC-FCC tietriangle zone. The value of the initial guess is determined by trial and error. It is important that the value of $x_{0}$ is inside or close to the tie-triangle. Once, the triangle is constructed, all other zones can be calculated automatically, as shown in Figure-2.17.

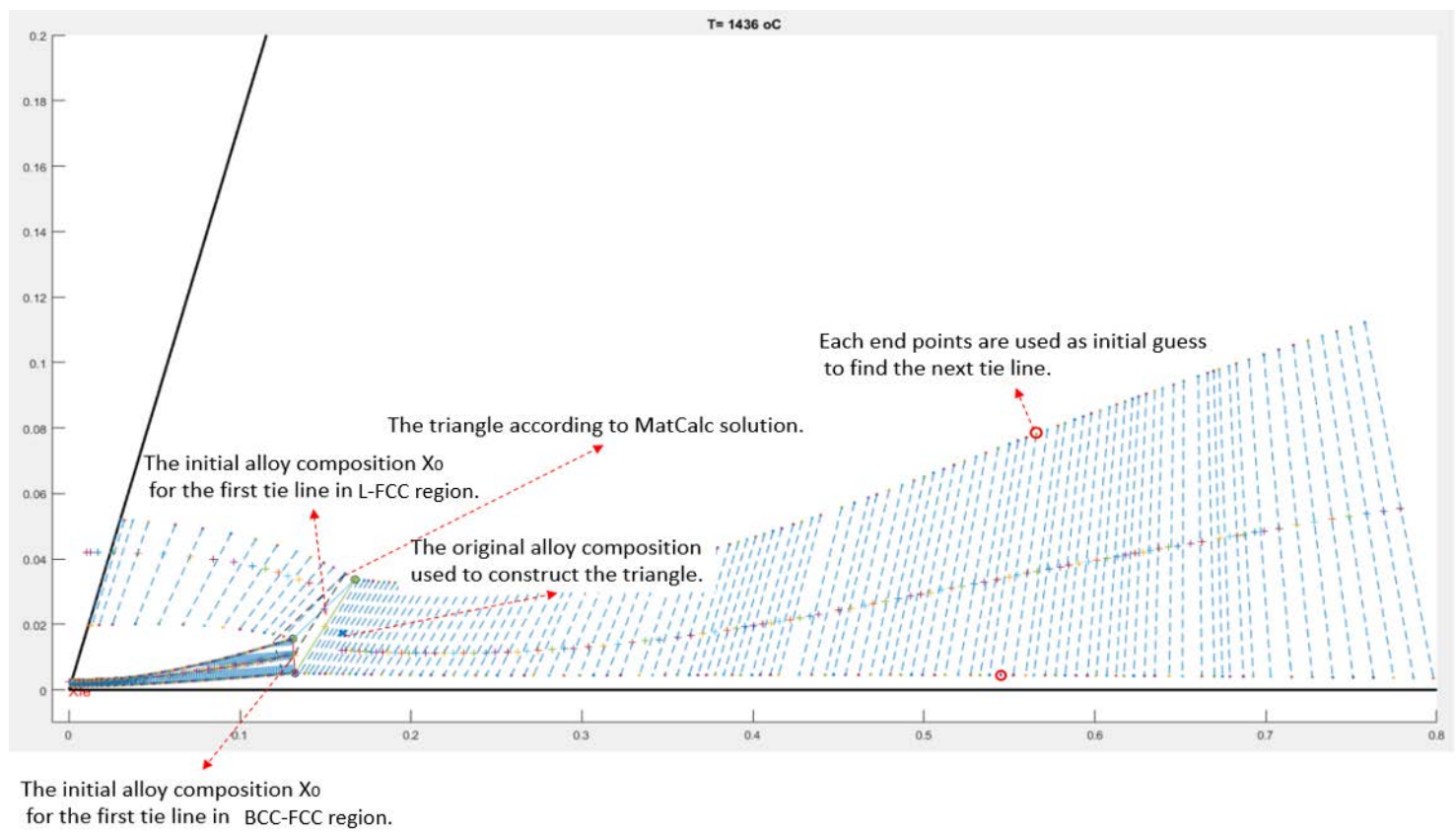

Figure-2.17: The algorithm of the solution.

In Figure-2.17, the midpoint of the left side of the triangle is used as an initial alloy composition to find the first tie line in the L-FCC region. In addition, the tie-triangle vertices at the left are also used as initial guesses. Feeding L_FCC_Solver with a new initial guess and $x_{0}$, yields the first tie line of the L-FCC zone. The second tie line of the 
L-FCC zone is determined in the same way, except that the first tie line is used rather than the triangle side. Every tie line is constructed according to the previous one until the entire L-FCC mushy zone is calculated. The process is repeated for the other two faces of the triangle to calculate the L-BCC and BCC-FCC zones. As stated previously, for this algorithm to work, it needs a suitable initial guess to solve for the tie-triangle in which LFCC-BCC are in equilibrium. Table-3 shows the initial guesses used for different temperature ranges.

Table-3: The used initial guess to calculate the phase diagram.

\begin{tabular}{|c|l|c|}
\hline Temperature Range & \multicolumn{1}{|c|}{ Initial Guess } \\
\hline \multirow{5}{*}{$1502 \leq T \leq 1514^{\circ} \mathrm{C}$} & $x_{F e}^{l}=0.4 \quad x_{C r}^{l}=0.2 \quad x_{C}^{l}=1-x_{F e}^{l}-x_{C r}^{l}$ \\
& $x_{F e}^{B C C}=0.8 \quad x_{C r}^{B C C}=0.11 \quad x_{C}^{B C C}=1-x_{F e}^{B C C}-x_{C r}^{B C C}$ \\
& $x_{F e}^{F C C}=0.8 \quad x_{C r}^{F C C}=0.1 \quad x_{C}^{F C C}=1-x_{F e}^{F C C}-x_{C r}^{F C C}$ \\
& $f^{l}=0.4 \quad f^{B C C}=0.5 \quad f^{F C C}=1-f^{l}-f^{B C C}$ \\
& Initial alloy composition: \\
& $x_{C}^{0}=0.01 \quad x_{C r}^{0}=0.8 \quad x_{F e}^{0}=1-x_{C}^{0}-x_{C r}^{0}$ \\
\hline \multirow{5}{*}{$1494.1 \leq T<1502^{\circ} \mathrm{C}$} & $x_{F e}^{l}=0.75 \quad x_{C r}^{l}=0.2 \quad x_{C}^{l}=1-x_{F e}^{l}-x_{C r}^{l}$ \\
& $x_{F e}^{B C C}=0.8 \quad x_{C r}^{B C C}=0.11 \quad x_{C}^{B C C}=1-x_{F e}^{B C C}-x_{C r}^{B C C}$ \\
& $x_{F e}^{F C C}=0.8 \quad x_{C r}^{F C C}=0.1 \quad x_{C}^{F C C}=1-x_{F e}^{F C C}-x_{C r}^{F C C}$ \\
& $f^{l}=0.4 \quad f^{B C C}=0.5 \quad f^{F C C}=1-f^{l}-f^{B C C}$ \\
& Initial alloy composition: \\
& $x_{C}^{0}=0.01 \quad x_{C r}^{0}=0.11 \quad x_{F e}^{0}=1-x_{C}^{0}-x_{C r}^{0}$ \\
\hline \multirow{5}{*}{$1330 \leq T<1494.1^{\circ} \mathrm{C}$} & $x_{F e}^{l}=0.6 \quad x_{C r}^{l}=0.2 \quad x_{C}^{l}=1-x_{F e}^{l}-x_{C r}^{l}$ \\
& $x_{F e}^{B C C}=0.8 \quad x_{C r}^{B C C}=0.11 \quad x_{C}^{B C C}=1-x_{F e}^{B C C} x_{C r}^{B C C}$ \\
& $x_{F e}^{F C C}=0.8 \quad x_{C r}^{F C C}=0.1 \quad x_{C}^{F C C}=1-x_{F e}^{F C C}-x_{C r}^{F C C}$ \\
& $f^{l}=0.4 \quad f^{B C C}=0.5 \quad f^{F C C}=1-f^{l}-f^{B C C}$ \\
& Initial alloy composition: \\
& $x_{C}^{0}=0.01 \quad x_{C r}^{0}=0.11 \quad x_{F e}^{0}=1-x_{C}^{0}-x_{C r}^{0}$ \\
& & \\
\hline
\end{tabular}




\subsection{The Validation of The CALPHAD Solver}

At every temperature in the range 1330 to $1494^{\circ} \mathrm{C}$, the entire calculation of the $\mathrm{Fe}-\mathrm{Cr}-\mathrm{C}$ phase diagram depends on successful calculation of a tie-triangle zone. In order to verify the developed CALPHAD model, the calculation of the tie-triangle at any temperature is verified against MatCalc ${ }^{\circledR}$. Figure-2.18 shows the result of the verification.

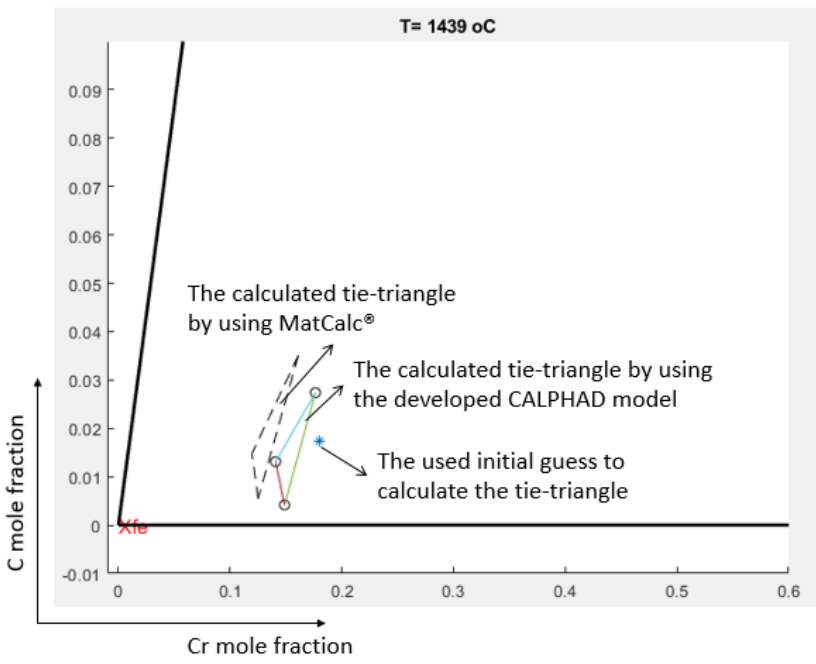

Figure-2.18: The verification of the triangle zone.

The small deviation between the developed model and MatCalc $\AA$ is due to the differences in the thermodynamics database. The developed model is based on Andersson's thermodynamics assessment, and while MatCalc ${ }^{\circledR}$ uses its own assessment. The main difference depends on the interaction parameters of the excess Gibbs energy, such as the difference between the two thermodynamics assessments shown in Figure-2.14. However, the developed CALPHAD model is very flexible and it can allow any updated assessment to be used directly. Figure-2.19a shows an isotherm calculated by Thermo-Calc, and 
Figure-2.19b shows an isotherm calculated by our calculations. Again, the deviation is due to differences in the thermodynamics assessment.
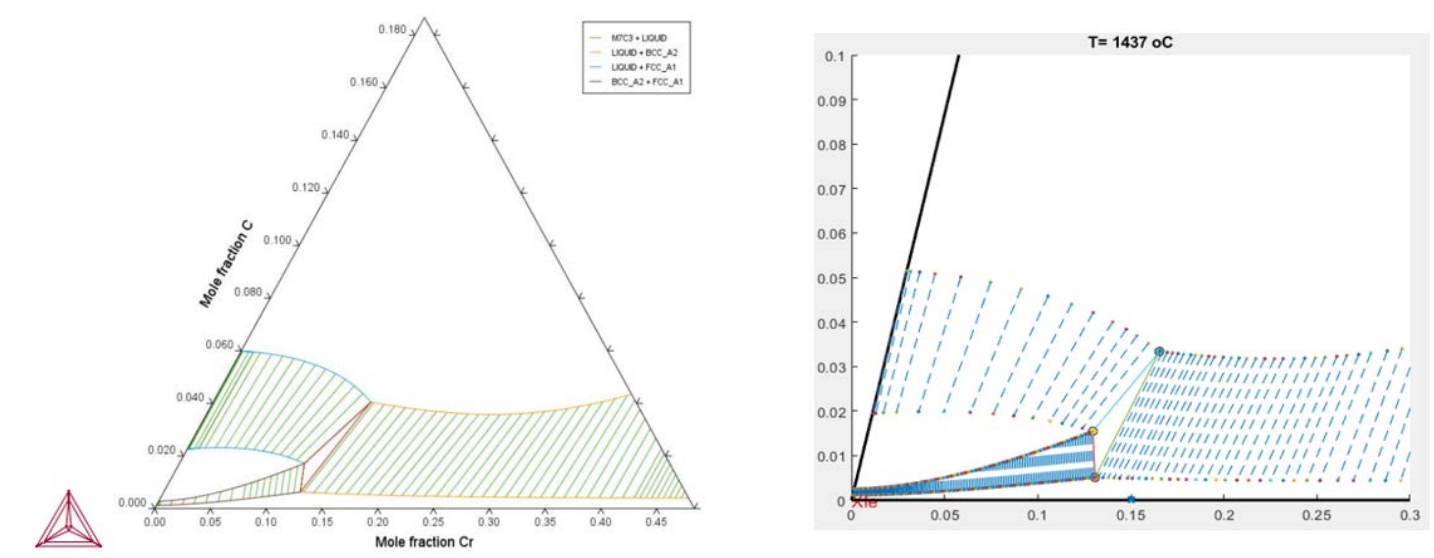

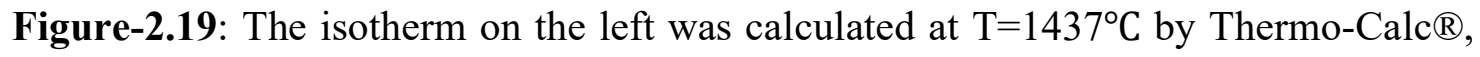
and (b) the isotherm on the right was calculated by the developed model.

Figure-2.20 shows a comparison between two isotherms at $\mathrm{T}=1500{ }^{\circ} \mathrm{C}$ in which liquid and $\mathrm{BCC}$ are in equilibrium.

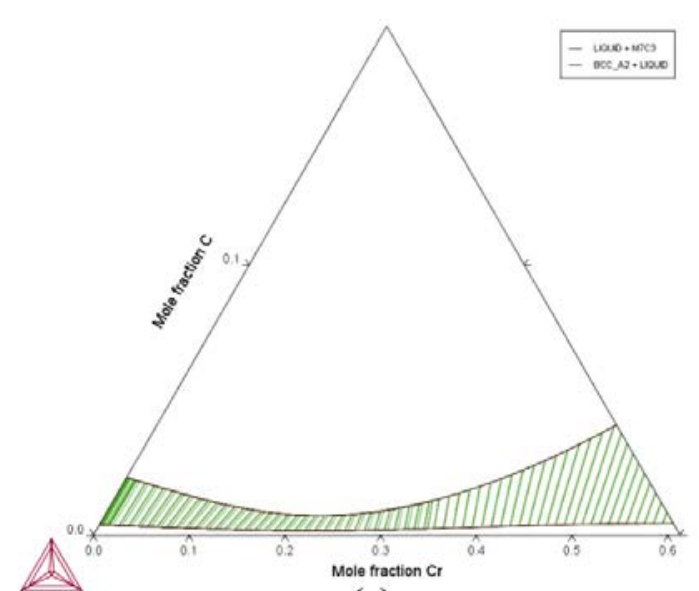

(a)

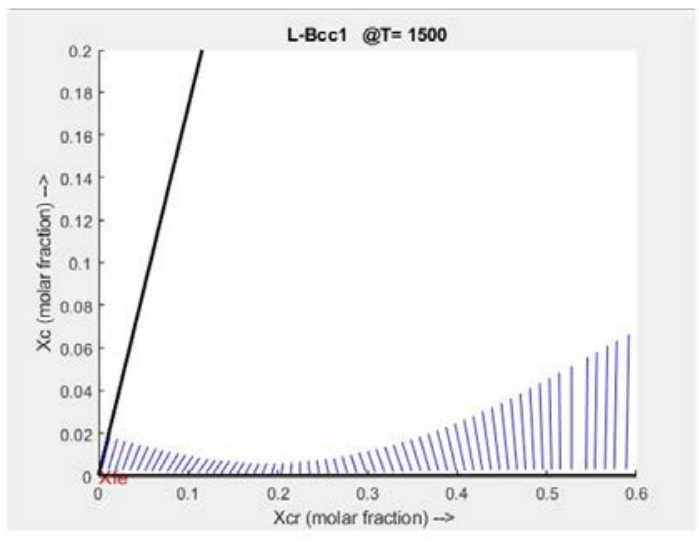

(b)

Figure-2.20: (a) The isotherm at $\mathrm{T}=1500^{\circ} \mathrm{C}$ calculated by Thermo-Calc $\AA$ and (b) the isotherm calculated by the developed model. 
The calculated thermodynamics data by the computational code include: the equilibrium concentration of chromium in liquid $C_{C r}^{l}$, the equilibrium concentration of carbon in liquid $C_{C}^{l}$, the equilibrium concentration of chromium in solid $C_{C r}^{S}$, and the equilibrium concentration of carbon in solid $C_{C}^{S}$. At a constant temperature, the collection of $C_{C r}^{l}$ and $C_{C}^{l}$ describes the liquidus boundary, and the collection of $C_{C r}^{S}$ and $C_{C}^{S}$ describes the solidus boundary. These data are used by the solidification model during the simulation. 


\section{Chapter-3: Solidification Modeling by Cellular Automata CA}

This chapter is composed of two parts: part-A and part-B. Part-A is a broad review of the microstructure solidification modeling by the cellular automata (CA) algorithm. It explains the procedures of computing solute diffusion, heat diffusion, curvature-, and solid fraction, and discusses the effect of mesh anisotropy on solidifying structure. Part-B describes the computational tools developed for solute and heat diffusion, curvature-, and coupling of CALPHAD to CA (or CA-CALPHAD) in detail. Validation of the developed CACALPHAD model is discussed in section-3.9.

\section{Part-A: Using Cellular Automata CA for Soldification Modeling}

\subsection{Cellular Automata (CA)}

The CA algorithm was originally developed by John von Neumann to model complex physical phenomena using simple rules and algorithms [21]. In 1984, the CA algorithm was applied to solidification modeling to simulate the evolution of the microstructure [22]. The procedure of the CA application for solidification modeling consists of four main components: (1) subdivide the computational domain into cells and predetermine the initial state of solid/liquid and boundary conditions for concentration and temperature fields, (2) calculate the state variables (e.g. solid fraction, temperature, solute concentration, curvature) for each cell at every time step, (3) define the neighborhood cells (i.e. first 
neighbor or second neighbor), and (4) specify the transition or capturing rules that determine whether state variables will change their state. The CA algorithm can be used in many materials modeling applications as discussed in many computational material textbooks [38, 43, 44]. This study utilizes the CA algorithm for the simulation of solidification microstructure with varying thermal and compositional fields. According to [23], most published CA solidification models share some common assumptions and features. These include the computational domain in 3D being represented by regular Cartesian grids, and the state variables of cells are temperature, solute concentration, and solid fraction. Additional variables are calculated for interfacial cells (i.e. cells on the $\mathrm{S} / \mathrm{L}$ boundary such as interface curvature and kinetic. Within the computational domain, the

phase state type of any cell can have one of three possible values: solid, liquid, and interface. A solid cell has a solid fraction of one, a liquid cell has a solid fraction of zero, and an interface cell has a solid fraction between zero and one. The S/L interface is implicitly defined in CA by all cells with solid fractions that are between zero and one. In all available models the linearized phase diagram approach was used. In this thesis, more accurate CALPHAD based thermodynamics model is developed.

\subsubsection{Cellular Automata Computational Domain and Cell Neighborhood Definition}

The computational domain characteristics include the size of cells, the total number of cells, and the size of a control volume. Cubic cells are typically considered for a 3D domain, and square cells for a 2D domain. The cell size must be small enough to represent 
the smallest length scale of dendritic morphology (i.e. the tip radius). Using cubic cells produces mesh anisotropy, which is described in the next section. The simplest way to reduce the effect of mesh anisotropy is to use a small cells size in the computational domain. However, this will increase the simulation time significantly because small cell size reduces the time step and increases the total number of cells within a computational domain. To reduce the mesh anisotropy, some models use point mesh or point automata (PA) [45], hexagonal mesh [23], or the mesh refining technique by the quadtree algorithm [46]. In PA, unstructured points are distributed with a computational domain, and initial solid fractions are assigned for each point. In addition, the temperature field is computed for a structured mesh of the domain, and the temperature values of the unstructured points are then interpolated from the structural mesh. In the quadtree refining algorithm the interfacial cells are subdivided into smaller cells, which mitigates the mesh anisotropy effect. However, none of these solutions eliminate the mesh anisotropy completely.

Another important feature of CA is definition of the cell neighborhood. Two types of neighborhoods are widely used: Neumann and Moore. In Neumann neighborhoods, the nearest cells are counted, so there are four cells in a 2D case and six cells in a 3D case. In Moore neighborhoods more cells are counted, so there are eight neighbor cells in a 2D case and 26 cells in 3D case. Figure-3.1 shows these neighborhood definitions. The definition of neighbor cells can be also determined by defining a circle/sphere of a certain radius around a cell, so any cell inside the circle/sphere is a neighbor cell. 

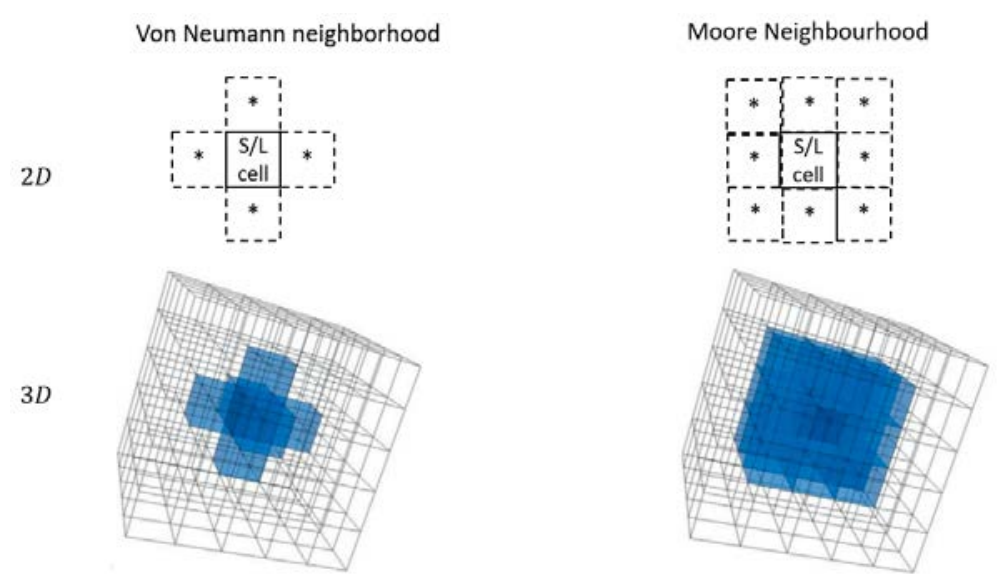

Figure-3.1: The definition of neighborhood of an interfacial cell. [47], page-217.

The neighborhood definition is required for curvature calculations, as well as for calculations of temperature and concentration fields if a finite difference scheme is used with CA, or CA-FD. The calculation of temperature and concentration fields uses the Neumann definition, and the calculation of curvature uses the Moore definition.

\subsubsection{Cellular Automata Transition Rules and Mesh Anisotropy}

All cells in CA computational domain are solid, liquid or interfacial, and some are initially designated as solid or liquid. During CA simulation, solid cells can "capture" liquid cells, and thereby change their state to interfacial. Transition rules dictate this capture process, and the classical transition rule assumes that the state of a cell begins to change if at least one of its Neumann neighbor cells is completely solid. This assumption is reasonable, since a completely solid cell acts as a nucleation site for its Neumann neighbor cells, and once a cell starts to solidify, its solid fraction increases with time. If the transition rule is not applicable for a cell, it will remain liquid with zero solid fraction, even if it has temperature 
is below the liquidus temperature. This transition rule produces strong mesh anisotropy with dendrites growing faster when parallel to the axis of the mesh. Though dendrites can physically grow in every possible direction, strong mesh anisotropy forces dendrites within the computational domain to grow in orthogonal directions. In fact, mesh anisotropy is one of the main disadvantages of using CA in microstructure prediction. However, attempts have been developed to solve the problem, including refining cell size, adjusting the increase of solid fraction, and modifying the transition rules.

In [46], the Quadtree algorithm is used to simulate a thermal dendritic growth and refine the size of interfacial cells locally. This decreases the mesh anisotropy and can predict many dendritic morphologies. Another solution to mesh anisotropy is to direct the increments of solid fractions in different directions than the mesh axis — an adjustment that can be included in the anisotropy function, as shown in [48]. In addition, solid grain growth in different orientations can be achieved by assigning different orientation angles for each solid grain and modifying the capturing rule. In [49] the capturing rule was based on solidifying neighborhood rather than one cell, and if all cells in a defined neighborhood are solid, they will capture another neighborhood cells. The most common modification for capturing rule algorithm is based on the decentered square/octahedron proposed by Rappaz et al [50], and later modified by Wang et al [51]. However, implementing the decentered algorithm requires additional information storage and uses more computational power. Reducing mesh anisotropy and allowing multi-grain orientation growth is more important for casting simulation than welding. This is because a columnar dendritic growth aligned 
with the computational domain axis is for welding simulation, since it reproduces preferred crystallographic growth directions.

\subsubsection{Cellular Automata State Variable Calculations}

The state variables that are typically calculated during CA simulation include the temperature field, the solute concentration field, S/L interface curvature-, and the solid fractions of interfacial cells. The temperature field can either be imposed on a computational domain or calculated. An imposed temperature field assigns uniform values or gradient undercooled temperatures for all cells, which allows a predetermined cooling rate value to be applied at every time step for all cells. The imposed temperature field is reasonable, since the heat diffusion process is more than an order of magnitude faster than the solute diffusion process (the release of latent heat is not considered in this case). However, the temperature field for the entire domain can be solved numerically using an implicit heat equation solver at the same time step as the explicit solute diffusion solver [52]. Some models $[53,54]$ solve the heat equation on a macroscale, then interpolate the temperature values for the $\mathrm{CA}$ microscale domain.

Due to discontinuity at the $\mathrm{S} / \mathrm{L}$ interface, calculation of the solute field is more difficult than calculating the temperature field. Two approaches are proposed to address this: onedomain and two-domain. In the one-domain approach, the diffusion equation is solved over the entire domain for either liquid or solid, and then a correction is considered for the other

phase. For example, Dilthey [23] proposed a one-domain approach that solves the solute 
concentration of the solid phase for the entire domain and gives the solute concentration of liquid by dividing the solid solute concentration by a constant distribution coefficient. In the two-domain approach the solute diffusion equation is solved in the liquid and solid domains separately. The solute field in the interface cells is calculated from the flux from both the solid liquid.

The evolution of the solid fraction $f^{s}$ is incorporated by considering the $\mathrm{S} / \mathrm{L}$ interface velocity or the $\mathrm{S} / \mathrm{L}$ interface kinetic $[23,3]$. The conservation of mass allows calculation of the velocity of the $\mathrm{S} / \mathrm{L}$ interface from the surrounding concentration field, and the change of the solid fraction can be calculated from the velocity of the advanced S/L interface.

However, calculating the solid fraction from the interface velocity requires consideration of geometrical assumptions for the interface inside the cell, one of which could be that the $\mathrm{S} / \mathrm{L}$ interface inside a cell is advancing both vertically and horizontally, as illustrated Figure-3.2a. Thus, the velocity components must be calculated according to the solute concentration of the surrounding cells, and the solid fraction of the cell is defined as the area behind the advanced $\mathrm{S} / \mathrm{L}$ interface in both directions.
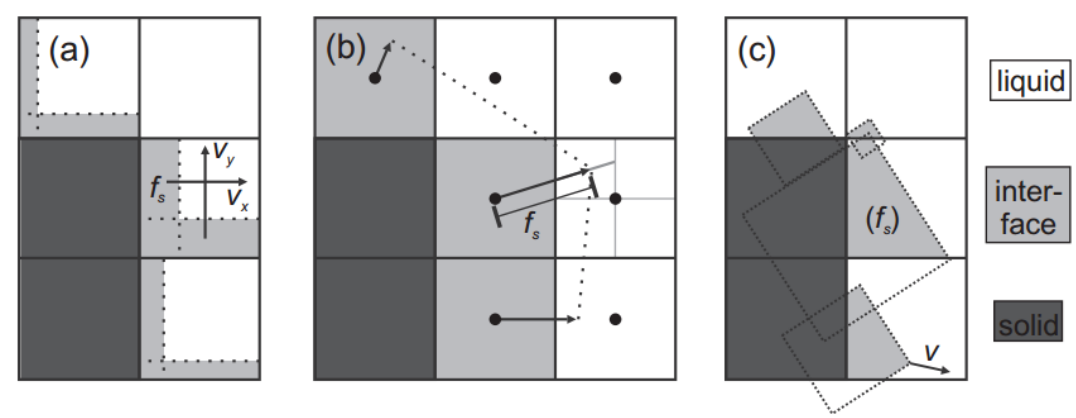

Figure-3.2: Geometrical assumptions of the moving $\mathrm{S} / \mathrm{L}$ interface inside a cell. 
Another geometrical assumption is that the advance of the $\mathrm{S} / \mathrm{L}$ interface is in the direction of the interface normal vector, and the solid fraction is equal to the area covered by a line perpendicular to the normal interface, as shown in Figure-3.2b. The most common interface geometrical assumption is decentered squares, as shown in Figure-3.2c. All the previous assumptions presume that the concentration of solute at the interface is equal to the equilibrium concentration. However, these become invalid as the velocity of the interface increases.

The solid fraction can also be calculated using the kinetics equation of solidification, in which the velocity of the S/L interface movement $\left(V^{s / l}\right)$ is equal to the kinetic coefficient $\left(\mu_{k}\right)$ multiplied by the total undercooling. Total undercooling includes melt undercooling, constitutional undercooling and capillarity undercooling, according to the curvature from Equation-3.1.

$$
V^{s / l}=\mu_{k} \cdot\left[\left(T_{m}^{e}-T_{l i q}\right)+m \cdot C+(\Gamma \cdot k)\right] \quad E q 3.1
$$

where $\left(T_{m}^{e}\right)$ is the melting temperature of the host component, $\left(T_{l i q}\right)$ is the temperature of the liquid, $m$ is the slope of the liquidus line, $C$ is the solute concentration, and $(k)$ is the curvature value (which is negative if convex and positive if concave). Thus, the solid fraction $\left(\Delta f_{S}\right)$ for a cubic cell can be calculated as:

$$
\Delta f_{s}=\frac{\Delta t \cdot V^{s / l}}{\Delta x} \quad E q 3.2
$$


where $\Delta t$ is the time step and $\Delta x$ is the length of the cell. Another method for calculating the solid fraction is the cellwise mass balance, in which the solid fractions of interface cells are calculated by balancing the solute concentration of interface cells with the equilibrium concentration using the lever rule. This approach does not require computation of the velocity of the advancing $\mathrm{S} / \mathrm{L}$ interface, and it gives better mass conservation.

\subsubsection{The Computation of S/L Interface Curvature}

During dendrite growth simulation, the effect of the $\mathrm{S} / \mathrm{L}$ interface curvature on the melting temperature should be included. The computational domain of the simulation is composed of cubic cells, and there could be problem using cubic cells to illustrate the very complex and curved geometry of a dendrite. The computed curvature $\left(K_{r}\right)$ should not be overestimated nor underestimated. If it is overestimated, the resulting dendritic morphology will be blunter than it should be, and if $K_{r}$ is underestimated, the resulting dendritic morphology will be sharper with spike-like branches, as in Figure-3.3.

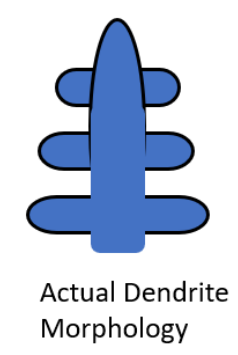

(a)

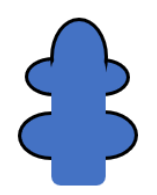

Dendrite morphology with overestimated curvature

More columnar structure.

Shorter/blunter/less branches.

(b)

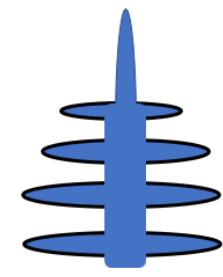

Dendrite morphology with underestimated curvature

More dendritic structure. longer/sharper/more branches.

(c)

Figure-3.3: (a) a dendrite with actual curvature, (b) the dendrite with overestimated curvature, and (c) the dendrite with underestimated curvature. 
Two methods are used to calculate the curvature of the $\mathrm{S} / \mathrm{L}$ interface in $\mathrm{CA}$ : cell count and level set. In the cell count method, the number of cells below a planar front $\left(N_{o}\right)$ and the number of solid cells is counted $\left(N_{S}\right)$. As shown in Figure-3.4, if $N_{o}>N_{S}$ the $\mathrm{S} / \mathrm{L}$ interface is convex, if $N_{o}<N_{s}$ the $\mathrm{S} / \mathrm{L}$ interface is concave; and it is flat if $N_{o}=N_{s}$. There are many empirical formulas used in this method, and the most common was proposed by Nastac [52].

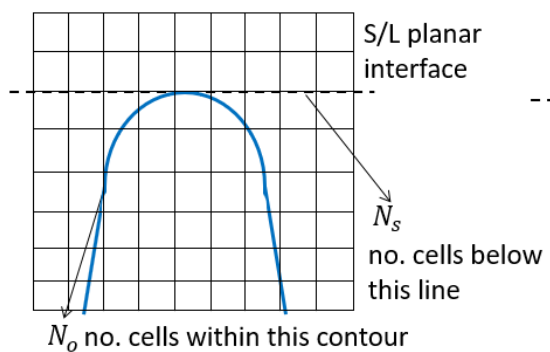

(a) $N_{s}>N_{o}$

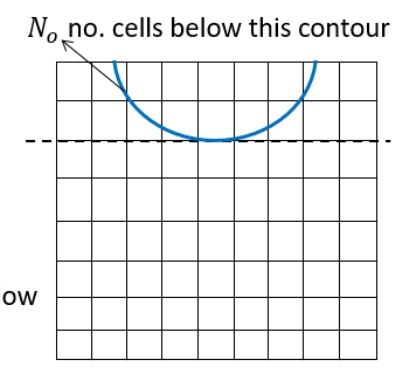

(b) $N_{o}>N_{s}$

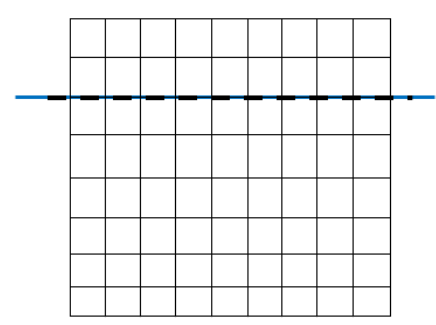

(c) $N_{s}=N_{o}$

Figure-3.4: Calculating curvatures by cell count method.

Level set is another technique to estimate the curvature of an $\mathrm{S} / \mathrm{L}$ interface in CA. With this method, the curvature at any point is the gradient of the unit normal vector of the surface at that point. Since the surface is represented by the solid fraction values in CA, the curvature of an interfacial cell is calculated based on the value of its solid fraction. Thus, the mean curvature $k$ can be calculated as:

$$
K_{r}=\nabla \cdot \frac{\nabla f^{s}}{\left|\nabla f^{s}\right|} \quad E q 3.3
$$

And the expansion of Equation-3.3 is: 


$$
\begin{aligned}
& f_{x}^{s^{2}}\left(f_{y y}^{s}+f_{z z}^{s}\right)+f_{y}^{s^{2}}\left(f_{x x}^{s}+f_{z z}^{s}\right)+f_{z}^{s^{2}}\left(f_{x x}^{s}+f_{y y}^{s}\right)- \\
& K_{r}=\frac{2\left(f_{x}^{s} f_{y}^{s} f_{x y}^{s}+f_{x}^{s} f_{z}^{s} f_{x z}^{s}+f_{y}^{s} f_{z}^{s} f_{y z}^{s}\right)}{\left(f_{x}^{s^{2}}+f_{y}^{s^{2}}+f_{z}^{s^{2}}\right)^{3 / 2}} \quad \text { [level set curvature] } \quad E q 3.4
\end{aligned}
$$

All derivatives in Equation-3.4 can be calculated by using finite difference scheme for the solid fraction field. For example, the derivatives in the x-direction of the solid fraction field evaluated at cell id $\mathrm{i}, \mathrm{j}, \mathrm{k}$ are

$$
\begin{aligned}
f_{x}^{s} & =\frac{f_{i+1 j k}^{s}-f_{i-1 j k}^{s}}{2 \cdot \Delta x} \\
f_{x x}^{s} & =\frac{f_{i+1 j k}^{s}-2 f_{i-1 j k}^{s}+f_{i-1 j k}^{s}}{\Delta x^{2}} \\
f_{x y}^{s} & =\frac{f_{i+1 j+1 k}^{s}-f_{i-1 j+1 k}^{s}-f_{i+1 j-1 k}^{s}+f_{i-1 j-1 k}^{s}}{4 \cdot \Delta x^{4}}
\end{aligned}
$$

The anisotropy of the surface energy should be incorporated with the curvature calculation into the capillarity undercooling calculation using Gibbs-Thomson-Herring relationship as described in 1.5.5. The used model in the current work follows:

$$
\begin{gathered}
d T_{r}=\Gamma \cdot \gamma\left(\theta_{x y}, \theta_{z}\right) \cdot K_{r} \quad E q 3.6 \\
\gamma\left(\theta_{x y}, \theta_{z}\right)=1-\varepsilon_{x y} \cos \left(4 \theta_{x y}\right)-\varepsilon_{z} \cos \left(4 \theta_{z}\right) \quad E q 3.7
\end{gathered}
$$

where, $\varepsilon_{x y}$ is the anisotropy coefficient in the xy plane, $\varepsilon_{z}$ is the anisotropy coefficient in the z-direction, $\theta_{z}=\cos ^{-1}\left(n_{z}\right), \theta_{x y}=\cos ^{-1}\left(n_{x} / \sqrt{n_{x}^{2}+n_{y}^{2}}\right)$ or $\theta_{x y}=\cos ^{-1}\left(n_{x}\right)$ if $n_{x}$ and $n_{y}$ are equal to zero. The normal unit vectors $\left(n_{x}, n_{y}, n_{z}\right)$ are calculated using the derivatives of the solid fraction field as: 


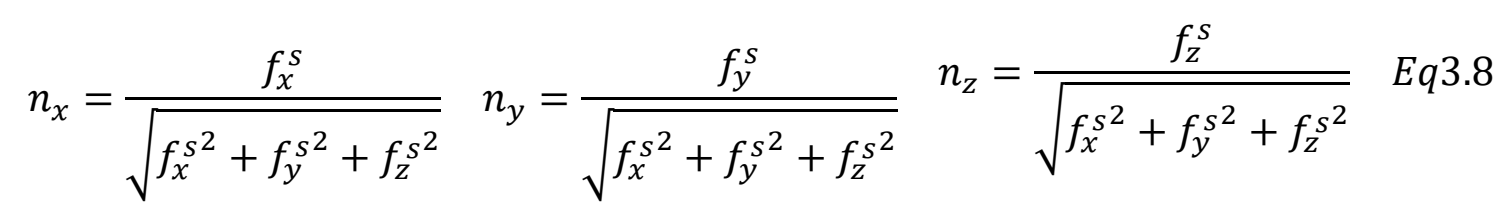

\subsection{Overview of Some Cellular Automata Models}

The varieties of CA models used for solidification modeling are vast as discussed next. Several questions can be asked to distinguish different types of CA models.

- Does the model consider constrained growth, free growth, or both? Since the growth is constrained by the temperature field, using free growth means heat flows from the solid phase into the surrounding and undercooled liquid (this is common in equiaxed grain growth). With constrained growth, heat flows from the surrounding liquid into the solid, which is common in columnar/dendritic growth. If a correct nucleation algorithm is used, the model can predict columnar-to-equiaxed growth transition [13].

- Does the model solve for thermal dendrites, solutal dendrites, or thermo-solutal dendrites? With thermal dendrites only the heat equation is solved, so they are typically used for the solidification of pure materials, and the solution scheme can be explicit or implicit. The boundary conditions can be natural convection or the Neumann type. With solutal dendrites the mass diffusion equation is solved and an imposed temperature field with some undercooling can be assumed. In thermo-solutal dendrites both heat diffusion and mass diffusion equations are solved at each time step. 
- How does the model determine the curvature? The most common two methods for this are cell-count and level-set?

- How does the model include the physical anisotropy of the growth, and manage mesh anisotropy?

- How does the model estimate the kinetics of the $\mathrm{S} / \mathrm{L}$ interface? The kinetics of the interface is included by calculating the increments in solid fraction at each time step. The increments can be based on the Stefan condition or kinetic undercooling.

Next, some noteworthy models are analyzed by answering the previous questions.

Gandin and Rappaz used the CA algorithm to simulate the microstructure of a casting, and predicted a meso-scale dendrite envelope [23]. In [55], the model was coupled with finite elements ( CA-FE) to compute the temperature field. The model was later extented to a 3D case [56] by proposing 3D decentred octahedron CA growth count for different grain orinetations. In [57], the model was used to simulate casting of aluminum-silicon rods.

Sasikumar et al [58] proposed a 2D CA model for pure metals that solved the heat equation explicitly. The solid fraction was calculated using the kinetics of the interface using the stefan condition in $\mathrm{x}$ and $\mathrm{y}$ directions. The model was used to study the effects of undercooling, surface tension, and solidification noise on the solidification. In [59], Sasikumar et al used the developed model to study the effect of the Gibbs-Thomson coefficient $(\Gamma)$ on grain coarsening, and concluded that a higher $\Gamma$ produces an artificial Ostwald ripening phenomenon that is equvelant to the simulation running for a long time. 
V. Pavlyk and U. Dilthey [60] developed a 2D cellular automata model to simulate the crystal growth of a binary alloy. They considered a mass balance with Stefan-like conditions at the S/L interface, and solved the mass difusion equation explicitly at the S/L interface to account for solid fraction incrementation. They also coupled the model with finite difference to simulate dendritic growth in a weld pool [44].

Nastac developed a 2D CA model [61], then extended it to 3D [52]. His main new contribution was solving the heat equation implicitly to calculate the temperature field during simulation. His second contribution was developing the cell-count method to calculate the curvature of a cell by accounting for the solid fractions within its Moore neighborhood.

\section{Part-B: The CA Model of the Current Work}

\subsection{Computational Tools}

As described, the implementation of CA algorithms requires calculation of certain field variables. Four computational tools are developed in this study to compute: the solute field,

the temperature field, the curvature field, and the solid fraction. The calculation of solid fraction is based on the equilibrium information of the phase diagram obtained by CALPHAD calculations. These tools calculate the fields at each time step. 


\subsubsection{Mass Transport Computation Tool}

The solute distribution can be determined by solving the mass diffusion equation by the finite volume FV method as follows:

$$
\begin{aligned}
\frac{\partial C}{\partial t}=D^{l}(T) \cdot\left(\frac{\partial^{2} C}{\partial x^{2}}+\frac{\partial^{2} C}{\partial y^{2}}+\frac{\partial^{2} C}{\partial z^{2}}\right) \quad\left[\text { Fick's second law of diffusion] }^{\prime} \quad\right. \text { Eq3.9 } \\
\qquad\left[\begin{array}{l}
\left(\frac{C_{i+1, j, k}^{t}-2 \cdot C_{i, j, k}^{t}+C_{i-1, j, k}^{t}}{\Delta x^{2}}\right)+ \\
C_{i j k}^{t+1}-C_{i j k}^{t}=\Delta t \cdot D^{l}(T) \cdot\left[\frac{C_{i, j+1, k}^{t}-2 \cdot C_{i, j, k}^{t}+C_{i, j-1, k}^{t}}{\Delta y^{2}}\right)+ \\
\left(\frac{C_{i, j, k+1}^{t}-2 \cdot C_{i, j, k}^{t}+C_{i, j, k-1}^{t}}{\Delta z^{2}}\right)
\end{array}\right] \quad E q 3.10
\end{aligned}
$$

Our model assumes that: (1) the diffusion coefficient is independent of temperature, (2) there is no diffusion in the solid phase, and (3) there is no back diffusion from liquid to solid - all these assumptions should be included in the FV formulation. The first assumption can be incorporated by using a constant value for the diffusion coefficient for each species. The second assumption is incorporated by solving the FV formulation for liquid and interfacial cells only. This assumption is reasonable since the diffusivity of solutes in solid is hundreds of time smaller than that is in liquid.

Another important consideration is diffusion through interfacial cells, since they are partially liquid and partially solid. Diffusion only occurs in the liquid portion, and this should be considered in the formulation. An interfacial cell and its neighbor cells are depicted in Figure-3.5. The flux of solutes into the interfacial cell from its liquid neighbor 
cells occurs in three directions $(\mathrm{x}, \mathrm{y}, \mathrm{z})$. Only flux in the $\mathrm{x}$-direction is considered here, to show how an interfacial cell can be treated.

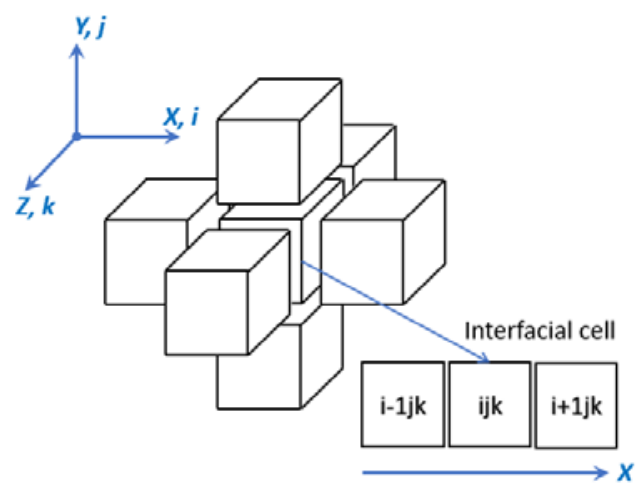

Figure-3.5: Finite difference formulation of an interfacial cell.

The change in interfacial cell concentration is equal to the change in its mass divided by its volume. However, the cell volume must be multiplied by its liquid fraction, since the diffusion occurs in liquid only:

$$
\Delta C=\frac{\Delta M}{V \cdot f^{l}} \quad[\text { the concentration of an interfacial cell }] \quad E q 3.11
$$

The change in the cell mass along the $\mathrm{x}$-direction is equal to the net mass flux multiplied by the flux area and the time step:

$$
\Delta M=\operatorname{flux}_{n e t} \cdot \Delta y \cdot \Delta z \cdot d t \quad[\text { mass change }] \quad \text { Eq3.12 }
$$

By substituting Equation-5 into Equation-4, the change in concentration of the interfacial cell is:

$$
\Delta C=\frac{f l u x_{n e t} \cdot \Delta y \cdot \Delta z \cdot d t}{\Delta x \cdot \Delta y \cdot \Delta z \cdot f^{l}}
$$


and the net flux into the cell is obtained by FD formulation of the diffusion equation:

$$
f_{\text {lux }}=\operatorname{flux}_{\text {out }}-\operatorname{flux}_{\text {in }}=D^{l} \cdot\left(\frac{C_{i+1}-C_{i}}{\Delta x}\right)-D^{l} \cdot\left(\frac{C_{i}-C_{i-1}}{\Delta x}\right)
$$

The concentration changes in the cell due to solute flux in the $\mathrm{x}$ direction are obtained by substituting Equation-3.14 into Equation-3.13:

$$
\Delta C=\frac{D^{l} \cdot \Delta t}{f^{l}} \cdot\left(\frac{C_{i+1}-C_{i}}{\Delta x^{2}}-\frac{C_{i}-C_{i-1}}{\Delta x^{2}}\right) \quad E q 3.15
$$

Since the same diffusion process also occurs in the $y$ and $z$ directions, Equation-3.15 can be rewritten in term of $\mathrm{y}$ and $\mathrm{z}$ directions. A special modification of Equation-3.10 is required to incorporate the third assumption: a diffusion block function, $\theta\left(f^{s}\right)$, is defined as:

$$
\theta\left(f^{s}\right)=\left\{\begin{array}{ll}
0 & \text { if } f^{s}=1 \\
1 & \text { if } f^{s}<1
\end{array} \quad[\text { Diffusion block function] } \quad \text { Eq3.16 }\right.
$$

As the value of the blocking function can be either one or zero, it blocks diffusion from or to cells that are completely solid. Eventually, the full FV formulation for solute diffusion is:

$$
C_{i j k}^{l+1}=C_{i j k}^{l}+\frac{D^{l} \cdot \Delta t}{f_{i j k}^{l}} \cdot\left[\begin{array}{l}
\frac{\left(C_{i+1, j, k}^{l}-C_{i j k}^{l}\right) \cdot \theta\left(f^{s}\right)}{\Delta x^{2}}-\frac{\left(C_{i, j, k}^{l}-C_{i-1, j, k}^{l}\right) \cdot \theta\left(f^{s}\right)}{\Delta x^{2}}+ \\
\frac{\left(C_{i, j+1, k}^{l}-C_{i j k}^{l}\right) \cdot \theta\left(f^{s}\right)}{\Delta y^{2}}-\frac{\left(C_{i, j, k}^{l}-C_{i, j-1, k}^{l}\right) \cdot \theta\left(f^{s}\right)}{\Delta y^{2}}+ \\
\frac{\left(C_{i, j+1}^{l}-C_{i j k}^{l}\right) \cdot \theta\left(f^{s}\right)}{\Delta z^{2}}-\frac{\left(C_{i, j, k}^{l}-C_{i, j, k-1}^{l}\right) \cdot \theta\left(f^{s}\right)}{\Delta z^{2}}
\end{array}\right] \quad[\mathrm{FV} \text { formulation }] \quad E q 3.17
$$


The time step is selected based on the Fourier stability criteria of the explicit scheme: $\Delta t \leq$ $\frac{\Delta x^{2}}{8 \alpha}$, where $\Delta x$ and $\alpha$ are the cell size and the solute diffusivity respectively. Based on Equation-3.17, the solute transportation is computed with the following algorithm. The control volume of the solidification model is first subdivided into three domains: the cells in the first domain are completely liquid $\Omega^{l}$, those in the second domain are completely solid $\Omega^{S}$, and those in the third domain are in mushy state $\Omega^{s / l}$. Initially, all cells in the bottom layer of the control volume are completely solid, and the adjacent cells are in mushy state. The rest of the cells are in liquid state, as shown in Figure-3.6.

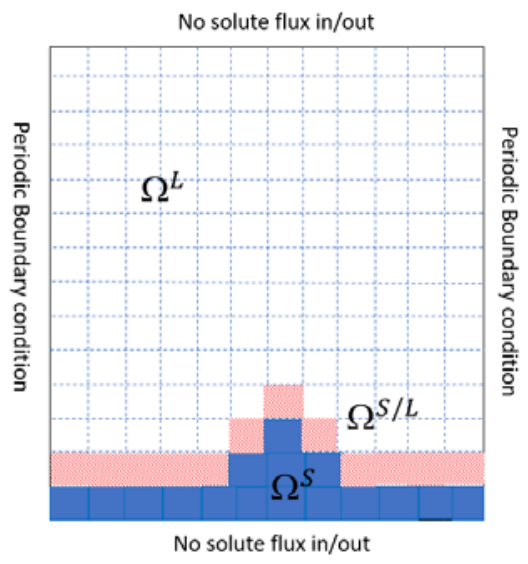

Figure-3.6: The computational domain of mass diffusion computation.

In order to conserve the mass, periodical boundary conditions are applied in the $\mathrm{x}$ and $\mathrm{y}$ directions, and no flux moves in or out in the $\mathrm{z}$ direction. The concentration of the liquid domain cells is initially set to a constant value equal to the initial alloy composition, $C_{0}$, and the concentration of the interfacial cells is set to the equilibrium concentration, $C_{e q}$. Therefore, the concentration gradient will drive solute transport through multiple step. 


\subsubsection{Imposed Temperature Field Computation}

The temperature field can be imposed on a computational domain, in which initial temperature values are assigned for all cells. The initial temperature values can be uniform or with gradient, as shown in Figure-3.7. In either case, a constant cooling rate $(\mathrm{K} / \mathrm{s})$ can be applied on the computational domain. Such approach is used in $[62,3]$.
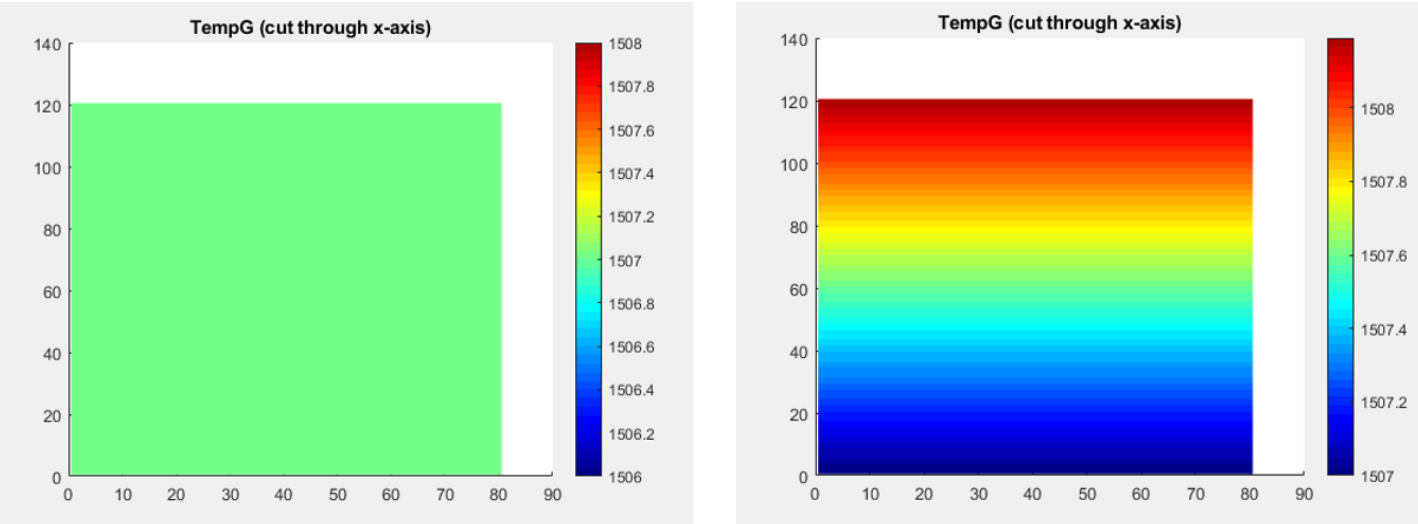

(a) uniform imposed temperature $1507^{\circ} \mathrm{C}$

(b) imposed temperature with gradient

Figure-3.7: Imposed temperature profile.

On the other hand, the temperature field can be imported from a macroscopic FE mesh of a welding case, and the procedure is described in chapter 5 .

\subsubsection{Heat Transport Computation}

The heat diffusion equation can also be calculated for a computational domain to determine the temperature field. The enthalpy formulation of heat equation is implemented in this study. With this method, the differential equation of heat transport should be formulated to 
include the enthalpy, which is the total heat content of the substance. In Equation-3.18, the enthalpy function is used as a dependent variable with the temperature.

$\frac{\partial H}{\partial t}=k_{t h} \cdot\left[\left(\frac{\partial^{2} T}{\partial x^{2}}\right)+\left(\frac{\partial^{2} T}{\partial y^{2}}\right)+\left(\frac{\partial^{2} T}{\partial z^{2}}\right)\right] \quad[$ heat transfer with phase - change $]$

where, $k_{t h}$ is the thermal conductivity. For a numerical scheme, the temperature of the entire domain is specified at each time step, and the enthalpy is solved. The temperature for the next time step is then calculated from the enthalpy. In interfacial cells, additional phase equilibrium condition should be considered as described in section-3.3.5. Writing Equation-3.18 in finite volume scheme gives:

$\frac{\partial H}{\partial t} \approx \frac{H_{i j k}^{t+1}-H_{i j k}^{t}}{\Delta t}=k_{t h} \cdot\left[\begin{array}{c}\left(\frac{T_{i+1 j k}-2 T_{i j k}+T_{i-1 j k}}{\Delta x^{2}}\right)+\left(\frac{T_{i j+1 k}-2 T_{i j k}+T_{i j-1 k}}{\Delta y^{2}}\right) \\ +\left(\frac{T_{i j k+1}-2 T_{i j k}+T_{i j k-1}}{\Delta z^{2}}\right)\end{array}\right]$

For a cubic cell $\Delta x=\Delta y=\Delta z$ so

$d H=H_{i j k}^{t+1}-H_{i j k}^{t}=\Delta t \cdot k_{t h} \cdot\left[\begin{array}{c}\left(\frac{T_{i+1 j k}-2 T_{i j k}+T_{i-1 j k}}{\Delta x^{2}}\right)+\left(\frac{T_{i j+1 k}-2 T_{i j k}+T_{i j-1 k}}{\Delta x^{2}}\right)+ \\ \left(\frac{T_{i j k+1}-2 T_{i j k}+T_{i j k-1}}{\Delta x^{2}}\right)\end{array}\right]$

Since the explicit scheme is used, the time step of thermal diffusion is limited by Fourier stability criteria, $\Delta t \leq \frac{\Delta x^{2}}{8 \alpha}$, where $\Delta x$ and $\alpha$ are the cell size and the thermal diffusivity respectively. The time step for solute diffusion is determined by the same criteria, but although by using the solute diffusivity $D$ rather than the heat diffusivity $\alpha$. Solving the thermal diffusion and solute diffusion in each time step is a multiphysical task, and the 
same time step should be used for both. Since the thermal diffusivity is much higher than the solute diffusivity, the thermal transport time step can be 100 times longer than the solute transport time step. The time step of the solute transportation is used to accelerate the computation, though the thermal diffusivity is slowed by dividing the thermal conductivity by 100 .

The algorithm to solve the heat transport equation requires: (1) initializing the spatial domain (i.e. the number of cells and the cell size), (2) initializing the domain with an initial temperature $\left(T^{0}\right)$; (3) specifying boundary conditions; (4) specifying alloy parameters such as heat capacity $C p$, latent heat $L h$, and thermal conductivity $K_{t h}$; (5) calculating the time step; and (6) starting time stepping. For each time step $i$ solve Equation-3.20 for $d H^{i}$ and

find $T^{i}=\frac{d H^{i}}{C p}+T^{i-1}$ for non-interfacial cells. The calculation is more complicated for interfacial cells.

\subsubsection{Curvature Computation}

Two different curvature calculation methods were implemented and compared in this study. The first uses a level set approach and the second uses cell counting. The accuracy of both methods was compared using a 'sphere test'.

\subsubsection{Curvature Computation by Cell Count Algorithm}

With the cell count method, the curvature of a cell is computed based on its solid fractions and those of neighboring cells. The definition of neighbor cells in three dimensions 
typically has 26 cells, as shown in Figure-3.8. The most popular used model was proposed by Nastac [52] as follows:

$$
\left.K_{r}=\frac{1}{a}\left(1-2 \frac{f_{s}+\sum_{i}^{26} f_{s_{S}}^{i}}{N+1}\right) \quad \text { [cell count curvature }\right]
$$

where, $N$ is the number of neighboring cells, $a$ is the cell size, $f_{s}$ is the solid fraction of the cell with the curvature evaluated, and $f_{s}^{i}$ is the solid fractions of neighbor cells.

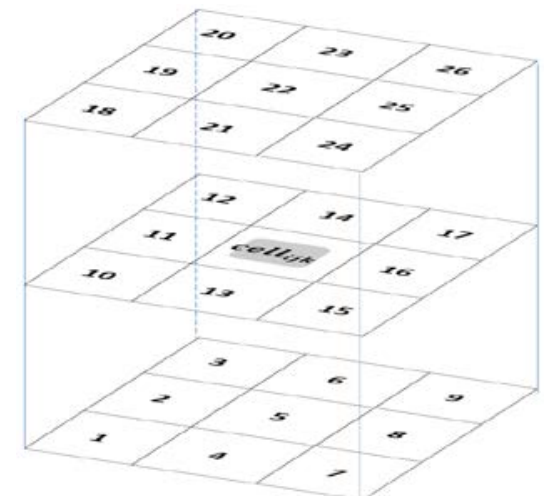

Figure-3.8: The neighbor cells definition for cell count method. The curvature is evaluated for the middle cell $\left(\right.$ cell $\left._{i j k}\right)$.

\subsubsection{Curvature Computation by the Level Set Method}

With this method, the curvature of a cell is estimated based on the gradient of solid fractions according to Equation-3.4. Marcias and Artemev [2] suggested that the solid fractions should be weighted by weighting factors $\left(w_{1}=0.2, w_{2}=0.1, w_{3}=0.05\right)$ to allow closer neighbor cells to have more effect on the curvature value. Using a weighted level set is helpful because it mitigates the sensitivity of curvature to the mesh by averaging the value 
of the calculated curvature. The definition of neighbor weights for a cell is shown in Figure3.9 .

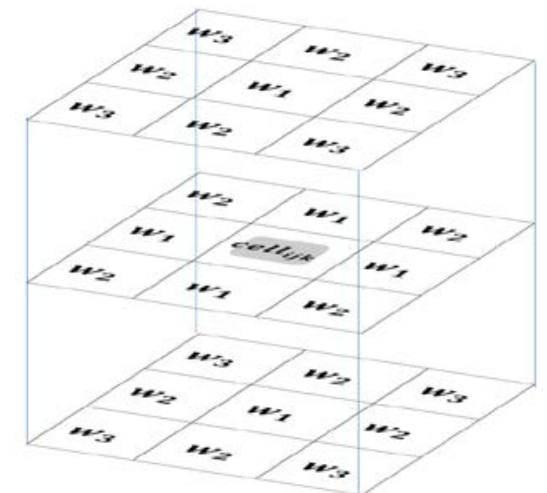

Figure-3.9: The neighbor weights for a centered cell Cell $_{i j k}$.

Each cell in the neighbor set should be weighted according to the following expression:

$$
\text { weighted } f^{s}=\frac{\sum_{i=1}^{27} w_{i} \cdot f_{s}^{i}}{\sum_{i=1}^{27} w_{i}} \quad \text { [weighted average solid fraction] }
$$

The algorithm for calculating curvature with the weighted level set method is:

- Specify the cell at which curvature should be evaluated, cell $_{i j k}$.

- Define its neighbor cells set (26 cells).

- Calculate the solid fractions $\left(f_{s}\right)$ of all cells, i.e., cell $_{i j k}$ and its neighbor cells.

- Weight each solid fraction by multiplying it by its weight factor, as in Equation3.22 .

- Calculate the curvature of cell $_{i j k}$ by solving Equation-3.4. 
The accuracy of curvature calculation of each method is evaluated using a computational test (called the sphere test) in which the estimated sphere curvature is compared with the analytical sphere curvature. Since with the level set method the weights of neighbor cells affect the calculation of the curvature, a searching optimization procedure is applied to optimize their values. The objective function is to minimize the difference between the estimated average curvature of all interface cells and the analytical curvature of a sphere. The optimization algorithm follows:

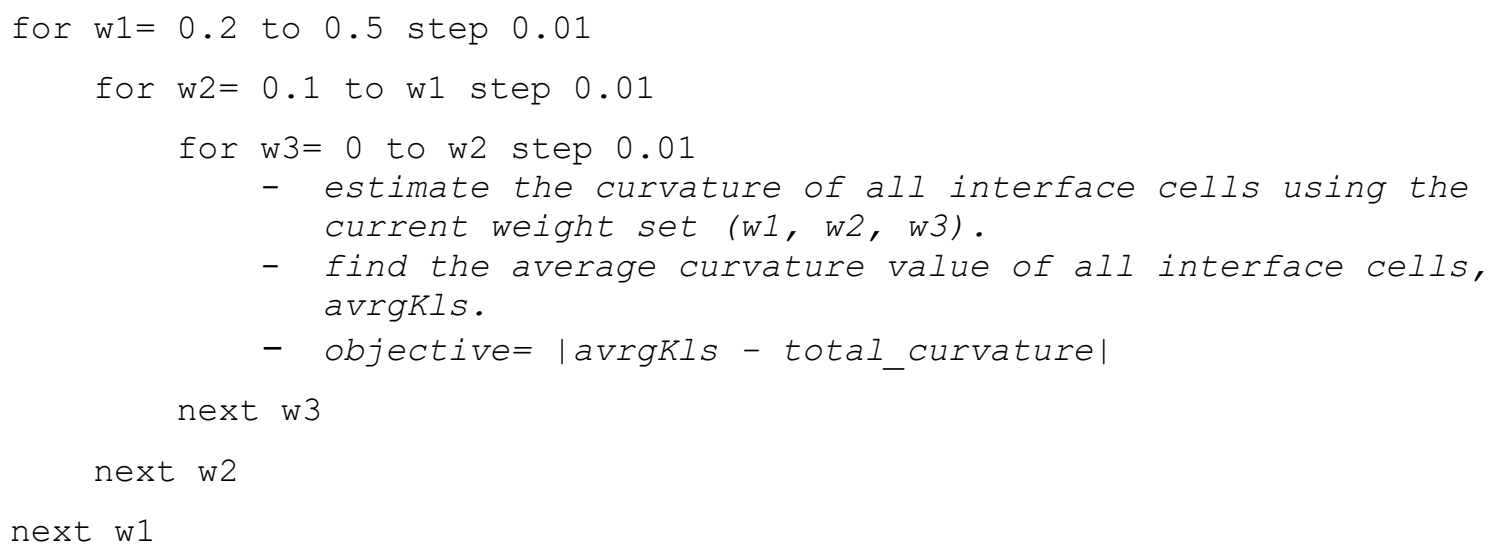

The algorithm finds the best weight set $\left(w_{1}, w_{2}, w_{3}\right)$ that minims the objective function. After running the algorithm for a sphere with a radius equal to four cells, the best weight values were $w_{1}=0.5, w_{2}=0.1$, and $w_{3}=0$.

\subsubsection{The Sphere Test}

In this test a sphere with a known radius is constructed in a cubic mesh and represents the $\mathrm{S} / \mathrm{L}$ interface. Any cell inside the sphere is considered completely solid, and any outside the sphere completely liquid. Cells at the sphere perimeter are interface cells, and thus their 
fraction of solid need to be estimated. In a dendrite simulation, the fraction of solids of interface cells are calculated based on thermodynamics. However, for the sphere test, the fraction of solids for all interface cells are estimated by generating random points inside each cell. For example, if 100 points are randomly and uniformly generated inside an interface cell, its solid fraction is equal to the number of points inside the sphere over the total number of points, as shown in Figure-3.10. The curvatures of interface cells are, then, estimated based on this solid fraction using the level set and cell count methods. The accuracy of the curvature estimation is determined by comparing it against the analytical curvature of the sphere.
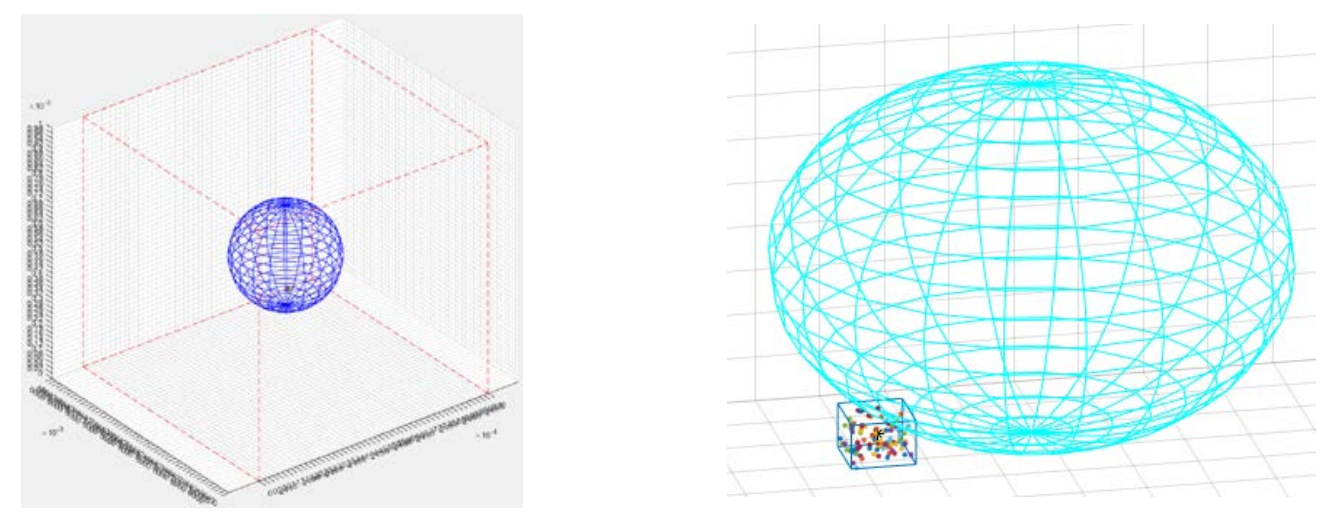

Figure-3.10: The estimation of solid fraction for the sphere test.

A series of sphere tests are conducted to evaluate the accuracy of the curvature estimation of the cell count and level-set methods. Ideally, the estimated curvature for any interfacial cell should equal the analytical curvature. However, both methods cannot calculate this result, so each cell will have different curvature value. Thus, the average estimated 
curvature $\overline{K r}$ of all interface cells is the used measurement, and the accuracy of the method increases as the $\overline{K r}$ value approaches the analytical curvature.

Sphere tests with 20 different sphere radii are investigated to evaluate curvature computations. The size of the computational domain is $50 \times 50 \times 50$ cells with cell size equal 1e- 6 , and the sphere radius varies from 1 cell to 20 cells. The analytical sphere curvature equal 2/sphere radius, and the value of $\overline{K r}=\sum_{i}^{s c} K r_{i} / n o . s c$, where $s c$ is the cell on the sphere surface. It was determined that using 100 random points to estimate the solid fraction is adequate, and this reduces the computational time. Figure-3.11 shows the error of curvature estimation using cell count, level-set with no averaging, level-set with a weight averaging-, and level-set with the optimum weight averaging.

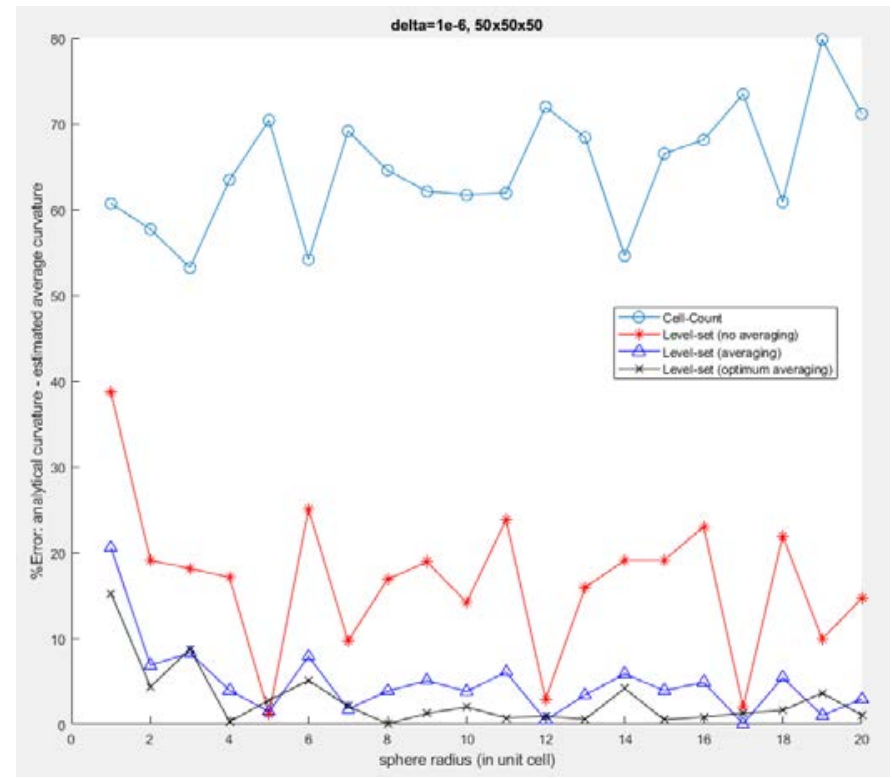

Figure-3.11: The error of curvature estimation by different curvature calculation methods. 
Regarding the Figure-3.11, the error of curvature estimation using the cell count method is more than $60 \%$ according to the sphere tests. However, this method is widely used because of its simplicity and lower computational requirements. The figure shows that level-set method is more accurate, and the curvature estimation error is approximately $20 \%$. In addition, if the averaging of solid fractions is used, the estimation error reduces to $10 \%$. Moreover, the averaging procedure with optimum weights produces more accurate estimation, as illustrated by the difference between the lowest two curves. Investigation of the level set method indicates that it is more accurate than the cell count method. This is because the level set method is based on the geometrical differentiation, and it considers the neighbor cell effects. However, the level set method with averaging is more computationally expensive than the cell count method.

\subsubsection{Solid Fraction Computation Tool}

The solid fraction of any interfacial cell during a simulation is obtained from a stored CALPHAD data structure, as will be explained in section-3.5. During simulation each interfacial cell has a specific thermodynamic parameter (i.e. temperature and solute concentrations). According to a cell's thermodynamic parameters, the solid fraction of the cell is read from the stored CALPHAD data and assigned to the cell. In the case of imposed temperature values, the solid fraction can be obtained directly from the CALPHAD data. Alternatively, if the enthalpy method is used to calculate the temperature field, the solid fraction should be obtained by an iteration scheme that specifies how much the enthalpy 
changes the sensible heat, and how much it changes the phase fractions. This iteration scheme is called in this thesis one cell solidification module (OCSM). The output of the heat diffusion solver is the change in enthalpy, $d H$. If the amount of $d H$ reduces a cell temperature below the liquidus temperature, a portion of $d H$ will increase the solid fraction. The objective of OCSM is to find out the cell temperature and solid fraction according to the given $d H$. Figure-3.12a schematically shows the iteration inside the OCSM for a binary alloy, and the same procedure is used for higher order alloys.

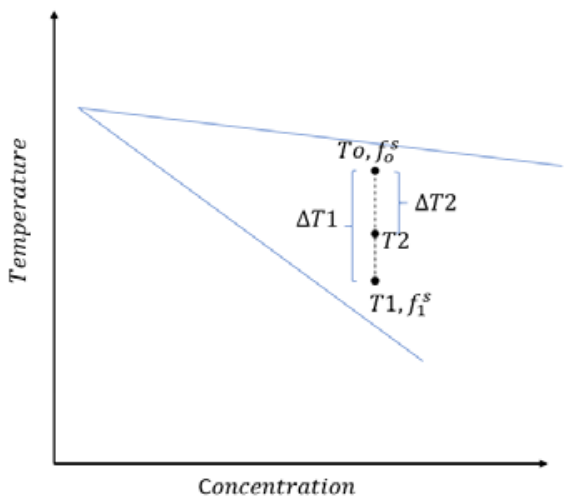

(a)

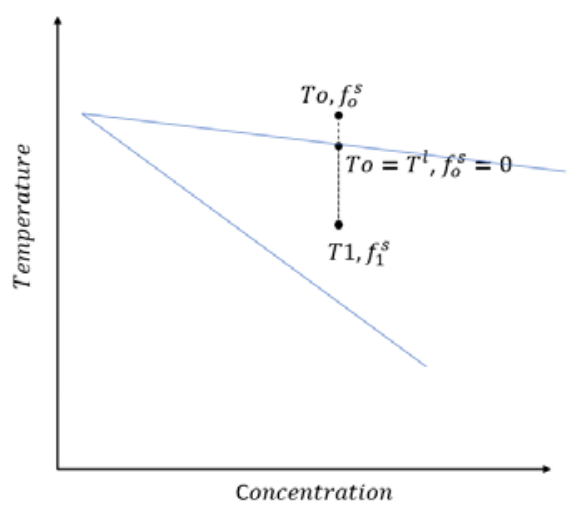

(b)

Figure-3.12: Pseudo binary liquidus and solidus lines to illustrate OCSM.

If $T_{o}$ and $f_{o}^{S}$ are the initial temperature and solid fraction of an interfacial cell, it is assumed that the given $d H$ reduces the temperature by $\Delta T 1$. Furthermore, the solid fraction at the first iteration ( $i=1) f_{i}^{s}$ according to $T 1_{i}$ is read from CALPHAD data. Then, the total enthalpy is calculated according to:

$$
d H_{\text {total }}=\left(T 1_{i}-T_{o}\right) \cdot C_{P}+\left(f_{o}^{s}-f_{i}^{s}\right) \cdot L h \quad e q 3.23
$$


Since solidification occurs due to $\Delta T 1$, some heat will be released in the cell and increase the temperature from $T 1$ to $T 2$; thus, $\Delta T 2=\Delta T 1 \cdot \frac{d H}{d H_{\text {total }}}$. When $\Delta T 1 \approx \Delta T 2$, the iterations end, and the cell temperature is equal to $T 2$ and its solid fraction is read from CALPHAD data $\left(f_{i}^{S}\right)$. If the condition is not satisfied, the iteration continues $(i=2, \cdots)$ until $\Delta T 1 \approx \Delta T 2$. At the onset of a cell solidification, the cell temperature is in the liquid state $\left(T_{o}\right)$ and its initial solid fraction $\left(f_{o}^{S}\right)$ is equal to zero (Figure-3.12b). In this case, the OSCM set the initial temperature to be the liquidus temperature and the same OSCM procedure continues.

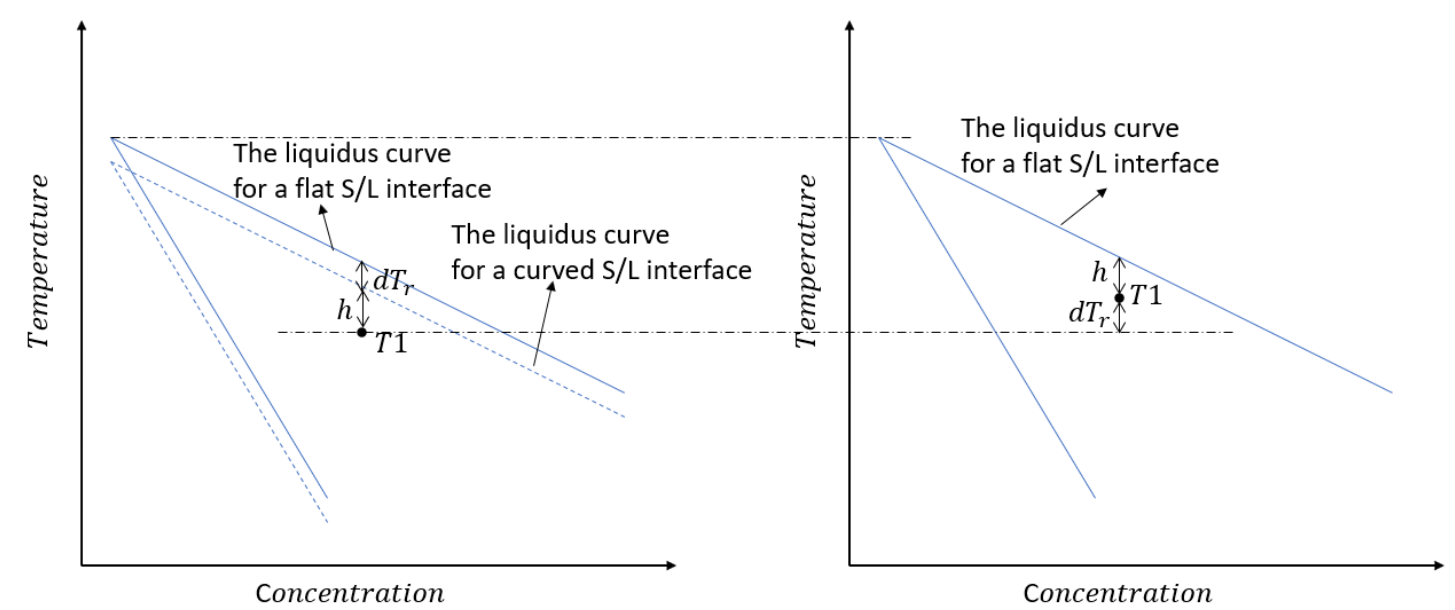

(a)

(b)

Figure-3.13: Incorporation the effect of capillarity undercooling for reading from CALPHAD data.

The stored thermodynamic data are for a flat $\mathrm{S} / \mathrm{L}$ interface, so the effect of the capillarity undercooling $d T_{r}$ should be incorporated during reading the information from thermodynamic data. Incorporating the capillarity undercooling can be obtained by either shifts down the liquidus curve by $d T_{r}$ (Figure-3.13a) or shifts up the temperature $T 1$ by 
$d T_{r}$ (Figure-3.13b). The second option is more applicable to be used. For example, if a temperature of an interfacial cell is calculated by the heat diffusion solver $T 1$ and $d T_{r}$ is calculated by the curvature calculation, the used temperature value of reading from CALPHAD data is equal to $T 1+d T_{r}$.

\subsection{Coupling CALPHAD Data to Cellular Automata Modeling}

The solidification model requires relationships between thermodynamic parameters and phase fractions, which are obtained from the precalculated and stored CALPHAD calculation. Thermodynamic information calculated at the intended temperature range by the computational code includes: the equilibrium concentration of chromium $C_{c r}^{l}$ in liquid; the equilibrium concentration of carbon $C_{c}^{l}$ in liquid; the equilibrium concentration of chromium $C_{c r}^{S}$ in solid; and the equilibrium concentration of carbon $C_{c}^{S}$ in solid. At a constant temperature, the combination of $C_{c r}^{l}$ and $C_{c}^{l}$ describes the liquidus boundary, and the combination of $C_{c r}^{S}$ and $C_{c}^{S}$ describes the solidus boundary.

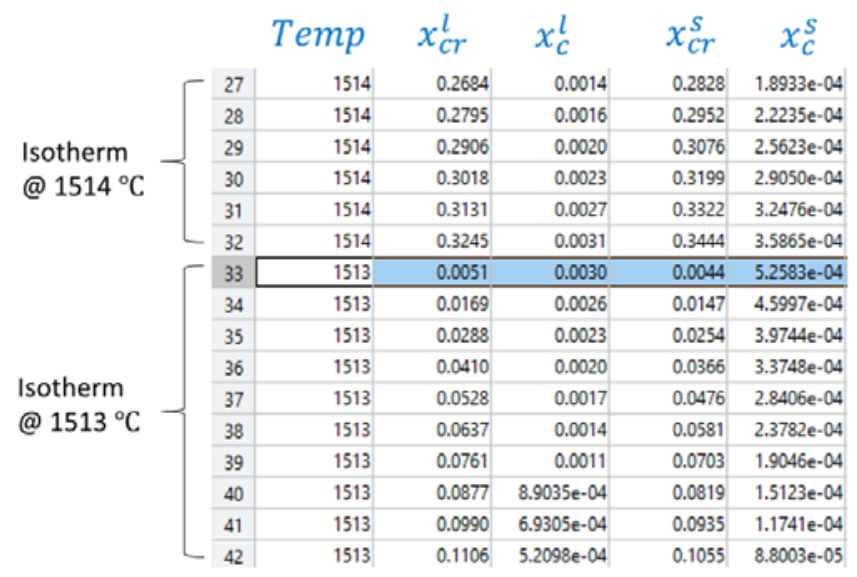

Figure-3.14: Thermodynamics lookup table. 
The thermodynamic data for the temperature range 1494 to $1537^{\circ} \mathrm{C}$ is stored in a database, as shown in Figure-3.14. Another table with the same structure is calculated for the temperature range 1330 to $1494{ }^{\circ} \mathrm{C}$. Each row in the table defines a tie line at the corresponding temperature, and all rows with the same temperature define an isotherm.

Thermodynamic data at any temperature that is not stored in the database can be interpolated from the stored data. For example, if the table stores data at temperatures of 1511 and $1510.5^{\circ} \mathrm{C}$, the data of the isotherm at $\mathrm{T}=1510.7^{\circ} \mathrm{C}$ can be linearly interpolated from the stored data.

The CA solidification model must define the thermodynamic information for each cell at every time step of the simulation. The most direct way to achieve this is to apply CALPHAD calculation to each cell for the entire simulation. However, performing these calculations during a simulation is problematic as the computationally cost is prohibitively high. The solution is to pre-calculate and store enough thermodynamic information for the intended accuracy, thereby allowing the solidification model to search the stored data and do interpolations during the simulation, as shown in Figure-3.15.

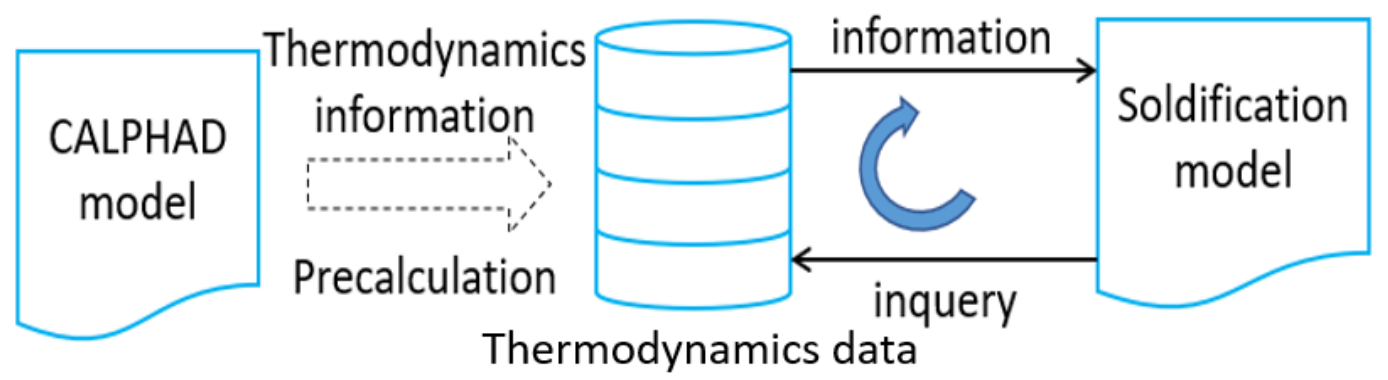

Figure-3.15: The interaction between the solidification and CALPHAD models. 
The coupling algorithm starts with defining the alloy properties and simulation parameters, then the computational domain (CD) is initialized. The simulation proceeds by calculating the heat transport, solute transport, capillarity undercooling-, and solid fraction at every time step. A detailed explanation of these steps follows.

\subsection{Obtaining Information from the CALPHAD Data}

Any thermodynamic state (i.e. stable phases, the fraction of phases, the equilibrium composition of elements) is defined by its temperature and average composition. The solidification model requires a relationship between thermodynamic states and solid fractions, and the solid fraction can be interpolated from the stored CALPHAD data structure. Two techniques of storing, searching, and interpolating thermodynamic information are proposed and investigated in this study. The first technique is based on scanning a lookup-table and interpolating tie lines information. The lookuptable stores a collection of non-structured tie lines. The second technique is a pointwise interpolation scheme which stores a structured thermodynamics data. In addition, the possibility of representing phase diagrams using Bezier's curves is also investigated.

\subsubsection{Scanning Procedure for Reading Solid Fractions}

The scanning procedure is explained next using the lookup table of Fe-Cr-C thermodynamics data. Any thermodynamic state can be described by three coordinates: temperature, chromium composition, and carbon composition $(C c r, C, T)$. Now, consider three different query points (qP1, qP2, and qP3) that represent three thermodynamic states, 
as shown in Figure-3.16. The $\mathrm{X}$ and $\mathrm{Y}$ coordinates of each point represent its chromium and carbon composition, and the $\mathrm{Z}$ coordinate represents the state temperature. Since the stored CALPHAD data explicitly defines the phase boundary liquidus and solidus curves, the procedure can easily locate any state by comparing its coordinates to the boundary curve points.

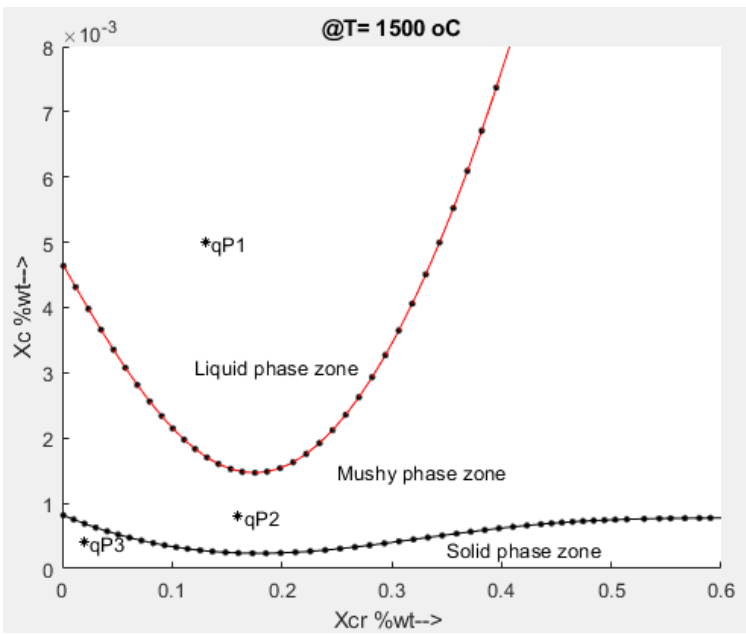

Figure-3.16: Three different thermodynamic states: liquid (qP1), solid (qP3), and mushy (qP2).

If a given query point $\mathrm{qP}$ is in the liquid phase (e.g. qP1), its solid fraction equals zero and its liquid fraction equals one. As well, the concentration of chromium and carbon in the liquid is equal to the initial alloy composition. Reversely, if $\mathrm{qP}$ is in the solid phase (e.g. $\mathrm{qP} 3$ ), its solid fraction equals to one, and its liquid fraction equals to zero. In addition, the concentration of chromium and carbon in the solid phase is equal to the initial alloy composition. In the third case (qP2), the solid and liquid fractions should be interpolated by following the procedure outlined in Figure-3.17. 
1) Locate the four nearest points to the given $q \mathrm{P}$ using the inQuad locating procedure.

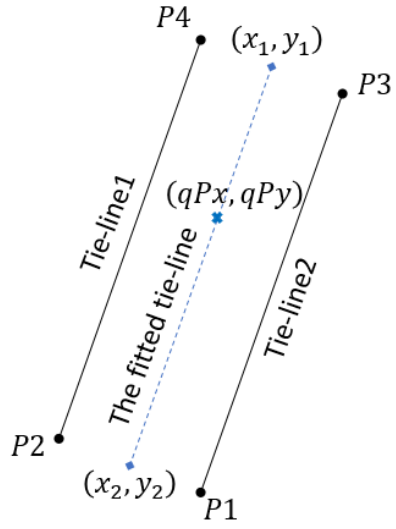

2) Construct two tie-lines, Tie-line1 and Tie-line2, using the nearest points.

3) Fit a parallel tie-line to the constructed tie-lines.

4) The points $\left(\mathrm{x}_{1}, \mathrm{y}_{1}\right)$ and $\left(\mathrm{x}_{2}, \mathrm{y}_{2}\right)$ can then be calculated using linear geometrical relations, where, $\mathrm{x}_{1}, \mathrm{y}_{1}, \mathrm{x}_{2}$ and $\mathrm{y}_{2}$ are the equilibrium compositions of chromium and carbon in liquid and solid.

5) The solid fraction is equal to $s F=(x 1-q P x) /(x 1-x 2)$ and the liquid fraction is equal to $\mathrm{lF}=1-\mathrm{sF}$.

Figure-3.17: The procedure of calculating the information of a query point ( $\mathrm{qP}$ ) in the mushy phase.

The inQuad locating procedure defines a convex quadrilateral shape which eases the locating procedure. The area of any given quadrilateral shape is the sum of two right triangles, as shown in Figure-3.18a. In addition, a given qP can construct four triangles with a quadrilateral shape vertex. Thus, qP can be located as shown in Figure-3.18. The area of a triangle with known vertex coordinates can be calculated using Heron's formula.
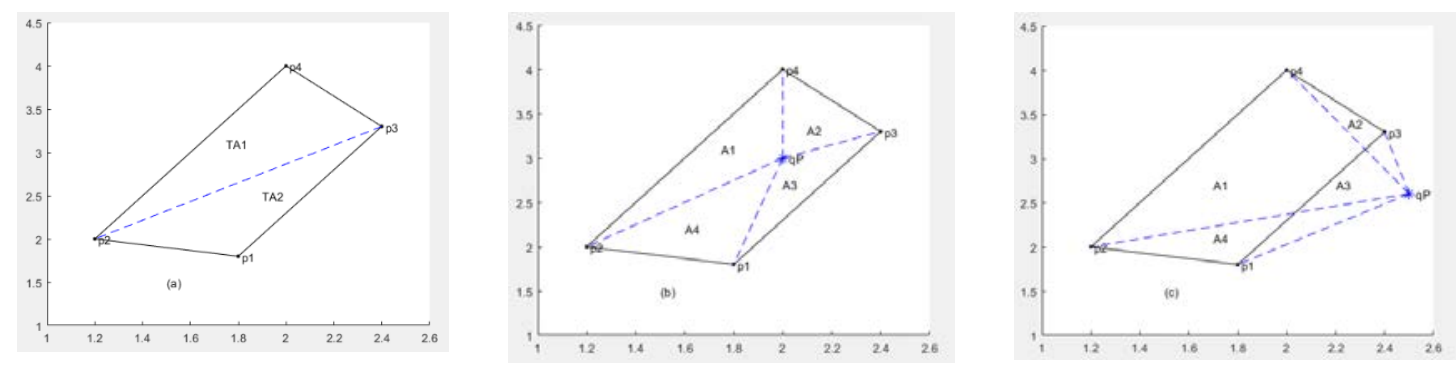

Figure-3.18: The inQuad locating procedure. (a) the quadrilateral shape area $=\mathrm{TA} 1+\mathrm{TA} 2$, (b) $\mathrm{qP}$ is inside the shape if $\mathrm{A} 1+\mathrm{A} 2+\mathrm{A} 3+\mathrm{A} 4<=$ quad area, (c) $\mathrm{qP}$ is outside the shape if $\mathrm{A} 1+\mathrm{A} 2+\mathrm{A} 3+\mathrm{A} 4>$ quad area. 
The query points in Figure-3.16 are at specific temperatures or isotherms, since the thermodynamic database stores many isotherms at different temperatures. If a given point qP has a temperature value (qT) that is not stored in the database, the information at this temperature can be linearly interpolated between two stored isotherms: the first one is at temperature Ttop and the second one is at temperature Tbot. For example, if the database stores information at Ttop $=1511{ }^{\circ} \mathrm{C}$ and $\mathrm{Tbot}=1510.5{ }^{\circ} \mathrm{C}$, and the given point is $\mathrm{qT}=1510.7^{\circ} \mathrm{C}$, the information at the query temperature can be interpolated as shown in Figure-3.19.

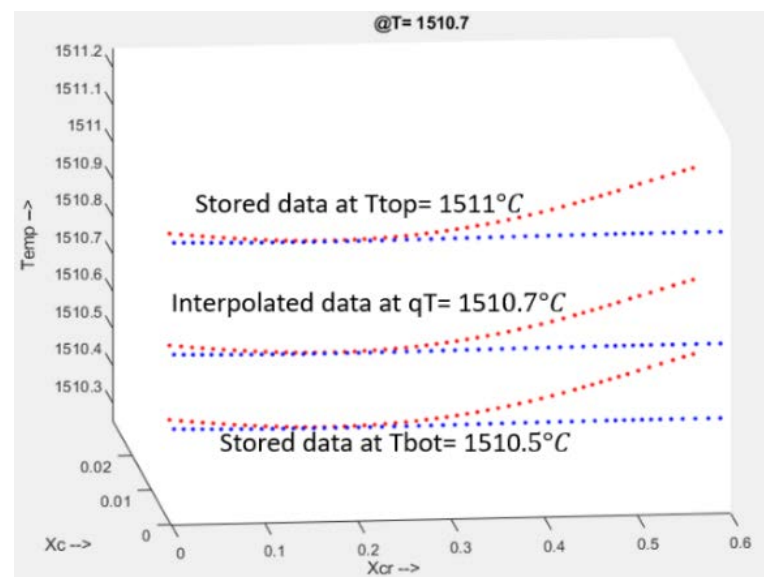

Figure-3.19: Interpolate data between two different temperature values.

While the calculation of a single point equilibrium takes approximately $0.1 \mathrm{sec}$ to calculate the thermodynamic information, the lookup-table procedure takes $0.0003 \mathrm{sec}$ to interpolate the information. 


\subsubsection{Pointwise Interpolation Procedure for Reading Solid Fraction}

With this technique, the thermodynamic data are stored in a structural mesh, and the distance between all data points is equal in every direction, as shown in Figure-3.20. Each point has specific information, including solid fraction, liquid fraction, and the equilibrium composition of each element in every phase. The advantage of using the structural mesh is that the location of a given inquiry point qP can be found swiftly by knowing its coordinate; thus, the eight neighbor data points of $\mathrm{qP}$ can be determined directly.

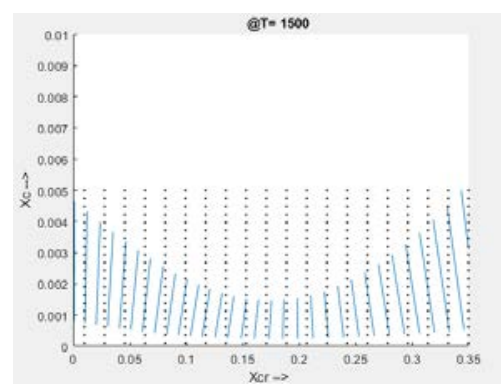

Figure-3.20: Data points that store thermodynamic information at $\mathrm{T}=1500^{\circ} \mathrm{C}$.

The interpolation value of $\mathrm{qP}$ (the solid fraction) can be interpolated from the eightneighbor data points by using the inverse weighted distance scheme shown in Figure-3.21.

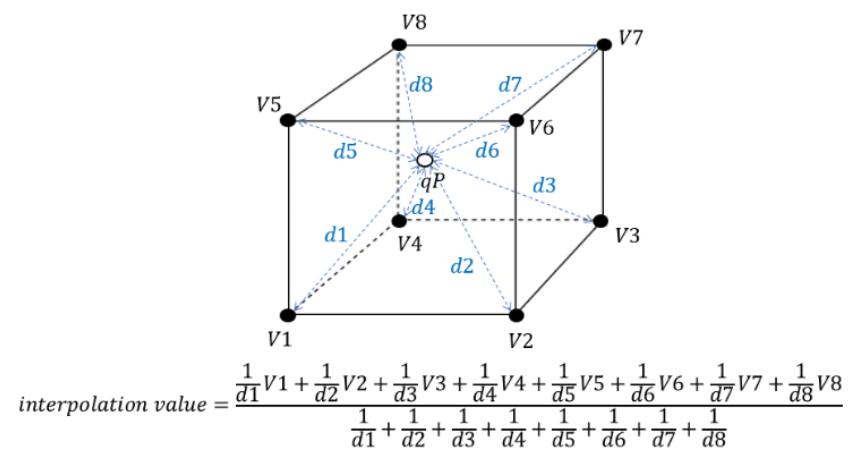

Figure-3.21: The inverse weighted distance scheme. 
This interpolation scheme can be used to interpolate all thermodynamic information. Although this interpolation scheme is faster than the former one, it is found that the interpolated information slightly violates the lever rule and more data storage should be used to improve the accuracy. For example, it is founded that even with data storage of 50 $\mathrm{MB}$, the interpolated information slightly violates the lever rule. The violation of lever rule causes mass leakage during the solidification simulation.

\subsubsection{Bezier Curves Representation of Phase Diagrams}

A Bezier curve is a parametric curve that uses Bernstein polynomials as a basis. Recently, some researches proposed the possibility of storing a phase diagram in a set of Bezier curves. For example, the paper [63] showed that the Bezier curve is a simple, efficient, and accurate way to represent and store the phase diagram of Al-Zn binary system.
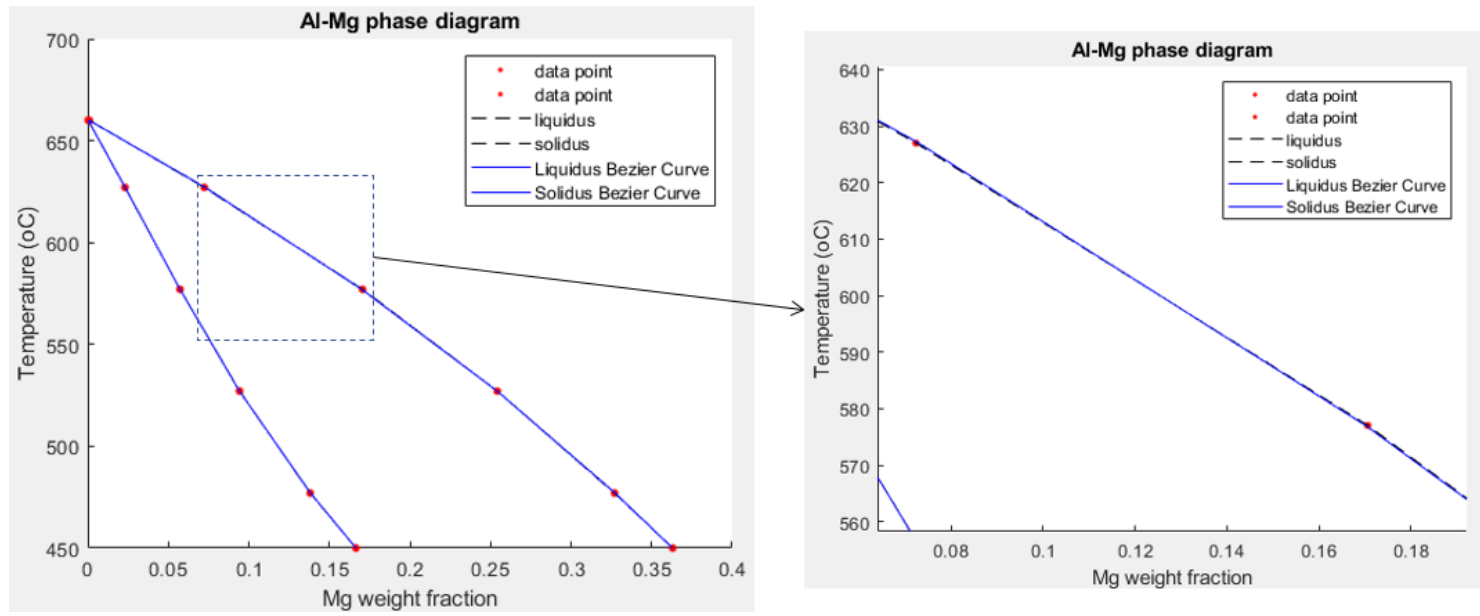

Figure-3.22: Representing the solidus and liquidus of Al-Mg by Bezier curve.

In this study, the possibility of reading thermodynamic information from the Bezier curves of an Al-Mg binary diagram is investigated. The mathematical formulation is described in 
[33], and the information of the solidus and liquidus boundaries are obtained using MatCalc ${ }^{\circledR}$. A MATLAB code is developed to construct the Bezier curves description of Al-Mg solidus and liquidus as shown in Figure-3.22. Figure-3.23 shows three different possibilities of reading solid fractions for a given thermodynamic state.

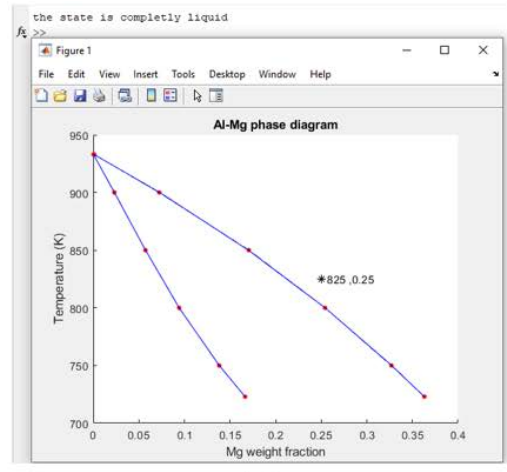

(a)

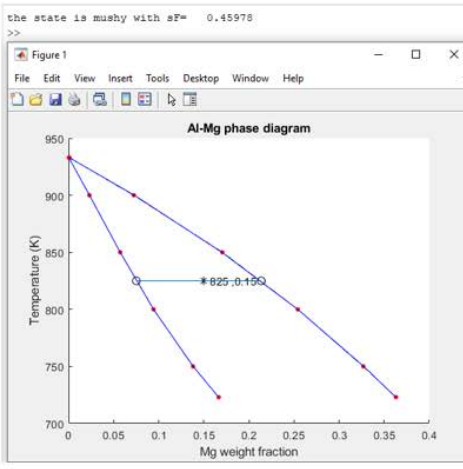

(b)

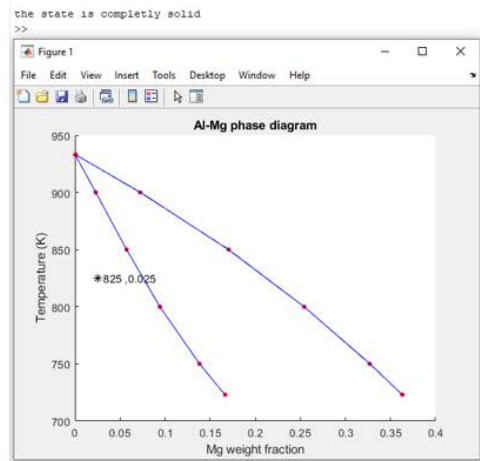

(c)

Figure-3.23: Reading the solid fractions from a Bezier curve. (a) liquid state, (b) mushy state, and (c) solid state.

\subsection{Alloy Properties and Simulation Parameters}

The investigated alloy in this study is $\mathrm{Fe}-\mathrm{Cr}-\mathrm{C}$ stainless steel. The required alloy properties are chromium diffusion coefficient $D_{c r}$, carbon diffusion coefficient $D_{c}$, heat capacity $C_{P}$, thermal conductivity $K_{t h}$, latent heat of fusion $L h$, Gibbs-Thomson coefficient $\Gamma$, and anisotropy strength $\in$. In addition, the simulation parameters are the number of cells in $\mathrm{x}$, $\mathrm{y}$, and $\mathrm{z}$ directions (i.e. the size of a computational domain), the cell size $\Delta x$, the nominal alloy composition $\left(C_{c r}^{o}, C_{c}^{o}\right)$, the initial undercooled temperature $T_{0}$, the rate of cooling $R C$, the temperature gradient within the computational domain $T G$. 


\subsection{Computational Domain (CD) Initialization}

The initialization of $\mathrm{CD}$ means assigning the initial field variables (i.e. temperature, composition, and solid fraction) to all the CD cells and boundary conditions. An initial state is hypothetical, as it doesn't belong to a specific physical process; thus, some fluctuation is expected at the beginning of the simulation. With time, the system achieves stability and proceeds with steady state. Figure-3.24 diagrams CD initialization.

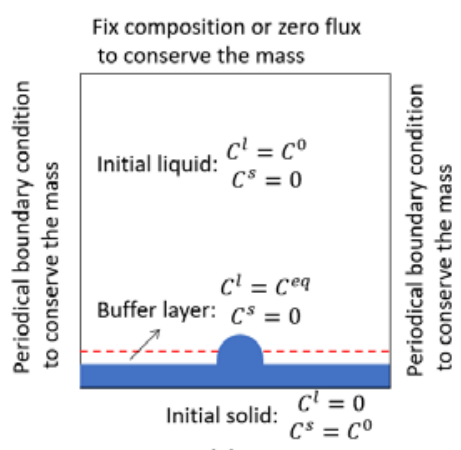

(a)

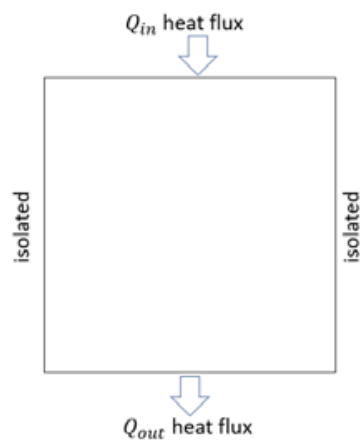

(b)

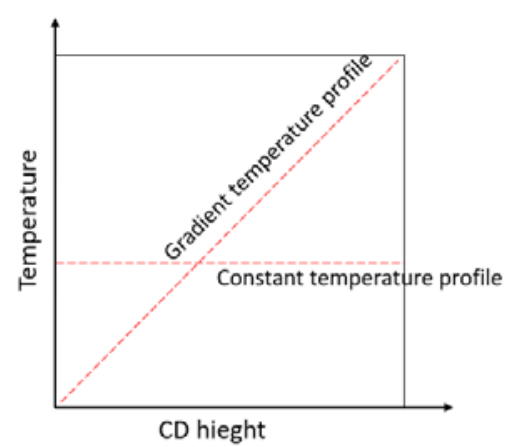

(c)

Figure-3.24: The initialization of a computational domain.

The upper and the lower boundaries of the $\mathrm{CD}$ are closed, and all side boundaries are periodical. These two conditions conserve the mass within the $\mathrm{CD}$ (the thermal boundary conditions are shown in Figure-3.24b). Two cases can be considered for thermal boundary conditions. In the first case a temperature profile with a zero or constant cooling rate is imposed on all cells [62]. In this case, there is no need to compute the heat diffusion equation since the heat diffusion is much faster than solute diffusion and the temperature quickly becomes uniform. However, for more accurate results, the second thermal boundary condition is also applied, as shown in Figure-3.24c. This shows the boundary 
condition with a heat flux entering the CD from the top and another heat flux leaving the CD from the bottom.

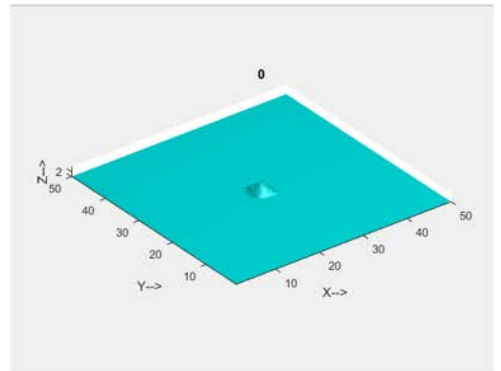

(a)

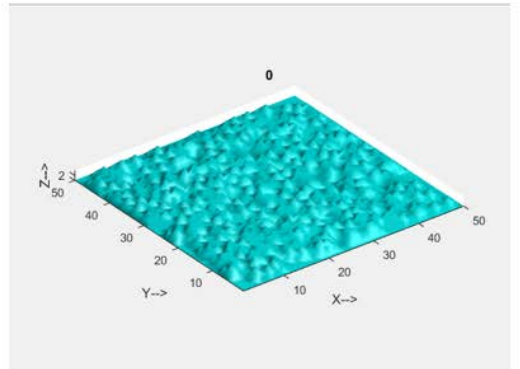

(b)

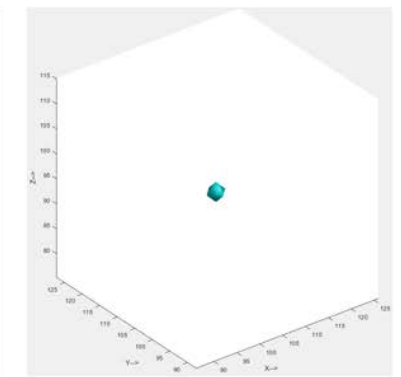

(c)

Figure-3.25: Three different initial solid/liquid interface for simulations.

The CD can be initialized by three different $\mathrm{S} / \mathrm{L}$ interfaces, as shown in Figure-3.25. The first studies the dendritic or cellular growth, and the second studies the equiaxed growth. In these configurations, most $\mathrm{CD}$ cells are liquid with zero fraction of solid, and the others are completely solid to represent the initial topology of the S/L interface. The solid composition of all solid cells equals the initial alloy composition, $C^{s}=C^{o}$. In addition, the liquid composition of all liquid cells equals the initial alloy composition, $C^{l}=C^{o}$. The initial composition of the liquid can be undercooled for a specific degree. Figure-3.26a shows an initial undercooled liquid state, and Figure-3.26b shows an initial nonundercooled liquid state. It is important not to use a highly undercooled liquid state, as this will produce an aggressive growth rate not suitable for welding processes (low Peclet regime). Finally, a buffer zone of a specific height can be added to cover the initial solid cells. The composition of the buffer zone equals to the equilibrium composition of the 
initial temperature $T o$ and the composition $C_{c r}^{o}, C_{c}^{o}$. The buffer zone will suppress any noise growth from the flat $\mathrm{S} / \mathrm{L}$ interface.

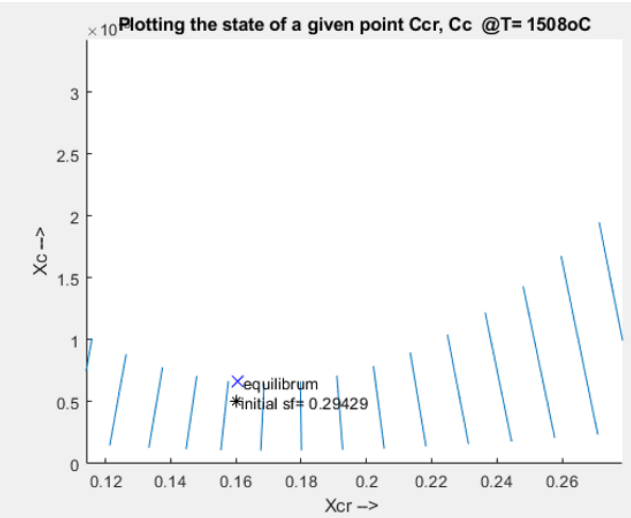

(a)

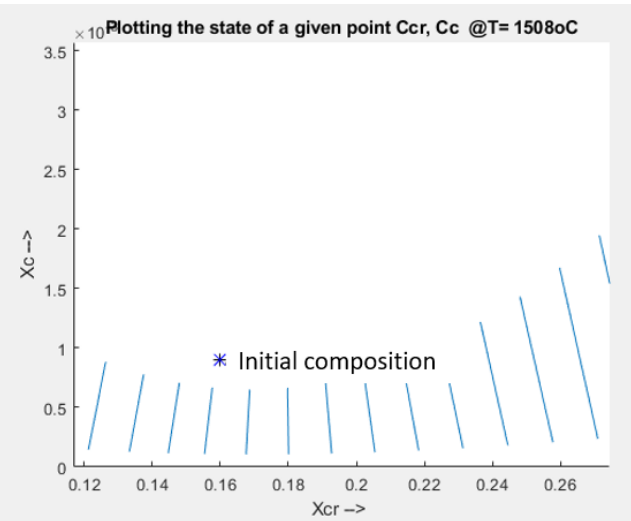

(b)

Figure-3.26: (a) undercooled and (b) non-undercooled initial alloy composition.

\subsection{The Algorithm of the Simulation}

The entire simulation code is written as a modular structure, and each computational tool is written in a separate routine. For example, the computation of a thermal field uses a specific routine, while the interpolation of thermodynamics information occurs in another routine, and so on. Every simulation is terminated whenever the ending condition is satisfied; that is, if a tip of the growing dendrite reaches $90 \%$ of the computational domain height. The main body of the code calls a routine when it is required, as shown next. 


\section{The algorithm of the simulation}

1: Define the alloy properties.

2: Define the simulation parameters.

3: Initialize the computational domain variables: $f^{s}, T o, C_{c r}^{l}, C_{c}^{l}, C_{c r}^{s}, C_{c}^{s}$, curvature.

4: Calculate the time step $\Delta t$ and start the time stepping:

Call [Temp routine] to apply an imposed temperature profile to get newT.

5: $\quad$ OR

Call [Heat Transport routine] to compute the heat diffusion by the enthalpy method to get $d H$.

6: Compute the mass diffusion to get the new composition distribution: $\left[\operatorname{new}_{c r}^{l}, \operatorname{new} C_{c}^{l}\right]$. Call [phase evolution routine] to compute the solid fraction $f^{s}$ in the CD using newT, new $C_{c r}^{l}$, new $C_{c}^{l}$.

7: $\quad$ OR

Call [OCSM] if the enthalpy method is used to compute the solid fraction $f^{s}$ and temperature field newT.

8: Call [Curvature routine] to compute the new curvature of the $\mathrm{S} / \mathrm{L}$ interface.

9: $\quad$ Update all variable fields for all cells.

10: Check the simulation ending condition.

11: Next time step. 


\section{The algorithm of OCSM}

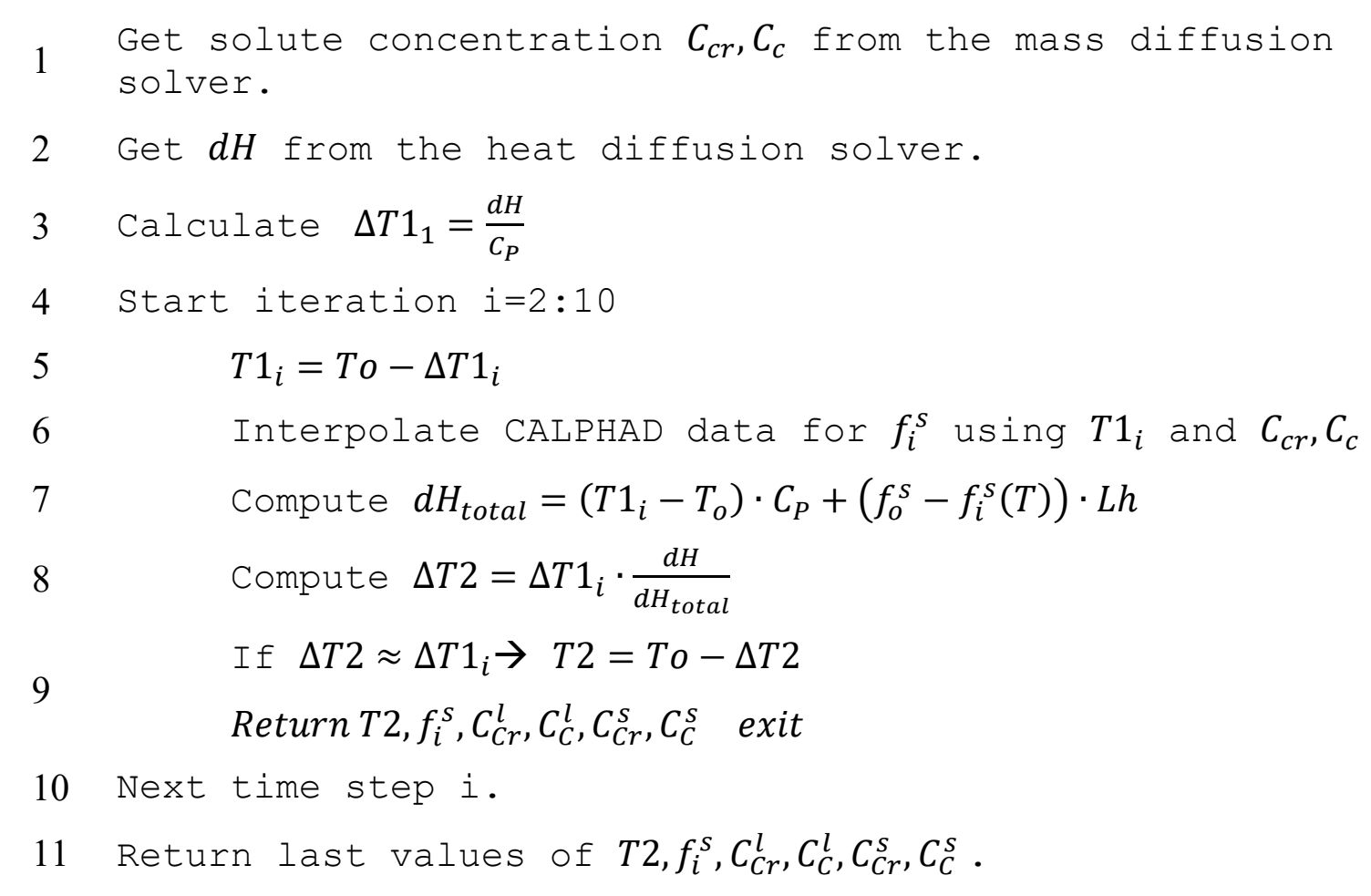

\subsection{Model Verification and Validation}

\subsubsection{Mass Conservation}

To validate the model, the mass conservation was investigated by assigning an initial value of chromium and carbon in weight fraction $\left(C_{c r}^{o}\right.$ and $\left.C_{c}^{o}\right)$ for every cell in the computational domain. If a cell is initially liquid, the initial values of $C_{c r}^{o}$ and $C_{c}^{o}$ are assigned to the cell liquid phase, and the initial values are assigned to solid phase cell if it is initially solid. Therefore, the total amount of chromium and carbon in the entire domain can be calculated. At the end of each time step during a simulation, the total mass of chromium and carbon are recalculated and compared to the initial total mass, and mass is conserved if the 
difference between the initial mass and the recalculated mass is close to zero. The total mass of solutes can be calculated as follows:

$$
\begin{aligned}
C_{c r}^{\text {total }} & =\sum_{i}^{\text {no.Cells }} C c r_{i}^{l} \cdot f_{i}^{l}+C c r_{i}^{s} \cdot f_{i}^{s} \\
C_{c}^{\text {total }} & =\sum_{i}^{\text {no.Cells }} C c_{i}^{l} \cdot f_{i}^{l}+C c_{i}^{s} \cdot f_{i}^{s}
\end{aligned}
$$

Mass conservation is perfect in this model, as the maximum difference between the initial solute mass and the solute mass during the simulation is approximately $10^{-9}$ of the weight fraction.

\subsubsection{Simulated Dendritic Structures}

Solidification theory and observations predict a parabolic dendrite tip shape [15]. Unlike the dendrite body, close to the tip, the $\mathrm{S} / \mathrm{L}$ interface is smooth under steady-state growth (Figure-1.9). This feature should be captured by the solidification model. Figure-3.27 shows a dendrite of $\mathrm{Fe}-\mathrm{Cr}-\mathrm{C}$ from the model developed in this study. In addition, Figure3.28 shows a comparison between the obtained simulation result and a published micrograph result. 


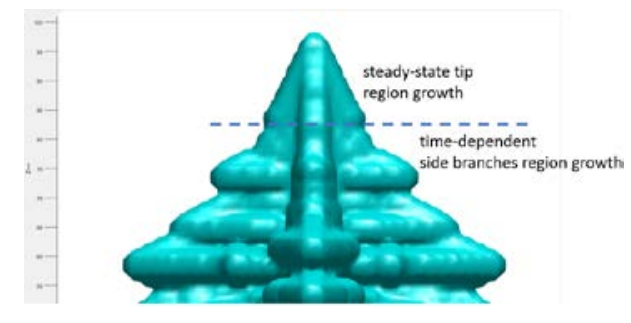

Figure-3.27: The resulting dendrite tip.
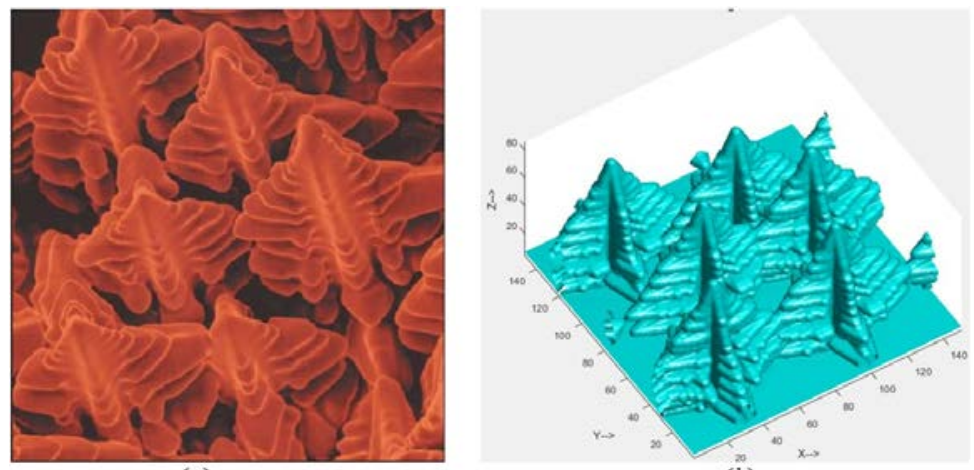

(a)

(b)

Figure-3.28: (a) a scanning-electron micrograph of dendrites in a weld [64], and (b) the solidified microstructure of Fe-C-Cr [the developed model].

The equiaxed growth of the developed model is compared with the results of [64]. Figure-

3.29 shows the results of the developed CALPHAD model and the results from [65].

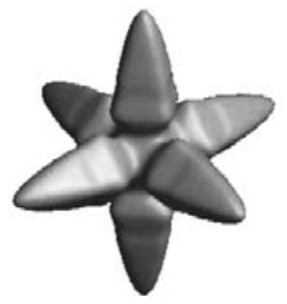

(a)

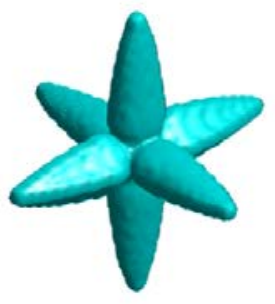

(b)

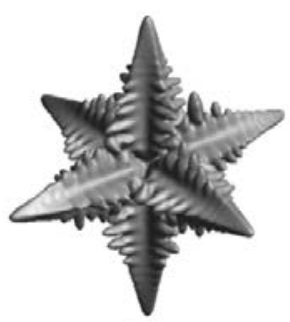

(c)

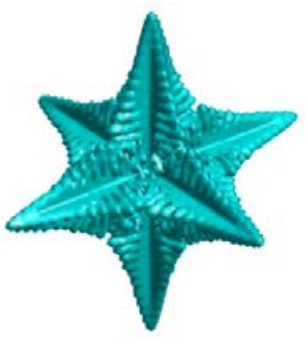

$(d)$

Figure-3.29: (a) and (b) show equiaxed dendrites with low anisotropy, with (a) from [65] and (b) from the developed CA-CALPHAD model. (c) and (d) show equiaxed dendrites with high anisotropy, with (c) from [65] and (d) from the developed CACALPHAD model. 


\subsubsection{The Selection of Cell Size}

In $\mathrm{CA}$ simulation, the growth of a dendrite including the formation of secondary and tertiary arms, is controlled by two anisotropy effects: mesh anisotropy and surface energy anisotropy. Ideally, the effect of the mesh anisotropy should be eliminated, but this is not possible since the mesh exists. The effect of the mesh anisotropy decreases as the cell size decreases and the growth is more controlled by the effect of surface energy anisotropy. However, decreasing the cell size increases the number of cells and decreases the time step since an explicit numerical scheme is used. Therefore, the cell size should be selected to be large enough to speed up the simulation and small enough to reduce the effect of mesh anisotropy and allow the surface energy anisotropy to influence the growth. In order to select a proper cell size, some simulation tests with the same simulation parameters and different cell sizes are investigated to relate the dendrite tip radius and the cell size. The used simulation parameters are nominal alloy composition $10^{-6} \mathrm{wt} \%$ of $\mathrm{Cr}$ and $0.009 \mathrm{wt} \%$ of $\mathrm{C}$, initial temperature $=1517^{\circ} \mathrm{C}$, anisotropy coefficient in Z-direction $=0.2$, anisotropy coefficient in XY-plane $=0$, and Gibbs-Thomson coefficient $\Gamma=1^{-7} K \cdot m$. The used cell size $(\mathrm{dx})$ for the simulation one to nine is $0.05,0.1,0.15,0.2,0.25,0.3,0.5,0.75$, and 1 $\mu \mathrm{m}$. Each simulation produces a dendrite with different tip radius TipR, even though the used simulation parameters are the same, because of the interaction between the mesh anisotropy and the surface energy anisotropy. The tip radius of each dendrite is calculated from the curvature value $(\mathrm{Kr})$ of the topmost cell at the end of the simulation as $\operatorname{TipR}=2 / \mathrm{Kr}$.

The calculated TipR are plotted with the corresponding $d x$ as shown in Figure-3.30. For 
every cell size, the tip radius values fluctuate during the growth. This fluctuation is shown by the red-dashed line in Figure-3.30. The fluctuating of the tip radius increases as the cell size increases, but the fluctuations are almost equal at cell size less than $0.25 \mu \mathrm{m}$.

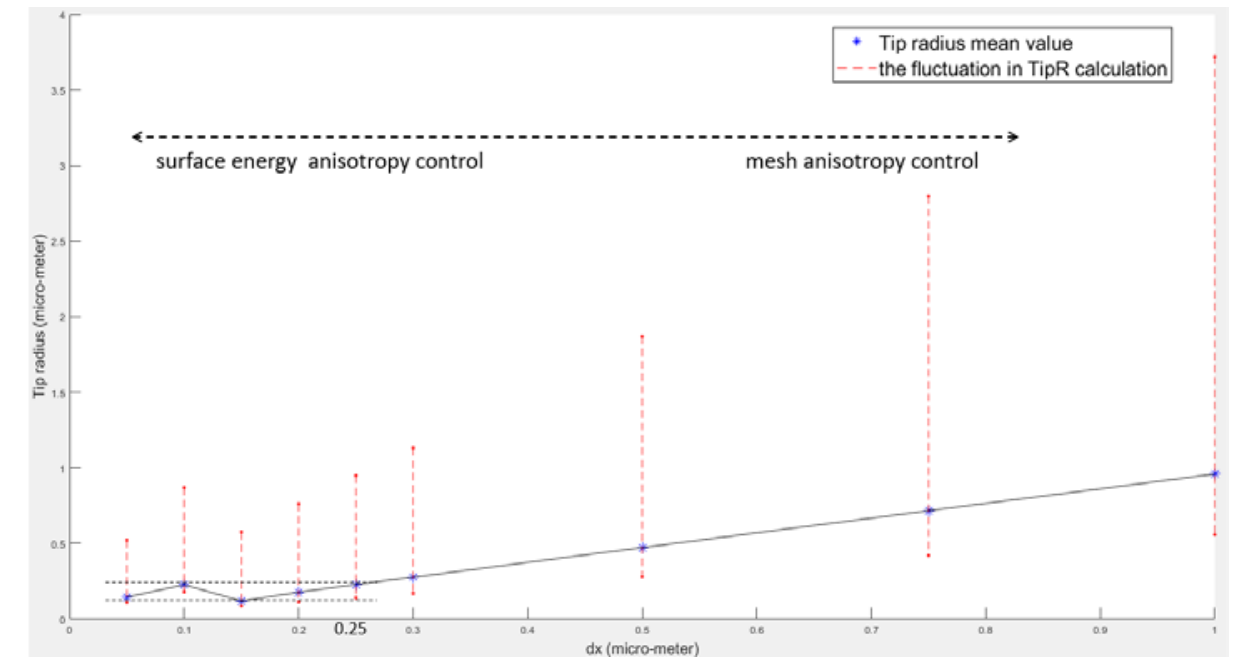

Figure-3.30: The relationship between the tip radius (TipR) and the cell size (dx).

Figure-3.30 shows that at a cell size more than $0.25 \mu \mathrm{m}$, the mesh anisotropy controls the growth because the relation $\mathrm{dx}$-TipR has a proportional relationship. The proportionality indicates that the tip radius is computed from the size of only one cell located on the top of the dendrite, and as the size of this cell increases the tip radius increases. Reversely, at a cell size less than or equal $0.25 \mu \mathrm{m}, \mathrm{dx}$-TipR relationship fluctuates that indicates that there is stronger influence of the surface energy anisotropy and less significant effect from mesh anisotropy. The dendrites produced by simulations with $\mathrm{dx}=0.05 \mu \mathrm{m}, \mathrm{dx}=0.25 \mu \mathrm{m}$, and $\mathrm{dx}=0.5 \mu \mathrm{m}$ are shown in Figure-3.31. 

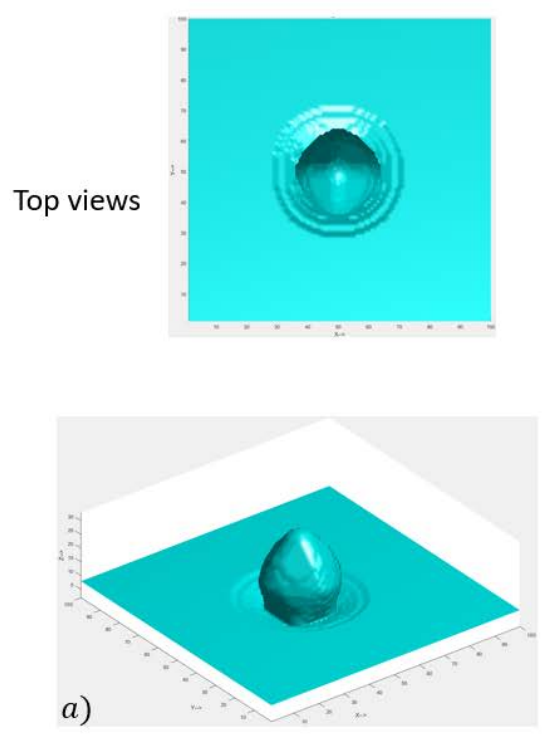

b)
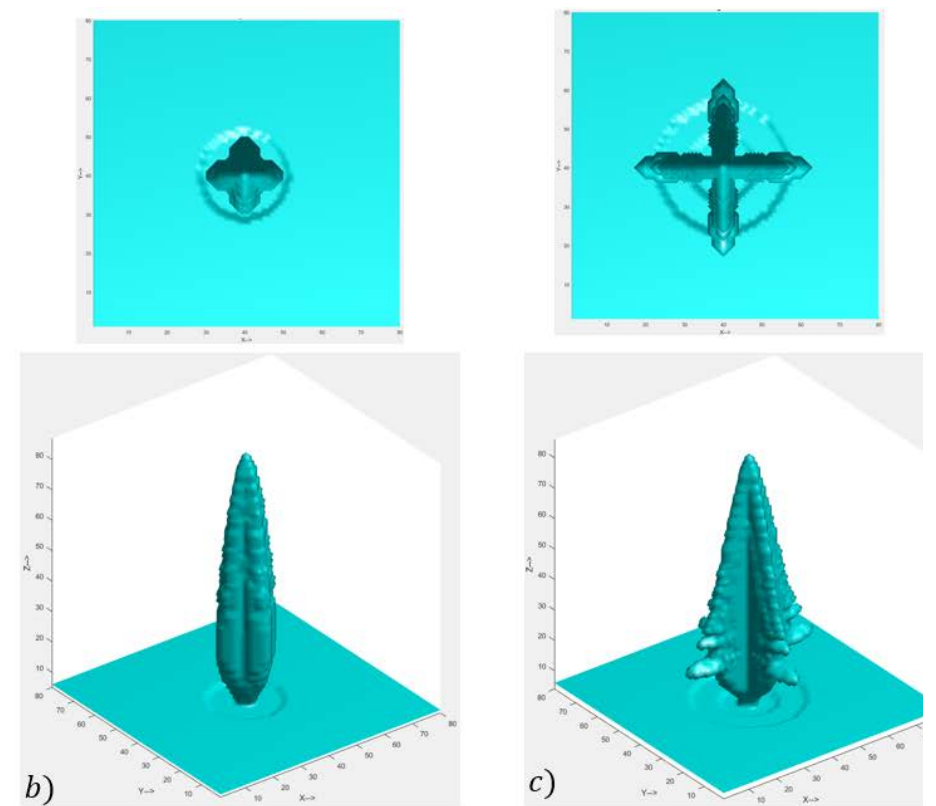

c)

Figure-3.31: The developed dendrite with cell size equal to (a) $0.05 \mu \mathrm{m}$, (b) $0.25 \mu \mathrm{m}$, and (c) $0.5 \mu \mathrm{m}$.

In Figure-3.31a, the produced dendrite tip is more paraboloid with a smooth tip. Figure$3.31 \mathrm{~b}$ shows the developed dendrite with $\mathrm{dx}=0.25 \mu \mathrm{m}$ and growth time was equal to 0.0117 sec. With using a higher value of $\mathrm{dx}$ (Figure-3.31c), some secondary arms start to form at a growth time equal to $0.001 \mathrm{sec}$, as shown in the figure. Since, the used anisotropy coefficient in $\mathrm{xy}$ plane is equal to zero, the growth in $\mathrm{x}$ and $\mathrm{y}$ directions are restricted physically, so the early growth of secondary arms in Figure-3.31c is in particular due to stronger influence of mesh anisotropy. The conclusion is that a cell size $\leq 0.25 \mu \mathrm{m}$ incorporates the effect of the surface energy anisotropy and can be used to capture the physics of growth. 


\section{Chapter-4: CALPHAD-Cellular Automata Coupling Results}

In this chapter the results of the investigations of free and constrained growth of dendritic microstructures are presented and discussed. First, the effects of the surface energy anisotropy and the initial undercooling on the equiaxed growth are investigated. In addition, the growth of a dendrite subjected to different cooling rates is studied. Also, the solidification of a duplex steel with high and low cooling rates is investigated. Second, a series of simulation runs are used to study the constrained growth of dendrites. The effect of curvature calculation method on the results are investigated. In addition, two simulations that include heat diffusion solver will be used to investigate the development of thermal field during the solidification process. The obtained results from simulations include: the solidification morphology, phase fractions, the solutes segregation in solid, the solutes rejection in liquid, and the capillarity undercooling.

To quantify the segregation, we proposed a segregation-index (SIndex) which is equal to the maximum solute concentration in solid $(\max S)$ minus the minimum solute concentration in solid (minS) over the mean solute concentration in solid (meanS) as calculated by Equation-4.1. As the segregation-index value increases, the segregation becomes more severe.

$$
\text { SIndex }=\frac{\max S-\min S}{\text { meanS }} \quad E q 4.1
$$


The developed model can handle the solidification of ferritic and austenitic steels depending on the input of a nominal alloy composition. Table-4 lists some typical composition of stainless steels.

Table-4: Stainless steels compositions. [9]

\begin{tabular}{|c|c|c|c|c|c|}
\hline \multirow[b]{2}{*}{ AISI Type } & \multicolumn{5}{|c|}{ Nominal Composition (\%) } \\
\hline & $\mathrm{C}$ & $\mathrm{Mn}$ & $\mathrm{Cr}$ & $\mathrm{Ni}$ & Other \\
\hline \multicolumn{6}{|c|}{ Austenitic grades } \\
\hline 301 & $0.15 \max$ & 2.0 & $16-18$ & $6-8$ & \\
\hline 302 & $0.15 \max$ & 2.0 & $17-19$ & $8-10$ & \\
\hline 304 & $0.08 \max$ & 2.0 & $18-20$ & $8-12$ & \\
\hline $304 \mathrm{~L}$ & $0.03 \max$ & 2.0 & $18-20$ & $8-12$ & \\
\hline 309 & $0.20 \max$ & 2.0 & $22-24$ & $12-15$ & \\
\hline 310 & $0.25 \max$ & 2.0 & $24-26$ & $19-22$ & \\
\hline 316 & $0.08 \max$ & 2.0 & $16-18$ & $10-14$ & $2-3 \%$ Mo \\
\hline $316 \mathrm{~L}$ & $0.03 \max$ & 2.0 & $16-18$ & $10-14$ & $2-3 \% \mathrm{Mo}$ \\
\hline 321 & $0.08 \max$ & 2.0 & $17-19$ & 9-12 & $(5 \times \% \mathrm{C}) \mathrm{Ti} \min$ \\
\hline 347 & $0.08 \max$ & 2.0 & $17-19$ & $9-13$ & $(10 \times \% \mathrm{C}) \mathrm{Nb}-\mathrm{Ta} \min$ \\
\hline \multicolumn{6}{|c|}{ Martensitic grades } \\
\hline 403 & $0.15 \max$ & 1.0 & $11.5-13$ & & \\
\hline 410 & $0.15 \max$ & 1.0 & $11.5-13$ & & \\
\hline 416 & $0.15 \max$ & 1.2 & $12-14$ & & $0.15 \% \mathrm{~S} \min$ \\
\hline 420 & $0.15 \mathrm{~min}$ & 1.0 & $12-14$ & & \\
\hline 431 & $0.20 \max$ & 1.0 & $15-17$ & $1.2-2.5$ & \\
\hline $440 \mathrm{~A}$ & $0.60-0.75$ & 1.0 & $16-18$ & & $0.75 \%$ Mo $\max$ \\
\hline $440 \mathrm{~B}$ & $0.75-0.95$ & 1.0 & $16-18$ & & $0.75 \%$ Mo $\max$ \\
\hline $440 \mathrm{C}$ & $0.95-1.20$ & 1.0 & $16-18$ & & $0.75 \%$ Mo $\max$ \\
\hline \multicolumn{6}{|c|}{ Ferritic grades } \\
\hline 405 & $0.08 \max$ & 1.0 & $11.5-14.5$ & & $0.1-0.3 \% \mathrm{Al}$ \\
\hline 430 & $0.15 \max$ & 1.0 & 14-18 & & \\
\hline 446 & $0.20 \max$ & 1.5 & $23-27$ & & \\
\hline
\end{tabular}

The nominal alloy composition used in all simulation runs in this chapter varies in the range of $13 \% w t-18 \% w t$ for chromium and $0.05 \% w t-0.15 \% w t$ for carbon. In addition, the diffusion coefficients of carbon and chromium are considered constant and equal to $2 \times 10^{-9} \mathrm{~m}^{2} / \mathrm{s}$.

For all simulation-runs in this chapter (excepting section-4.6 in which the heat equation is solved), the temperature field is uniform within a regular computational domain with a zero 
or constant cooling rate and without a temperature gradient. In addition, the initial solutes concentration of $\mathrm{Cr}$ and $\mathrm{C}$ are equal to the nominal alloy composition and uniformly distributed within the computational domain. As described in section-3.7, the boundary conditions of solving mass diffusion equation are periodical at all side surfaces of the domain and isolated at the top and the bottom. These boundary conditions will ensure the mass conservation and no leak can occur.

\subsection{The Effect of Surface Energy Anisotropy on the Free Growth of Equiaxed Grain}

In this section a study of the effects of anisotropy coefficient (as described by Equation3.7) are investigated. Three different simulation-runs are used with the following parameters for each: isothermal condition with initial temperature $=1505^{\circ} \mathrm{C}$, small initial spherical solid, nominal alloy composition $C r 18 \% w t-C 0.08 \% w t$, and Gibbs-Thomson

coefficient $\Gamma=1^{-7} K \cdot m$. For this alloy at a flat $\mathrm{S} / \mathrm{L}$ interface, the distribution coefficient of carbon $\left(k_{C}^{o}\right)$ is equal to 0.15 and is equal to 0.95 for chromium $\left(k_{C r}^{o}\right)$. The anisotropy coefficient is different for each simulation; The first simulation-run (RUN411) has very small anisotropy coefficients (section-1.7.5) that are equal to 0.002 , the second simulationrun (RUN412) has anisotropy coefficients equal to 0.03 , and the third simulation-run (RUN413) has anisotropy coefficients equal to 0.3 .

\section{RUN411:}

The developing microstructure of simulation RUN411 is shown in Figure-4.1. The initial shape of the solid phase is spherical as illustrated in Figure-4.1a. At a simulation time equal 
to $0.0005 \mathrm{sec}$ (Figure-4.1b), the spherical morphology is maintained because of the weak effect of surface energy anisotropy. In this case, the surface energy is almost isotropic, and the growth rates are equal in all directions due to the equal effect of surface energy. Spherical shape is maintained, even though the mesh anisotropy is present, because mesh with a high resolution (cell size $\mathrm{dx}=0.2 \mu \mathrm{m}$ ) was used. However, the spherical morphology starts to be unstable as the solid fraction increases (Figure-4.1c) and perturbations along the preferred growth directions are produced. For a growth with zero anisotropy, the spherical stability is expected to be broken and produced a coral like shape as shown in Figure-1.11b.
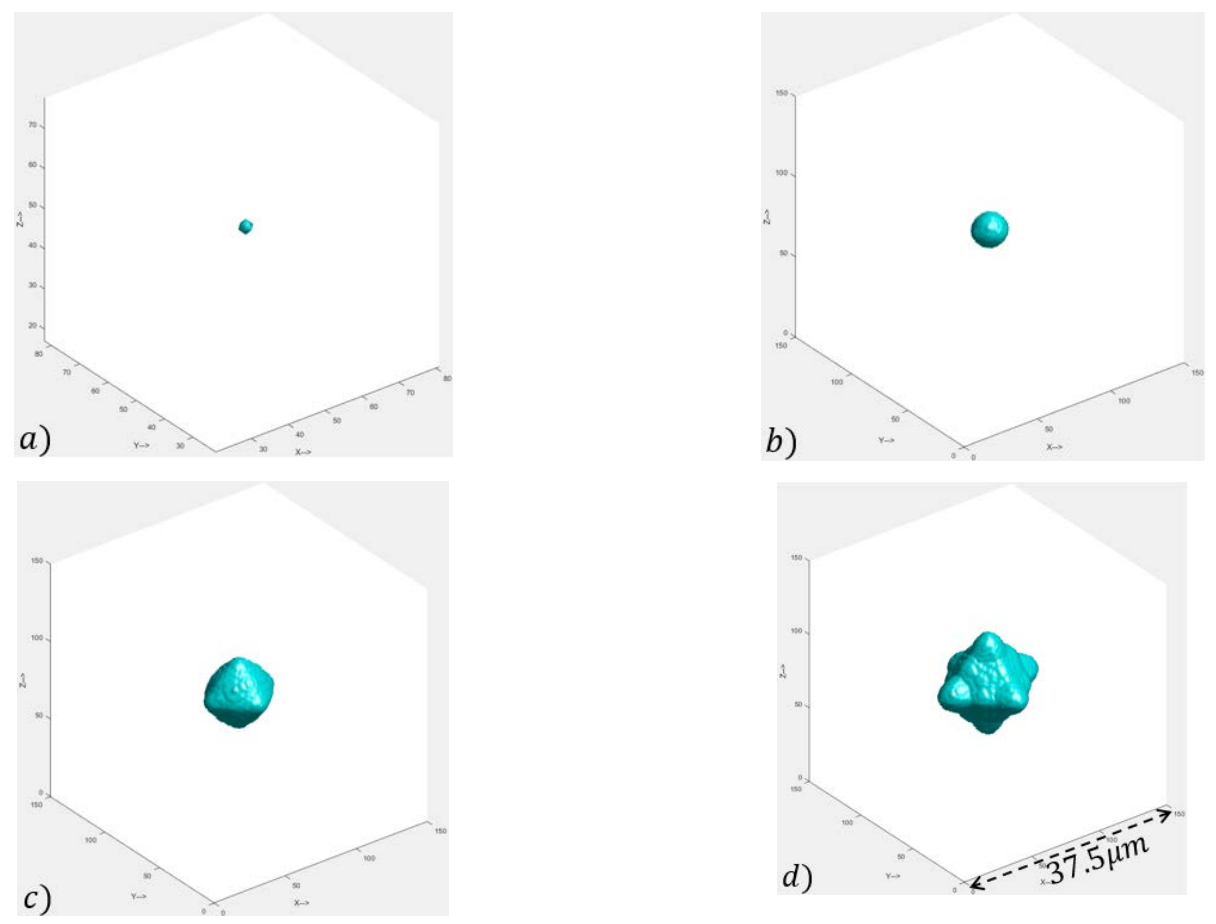

Figure-4.1: Unconstrained dendrite growth with very low anisotropy coefficients (anis $=0.02$ ). (a) initial solid fraction, (b) solid fraction at $\mathrm{t}=0.0005 \mathrm{sec}$, (c) solid fraction at $\mathrm{t}=0.00175 \mathrm{sec},(\mathrm{d})$ solid fraction at $\mathrm{t}=0.0025 \mathrm{sec}$. [RUN411] 
Figure-4.1d shows a manifestation of the growth along the preferred growth directions. Eventually, after a longer time of the simulation, an equiaxed dendrite is expected to form. Figure-4.2 shows the concentration of carbon in liquid surrounding the growing solid during the simulation. At an early stage of the simulation (Figure-4.2a), the rejected solutes form spherical layers around the growing spherical solid. Since the solute diffusion in liquid is slow, the solute removal from the $\mathrm{S} / \mathrm{L}$ interface is slow and hence the growth rate. As the solid fraction increases and the spherical morphology is broken (Figure-4.2b), the surface area of the solid increases and so the diffusion process becomes faster.
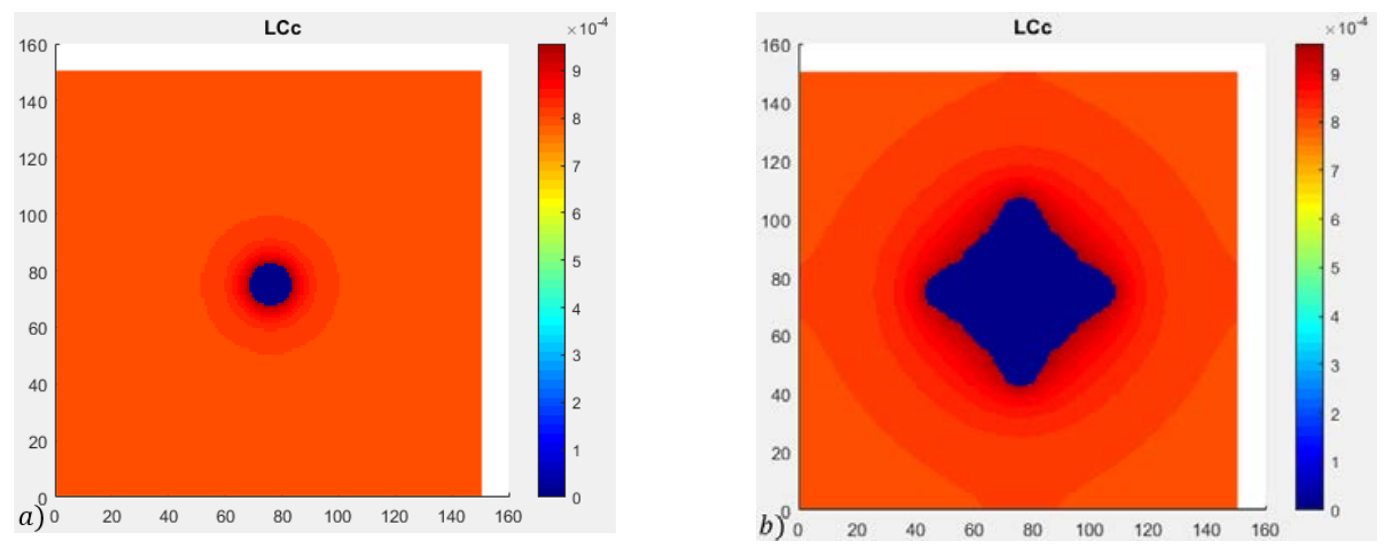

Figure-4.2: (a) the solute (carbon) distribution in liquid at $\mathrm{t}=0.0005 \mathrm{sec}$, (b) the solute (carbon) distribution in liquid at $\mathrm{t}=0.0025 \mathrm{sec}$.

\section{RUN412:}

Metals with BCC and FCC crystal structure solidify from bulk liquid with six preferred $<100>$ growth directions producing equiaxed microstructure morphology under unconstrained growth conditions. The effect of preferred growth directions can be included in the simulation using anisotropy coefficients in Equation-3.7. The resulting 
microstructure of RUN412 with anisotropy coefficients equal to 0.03 is shown in Figure4.3. In contrast to Figure-4.1d, the microstructure is equiaxed (Figure-4.3d) with six primary arms because the maximum growth rate is aligned with the six easy growth directions.
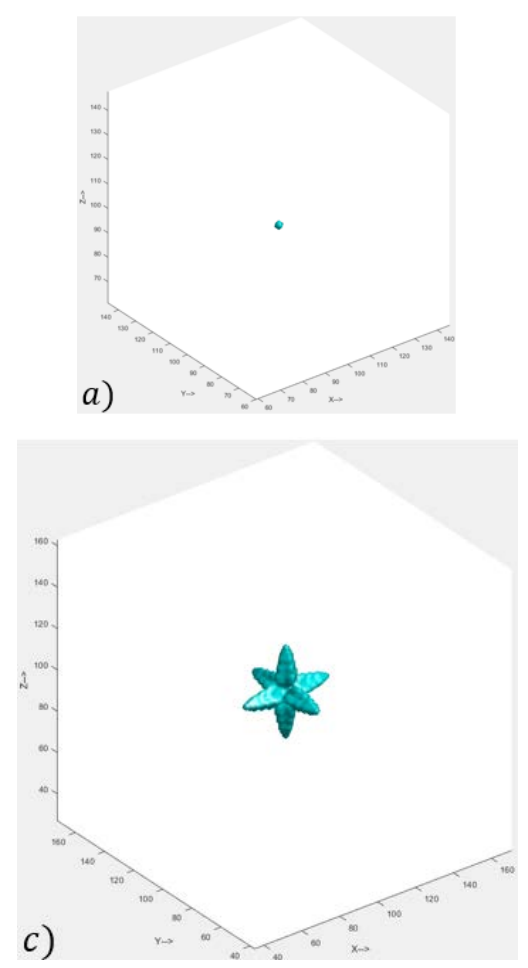
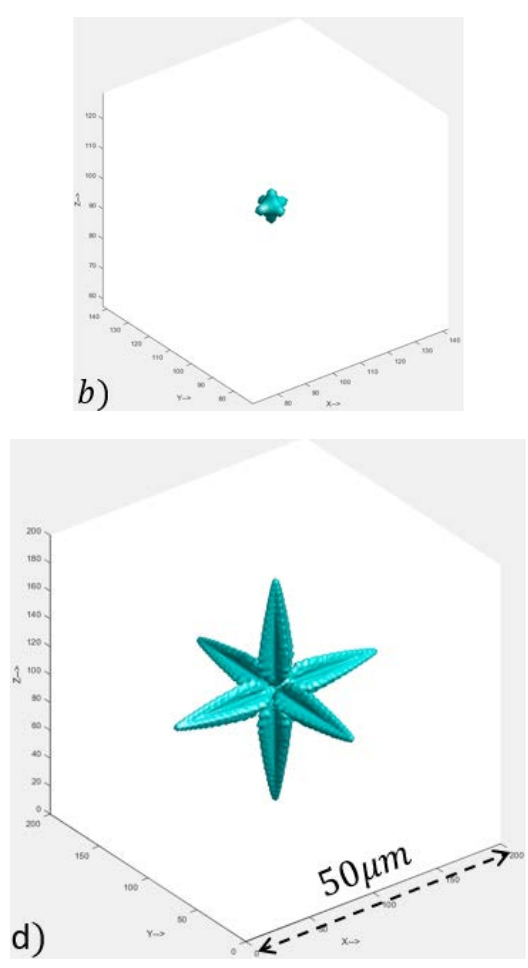

Figure-4.3: (a) the initial grain nucleus, (b) the grain at $\mathrm{t}=0.00039 \mathrm{sec}$, (c) the grain at $\mathrm{t}=0.0027 \mathrm{sec},(\mathrm{d})$ the grain at $\mathrm{t}=0.0093 \mathrm{sec}$. [RUN412]

Numerically, the maximum growth rate along the preferred growth directions are enforced by the anisotropy function (Equation-3.7) which changes the values of the surface energy along the dendrite surface, and the maximum surface energy will be located at the tips of the dendrite. Physically, more atoms will be attached to the tips of the equiaxed dendrite so that the growth rates will be faster there. 

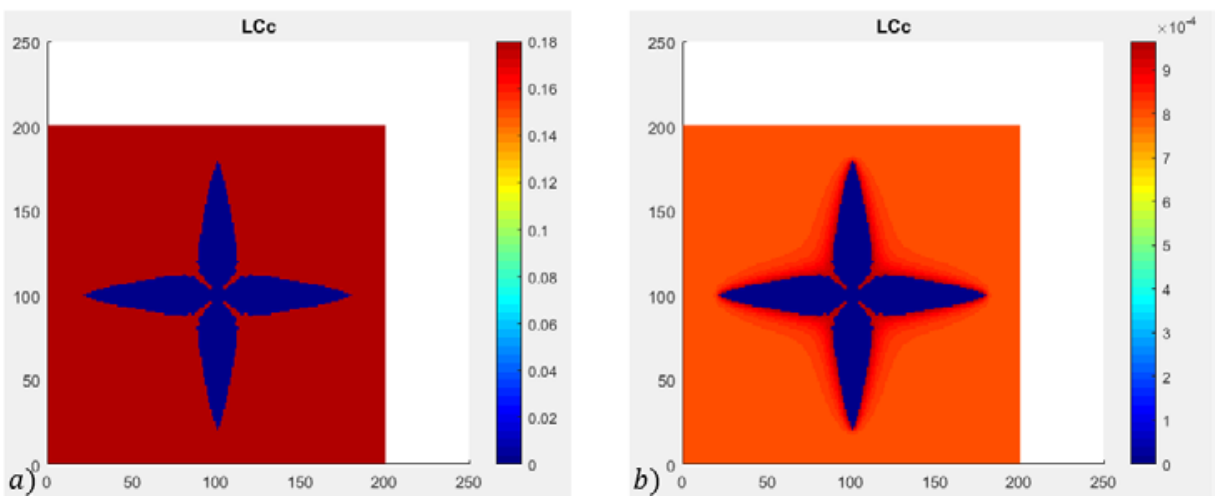

Figure-4.4: (a) the chromium distribution in liquid, and (b) the carbon distribution in liquid. [RUN412]

Figure-4.4 shows the distribution of solutes $(\mathrm{Cr}$ and $\mathrm{C})$ in liquid during the solidification. The distribution of $\mathrm{Cr}$ in the solid is almost equal to the distribution of it in the surrounding liquid (Figure-4.4a), because the distribution coefficient is almost equal to one $\left(k_{C r}^{o}=\right.$ 0.95). In contrast, the distribution of $\mathrm{C}$ in liquid (Figure-4.4b) gradually builds layers of solute around the growing solid phase, because the distribution coefficient is small $\left(k_{C}^{o}=\right.$ 0.15). Since the $\mathrm{S} / \mathrm{L}$ interface of an equiaxed dendrite is larger than the spherical morphology interface, the process of solutes removal from the $\mathrm{S} / \mathrm{L}$ interface is faster and so the growth rate. The pattern of solute distribution around the solid phase is a common result feature that results from the solidification simulation as shown in Figure-4.5.
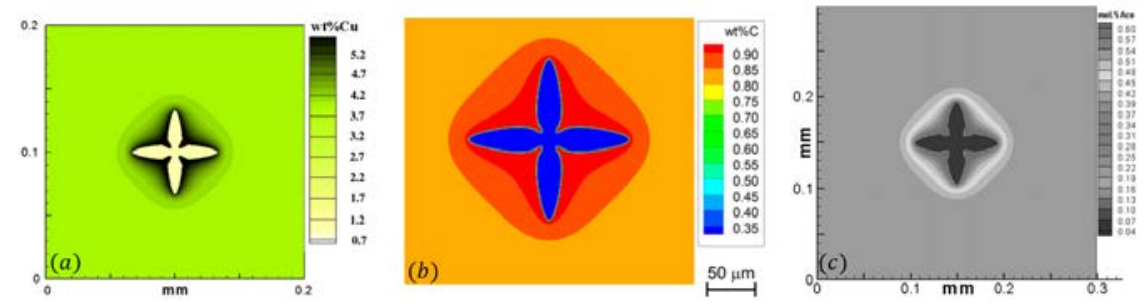

Figure-4.5: The solute layer rejected in liquid as obtained by a) [66], b) [67], and c) [65]. 
Figure-4.6 shows the distribution of the curvature and the capillarity undercooling of the equiaxed dendrite during the simulation. In Figure-4.6a, the positive values of curvature are calculated for the convex interface (e.g. the tips) and the negative values are calculated for the concave interface. Accordingly, the values of capillarity undercooling $\mathrm{d} \operatorname{Tr}$ (Figure4.6b) are calculated using Equation-3.6 and have positive values at convex interface portions and negative values at concave interface portions.
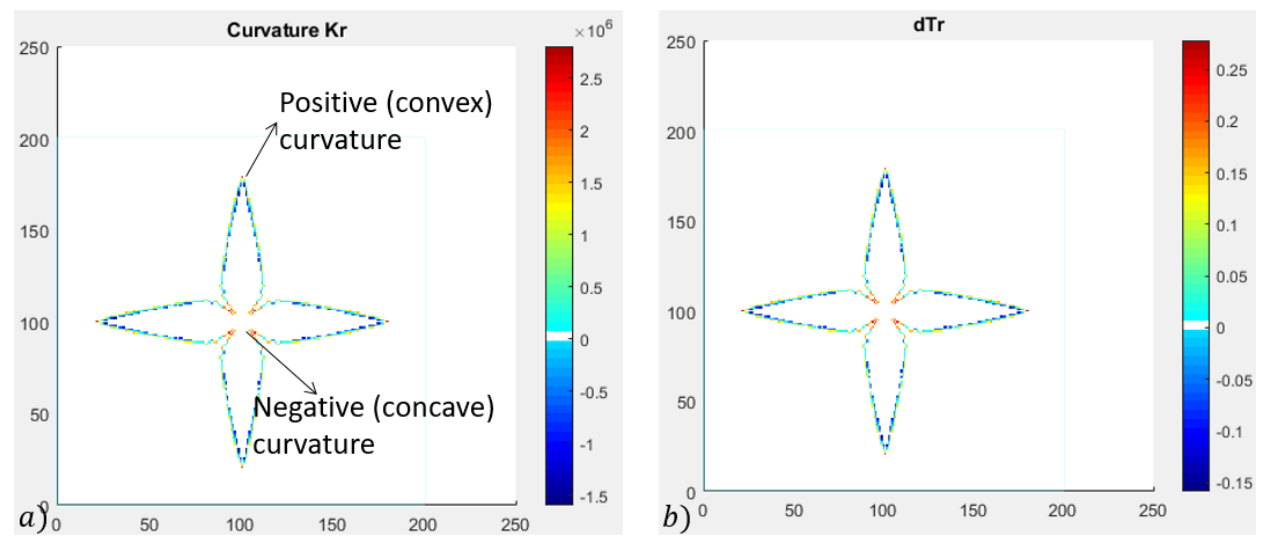

Figure-4.6: A cross section of the dendrite showing (a) the curvature of the S/L interface, and (b) the capillarity undercooling. [RUN412]

The maximum value of $\mathrm{d} \operatorname{Tr}$ is around $0.26^{\circ} \mathrm{C}$ and the minimum value is $-0.15^{\circ} \mathrm{C}$. At convex portions of the $\mathrm{S} / \mathrm{L}$ interface, the melting temperature of solid decreases by the corresponding $\mathrm{d} \operatorname{Tr}$, and, unlikely, the melting temperature of solid increases by $\mathrm{d} \operatorname{Tr}$ at concave portions. The incorporation of $\mathrm{dTr}$ in the calculation of solid fraction is described in section-3.3.5 and Figure-3.13. 


\section{RUN413:}

The third simulation-run (RUN413) uses the same simulation parameters as RUN412 but with a higher value of anisotropy coefficients equal to 0.3 .
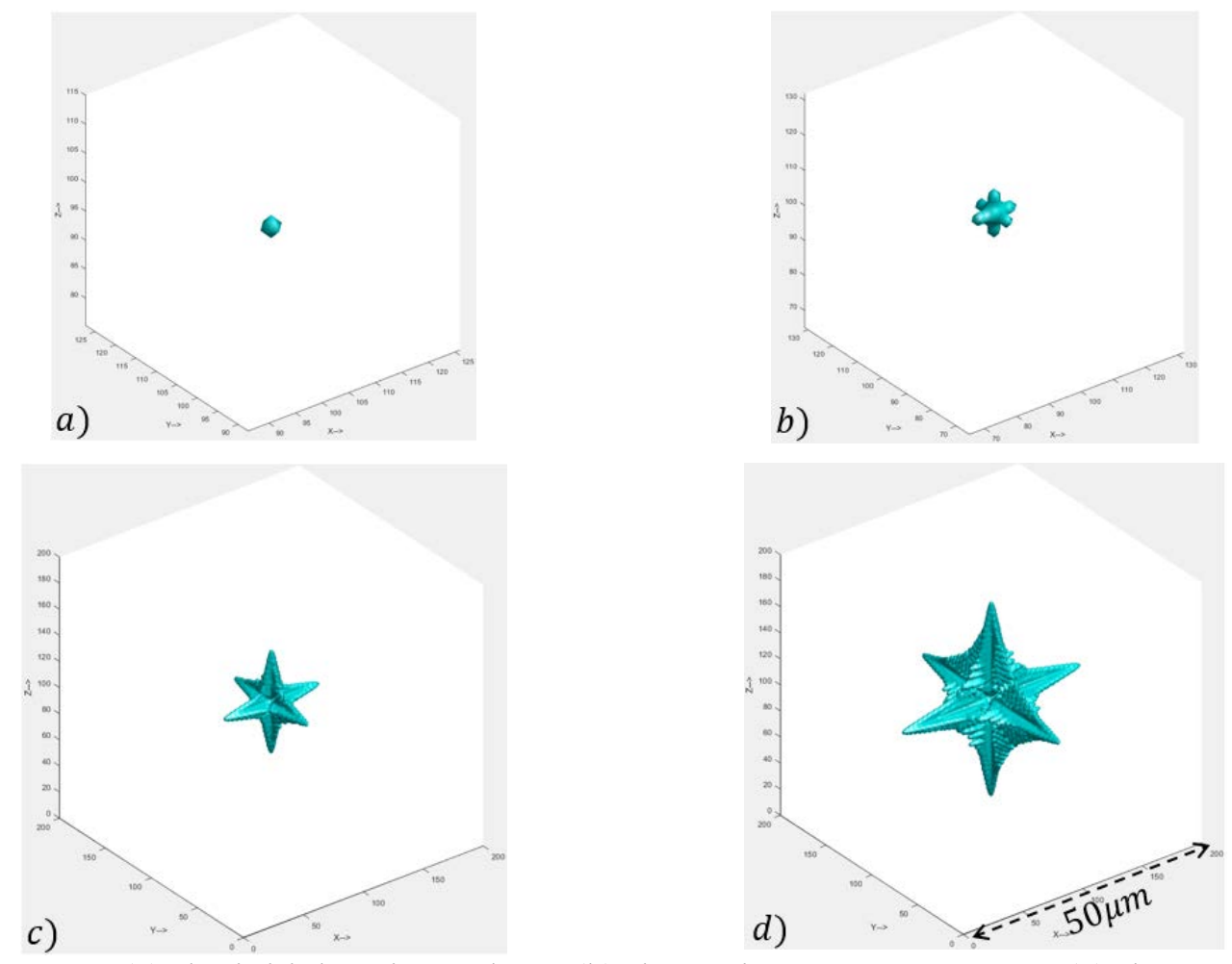

Figure-4.7: (a) the initial grain nucleus, (b) the grain at $t=0.00039 \mathrm{sec}$, (c) the grain at $\mathrm{t}=0.0039 \mathrm{sec},(\mathrm{d})$ the grain at $\mathrm{t}=0.0079 \mathrm{sec}$. [RUN413]

The obtained dendrite morphology of the high anisotropy case (Figure-4.7d) is more fractal than the low anisotropy case (Figure-4.3d). This is expected because higher anisotropy coefficients promote the growth rate along the easy growth directions, which produces more fractal shape. 
In Figure-4.8a a cross section through the dendrite shows the solid fraction distribution. The interfacial cells are in the mushy state, so their solid fractions are less than one. Figure$4.8 \mathrm{~b}$ shows the carbon distribution in solid of RUN413.
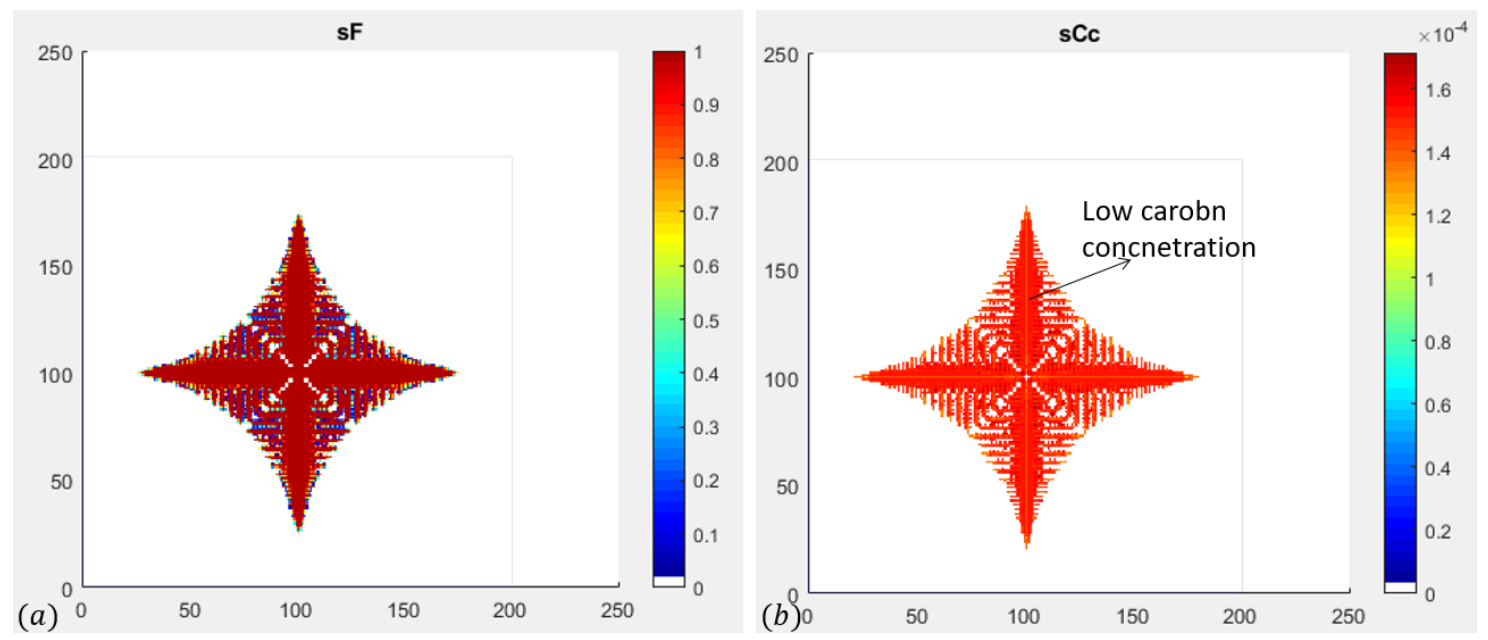

Figure-4.8: (a) a cross section of the solid fraction at $\mathrm{t}=0.0079 \mathrm{sec}$, and (b) the carbon distribution in solid. [RUN413]

The carbon segregation is more pronounced than the chromium segregation because its distribution coefficient is lower. Carbon segregates more on the outer surface of the dendrite, and its concentration is less in the core. The reason of the solute segregation pattern is that at a tip the curvature is large and so the solute concentration in liquid is low. Accordingly, less solute concentration in solid is located along the trace of the tip growth. The segregation-index of carbon $($ SIndex_C) is equal to 0.276 , and the segregation-index of chromium (SIndex_Cr) is equal to 0.001. As expected, the segregation of carbon in solid is much higher than the segregation of chromium since the distribution coefficient of carbon is smaller. 
For comparing RUN412 and RUN413, the segregation index is calculated for RUN412, and it is found that SIndex_C $=0.2322$ and SIndex_Cr $=0.0004$. In addition, the growth time for RUN412 was equal to $0.009 \mathrm{sec}$ and it was equal to $0.007 \mathrm{sec}$ for RUN413. RUN412 has a lower anisotropy coefficient, so the growth rate is slower and the solute segregation is less severing. In addition, the morphology of RUN412 (Figure-4.3d) is smoother than RUN413 (Figure-4.7d), because a smaller anisotropy promotes the effect of capillarity undercooling which tends to flatten the $\mathrm{S} / \mathrm{L}$ interface.

\subsection{The Effect of Undercooling on the Free Growth of Equiaxed Grain}

Increasing undercooling increases the driving force of solidification as described in section-1.7.1. Solidification undercooling affects the growth velocity, the dendrite morphology, and the solute segregation in solid. Two simulation-runs are used to investigate the effect of undercooling with the following parameters: nominal alloy composition $13 \mathrm{wt} \%$ of $\mathrm{Cr}$ and $0.15 \mathrm{wt} \%$ of $\mathrm{C}$, Gibbs-Thomson coefficient $\Gamma=1^{-7} \mathrm{~K} \cdot \mathrm{m}$, and anisotropy coefficient equal to 0.2. However, the initial temperature of the first simulation-run (RUN421) is equal to $1500{ }^{\circ} \mathrm{C}$ with $2{ }^{\circ} \mathrm{C}$ undercooling, and the initial temperature of the second simulation-run (RUN422) is equal to $1496{ }^{\circ} \mathrm{C}$ with $6{ }^{\circ} \mathrm{C}$ undercooling. It is expected that the developed microstructure of RUN422 should be more fractal with more solutes segregation because of the higher undercooling. 

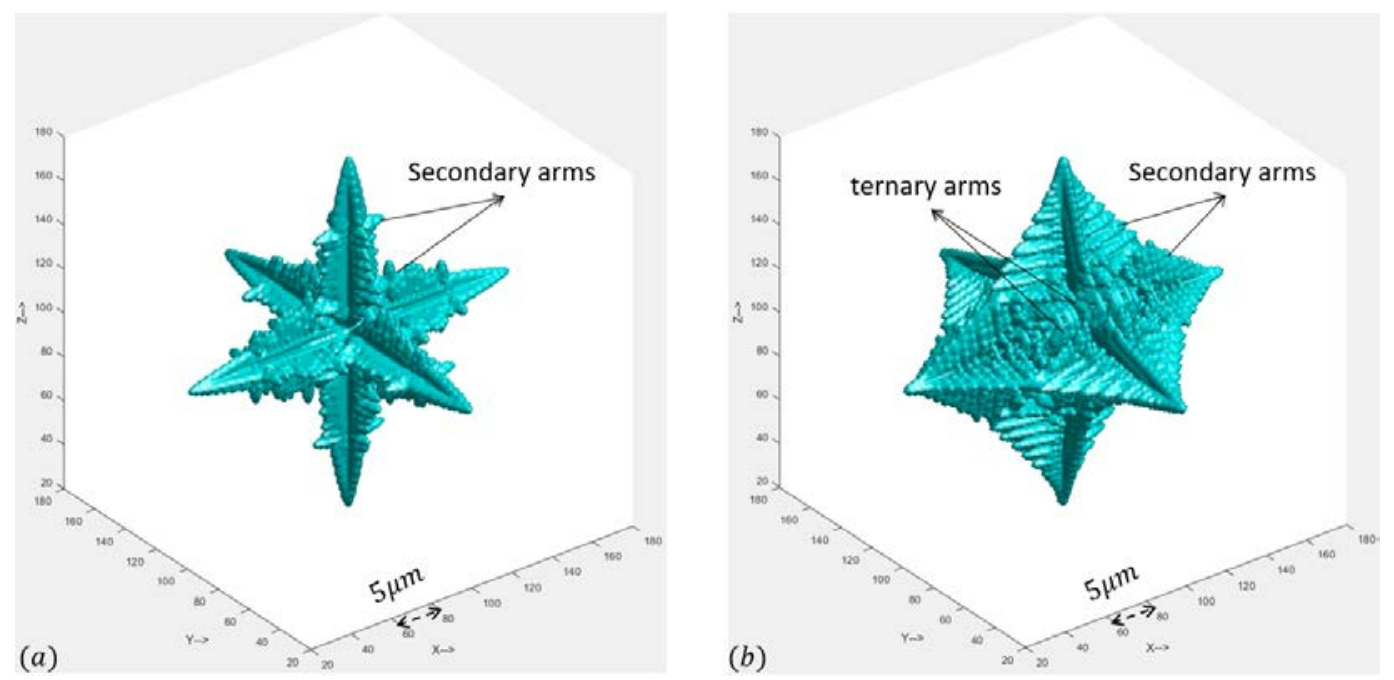

Figure-4.9: (a) the developed dendrite of RUN421, and (b) the developed dendrite of RUN422.

The developed microstructure of RUN421 is shown in Figure-4.9a. The dendrite is an equiaxed with six primary arms and some secondary arms. Figure- $4.9 \mathrm{~b}$ shows the simulation-run RUN422 which has the same parameters as RUN421 but with a higher undercooling. The developed microstructure in RUN422 is more fractal with long secondary arms and some short tertiary arms. The solidification time of RUN421 was equal to 0.043 and the solidification time of RUN422 was equal to $0.0133 \mathrm{sec}$. The microstructure is more dendritic and the growth rate is higher in the case of a higher undercooling because of the higher driving force of solidification. Figure-4.10 shows a cross section of the developed microstructure of RUN421 and RUN422. For RUN421, the segregation indices are SIndex_C $=0.2$ and SIndex_Cr $=0.0029$, and for RUN422, they are SIndex_C $=$ 0.3277 and SIndex_Cr $=0.0041$. In the case of the higher undercooling, the solutes segregate more at the boundary of the microstructure. 

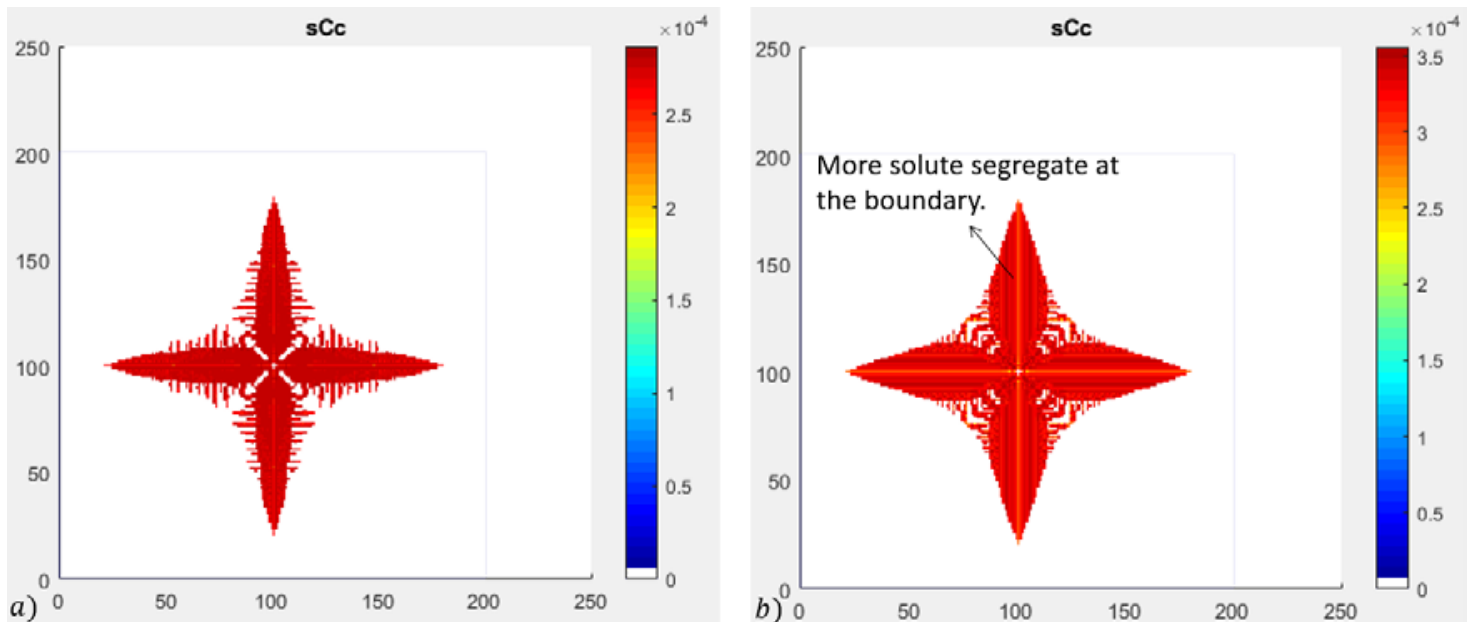

Figure-4.10: (a) a cross section of the developed microstructure of RUN421 (low undercooling), and (b) a cross section of the developed microstructure of RUN422 (high undercooling).

As a result, the growth of a dendrite with a higher undercooling is faster than a dendrite with a lower undercooling. Similarly, the solutes segregation of a solidification with a higher undercooling is more severe than the solidification with a lower undercooling. The reason is that more undercooling increases the driving force of the solidification, and the microstructure deviates more from the equilibrium composition.

\subsection{The effect of cooling rate}

Increasing cooling rate increases the growth rate and produces more fractal microstructure. In addition, increasing cooling rate deviates the composition of microstructure more from the equilibrium that increases the segregation. The model is used to predict the effect of different cooling rates. Four different simulation-runs with the same parameters and different cooling rates are investigated in this section. The used simulation parameters are 
nominal alloy composition $16 \mathrm{wt} \%$ of $\mathrm{Cr}$ and $0.08 \mathrm{wt} \%$ of $\mathrm{C}$, initial temperature $=1504{ }^{\circ} \mathrm{C}$, Gibbs-Thomson coefficient $\Gamma=1^{-7} K \cdot m$, and anisotropy coefficient equal to 0.2 . Different cooling rates are used for each run where RUN431 has cooling rate equal to 50 ${ }^{\circ} \mathrm{C} / \mathrm{s}$, RUN432 has cooling rate equal to $100{ }^{\circ} \mathrm{C} / \mathrm{s}$, RUN433 has cooling rate equal to 200 ${ }^{\circ} \mathrm{C} / \mathrm{s}$, and RUN434 has cooling rate equal to $500{ }^{\circ} \mathrm{C} / \mathrm{s}$. Their developed microstructures are shown in Figure-4.11.
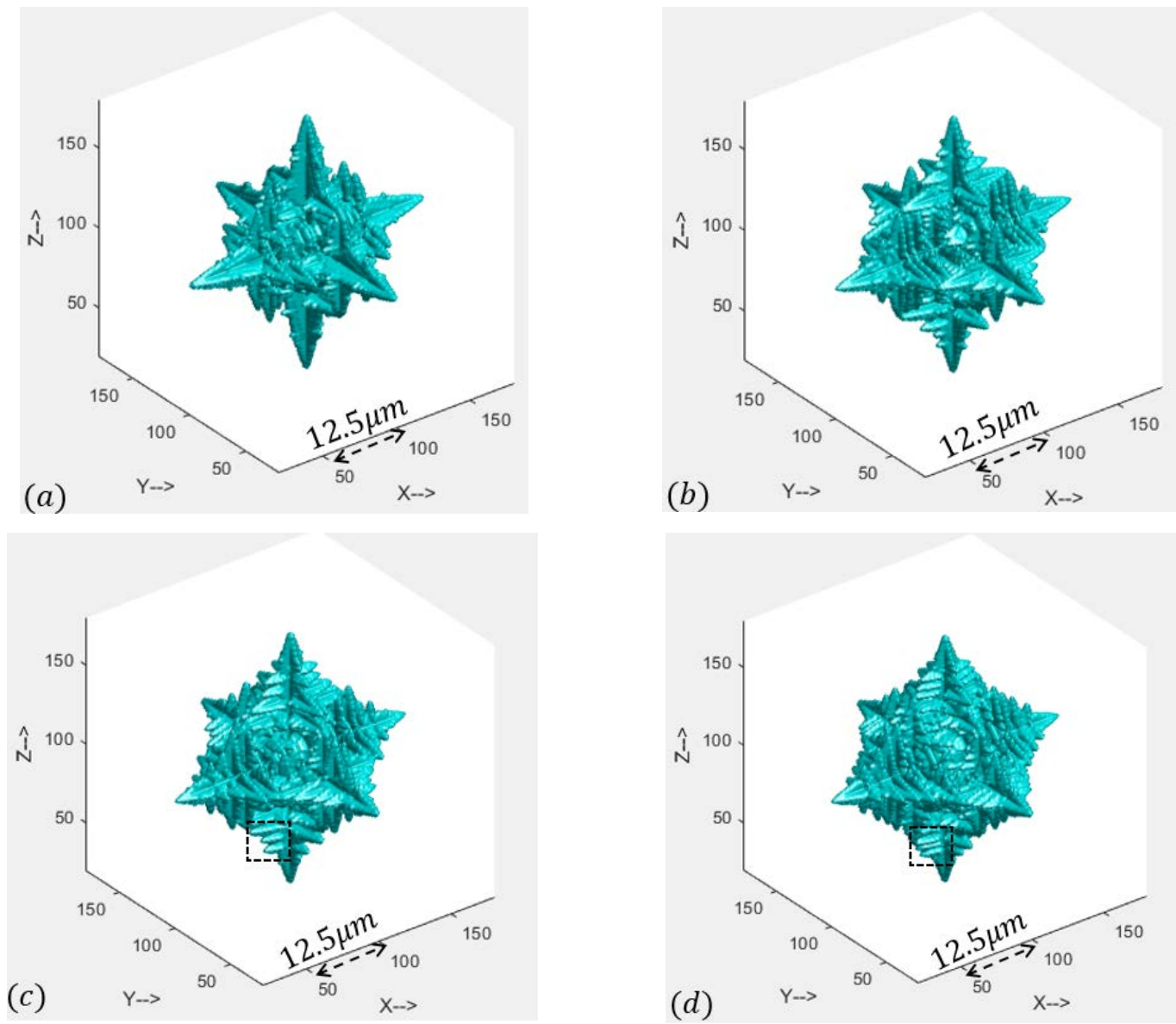

Figure-4.11: The microstructure morphology RUN433, and (d) RUN434. 
The solidification time and solute segregations of simulation-runs shown in Figure-4.11 are listed below:

- RUN431: solidification time $=0.0061 \mathrm{sec}$, SIndex_C $=0.35$, SIndex_Cr $=0.0017$.

- RUN432: solidification time $=0.0060 \mathrm{sec}$, SIndex_C $=0.38$, SIndex_Cr $=0.0017$.

- RUN433: solidification time $=0.0057 \mathrm{sec}$, SIndex_C $=0.4$, SIndex_Cr $=0.0019$.

- RUN434: solidification time $=0.0050 \mathrm{sec}$, SIndex_C $=0.47$, SIndex_Cr $=0.0021$.

The results presented in Figure-4.11 show that as the cooling rate increases the microstructure becomes finer and more dendritic, forming more secondary and tertiary arms. For example, comparing the window-box in Figure-4.11d and 4.10c, the secondary arms spacing in the case of the higher cooling rate (Figure-4.11d) is smaller than the case of the lower cooling rate (Figure-4.11c). This indicates that the microstructure in Figure4.11d is finer than the one in Figure-4.11c, as described in section-1.7. In addition, the solutes segregation increases as the cooling rate increases, as estimated by the segregation indices.

\subsection{Solidification of Duplex Steels}

The microstructures of duplex stainless steels consist of austenitic and ferritic phases. They have good as-welded mechanical properties and excellent corrosion resistance properties [68]. In order to obtain a duplex microstructure, the nominal alloy composition should be located in the tie-triangle area as shown in Figure-4.12a. Two simplifications are 
considered in this study; The zone of FCC-BCC (Figure4.12a) is ignored, and the tietriangle is divided into two zones by a boundary line, as shown in Figure-4.12b. Thus, if a state is initially located to the right of the boundary line is considered to be in the L-BCC zone, and the state is in the L-FCC-BCC zone if it is located to the left of the boundary line. Using CALPHAD allows the algorithm to predict the solidifying phases, which can be liquid-ferrite or liquid-austenite. This simplification is physically reasonable since the nucleation needs some undercooling, and it is considered that crossing the boundary line provides the required undercooling for nucleation.
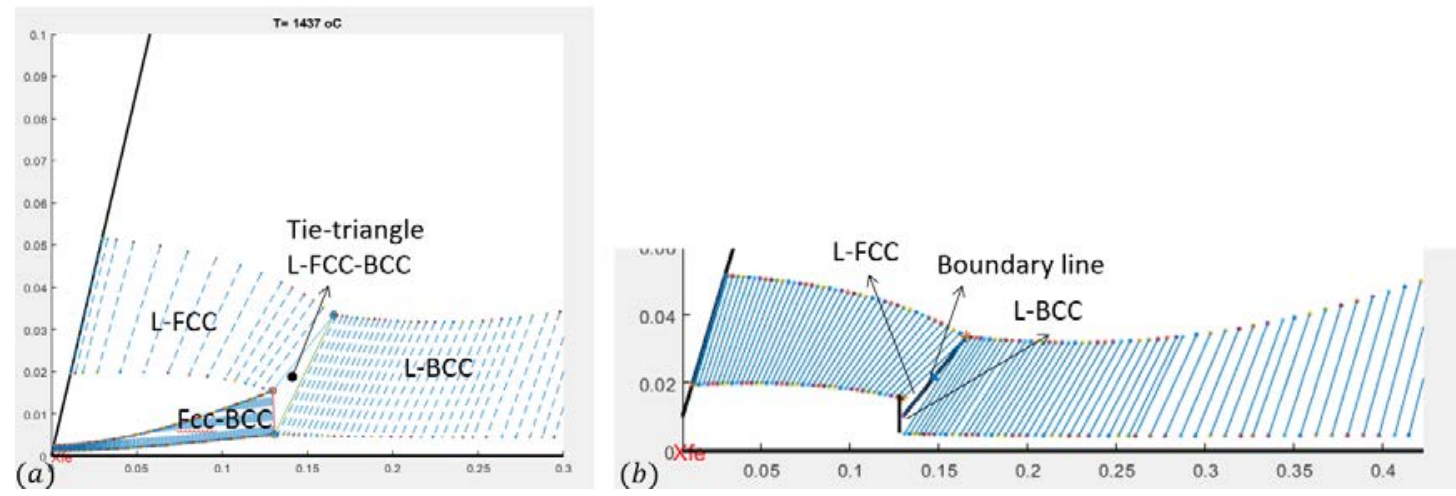

Figure-4.12: A simplifying assumption for isotherms.

To investigate the solidification of a duplex microstructure, an alloy with initial composition $\mathrm{Cr} 8 \% \mathrm{wt}-\mathrm{C} 0.5 \% \mathrm{wt}$ is used. According to the used thermodynamics database, the state of the alloy is $\mathrm{L}-\mathrm{BCC}$ at $\mathrm{T}=1469.5^{\circ} \mathrm{C}$ and $\mathrm{L}-\mathrm{FCC}$ at $\mathrm{T}=1469.1^{\circ} \mathrm{C}$, as shown in Figure-4.13. Two simulation-runs with different cooling rates are used for this study: RUN441 with $10^{\circ} \mathrm{C} / \mathrm{s}$ cooling rate and RUN442 with $100^{\circ} \mathrm{C} / \mathrm{s}$ cooling rate. 

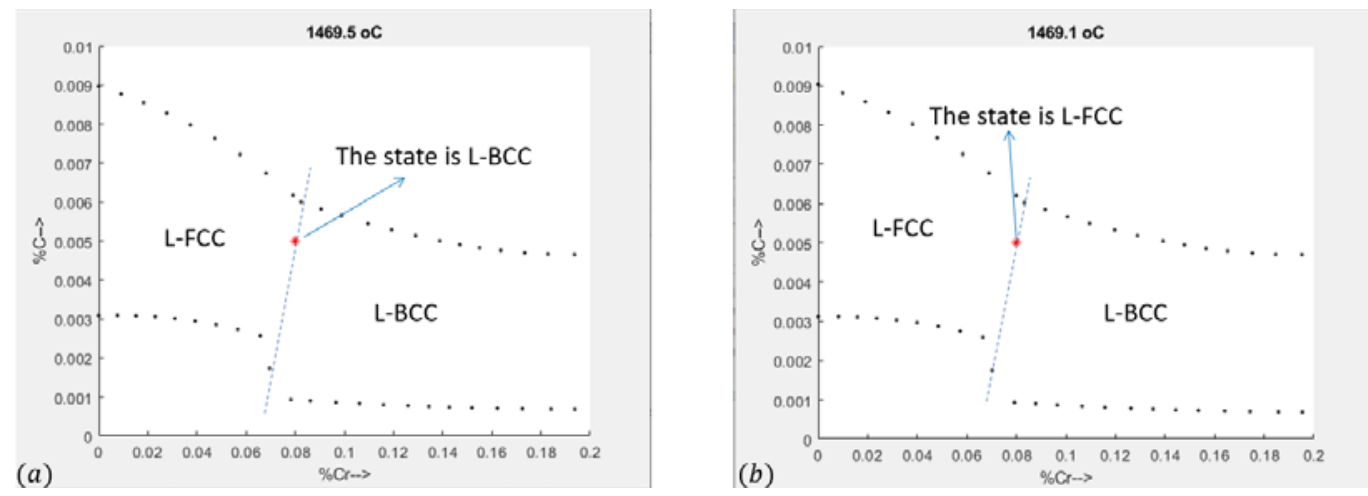

Figure-4.13: For (Cr $8 \% \mathrm{wt}-\mathrm{C} 0.5 \% \mathrm{wt})$ alloy, the thermodynamics state is (a) L-BCC, and (b) L-FCC.

Figure-4.14 shows the growing microstructure of RUN441, and the simulation estimates that the fraction of solid is $96 \%$ ferrite and $4 \%$ austenite. The developed microstructure is almost ferritic with some trace of austenite phase at the diagonal directions of the dendrite. Some cells at the dendrite diagonals have high and concave curvatures which cool down the solid and favor the austenite phase thermodynamically.
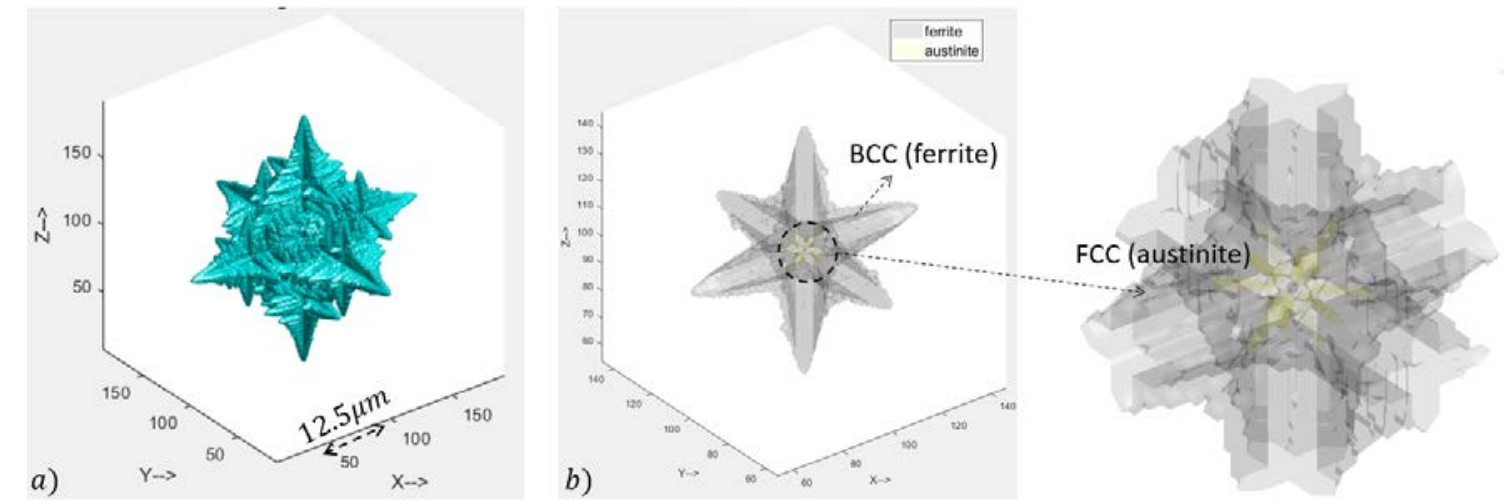

Figure-4.14: The duplex microstructure cooling rate $=10^{\circ} \mathrm{C} / \mathrm{s}$. [RUN441]

Simulation-run RUN422 has the same simulation parameters as RUN421 but with a higher cooling rate that is equal to $100{ }^{\circ} \mathrm{C} / \mathrm{s}$. Figure-4.15 shows the developed duplex 
microstructure, and it is found that the developed microstructure is around $4.1 \%$ ferrite and $95.9 \%$ austenite.
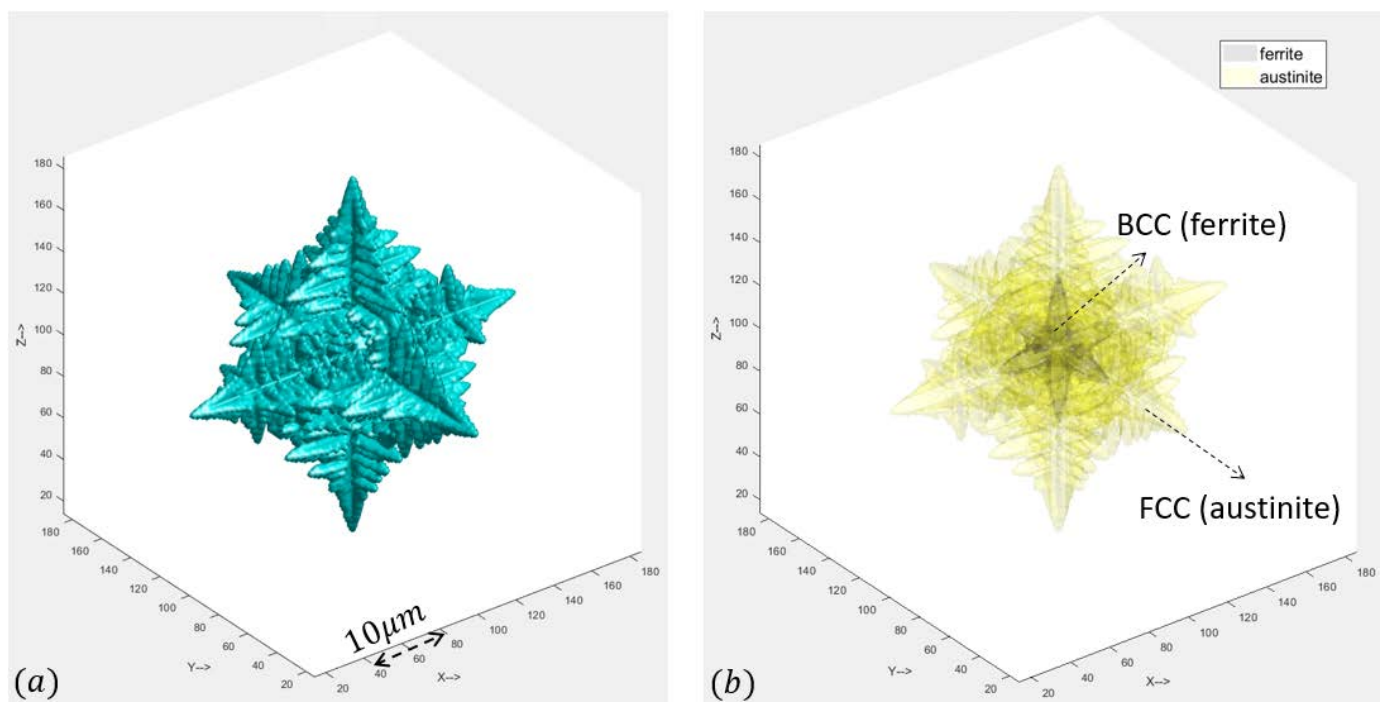

Figure-4.15: The duplex microstructure cooling rate $=100^{\circ} \mathrm{C} / \mathrm{s}$. [RUN442]

In Figure-4.15 the core of the developed microstructure is ferrite because the initial alloy composition was located in the L-BCC zone (Figure-4.13a). As the temperature decreases, austenite becomes more stable and starts to form. Therefore, for an alloy with a nominal composition of $\mathrm{Cr} 8 \% \mathrm{wt}-\mathrm{C} 0.5 \% \mathrm{wt}$, a higher cooling rate produces more austenite in the final duplex microstructure. This is because with the higher cooling rate, the thermodynamics state will cross the boundary line between L-FCC and L-BCC faster that produces more austenitic phase. It is expected that the fraction of austenite phase will increase if the simulation time is extended. Figure-4.16 shows the carbon distribution in solid for a duplex microstructure with low cooling rate and high cooling rate. For RUN441 (Figure-4.16a) the segregation indices are SIndex_C $=0.286$ and SIndex_Cr $=0.0141$. 
Similarly, for RUN442 (Figure-4.16b), the segregation indices are SIndex_C $=0.349$ and SIndex_Cr $=0.0113$.
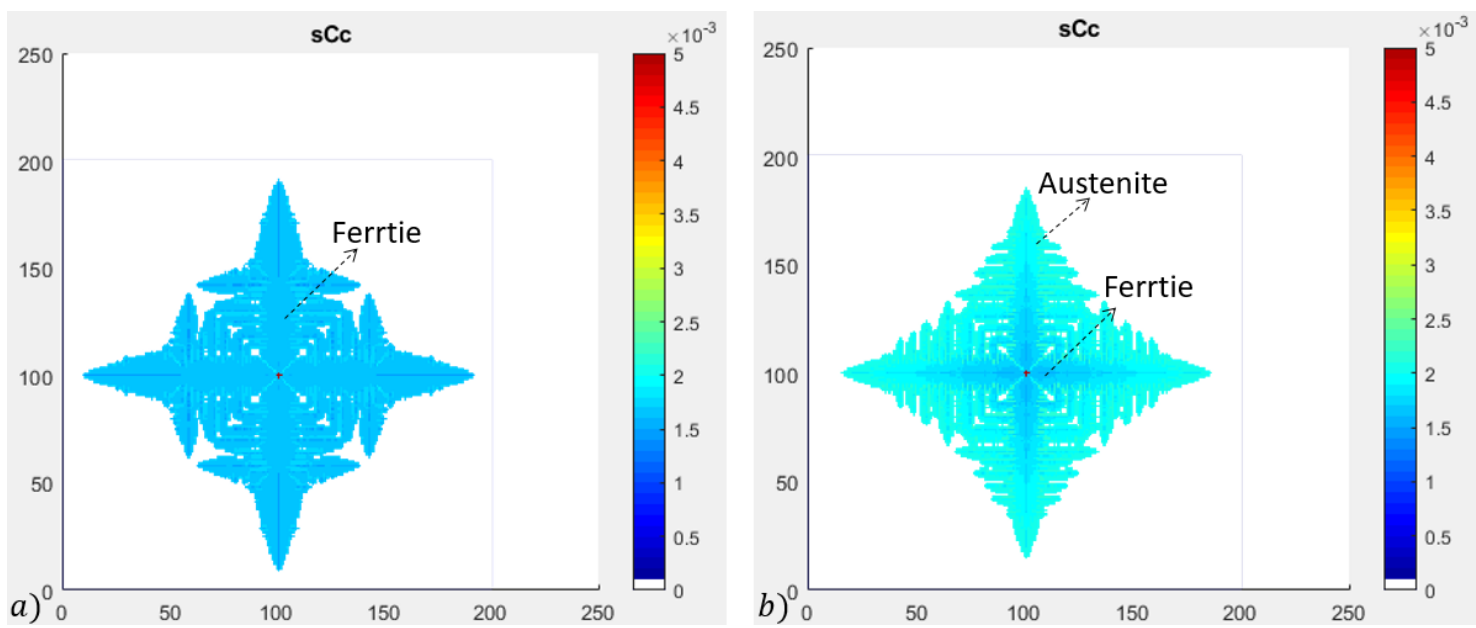

Figure-4.16: The carbon distribution in solid for (a) $10{ }^{\circ} \mathrm{C} / \mathrm{s}$ cooling rate [RUN441] and (b) $100{ }^{\circ} \mathrm{C} / \mathrm{s}$ cooling rate [RUN442].

The solutes segregation in solid of RUN442 is higher than the segregation of RUN441, because RUN442 has a higher cooling rate and produces more austenite fraction. The developed microstructure in Figure-4.16b is a duplex one in which the core is ferritic phase surrounded by austenitic phase.

\subsection{The Effect of Curvature Calculation Models on a Microstructure Evolution}

This section investigates the constrained dendritic growth. Four simulations are investigated all of which have the same parameters (i.e. nominal alloy composition $18 \mathrm{wt} \%$ of $\mathrm{Cr}$ and $0.08 \mathrm{wt} \%$ of $\mathrm{C}$, initial temperature $=1504{ }^{\circ} \mathrm{C}$, cooling rate $=50{ }^{\circ} \mathrm{C} / \mathrm{s}$, GibbsThomson coefficient $\Gamma=1^{-7} K \cdot m$ ) but different curvature calculation model. The first simulation-run (RUN451) uses the cell-count method (Equation-3.21), the second 
simulation-run (RUN452) uses the level-set method with averaging of the solid fractions (Equation-3.4), the third simulation-run (RUN453) uses the level-set method with the optimum averaging (section-3.3.4.2), and the fourth simulation-run (RUN454) uses the level-set method with the optimum averaging and a reduced Gibbs-Thomson coefficient. All of the simulation runs have the same initial $\mathrm{S} / \mathrm{L}$ interface configuration as shown in Figure-4.17.

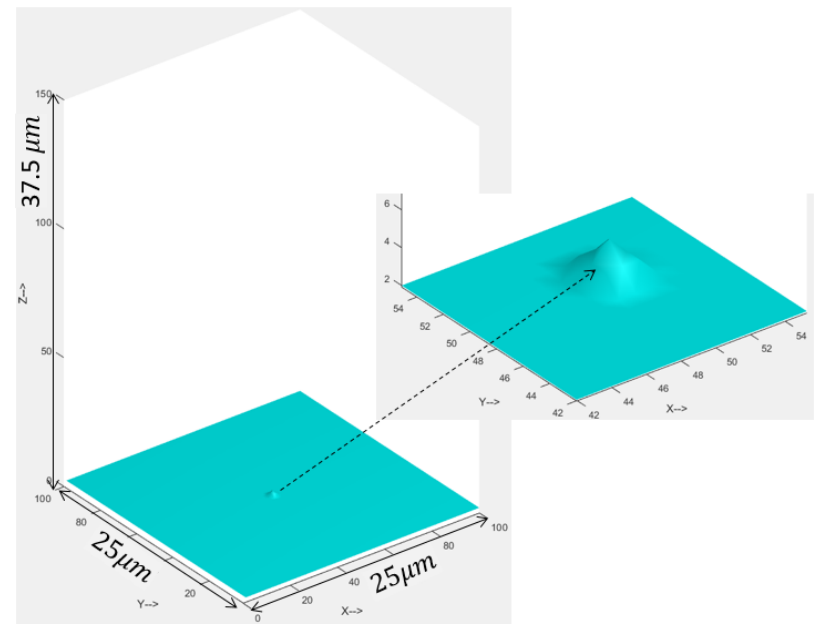

Figure-4.17: The initial S/L interface for RUN451, RUN452, RUN453, and RUN454.

The initial morphology, as shown in Figure-4.17, is a flat $\mathrm{S} / \mathrm{L}$ interface with a small nucleus in the middle with $0.75 \mu \mathrm{m}$ width and $0.5 \mu \mathrm{m}$ height. The purpose of the nucleus is to produce a convex interface that reduces the liquidus temperature and enhances the growth. If a flat interface is used without the nucleus, the initial flat interface will advance without producing a dendrite. Figure-4.18 shows the dendrite produced in RUN451, and Figure4.19 shows the dendrite produced produced in RUN452. 

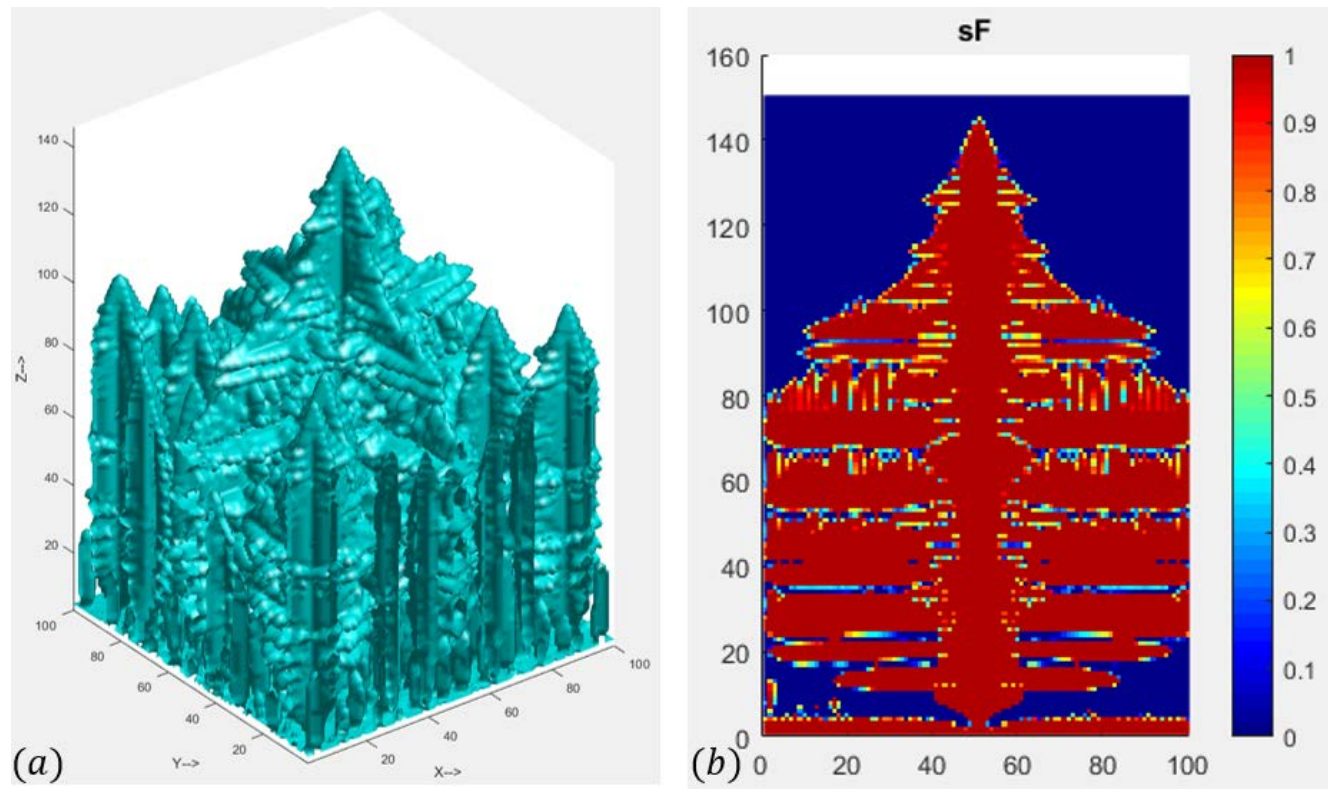

Figure-4.18: (a) the developed microstructure of RUN451 that uses cell-count method, and (b) a cross section of the solid fraction through x-axis. [RUN451]

When a cell-count method is used (Figure-4.18), a main dendrite is developed in the middle of the computational domain and some smaller dendrites are produced within the computational domain. The main dendrite is developed from the initial nucleus, and the other dendrites are grown from disturbances produced on the initially flat $\mathrm{S} / \mathrm{L}$ interface. The disturbances occur because of the cell-count method that impose some uncontrolled numerical noise in the curvature computation. This noise starts from the initial nucleus surrounding and propagates through the domain disturbing the flat $\mathrm{S} / \mathrm{L}$ interface and produces smaller perturbations. Eventually, the perturbations grow and form full columnar dendrites as shown in Figure-4.20. 

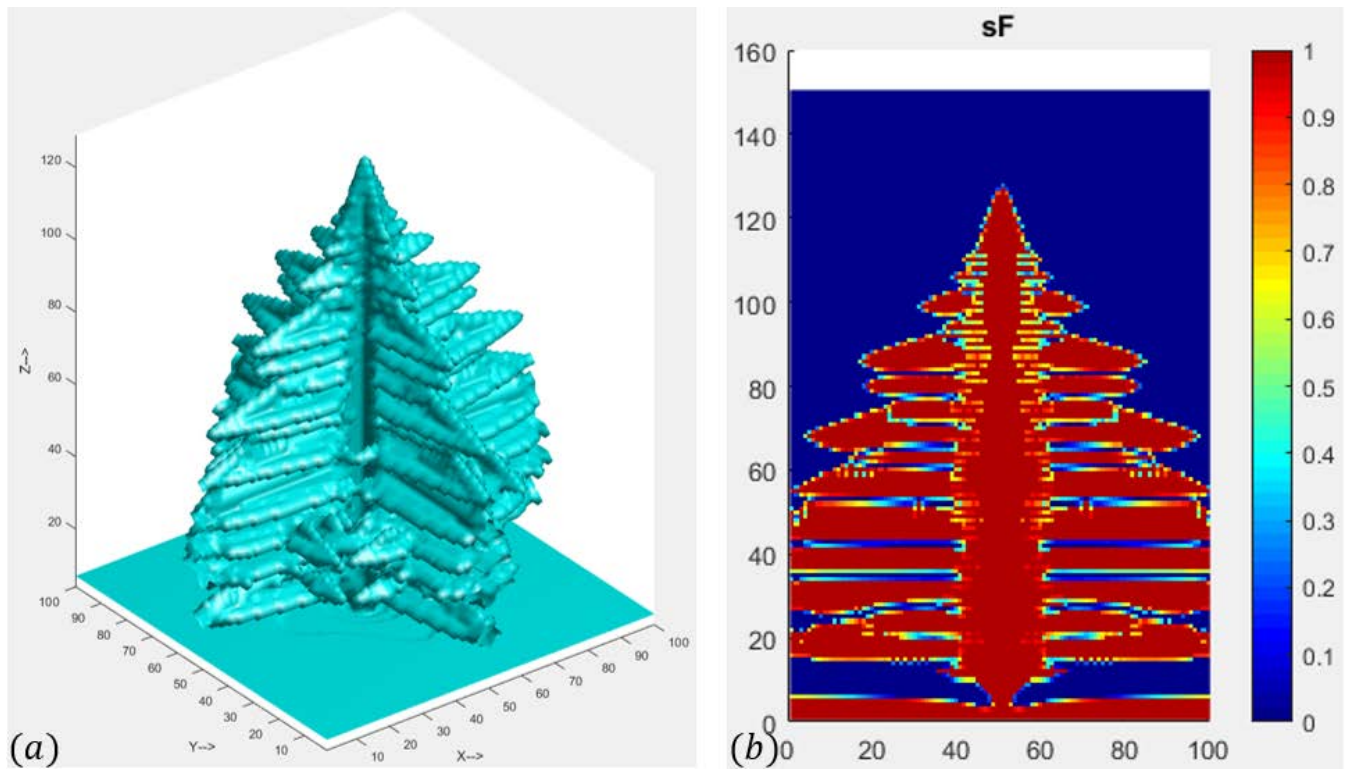

Figure-4.19: The developed microstructure of RUN452 that uses level-set method, and (b) a cross section of the solid fraction through $\mathrm{x}$-axis. [RUN452]

Figure-4.19 shows the resulting microstructure using the level-set method with averaging weights, as describe in section-3.3.4.2. In comparison to cell-count method, level-set method does not produce strong numerical noise and does not disturb the initial flat S/L interface. As a result, one dendrite is grown from the initial nucleus and develops secondary and some tertiary arms.

Figure-4.20a shows the initial disturbances of the S/L interface, and Figure-4.20c shows the initial perturbations. These perturbations act as nuclei and produce dendrites as shown in Figure-4.20d. 

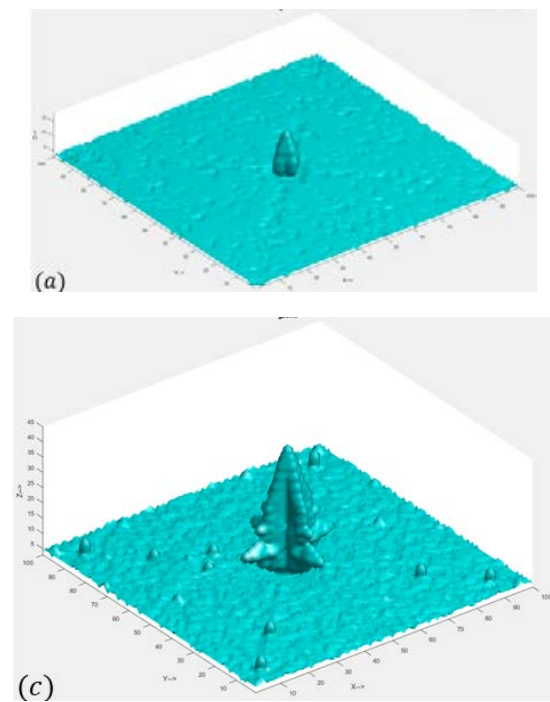
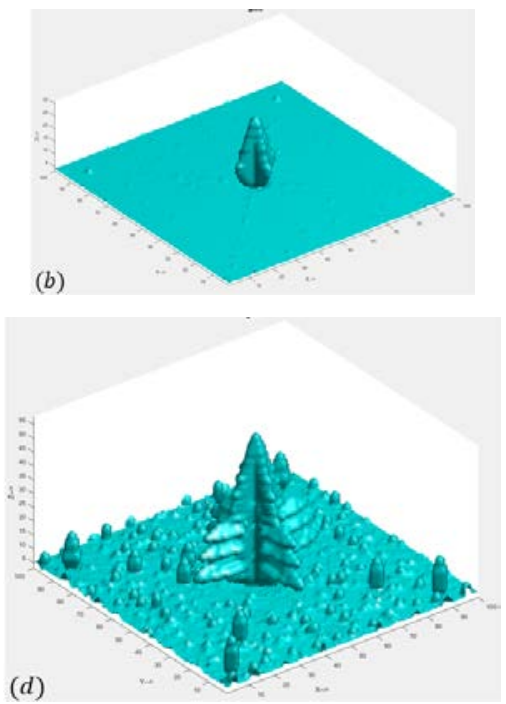

Figure-4.20: The disturbance of the initial $\mathrm{S} / \mathrm{L}$ interface due to cell-count method at (a) $0.0011 \mathrm{sec}$, (b) $0.0022 \mathrm{sec}$, (c) $0.0033 \mathrm{sec}$, and (d) $0.0045 \mathrm{sec}$.

Figure-4.21 shows the dendrite produced in RUN453, and Figure-4.22 shows the dendrite produced in RUN454.
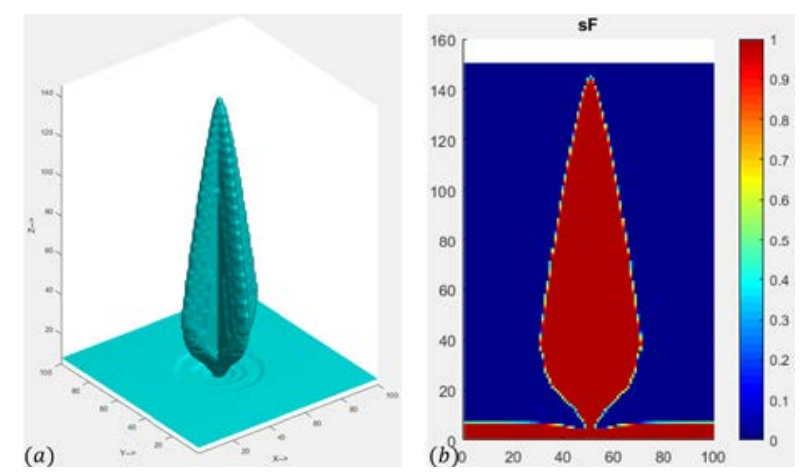

Figure-4.21: The developed microstructure of RUN453 that uses level-set method with optimum averaging, and (b) a cross section of the solid fraction through $\mathrm{x}$-axis. [RUN453]

Figure-4.21 shows the resulting dendrite when the level-set method with optimum weights averaging is used. In contrast to the dendrite shown in Figure-4.19, the developed dendrite with optimum weights averaging is smoother and less fractal. This is so because the 
curvature calculation using optimum averaging values is more accurate, as predicted by the sphere test (section-3.3.5), which reduces the numerical noise. Therefore, a simple and smooth shape is maintained for a longer time, and the structure will be fractal eventually if a larger computational domain is used.

Figure-4.22 shows the resulting dendrite of RUN454, when the level-set method with optimum weights averaging is used and the value of $\Gamma$ is reduced by half, so $\Gamma=0.5^{-7} \mathrm{~K}$. $m$. Gibbs-Thomson coefficient enhances the effect of surface energy so that a lower value of it reduces the action of the surface energy. Since the surface energy tends to flatten or smoothen the $\mathrm{S} / \mathrm{L}$ interface (as described in section-1.7.1), it is expected that a smaller value of $\Gamma$ will produce more fractal dendrite, in comparison to the case when a higher value of $\Gamma$ is used as in RUN453.
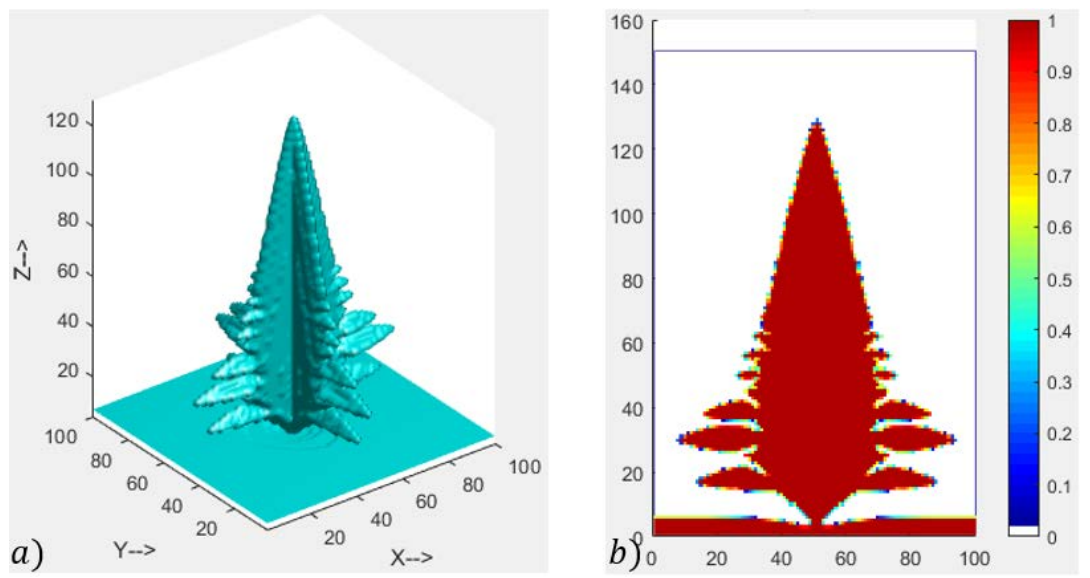

Figure-4.22: The developed microstructure of RUN454 that uses level-set method with optimum averaging and $\Gamma=0.5^{-7} \mathrm{~K} \cdot \mathrm{m}$, and (b) a cross section of the solid fraction through x-axis. [RUN454] 
The growth of the columnar, or smooth, structure is slower than the dendritic structure. For example, the simulation time for RUN452 (dendritic) was equal to $0.0098 \mathrm{sec}$ and the simulation time for RUN453 (columnar) was equal to $0.0126 \mathrm{sec}$. This is for the same reason as the case of spherical growth (section-4.1) in which smoother $\mathrm{S} / \mathrm{L}$ interface has a smaller total area for diffusion with a slower diffusion process. As a result, the solutes removal from the $\mathrm{S} / \mathrm{L}$ interface is slower and so the growth rate.

\subsection{Dendrite Growth Simulations Including Heat Diffusion Solver}

The heat diffusion is not solved for all previous simulation runs because the isothermal case is considered with a uniform temperature within the computational domain. In this section two simulation runs (RUN461 and RUN462) are investigated in which the heat diffusion is solved as described in section-3.3.5. Both runs have the same simulation parameters with nominal alloy composition $16 \mathrm{wt} \%$ of $\mathrm{Cr}$ and $0.05 \mathrm{wt} \%$ of $\mathrm{C}$, initial temperature $=1508^{\circ} \mathrm{C}$, anisotropy coefficients 0.25 , and Gibbs-Thomson coefficient $\Gamma=$ $1^{-7} K \cdot m$. Since the heat diffusion equation will be solved, more alloy properties are needed which are thermal conductivity $k_{t h}=43 / 100 \mathrm{~J} / \mathrm{K} \cdot \mathrm{m}^{3}$, latent heat of fusion $L h=17.2 \times 10^{6} \mathrm{~J} / \mathrm{m}^{3}$, heat capacity $C p=3.1 \times 10^{7} \mathrm{~J} / \mathrm{K} \cdot \mathrm{m}^{3}, \quad$ and density $\rho=$ $6800 \mathrm{~kg} / \mathrm{m}^{3}$.

Periodical thermal boundary conditions are used for all sides of the computational domain. In addition, an insulated boundary condition is assigned to the top of the domain, and a temperature gradient equal to $0.1^{\circ} \mathrm{C} / \mu \mathrm{m}$ is assigned to the bottom of the domain to add the 
cooling effect. For solute transport, periodical boundary conditions are used for side surfaces of the domain, and isolated boundaries are used for the top and the bottom of the domain.

Although both simulation-runs have the same simulation parameters, they have different initial S/L interface configurations as shown in Figure-4.23, wherein RUN461 has one initial nucleus at the middle of the computational domain and RUN462 has a rough initial $\mathrm{S} / \mathrm{L}$ interface. The computation time of these simulations is the longest because the used iteration technique to account for the thermal diffusion performs 5 to 10 interpolation calculations for each interfacial cell at every time step, as described in section-3.3.5.
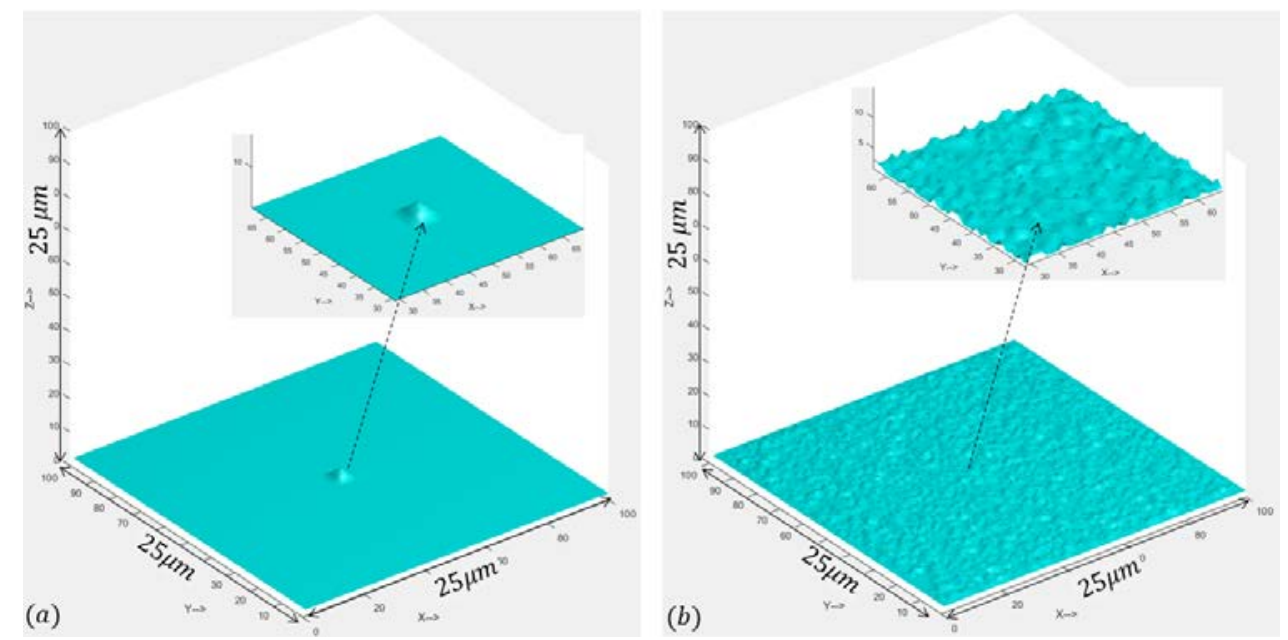

Figure-4.23: (a) the initial S/L interface of RUN461, and (b) the initial S/L interface of RUN462.

Figure-4.23a shows the initial S/L interface of RUN 461 in which one small nucleus $(0.75$ $\mu m$ width and $0.5 \mu m$ height) is located at the center of the domain to promote the growth of the dendrite. Unlikely, Figure-4.23b shows the case when the initial $\mathrm{S} / \mathrm{L}$ interface is 
microscopically rough, i.e. four thousand initial nuclei with different size are randomly distributed. The induced roughness will change the curvature of the initial S/L interface that promotes many dendrites to grow from different locations.

The next figures (Figure-4.24, 4.25, 4.26) show the growing microstructure for RUN461 at times equal to $0.0078,0.019$, and $0.027 \mathrm{sec}$ respectively.
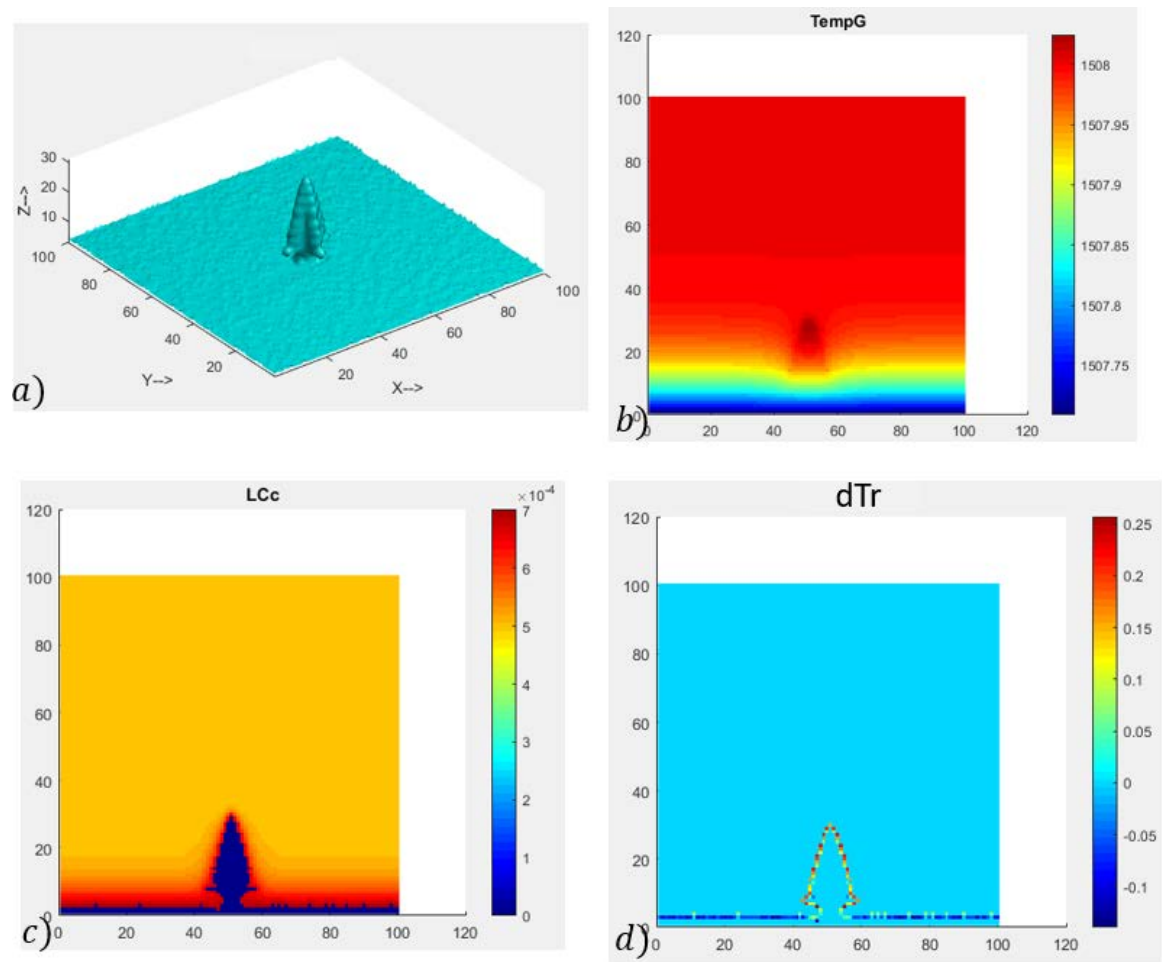

Figure-4.24: For RUN461 at $\mathrm{t}=0.0078 \mathrm{sec}$, (a) the growth dendrite, (b) the rejected heat, (c) the rejected solute (carbon), and (d) the capillarity undercooling at a cross section.

Figure-4.24a shows a growth of a dendrite from the initial nucleus at time equal to 0.0078 sec. The initial S/L interface starts to be disturbed because the cell-count method is used. Figure-4.24b shows the temperature distribution within a cross section through the 
computational domain, and it shows how the releasing of latent heat increases the temperature around the dendrite tip. The maximum and minimum temperature are equal to $1508.1^{\circ} \mathrm{C}$ and $1507.75^{\circ} \mathrm{C}$ respectively. In addition, the temperature is minimum at the bottom of the domain since a cooling boundary condition is assumed there. Figure-4.24c shows the concentration of carbon in liquid, which is formed due to the solute rejection process. Well-defined layers of solutes are formed around the growing dendrite, and the rest of the domain is at the initial solute concentration. Finally, Figure-4.24d shows the calculation of capillarity undercooling $(\mathrm{dTr})$ at a cross section of the computational domain in which the range of $\mathrm{d} \operatorname{Tr}$ is $-0.1^{\circ} \mathrm{C}$ to $0.25^{\circ} \mathrm{C}$.
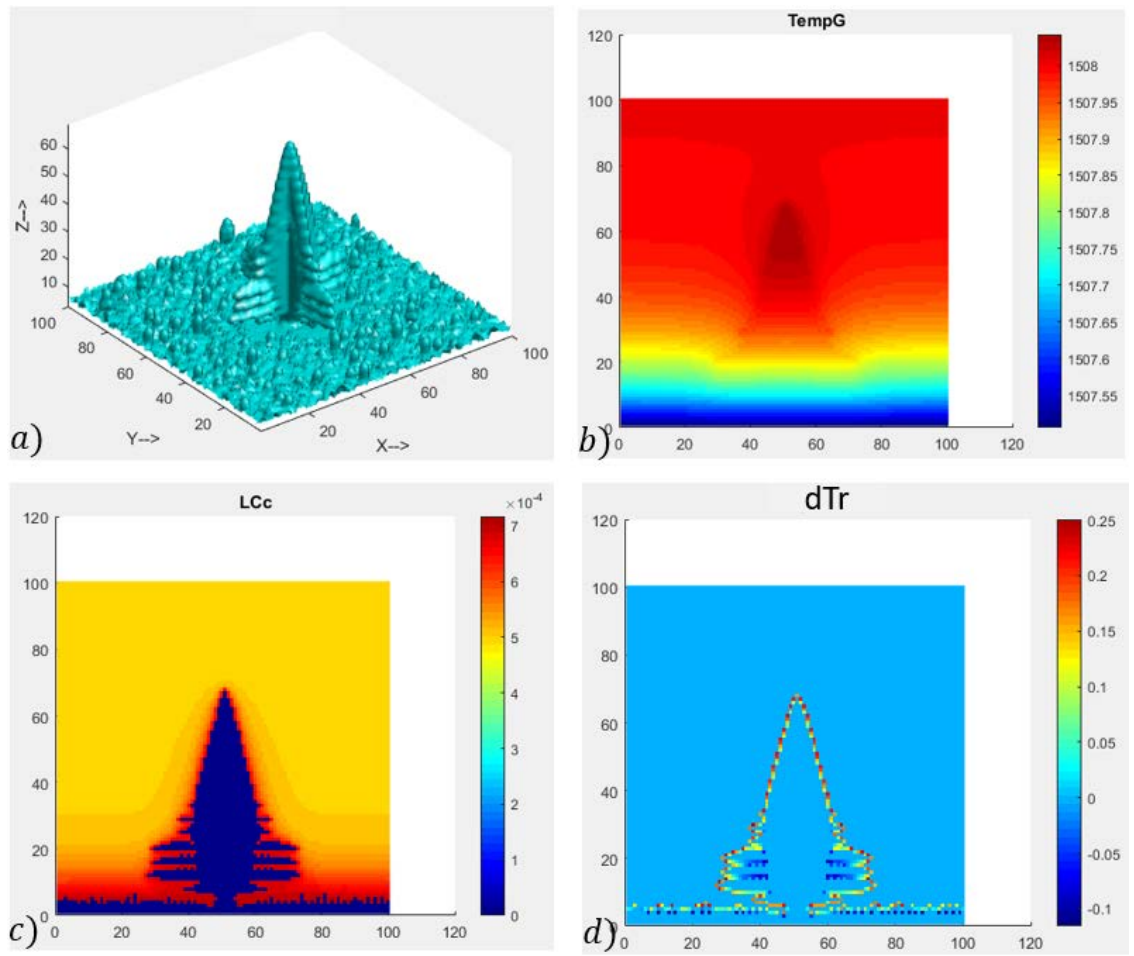

Figure-4.25: For RUN461 at $\mathrm{t}=0.019 \mathrm{sec}$, (a) the growth dendrite, (b) the rejected heat, (c) the rejected solute (carbon), and (d) the capillarity undercooling at a cross section. 
Figure-4.25 shows the growth of the dendrite at time equal to $0.019 \mathrm{sec}$. The dendrite becomes longer and grows secondary arms, and some perturbations start to form nuclei. Figure- $4.25 \mathrm{~b}$ and $\mathrm{c}$ show the temperature distribution and the carbon concentration around the growing dendrite respectively. Finally, Figure- $4.25 \mathrm{~d}$ shows that the absolute values of $\mathrm{dTr}$ are increased since the dendrite becomes more fractal.
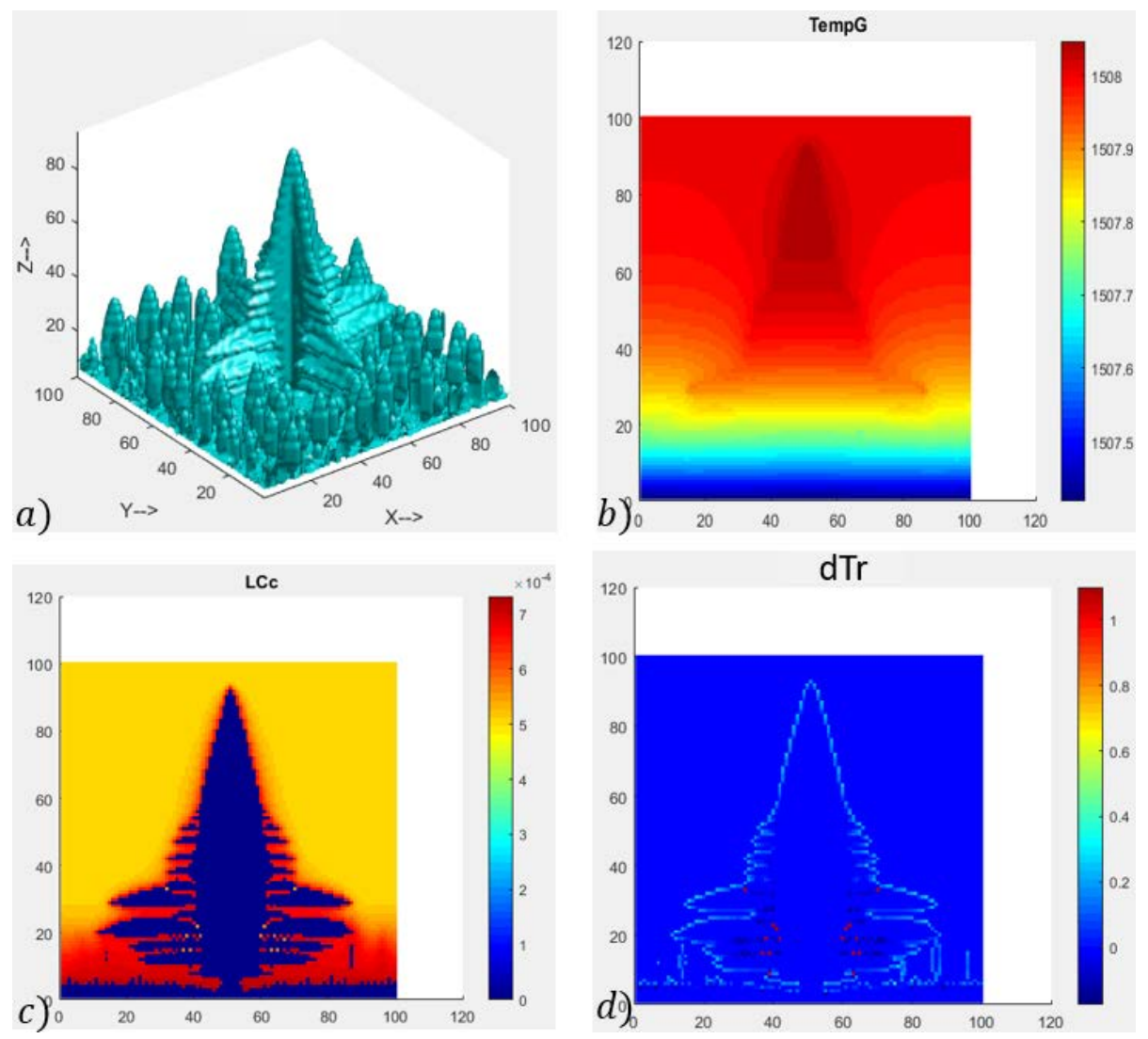

Figure-4.26: For RUN461 at $\mathrm{t}=0.027 \mathrm{sec}$, (a) the growth dendrite, (b) the rejected heat, (c) the rejected solute (carbon), and (d) the capillarity undercooling at a cross section.

Figure-4.26a shows the developed dendrite at the end of the simulation, at time equal to $0.027 \mathrm{sec}$. The dendrite is well developed with many secondary arms and a parabolic tip. In addition, some columnar dendrites grow from the formed nuclei due to the produced 
numerical noise. Figure-4.26b shows the temperature distribution and the released latent heat around the dendrite. Figure-4.26c shows the solute concentration around the dendrite which indicates that the diffusion process promotes the growth. Finally, Figure-4.26d shows that the value of $\mathrm{dTr}$ is increased up to $1^{\circ} \mathrm{C}$, because the dendrite shape becomes more complex and acquires sharp features.

Figure-4.27 shows the result of RUN462 in which the initial S/L interface is rough (see Figure-4.23b). It is expected for such initial configuration that many dendrites will start to grow and few of them will survive because of the competitive growth. Figure-4.27a shows that many columnar dendrites are developed at the end of the simulation (at $\mathrm{t}=0.03 \mathrm{sec}$ ), and Figure-4.27b shows a cross section of the solid fraction field. The width of one columnar dendrite is approximately equal to $3 \mu \mathrm{m}$, and no secondary arms are developed because there are many columnar dendrites within the domain. Figure-4.27c illustrates the temperature distribution and shows that the temperature at the solidification front is higher due to the release of the latent heat. Meanwhile, the temperature decreases as we approach the bottom of the domain due to cooling effect. In addition, the temperature distribution becomes uniform as we go far from the solidification front. Figure-4.27d illustrates the solute concentration in liquid that shows a layer with a high solute concentration is formed around each columnar. The developed concentration layers interact with each other, this is called soft impingement, affecting the microstructure evolution due to the competitive growth. 

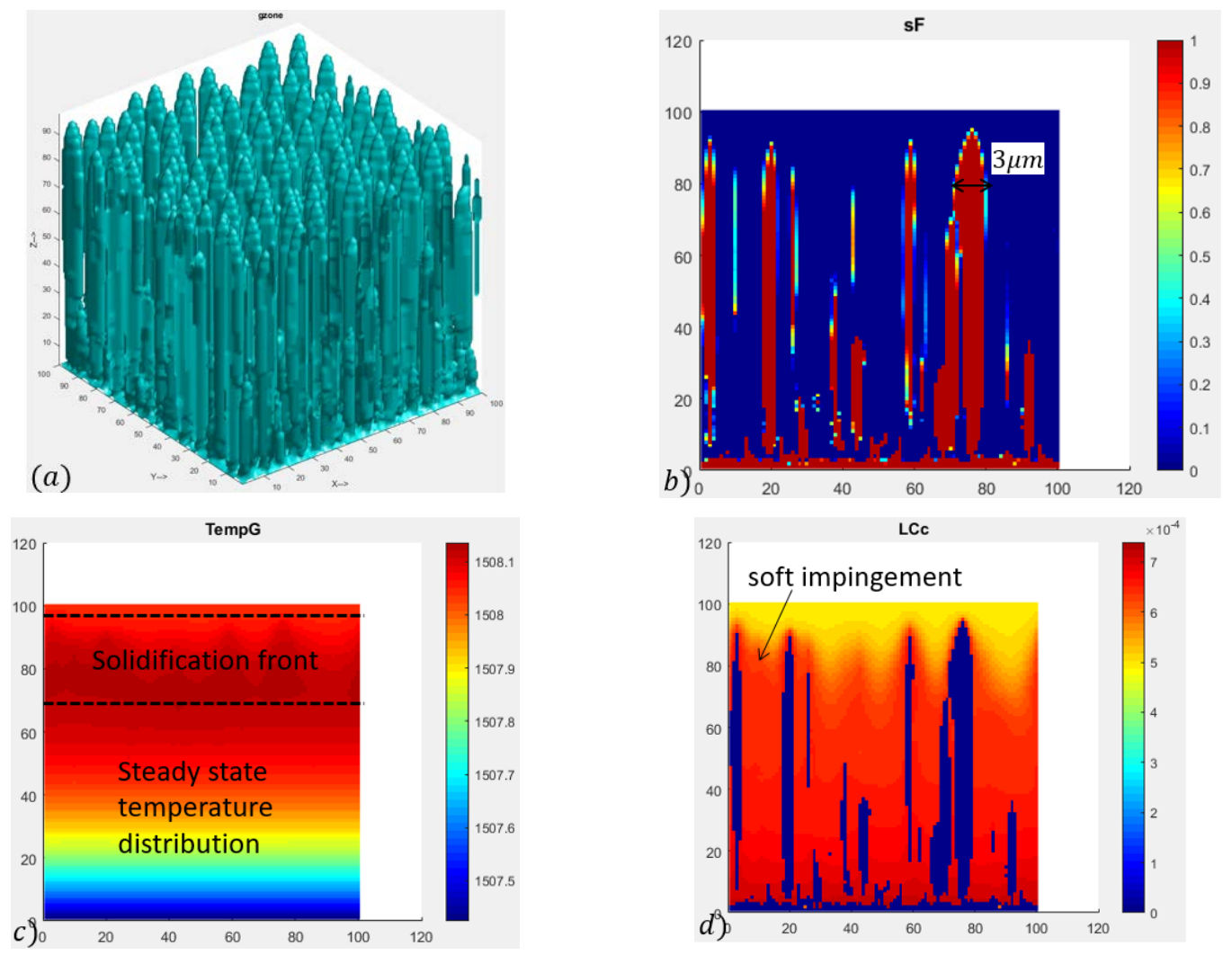

Figure-4.27: The results of RUN462 at $\mathrm{t}=0.03 \mathrm{sec}$. (a) the developed microstructure, (b) a cross section of the solid fraction field, (c) the temperature distribution, (4) the rejected solute.

Figure-4.28 shows four different stages of the microstructure evolution of RUN462. In order to study the competitive growth, a MATLAB code is written to count the number of dendrites at any time. It is found that the number of surviving dendrites decreases with time because some of them will grow at the expense of others. For example, there were 399 dendrites at $\mathrm{t}=0.0078 \mathrm{sec}, 206$ dendrites at $\mathrm{t}=0.0156 \mathrm{sec}, 161$ dendrites at $\mathrm{t}=0.0234 \mathrm{sec}$, and 68 dendrites at $\mathrm{t}=0.03 \mathrm{sec}$, as shown in Figure-4.28. By comparing the two circularwindows in Figure-4.28c and Figure-4.28d, there are fewer dendrites in Figure-4.28d. As 
a result, the primary arm spacing (section-1.7) increases in Figure-4.28d since the number of columnar dendrites decreases. It is expected that even fewer dendrites will survive and they will grow at the expense of the others after a longer simulation time.
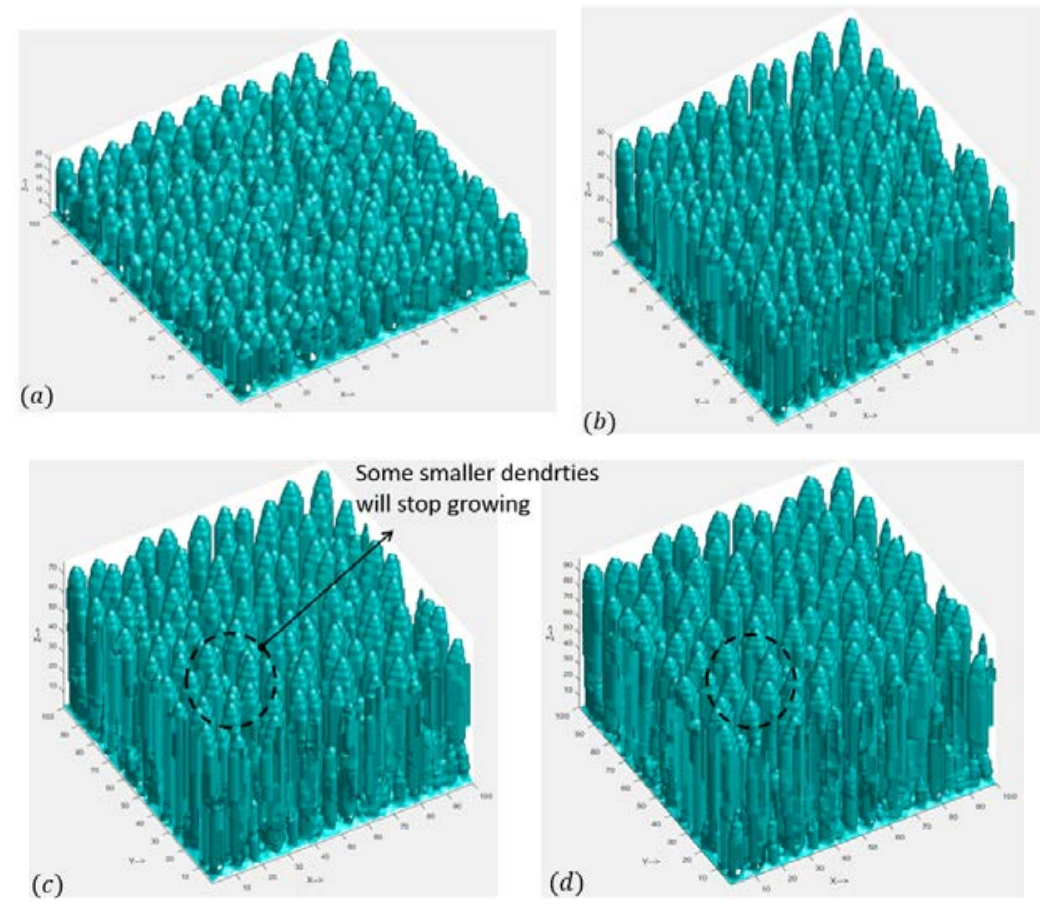

Figure-4.28: The developed microstructure at (a) $\mathrm{T}=0.0078 \mathrm{sec}$, (b) $\mathrm{T}=0.0156 \mathrm{sec}$, (c) $\mathrm{T}=0.0234 \mathrm{sec}$, and (d) $\mathrm{T}=0.0234 \mathrm{sec}$. [RUN462]

The initial configuration and state of the system are artificial one that does not represent the steady state. Therefore, the system goes through a transient state at the beginning of the simulation trying to reach the steady state. Eventually, the heat and mass distribution become steady and also the growth velocity. There is one steady state configuration for any specific solidification system with specific solidification parameters regardless to the initial $\mathrm{S} / \mathrm{L}$ interface configuration. The steady state determines the microstructure properties such as grain density and segregation. 
Figure-4.29 shows the temperature profile along a line located at the center of the computational domain of RUN462 and extended from the bottom to the top. Initially the temperature is constant and uniform $\left(1508^{\circ} \mathrm{C}\right)$, so the temperature profile is as shown by the dotted line in the figure. Figure-4.29a shows the temperature profile after 1000 time step $(0.0039 \mathrm{sec})$ which is changed due to the heat diffusion. The temperature at the bottom (point-A) is less than the initial temperature (approximately equal to $1507.8^{\circ} \mathrm{C}$ ) since the heat is extracted from the bottom. On the other hand, the temperature at the top of the domain (point-B) is yet not changed. The difference in temperature from the bottom and the top of the domain derives the heat diffusion process.
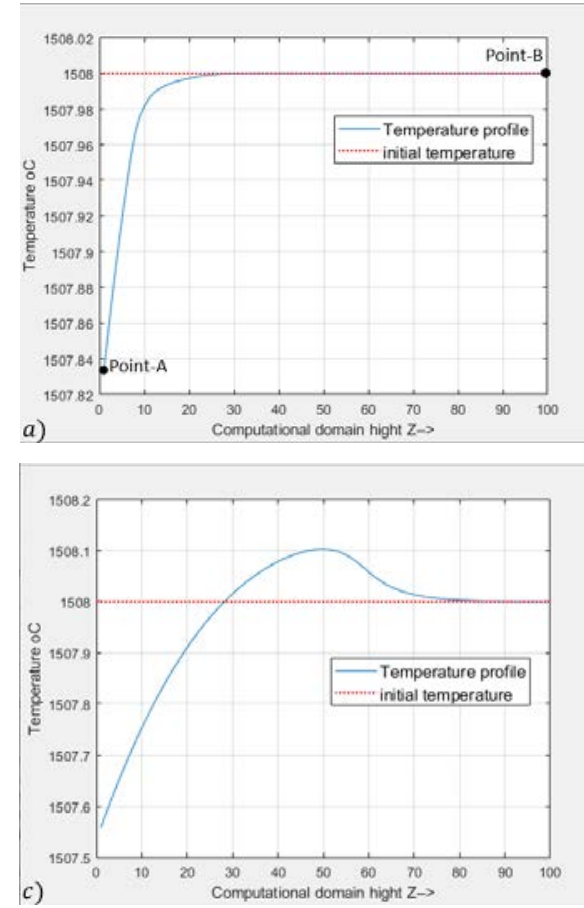
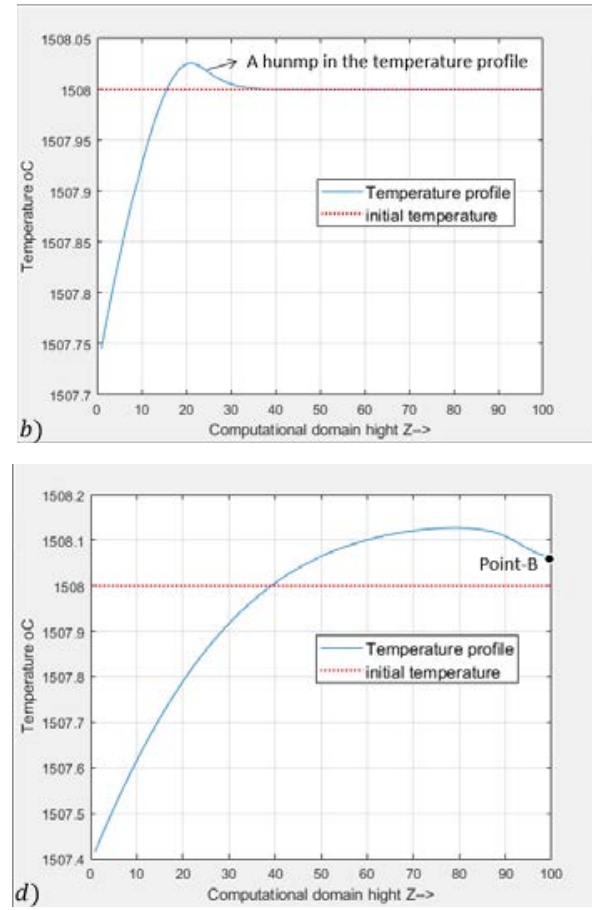

Figure-4.29: The temperature profile at (a) 1000 time step $(0.0039 \mathrm{sec})$, (b) 2000 time step $(0.0078 \mathrm{sec})$, (c) 5000 time step $(0.019 \mathrm{sec})$, and (d) 8000 time step $(0.031 \mathrm{sec})$. 
Figure- $4.29 \mathrm{~b}$ shows the temperature profile at time step $2000(0.0078 \mathrm{sec})$ on which the heat is accumulated forming a hump in the temperature profile. This temperature hump is due to the releasing of the latent heat at the solidification front, and the temperature decreases until it becomes equal to the initial temperature. At the time step 2000, the maximum temperature of the temperature hump is equal to $1508.01{ }^{\circ} \mathrm{C}$, which slightly above the initial temperature. As the solidification process continue more heat is released and so more heat is accumulated, and the temperature hump becomes higher and wider, as shown in Figure-4.29c. At this stage (time step 5000 and $0.019 \mathrm{sec}$ ), the maximum temperature is equal to $1508.1^{\circ} \mathrm{C}$. Figure-4.29d shows the temperature profile at the end of simulation in which the solidification front reaches the top of the control volume. The temperature profile becomes smoother and some interaction with the boundary condition at the top of the domain occurs (point-B). The result shows that the releasing of latent heat increases the maximum temperature at the solidification front by $0.12{ }^{\circ} \mathrm{C}$. It is expected that this value will decrease if the used thermal conductivity is not reduced. Therefore, the effect of latent heat releasing is not significant for this simulation, and a simple temperature profile can be imposed on the computational domain without solving the heat diffusion equation. 


\section{Chapter-5: Predicting the Microstructure of the Fusion Zone}

This chapter describes how the current CA-CALPHAD model can be used to predict the microstructure of the fusion zone of weld joints. The macro/micro coupling of a temperature field and CA-CALPHAD model for a Bead-On-Plate weld joint and the effect of welding parameters on the solidification microstructure are investigated. The temperature history of the weld joint is obtained using VrWeld® [69], and specific points on the weld pool boundary are picked for which the CA-CALPHAD solidification model is run. VrWeld $₫$ can analyze a given weld joint design and calculate the macroscopic thermal field and thermal stress [70, 71], fatigue life [72], the macroscopic microstructure for low alloy steels [73], the solid-state microstructure phase transition based on the phasefield modeling [4], and the microstructure of the fusion zone based on the phase diagram linearization [3].

In this study, three types of steel alloys are investigated for the same weld joint geometry and welding procedure: 430 ferritic stainless steel, 410 martensitic stainless steel, and a duplex steel with $\mathrm{Cr} 8 \% \mathrm{wt}-\mathrm{C} 0.5 \%$ wt. Stainless steel alloys are chosen because the available CA solidification models that are based on the linearization approach cannot study them. In addition, stainless steels are practical alloys which are being used in many industrial applications. Stainless steels have high strength and corrosion resistance at high temperatures, so they are suitable for marine and energy applications. Generally, the 
stainless steels are readily weldable [74], but the weldability decreases as the carbon content increases.

One main problem with welding stainless steels is the weld decay or the weld sensitization. It is an intergranular corrosion in which chromium carbides form around the grain boundaries depleting grain boundaries of chromium. Since chromium carbides can form at a specific chromium and carbon concentration, estimating the ratio of carbon concentration to chromium concentration $(\mathrm{C} / \mathrm{Cr})$ can characterize the tendency to carbide formation and chromium depletion.

\subsection{Coupling Macroscopic Welding Case to Microscopic Solidification Model}

Figure-5.1 shows a Bead-On-Plate weld joint, as giving by [75]. The welding parameters are the welding volt is $11.2 \mathrm{~V}$, the welding current is $160 \mathrm{~A}$, and the welding speed is 140 $\mathrm{mm} / \mathrm{min}$. The material properties of $316 \mathrm{~L}$ stainless steel are used for the macroscopic thermal analysis. The double ellipsoid model [76] is used for the weld pool (Figure-5.1b), and the weld joint design is shown schematically in Figure-5.1a. Using VrWeld ${ }$, the cooling curve (T-t) at any point in the weld joint can be obtained from the FE analysis.

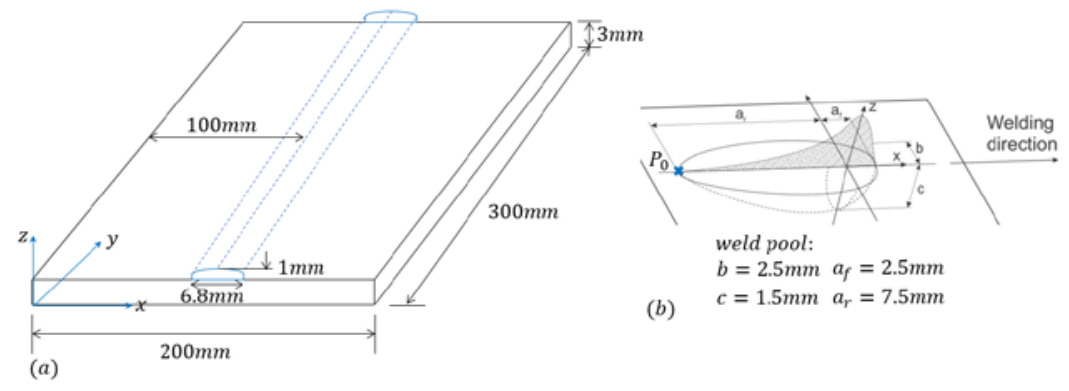

Figure-5.1: The used welding joint for macroscopic temperature calculation. 
Figure-5.2, for example, shows the cooling curve at point $\mathrm{P}_{0}$ (shown in Figure-5.1b), and the cooling rate at this point at the onset of solidification can be obtained as a corresponding $\mathrm{dT} / \mathrm{dt}$ value. However, to simplify the calculation, a secant slope is used to calculate $\mathrm{dT} / \mathrm{dt}$. For example, on the cooling curve of $\mathrm{P}_{0}$, a point at the melting temperature $(\mathrm{Tm})$ and a point with a temperature value slightly above it (T2) are used to calculate the slope or the cooling rate, as shown in Figure-5.2b.

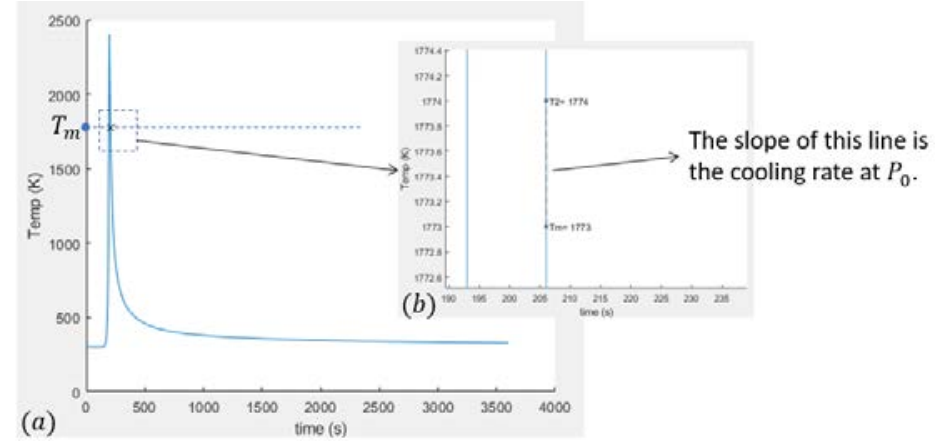

Figure-5.2: (a) the cooling curve at $\mathrm{P}_{0}$, (b) the cooling rate at $\mathrm{P}_{0}$.

The cooling rate of any point on the weld pool boundary can be calculated using the same procedure.

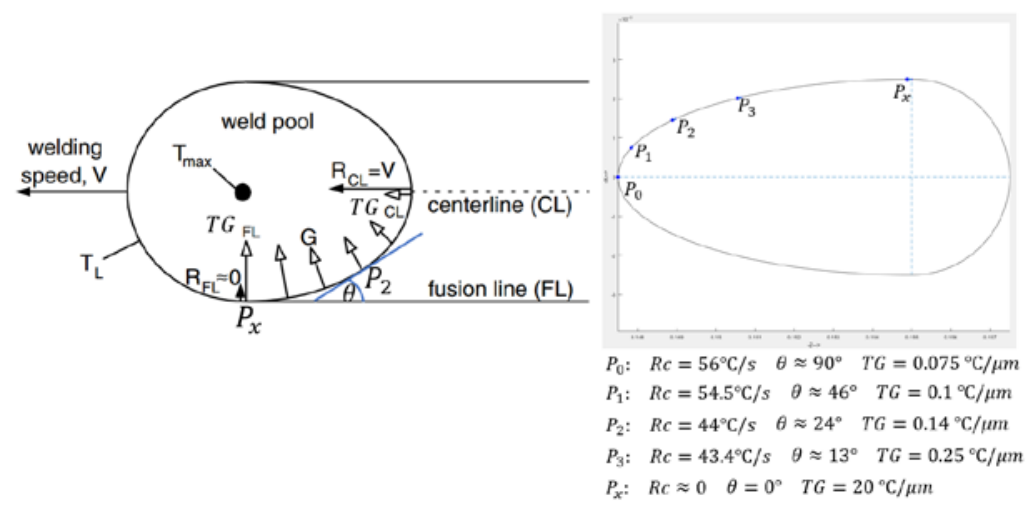

Figure-5.3: The variations of cooling rate and temperature gradient around the weld pool. [9], page-201. 
Figure-5.3a shows the variations of growth velocity and temperature gradient around the weld pool. Figure-5.3b lists the cooling rates (Rc) and the temperature gradients (TG) at some specific points on the weld pool boundary. The maximum growth velocity is at point $\mathrm{P}_{0}$ and the minimum growth velocity is at $P_{x}$. Reversely, the minimum temperature gradient is at $\mathrm{P}_{0}$ and the maximum is at $P_{x}$, as described in section-1.5.2. Once the cooling rate at any point is obtained, the temperature gradient at the point can be calculated as following:

$$
\stackrel{\overbrace{d T}^{\text {temperature gradient }}}{\frac{\overbrace{d t}}{\text { cooling rate }}}=\frac{\overbrace{d T / d t}}{d x / d t} \quad \frac{d x}{d t}=V_{\text {weld }} \cdot \sin \theta \quad \text { Eq5.1 }
$$

where, $V_{\text {weld }}$ is the velocity of welding and $\theta$ is the angle between the fusion line and the weld pool boundary at a point on the weld pool perimeter and the fusion line as shown in Figure-5.3a. By writing a MATLAB code to calculate Equation-5.1, the cooling rate (Rc) and temperature gradient (TG) for some points are calculated as listed in Figure-5.3b. The initial configuration for all simulation-runs in this chapter is the same as shown in Figure4.17.

\subsection{The Microstructure Evolution of the Weld Pool Boundary}

Referring to Figure-5.3b, the microstructure development at point $\mathrm{P}_{0}$ is studied in RUN511, and the microstructure development at point $\mathrm{P}_{2}$ is studied in RUN512. Both runs use the same simulation parameters and the cell-count method. The used simulation parameters are nominal alloy composition $\operatorname{Cr} 16 \% w t-C 0.08 \% w t$, initial temperature $=1504{ }^{\circ} \mathrm{C}$, anisotropy coefficients $=0.2$, and Gibbs-Thomson coefficient $=1^{-7} K \cdot m$. In addition, a 
linear temperature profile is assumed within the computational domain in which the temperature at the bottom is equal to the initial temperature and increases with the corresponding temperature gradient and cooling rate through the computational domain. This approach is described in section-3.3.2 and investigated in section-4.6. The microstructure of RUN511 is shown in Figure-5.4a. The microstructure is dendritic with a main large dendrite in the middle of the computational domain and secondary arms with some tertiary ones. In addition, smaller dendrites are developed within the domain because the cell-count method is used, as discussed in section-4.5. The minimum temperature in the domain is equal to $1503.707{ }^{\circ} \mathrm{C}$ which is slightly below the initial temperature, 1504 ${ }^{\circ} \mathrm{C}$, due to the cooling rate.
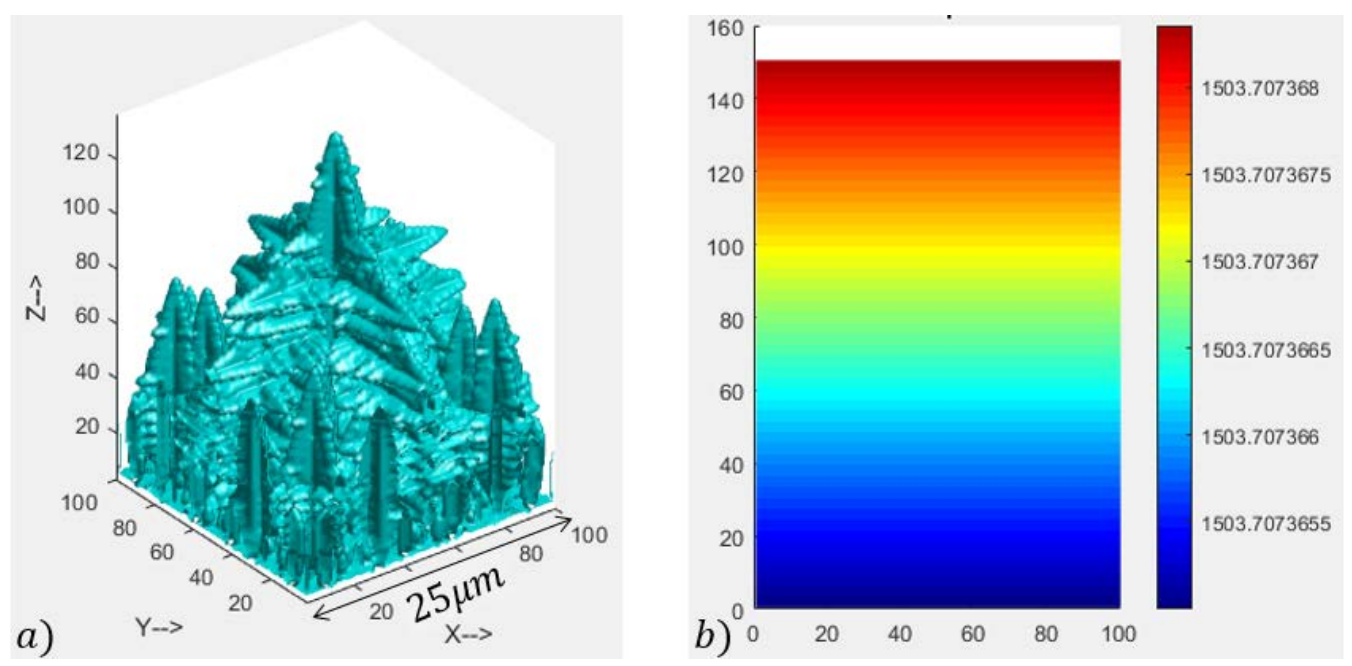

Figure-5.4: (a) the developed microstructure of $\mathrm{P}_{0}$ (RUN511) at $\mathrm{t}=0.01 \mathrm{sec}$, and (b) the resulting temperature gradient in ${ }^{\circ} \mathrm{C}$. [RUN511] 

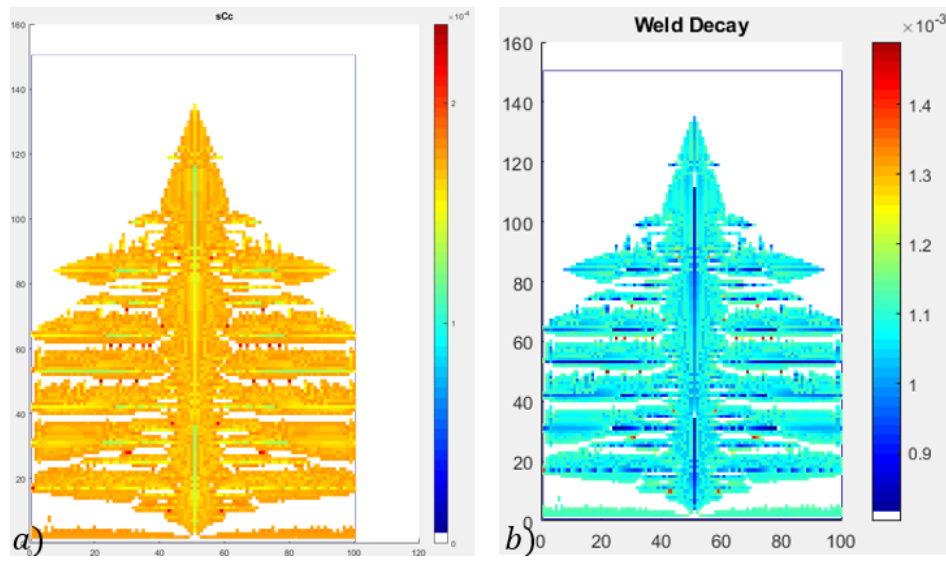

Figure-5.5: (a) the distribution of carbon in solid, and (b) the $\mathrm{C} / \mathrm{Cr}$ ratio. [RUN511]

Figure-5.5 shows the segregation of carbon in the developed dendrite and the $\mathrm{C} / \mathrm{Cr}$ ratio of RUN511. More carbon segregation is at the dendrite boundary, especially at the secondary arms. The segregation indices for RUN511 are SIndex_C $=0.6235$, SIndex_Cr $=$ 0.0034 , and $(C / C c)_{\max }=0.0015$. It can be shown from Figure-5.5b that the grain boundary of the secondary arms has higher $\mathrm{C} / \mathrm{Cr}$ ratios, which indicates they are more prone to weld decay.
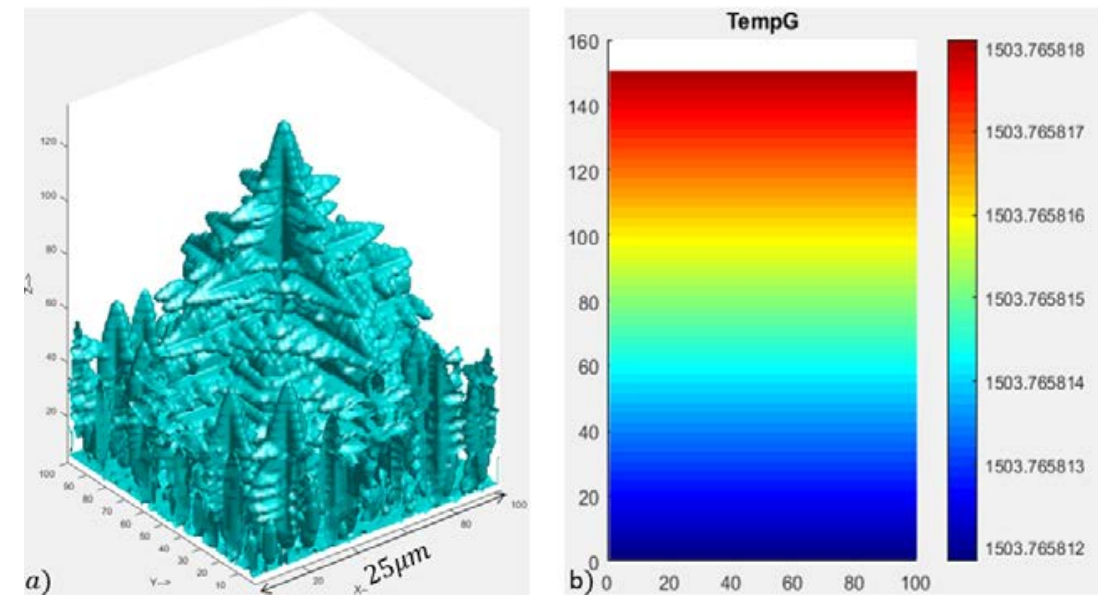

Figure-5.6: (a) the microstructure of $\mathrm{P}_{2}(\mathrm{RUN512})$ at $\mathrm{t}=0.01 \mathrm{sec}$, and (b) the resulting temperature gradient in ${ }^{\circ} \mathrm{C}$. [RUN512] 
Figure-5.6 shows the microstructure of the point $\mathrm{P}_{2}$ (RUN512) at $\mathrm{t}=0.01 \mathrm{sec}$ and the resulting temperature gradient. The microstructure is dendritic with a main large dendrite with secondary and tertiary arms. The other dendrites are due to the uncontrolled numerical noise of cell-count method. The minimum temperature is at the bottom of the control volume and equal to $1503.765^{\circ} \mathrm{C}$. Figure-5.7 shows the segregation of carbon in the dendrite and the $\mathrm{C} / \mathrm{Cr}$ ratio at $\mathrm{t}=0.01 \mathrm{sec}$ of RUN512. The carbon segregates more at the secondary arms boundary which causes higher values of $\mathrm{C} / \mathrm{Cr}$ ratios. The segregation indices for RUN512 are SIndex_C $=0.612$, SIndex_Cr $=0.0034$, and $(C / C c)_{\max }=$ 0.0015. The segregation index of carbon of RUN511 is larger than RUN512 because the cooling rate of RUN511 is higher, but the maximum $\mathrm{C} / \mathrm{Cr}$ ratios are almost equal.
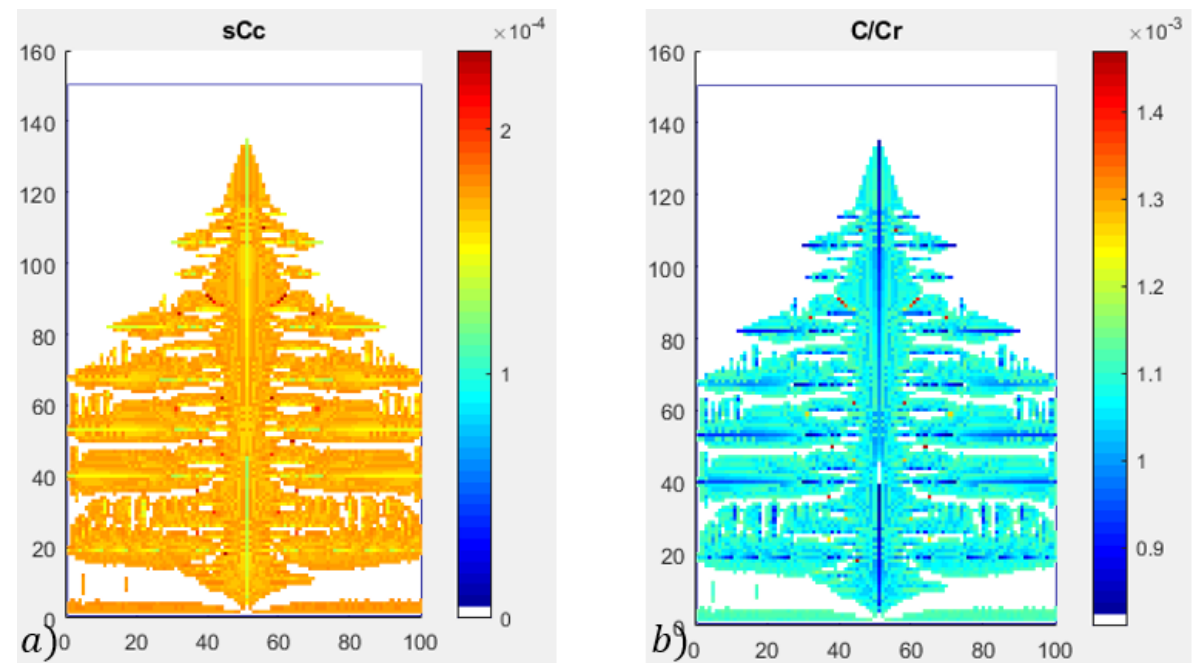

Figure-5.7: (a) the distribution of carbon in solid, and (b) the $\mathrm{C} / \mathrm{Cr}$ ratio. [RUN512].

Two more simulation-runs are investigated for the point $\mathrm{P}_{0}$ as shown in Figure-5.3b: RUN513 and RUN514. Both runs use the same simulation parameters as RUN511 and RUN512, but RUN513 uses the level-set with averaging, and RUN514 uses the level-set 
with the optimum averaging for curvature calculations, Equation-3.2.2. It is expected that one dendrite will be grown from the initial nucleus without any smaller dendrites as in RUN5111 and RUN512, because the level-set method does not disturb the initial flat S/L interface, as described in section-4.5. In addition, using the level-set method with the optimum averaging as in RUN514 will produce columnar and smooth dendrite, because the optimum averaging is more accurate and less sensitive to the numerical noise.
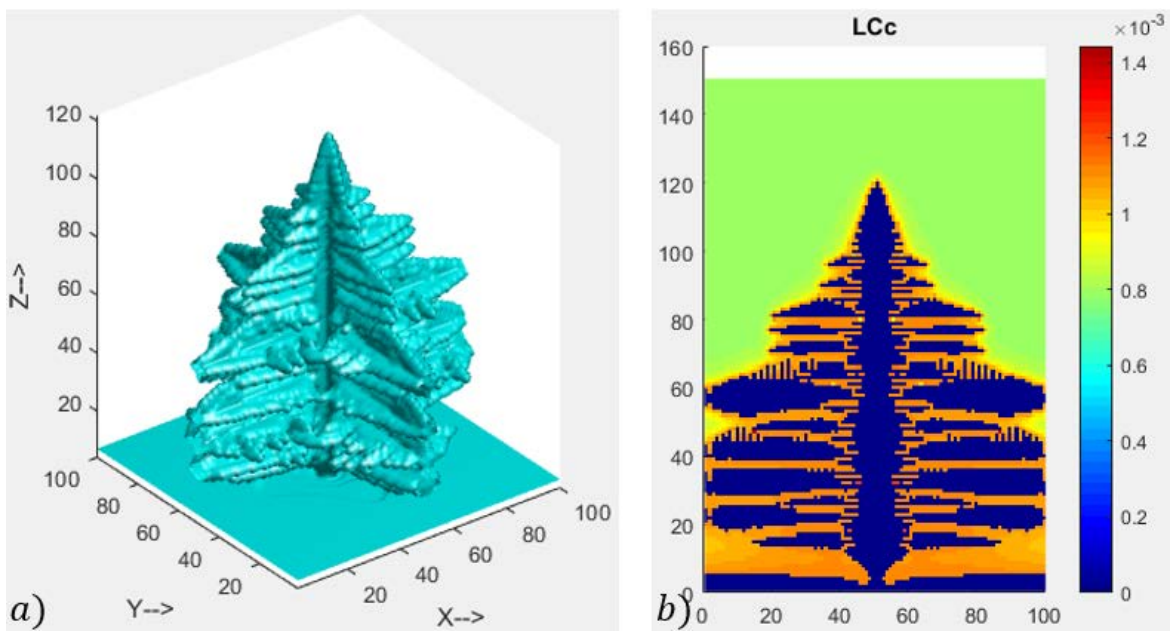

Figure-5.8: (a) the microstructure of RUN513 at $\mathrm{t}=0.011 \mathrm{sec}$, and (b) the carbon distribution in liquid. [RUN513]

Figure-5.8a shows just one dendrite in the domain with secondary and tertiary arms. This is expected since the level-set method was used. The extensive lateral growth of the dendrite arms at the bottom of the domain is due to the temperature gradient, because the bottom is colder than the top. Figure-5.8b shows the concentration of carbon in liquid in which a thin layer of solute is formed around the developed dendrite. In addition, the carbon concertation in liquid is higher at the bottom of the domain because temperature is lower, and the dendrite arms are larger at the bottom that rejects and traps more solute atoms. 
Figure-5.9 shows the carbon distribution in liquid and the $\mathrm{C} / \mathrm{Cr}$ ratios of RUN513. Carbon segregates at the dendrite boundary, and the core of the dendrite primary arm and secondary arms have less carbon concentration. The distribution of $\mathrm{C} / \mathrm{Cr}$ ratios follow the same pattern, so the boundary of the dendrite is more prone to weld decay. The maximum $\mathrm{C} / \mathrm{Cr}$ ratio is equal to 0.0059 which is four times bigger than the predicted value by using the cell-count method in RUN512. This result shows that the method of curvature computation strongly affects the microstructure composition.
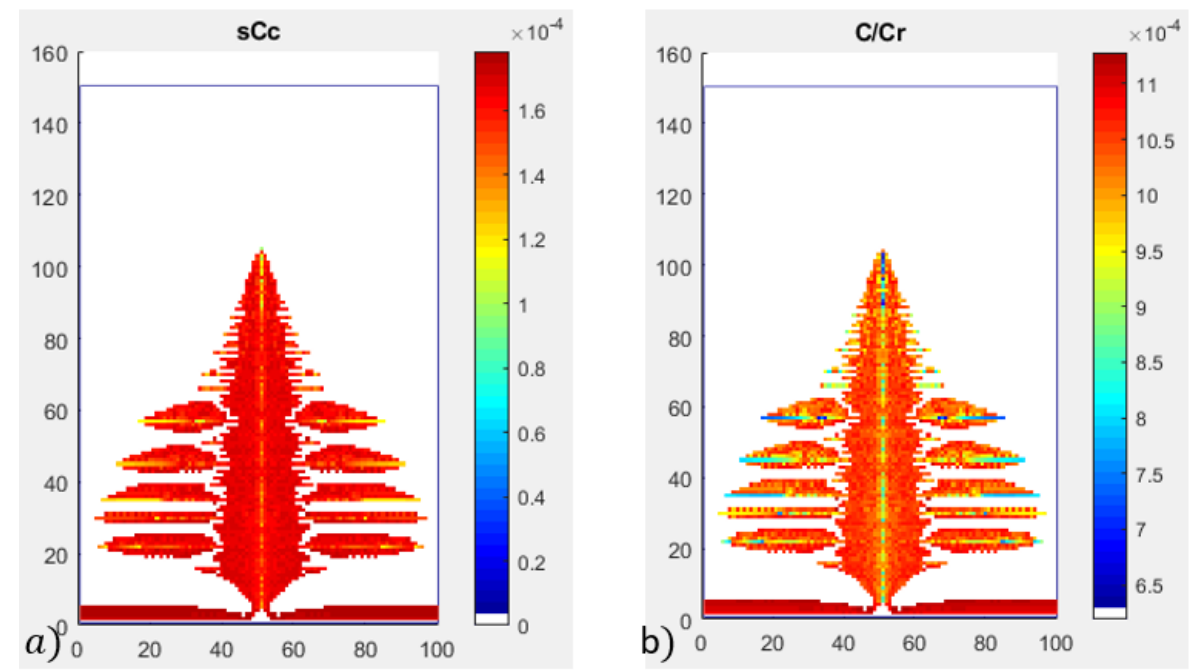

Figure-5.9: (a) the distribution of carbon in solid, and (b) the $\mathrm{C} / \mathrm{Cr}$ ratio. [RUN513].

Figure-5.10a shows the dendrite of the point P0 when the optimum averaging of the levelset is used. The dendrite is smooth, as expected, because of using the optimum averaging with level-set method. The carbon distribution in liquid (Figure-5.10b) consists of many layers surrounding the developed solid phase. Figure-5.11 shows the segregation of carbon and the distribution of C/Cr ratio of RUN514. 

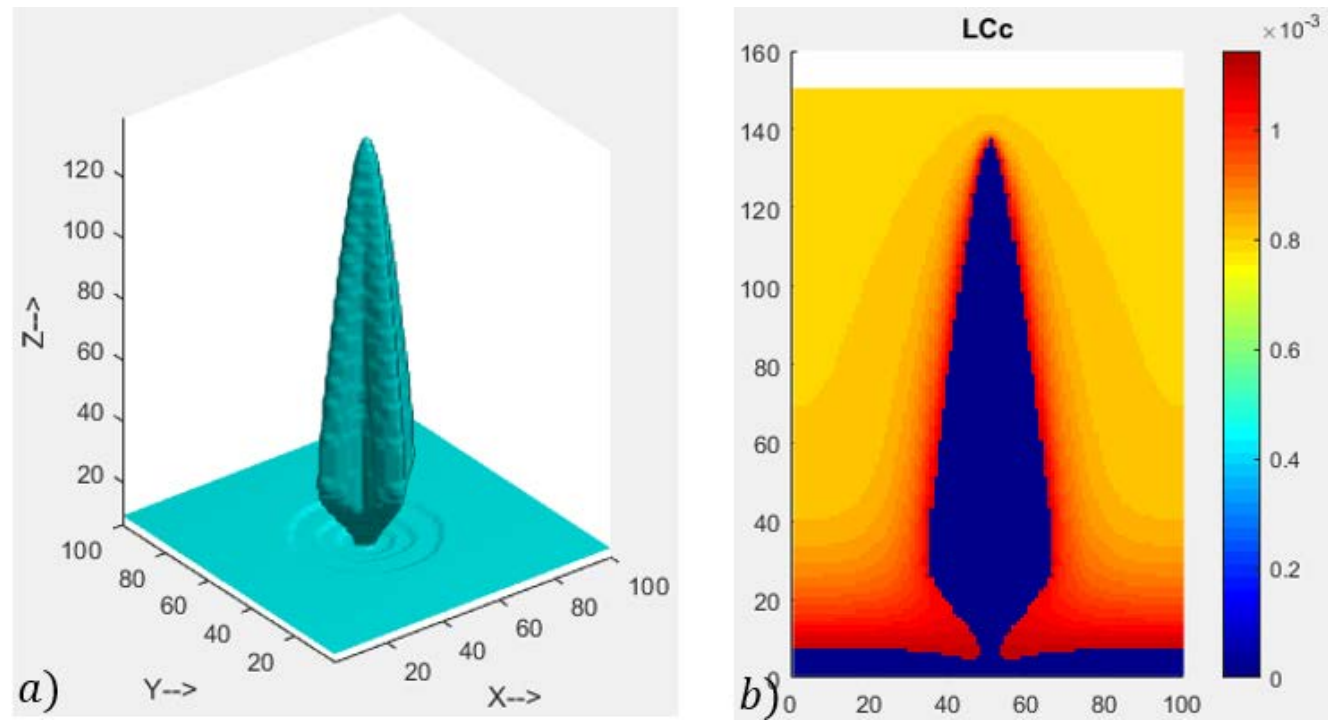

Figure-5.10: (a) the microstructure of RUN514 at $\mathrm{t}=0.012 \mathrm{sec}$, and (b) the carbon distribution in liquid. [RUN514]
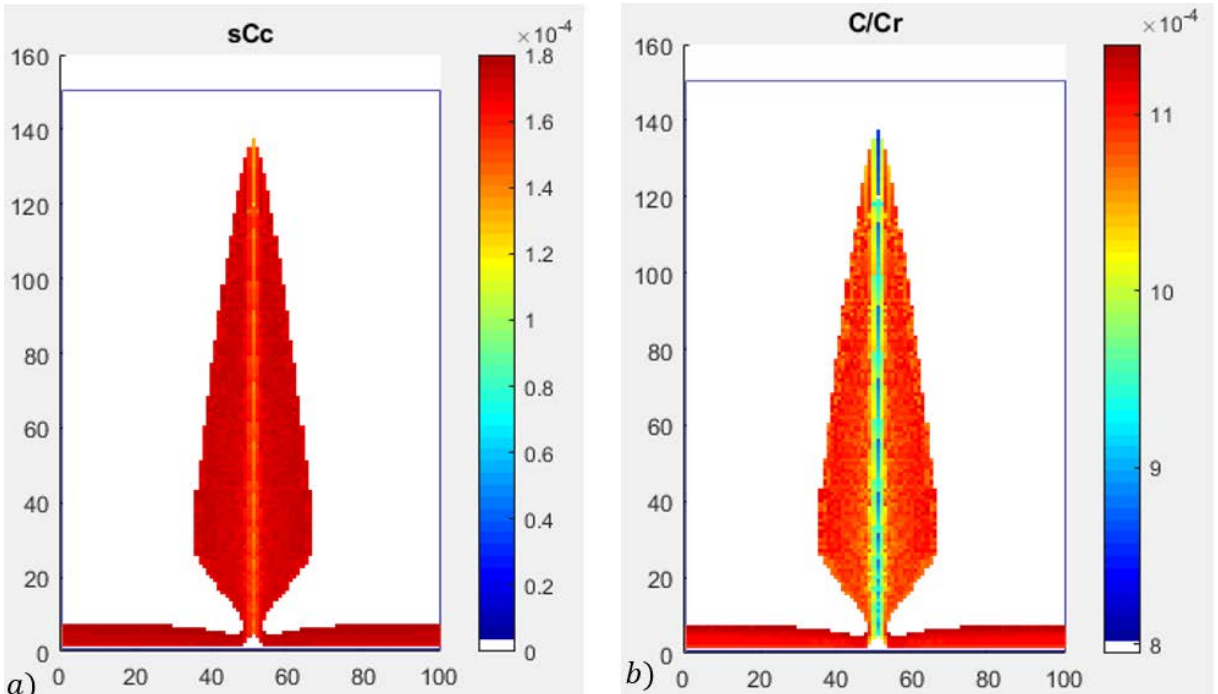

Figure-5.11: (a) the distribution of carbon in solid, and (b) the $\mathrm{C} / \mathrm{Cr}$ ratio. [RUN514]

Figure-5.11a predicts a low carbon concentration in the core of the dendrite and more segregation at the boundary. Accordingly, the boundary of the dendrite is more prone to 
weld decay. The segregation indices for RUN514 are SIndex_C $=0.32$, SIndex_Cr $=$ 0.0019 , and $(C / C c)_{\max }=0.0011$. The segregation index of carbon of RUN514 is less than RUN513 and RUN512 because the growth rate of RUN514 is the slowest.

In summary, the simulation-runs in this section show two key points. First, the microstructure around the weld pool boundary varies because of the different thermal conditions, as shown in Figure-5.3. Second, the curvature calculation method is important for the microstructure prediction accuracy. The dendrite morphology, the segregation pattern, and the growth rate are affected significantly by the used method. Therefore, improving the accuracy of the curvature calculation method is important to improve the overall prediction accuracy of the model.

\subsection{The Microstructure Evolution with a Duplex Structure}

To investigate the development of a duplex microstructure, the point $\mathrm{P}_{0}$ (as shown in Figure-5.2b) is investigated in RUN521. The used simulation parameters are nominal alloy composition $C r 8 \% w t-C 0.5 \% w t$, initial temperature $=1469.5^{\circ} \mathrm{C}$, cooling rate $=56^{\circ} \mathrm{C} / \mathrm{s}$, anisotropy coefficients $=0.2$, and Gibb-Thomson coefficient $1^{-7} K \cdot m$. In addition, a linear temperature profile is assumed within the computational domain in which the temperature at the bottom is equal to the initial temperature and increases with the corresponding temperature gradient $\left(\mathrm{TG}=0.075^{\circ} \mathrm{C} / \mu \mathrm{m}\right)$ through the computational domain. According to the thermodynamic database this alloy solidifies with ferritic microstructure 
at $1469.5^{\circ} \mathrm{C}$, but it is expecting to develop some austenitic phase at temperature equal to 1469.1 ${ }^{\circ} \mathrm{C}$, as shown in Figure-4.13.
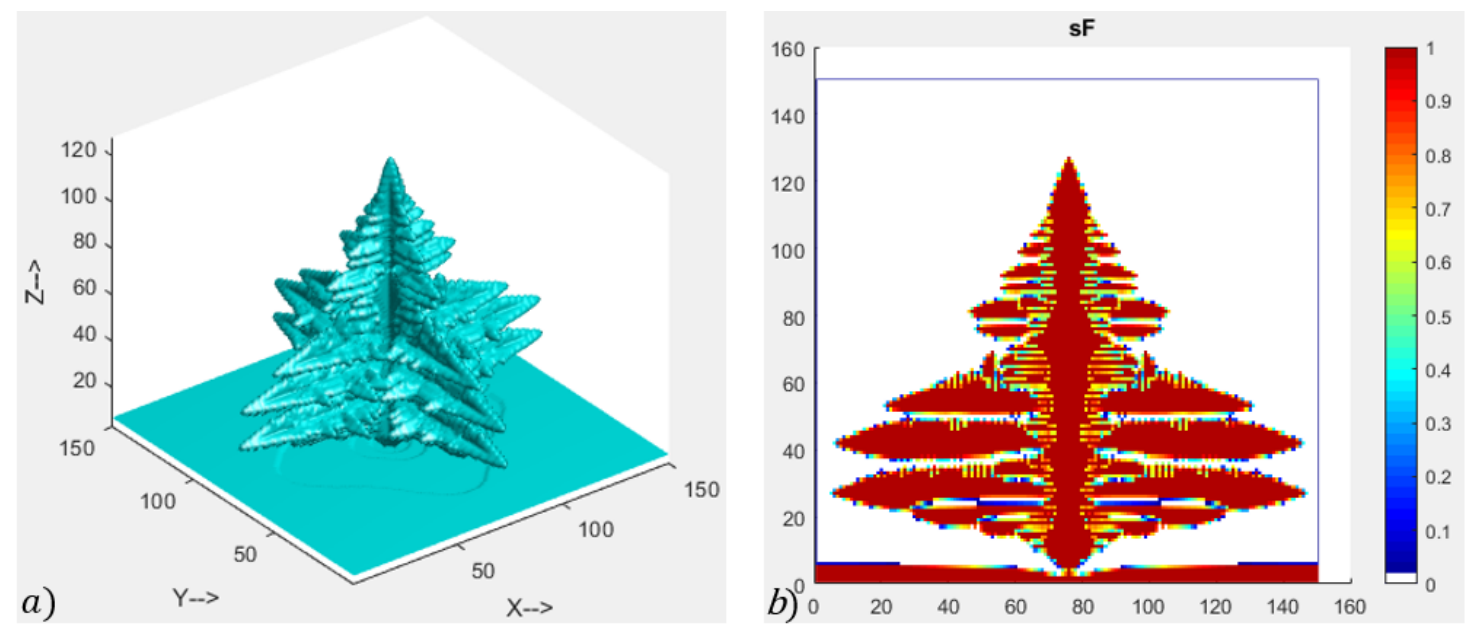

Figure-5.12: (a) the microstructure of RUN521, and (b) a cross section through the xaxis. [RUN521]

Figure-5.12a shows the microstructure of RUN521 and a cross section through the x-axis. The dendrite composes of just one dendrite on a flat $\mathrm{S} / \mathrm{L}$ interface with secondary and tertiary arms, since the level-set method was used. All cells inside the dendrite in Figure$5.12 \mathrm{~b}$ have fraction of solids equal to one, and the cells at the boundary of the dendrite have solid fractions less than one because they are in the mushy state. The dendrite arms at the bottom of the domain have more lateral growth than the arms at the top because of the temperature gradient.

Figure-5.13 show the duplex microstructure of RUN521. The core of the dendrite is composed of a ferrite phase, because the nominal alloy composition solidifies into a ferritic solid. However, as the temperature decreases due to the cooling, an austenite phase starts 
to grow around the grown ferrite. Therefore, the cooling rate is important in determining the fraction of ferrite and austenite in a duplex microstructure solidification. The produced fraction of austenite would increase if the cooling rate increases. The fraction of ferrite is equal to 0.67 and the fraction of austenite is equal to 0.33 . The simulation time was equal to $0.0124 \mathrm{sec}$, and it is expected that the fraction of austenite will increases if the simulation continues.
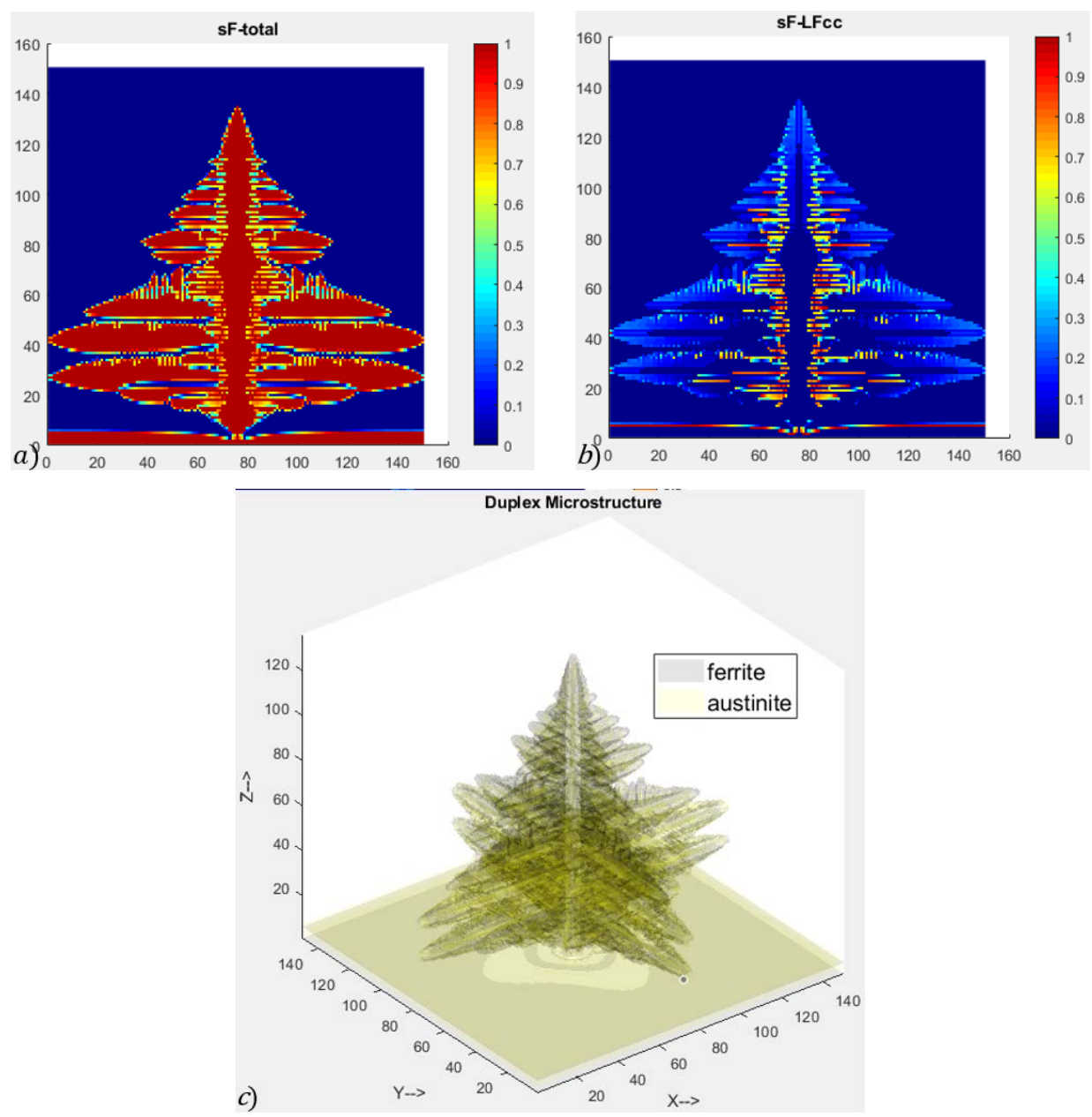

Figure-5.13: (a) the produced ferrite phase, (b) the produced austenite phase, and (c) the developed duplex microstructure. [RUN521] 
The same investigation of microstructure can be repeated for any point at the weld pool boundary. The results would be affected by the cooling rate and the temperature gradient that are vary according to the used heat input and the weld joint design. Decreasing the heat input (i.e. increase the welding speed or decrease the welding power) would increase the cooling rate and temperature gradient. Therefore, the developed dendrite would be more dendritic and fractal, the solute segregation would increase, and the microstructure would be more prone to weld decay. Increasing the thickness of the weld joint would result in the same effect. On the other hand, increasing the heat input would decrease the cooling rate and temperature gradient which has the opposite effect. 


\section{Chapter-6: Conclusions}

A CA-CALPHAD model was successfully developed by coupling the CALPHAD method with the cellular automata (CA) microstructure solidification model. The model assumes that the interfacial cells are at a thermodynamic equilibrium and assigns the equilibrium information to the interfacial cells during the simulation. Since the $\mathrm{S} / \mathrm{L}$ interface is at equilibrium, the values of phase fractions and solute concentrations in phases at a given temperature and an average composition in the cell are calculated by CALPHAD and assigned to the $\mathrm{S} / \mathrm{L}$ interfacial cells. Mathematically, the discontinuity between the liquid domain and the solid domain is treated as Dirichlet's boundary conditions to which the equilibrium information is assigned. The CA-CALPHAD model overcomes the limitation of the linearization approach used in other CA models available in the literature, in which the liquidus and solidus curves are considered as straight lines with constant slopes. Therefore, a wide range of practical alloys such as stainless steels can be investigated. Another advantage of the model is the possibility to model solidification producing different solid phases in the same alloy at different temperatures. The model validity is confirmed by comparing the results with the published CA model results and with the experimentally observed dendritic growth features presented in the literature.

In order to couple CALPHAD calculations with the CA model, an efficient lookup table with tie lines interpolation scheme was developed. The thermodynamic information was 
pre-calculated and stored in the table so that the required information was interpolated during the simulation. The table stores the information of the solidus and liquidus curves for many temperature values. Therefore, the required information including the phase fractions and the concentration of solutes in each phase can be interpolated. The size of the stored data required for the simulation of solidification in a $\mathrm{Fe}-\mathrm{Cr}-\mathrm{C}$ alloy is an order of one $\mathrm{MB}$ and the interpolation for a single point equilibrium is 300 times faster than the CALPHAD calculation.

The capillarity effect was taken into account using two different approaches: the cell-count method and the level-set method. The results demonstrate that each method predicts a different dendrite morphology, solute segregation, and dendrite growth rate. A comparison study for the two method was conducted, and it was found that the cell-count method is less accurate and has a higher numerical noise that can disturb a flat $\mathrm{S} / \mathrm{L}$ interface having just one nucleus at the center. As a result, the dendrite morphology and the solute segregation are affected significantly. In contrast, the level-set method is more accurate and has a lower numerical noise, and its accuracy increases as the mesh size decreases. Moreover, the level-set method is less sensitive to the mesh anisotropy. The accuracy of the level-set method was improved by averaging the solid fraction field with optimized weights.

The simulations of a free and constrained dendritic growth demonstrated that the segregation of chromium was less pronounced than the segregation of carbon, since the 
distribution coefficient of chromium was bigger. In addition, the solutes segregated at the boundary of the evolving dendrite more than at its core, and the solute segregation increases as the cooling rate increased. The simulations predicted that increasing the cooling rate produced more fractal dendrite (with more secondary and tertiary arms) and a finer grain size. Similarly, increasing the initial undercooling increased the segregation and the growth rate and produced a finer grain size.

The solidification of a duplex steel was investigated by using a nominal alloy composition, which was composed of both the ferrite and austenite phases. It was found that the austenite phase was formed around the ferrite phase, and more austenite phase fraction was obtained by increasing the cooling rate. This occurred because, as the temperature was lowered due to a higher cooling rate, a larger austenite fraction was produced.

A competitive growth of dendrites was investigated by initializing a rough initial $\mathrm{S} / \mathrm{L}$ interface. Initially, many dendrites started to grow and, eventually, few of them survived due to the competitive growth. This suggests that a steady growth state was established after some time of the simulation regardless of the initial state configuration and solute distribution.

In order to investigate the microstructure of the weld, the model was successfully coupled with $\operatorname{VrWeld}{ }^{\circledR}$, a computational welding mechanics software. The microstructure within the weld pool varies because of the variation in the thermal condition. At the rear-most point of the weld pool on the fusion line the cooling rate is maximum and the temperature 
gradient is minimum. Correspondingly, the solute segregation and the $\mathrm{C} / \mathrm{Cr}$ ratio are maximum, and the microstructure is finer. Therefore, the locations with high values of the cooling rate are more prone to the weld decay, since high $\mathrm{C} / \mathrm{Cr}$ ratios are predicted there. Finally, with a constant arc power, increasing the welding speed or increasing the weld joint transverse thickness will increase the cooling rate and affect the microstructure.

\section{Contribution to Knowledge}

- Coupling CALPHAD method with a cellular automata microstructure solidification modeling was successfully accomplished, and the limitation of the phase diagram linearization was overcome.

- An efficient data structure and interpolation scheme to store and interpolate the thermodynamic information were developed.

- The performances of the cell-count and level-set methods were compared, and the level-set method with an optimized accuracy was developed.

- The developed coupled model CA-CALPHAD can be used to investigate stainless steel alloys because the linearized approach to phase diagrams is overcome.

- The effects of cooling rates and temperature gradients on the dendrite size and morphology as well as the segregation pattern were predicted.

- The microstructure variations around the weld pool boundary was investigated for a practical stainless steel weld joint in response to realistic welding parameters.

- The microscopic solidification of a duplex steel and the potential of the weld decay were investigated for a practical weld joint and welding parameters. 


\section{Future Work}

1) Adding a nucleation algorithm based on CALPHAD to the model. A nucleation density function can be assumed, and the feasibility of nucleation can be determined by thermodynamics. In addition, the type of the nucleated phase (ferrite, austenite) can be determined by thermodynamics. Such algorithm is useful in studying the columnar-toequiaxed transition.

2) Modifying the anisotropy function and the capturing rule to allow for grain growth orientations. This addition produces grain with arbitrary orientation which is useful when nucleation algorithm is used.

3) Developing an implicit solver to solve the heat diffusion equation in the computational computational domain. In this case, the same time step can be used for the heat diffusion and the mass diffusion equations.

4) Developing more efficient and general interpolation scheme for thermodynamics information. 


\section{List of References}

[1] A. Artemev and J. Goldak, "Cellular Simulation of the Dendrite Growth in Al-Si Alloys", Canadian Metallurgical Quarterly, 36(1), 57-64, 1997.

[2] M. Martinez and A. Artemev, "Three Dimensional Computer Model of Dendrite Growth in Tertiary Al-Cu-Si Alloys", Master thesis of applied science, Canada: Carleton University, 1999.

[3] Ghazi Saati and A. Artemev, "Cellular Model Simulations of Solidification Structures in Ternary Alloys”, Master thesis of applied science, Canada: Carleton University, 2013.

[4] K. Komeil, J. Goldak and A. Artemev, "A Holistic Micro-Macro Model of an Arc Weld Pool with Microstructure Evolution in the Fusion and Heat-Affected Zones", Doctor of philosophy dissertation, Canada: Carleton University, 2014.

[5] J. Goldak et al, "How Can Computational Weld Mechanics Help Industry?", Welding Journal, 40-45, 2010.

[6] J. Goldak et al, "Why power per unit length of weld does not characterize a weld?", Computational Materials Science, 48, 390-401, 2010.

[7] R. Blondeau, Metallurgy and Mechanics of Welding Processes and Industrial Applications. $1^{\text {st }}$ John Wiley \& Sons Inc, 2008.

[8] J.C.M. Li, Microstructure and Properties of Materials. vol. 2, World Scientific, 2000.

[9] S. Kou, Welding Metallurgy. $2^{\text {nd }}$, John Wiley \& Sons Inc, 2003.

[10] J. Lippold, Welding Metallurgy and Weldability. $1^{\text {st }}$, John Wiley \& Sons Inc, 2015.

[11] J. Dantzig and M. Rappaz, Solidification. $1^{\text {st }}$, CRC Press, 2009.

[12] D. Stefanescu, Science and Engineering of Casting Solidification. $2^{\text {nd }}$, Springer, 2009.

[13] Chun-Pyo Hong, Computer Modelling of Heat and Fluid Flow in Materials. IOP Publishing Ltd, 2004.

[14] W. Kurz and D. Fisher, Fundamentals of Solidification. $4^{\text {th }}$, CRC Press, 1998.

[15] W. Kurz et al, "Progress in modelling solidification microstructures in metals and alloys: dendrites and cells from 1700 to 2000", International Materials Reviews, vol. 64, 311-354, 2019.

[16] J.S. Langer and H. Muller-Krumbhaar, "Stability Effects in Dendritic Crystal Growth", Journal of Crystal Growth, vol. 42, 11-14, 1977. 
[17] J. Lipton, M. Glicksman and W. Kurz, "Dendritic Growth into Undercooled Alloy Melts", Materials Science and Engineering, vol. 65. 57-63, 1984.

[18] M. Glicksman, Principles of Solidification: An Introduction to Modern Casting and Crystal Growth Concepts. Springer, 2010.

[19] Perez-Raya and S.G. Kandlikar, "Stefan Problem", Advances in Heat Transfer, 2016.

[20] T. Carozzani, Ch-A Gandin and H Digonnet, "Optimized parallel computing for cellular automaton-finite element modeling of solidification grain structures", Modelling Simul. Mater. Sci. Eng, vol 22, 2014.

[21] G. Schmitz and U. Prahl, Handbook of Software Solutions for ICME. Wiley-VCH Verlag GmbH \& Co, 2017.

[22] M. Rappaz and Ch.-A. Gandin, "Probabilistic Modelling of Microstructure Formation in Solidification Processes”, Aeta metall mater, vol. 41, 345-360, 1993.

[23] K. Reuther and M. Rettenmayr, "Perspectives for cellular automata for the simulation of dendritic solidification - A review", Computational Materials Science, 95, 213-220, 2014.

[24] G. Caginalp, "Stefan and Hele-Shaw type models as asymptotic limits of the phase-field equations", Physical Review A, 39 (11), 5887, 1989.

[25] A. Karma and W. Rappel, "Phase-Field Method for Computationally Efficient Modeling of Solidification with Arbitrary Interface Kinetics”, Physical Review, 53(4), 53-57, 1995.

[26] R. Kobayashi, "Modeling and numerical simulations of dendritic crystal growth", Physica D: Nonlinear Phenomena, 63(3), 410-423, 1993.

[27] M. Zaeem et al, "Modeling dendritic solidification of $\mathrm{Al}-3 \% \mathrm{Cu}$ using cellular automaton and phase-field methods", Applied Mathematical Modelling, 37, 3495-3503, 2013.

[28] A. Choudhury et al, "Comparison of phase-field and cellular automaton models for dendritic solidification in Al-Cu alloy", Computational Materials Science 55, 263-268, 2012.

[29] L. Nastac, Modeling and Simulation of Microstructure Evolution in Solidifying Alloys. Kluwer Academic Publishers, 2004.

[30] ASM international staff, (1992). Alloy Phase Diagrams. $3^{\text {rd }}$, ASM handbook, 1992.

[31] R. DeHoff, Thermodynamics in Materials Science. $2^{\text {nd }}$, Taylor \& Francis Group LLC, 2006.

[32] T. Nishizawa, Thermodynamics of Microstructures. ASM Intl, 2008.

[33] Y. Zuo and Y. A. Chang, "Thermodynamic Calculation of The Ai-Mg Phase Diagram", CALPHAD, vVol. 17, No. 2, 161-174, 1993.

[34] M. Hillert, "Partial Gibbs Energies from Redlich-Kister Polynomials", Thermochimica Acta, 129, 71-75, 1988. 
[35] D. Porter et al. Phase Transformations in Metals and Alloys. $3^{\text {rd }}$, Taylor \& Francis Group LLC, 2009.

[36] N. Saunders and A. Miodownik, CALPHAD (Calculation of Phase Diagrams): A Comprehensive Guide. Elsevier Science Ltd, 1998.

[37] U. R. Kattner, "The Thermodynamic Modeling of Multicomponent Phase Equilibria”, JOM, 1997.

[38] K. Janssens et al, Computational Materials Engineering an Introduction to Microstructure Evolution. Elsevier Inc, 2007.

[39] H. Lukas. Computational Thermodynamics: The Calphad Method. Cambridge university press, 2007.

[40] J. Andersson, "A Thermodynamic Evaluation of the Fe-Cr-C System", Metallurgical Transactions, 19A, 627-636, 1988.

[41] Alexandra Khvan et al, "A thermodynamic evaluation of the Fe-Cr-C system", CALPHAD 46, 24-33, 2014.

[42] MATLAB documentation - fsolve. Retrieved from https://www.mathworks.com/help/optim/ug/fsolve.html

[43] R. Lesar, Introduction to Computational Materials Science Fundamentals to Applications. $1^{\text {st }}$, Cambridge university press, 2013.

[44] D. Raabe et al, Continuum Scale Simulation of Engineering Materials Fundamentals Microstructures - Process Applications. 1 ${ }^{\text {st }}$, WILEY-VCH Verlag Gmbh \& Cokgaa, 2004.

[45] A. Z. Lorbiecka and B. Sarler, "Simulation of Dendritic Growth with Different Orientation by Using the Point Automata Method", CMC, vol.18, no.1, 69-103, 2010.

[46] Lei Wei et al, "Orientation select ion of equiaxed dendritic growth by three -dimensional cellular automaton model", Physica B 407, 2471-2475, 2012.

[47] M. Pietrzyk, Computational Materials Engineering. Elsevier Inc, 2015.

[48] S. Pan and M. Zhu, "A three-dimensional sharp interface model for the quantitative simulation of solutal dendritic growth", Acta Materialia 58, 340-352, 2010.

[49] L. Beltran-Sanchez and D. M. Stefanescu, "Growth of solutal dendrites - A cellular automaton model”, Int. J. Cast Metals 15, 251-256, 2002.

[50] Ch.-A. Gandin and M. Rappaz, “A 3D Cellular Automaton Algorithm for The Prediction of Dendritic Grain Growth”, Acta mater, 45, 2187-2195, 1997.

[51] W. Wang et al, "A model of solidification microstructures in nickel-based superalloys: predicting primary dendrite spacing selection”, Acta Materialia 51, 2971-2987, 2003. 
[52] L. Nastac, "A 3D stochastic mesoscopic model for prediction of microstructure evolution during solidification of dendritic alloys", Metall. Res. Technol. 111, 311-319, 2014.

[53] X. H. Zhan, "Dendritic grain growth simulation in weld molten pool based on CA-FD model”, Cryst. Res. Technol. 43, 253-259, 2008.

[54] Rihong Han, "Macro-micro modeling and simulation for the morphological evolution of the solidification structures in the entire weld", International Journal of Heat and Mass Transfer 106, 1345-1355, 2017.

[55] Ch.-A. Gandin and M. Rappaz, "A Coupled Finite Element-Cellular Automaton Model for The Prediction of Dendritic Grain Structures in Solidification Processes", Acta. mater. vol. 42, 2233-2246, 1994.

[56] Ch.-A. Gandin and M. Rappaz, “A 3D Cellular Automaton Algorithm for the Prediction of Dendritic Grain Growth”, Acta. mater., vol. 45, 2187-2195, 1997.

[57] Ch.-A. Gandin et al, "A Three-Dimensional Cellular Automaton-Finite Element Model for the Prediction of Solidification Grain Structures", Metallurgical and Materials Transactions $A$, vol. 30a, 3153, 1999.

[58] R. Sasikumar and R. Sreenivasan, "Two Dimensional Simulation of Dendrite Morphology", Acta Metallurgica et Materialia, 42(7), 2381-2386, 1994.

[59] R. Sasikumar and E. Jacob, "Simulation of Side Branch Evolution in Thermal Dendritic Grains”, Scripta Materialia, vol. 35, 505-510, 1996.

[60] V. Pavlyk and U. Dilthey, "Application of Cellular Automata Technique for Simulating Dendrite Morphology and Grain Growth", Trends in welding research: proceedings of the 5th International Conference, Pine Mountain, Georgia, USA, 1998.

[61] L. Nastac, "Numerical Modeling of Solidification Morphologies and Segregation Patterns in Cast Dendritic Alloys", Acta Mater, 47(17), 4253-4262, 1999.

[62] Rui Chen, “A Modified Cellular Automaton Model for the Quantitative Prediction of Equiaxed and Columnar Dendritic Growth", J. Mater. Sci. Technol., 30(12), 1311-1320, 2014.

[63] Hong Bao and Tian Hang, "The Bezier Curve Representation of Binary Phase Diagram", International Conference on Software Engineering and Computer Science, 2013.

[64] S.A. David, S.S. Babu, and J.M. Vitek, "Welding: Solidification and Microstructure", JOM, 2003.

[65] M. Zhu et al, "Numerical Simulation of Microstructure Evolution During Alloy Solidification by Using Cellular Automaton Method", ISIJ International, vol. 50, 1851-1858, 2010 .

[66] M.F. Zhu and D.M. Stefanescu, "Virtual front tracking model for the quantitative modeling of dendritic growth in solidification of alloys", Acta Materialia 55, 1741-1755, 2007. 
[67] W.Wang et al, "Development of a CA-FVM Model with Weakened Mesh Anisotropy and Application to Fe-C Alloy", Crystals, 2016.

[68] J. Lippold and J. Kotecki, Welding Metallurgy and Weldability of Stainless Steels. John Wiley \& Sons, Inc. 2005.

[69] VrWeld ${ }^{\circledR}$. Retrieved from http://www.goldaktec.com/vrweld.html\#

[70] J. Goldak and M. Asadi, "Challenges in Verification of CWM Software to Compute Residual Stress and Distortion in Welds", PVP, 2010.

[71] J. Goldak, "Distortion and Residual Stress in Welds: The Next Generation", Trends in Welding Research, Proceedings of the 8th International Conference, ASM, 2009.

[72] M. Coulombe, "Computational Fatigue Analysis of Welded Structures", Master thesis of applied science, Canada: Carleton University, 2008.

[73] P. Khoral, "Coupling Microstructure to Heat Transfer Computation in Weld Analysis", Master thesis of applied science, Canada: Carleton University, 1989.

[74] Weldability of Materials - Stainless Steel. Retrieved from https://www.twiglobal.com/technical-knowledge/job-knowledge/weldability-of-materials-stainless-steel-020.

[75] D. F. Almeida et al, "Numerical simulation of residual stresses induced by TIG butt-welding of thin plates made of AISI 316L stainless steel", Procedia Structural Integrity, vol. 5, 633$639,2017$.

[76] J. Goldak et al, "A new finite element model for welding heat sources", Metallurgical Transactions B, vol. 15, 299-305, 1984.

[77] S.G.R Brown and N.B. Bruce, "A 3-Dimensional Cellular Automaton Model of Free Dendritic Growth”, Scripta Metallurgica et Materialia, 32(2), 241-246, 1995.

[78] L. Beltran-sanchez and D.M. Stefanescu, "Growth of Solutal Dendrites - A Cellular Automaton Model”, International Journal of Cast Metals Research, 15(3), 251-256, 2002.

[79] S.G.R. Brown, T. Williams and J.A. Spittle, "Acellular Automaton Model of the Steady-State "Free" Growth of A Non-Isothermal Dendrite", Acta metall mater, 42(8), 2893-2898, 1994.

[80] E. Pereloma and D. Edmonds. Phase transformations in steels: Fundamentals and diffusioncontrolled transformations. vol 1, Woodhead Publishing Limited, 2012.

[81] D. Gaskell and D. Laughlin, Introduction to the Thermodynamics of Materials. $6^{\text {th }}$, CRC Press, 2018.

[82] M. Hillert, Phase Equilibria, Phase Diagrams and Phase Transformations Their Thermodynamic Basis. $2^{\text {nd }}$, Cambridge University Press, 2018.

[83] Zi-Kui Liu and Yi Wang, Computational Thermodynamics of Materials. Cambridge University Press, 2016. 
[84] M. Moran et al, Fundamentals of Engineering Thermodynamics. $7^{\text {th }}$, John Wiley \& Sons, Inc. 2011.

[85] F. Campbell, Elements of Metallurgy and Engineering Alloys. Asm Intl, 2008.

[86] F. Campbell, Phase Diagrams Understanding the Basics. ASM International, 2012.

[87] J. Davis, Alloying Understanding the Basics. ASM International, 2001.

[88] D. West, Ternary Equilibrium Diagrams. Chapman and Hall, 1982.

[89] W. Callister and D. Rethwisch, Materials Science and Engineering: An Introduction. $8^{\text {th }}$, John Wiley \& Sons, Inc. 2010.

[90] H. Fredriksson and U. Akerlind, Solidification and Crystallization Processing in Metals and Alloys. John Wiley \& Sons, Ltd. 2012.

[91] F. Campbell, Phase Diagrams Understanding the Basics. $1^{\text {st }}$, ASM International, 2012.

[92] R. Reed, J. Goldak and E.D. Hughes, "Phase Diagrams as a Minimization Problem", unpublished.

[93] W. Oberkampf, Verification and Validation in Scientific Computing. $1^{\text {st }}$, Cambridge University Press, 2010.

[94] J. Goldak and M. Akhlaghi, Computational Welding Mechanics. $1^{\text {st }}$, Springer, 2006.

[95] P.J. Spencer, “A brief history of CALPHAD”, Computer Coupling of Phase Diagrams and Thermochemistry, 32, 1-8, 2008.

[96] K.C. Kumar and P. Wollants, "Some guidelines for thermodynamic optimisation of phase diagrams", Journal of Alloys and Compounds, 320, 189-198, 2001.

[97] Y. S. Teh And G. P. Rangaiah, “A Study Of Equation-Solving And Gibbs Free Energy Minimization Methods For Phase Equilibrium Calculations”, Trans IChemE, vol 80, Part A, 2002.

[98] G. Cacciamani, “An Introduction to The Calphad Method and the Compound Energy Formalism (CEF)", Tecnol. Metal. Mater. Miner., vol 13, 16-24, 2016.

[99] Xiaobo Li and Ping Zhang, "Calculation of Phase Diagram with MATLAB”, IEEE, 2010.

[100] John Agren, “Calculation of phase diagrams: Calphad”, Metals and alloys, 1996.

[101] B. Reiser, “On the Lattice Stability of Metals”, Theoret. Chim. Acta 43, 37-43,1976.

[102] A. T. Dinsdale, "SGTE Data for Pure Elements", CALPHAD, vol. 15, 317-425, 1991.

[103] Bo Sundman, "The OpenCalphad thermodynamic software interface", Computational Materials Science 125, 188-196, 2016. 
[104] D. V. Nichita, "Multiphase equilibria calculation by direct minimization of Gibbs free energy with a global optimization method", Computers and Chemical Engineering 26, 1703-1724, 2002.

[105] W. Oldfield, "Computer Model Studies of Dendritic Growth", Materials Science and Engineering, 211-218, 1973.

[106] C.R. Swaminath and V.R. Voller, "A General Enthalpy Method for Modeling Solidification Processes”, Metallurgical transactions B, 1992.

[107] S. Kutluay et al, "The numerical solution of one-phase classical Stefan problem", Journal of Computational and Applied Mathematics 81, 135-144, 1997.

[108] M.E. Glicksman, “Capillary Phenomena During Solidification”, Journal of Crystal Growth 42, 347-356, 1977.

[109] J.E. Taylor, "II—-mean curvature and weighted mean curvature", Acta Metallurgica et Materialia, vol. 40, 1475-1485, 1992.

[110] K. R. Sultana et al, "Numerical techniques for solving solidification and melting phase change problems", Numerical Heat Transfer, Part B, vol. 73, 129-145, 2018.

[111] T. Haxhimali et al, "Orientation selection in dendritic evolution", Nature materials, vol. 5, 2006.

[112] D. W. Hahn and M. Ozisik, Heat Conduction. $3^{\text {rd }}$, John Wiley \& Sons, Inc., 2012.

[113] F. Kreith et al, Principles of Heat Transfer. $7^{\text {th }}$, Cengage Learning, 2012.

[114] M. Ozisik et al, Finite Difference Methods in Heat Transfer. $2^{\text {nd }}$, CRC Press, 2017.

[115] P. Majumdar, Computational Methods for heat and Mass Transfer. CRC Press, 2006.

[116] C. Song Wu, Welding Thermal Processes and Weld Pool Behaviors. CRC Press, 2010.

[117] D. Raabe, "Don't Trust your Simulation - Computational Materials Science on Its Way to Maturity?", Advanced Engineering Materials, 2002. 